December 2003 - NREL/TP-500-34755

\title{
Modeling of the UAE Wind Turbine for Refinement of FAST_AD
}

J.M. Jonkman

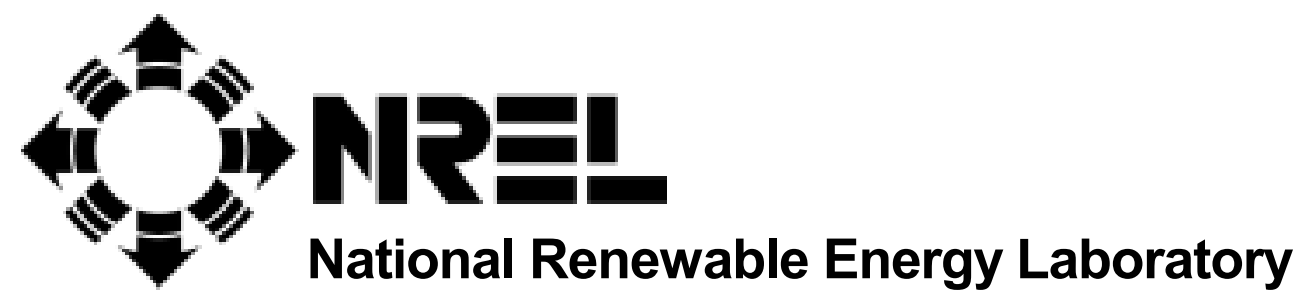

1617 Cole Boulevard

Golden, Colorado 80401-3393

NREL is a U.S. Department of Energy Laboratory Operated by Midwest Research Institute $\bullet$ Battelle

Contract No. DE-AC36-99-G010337 


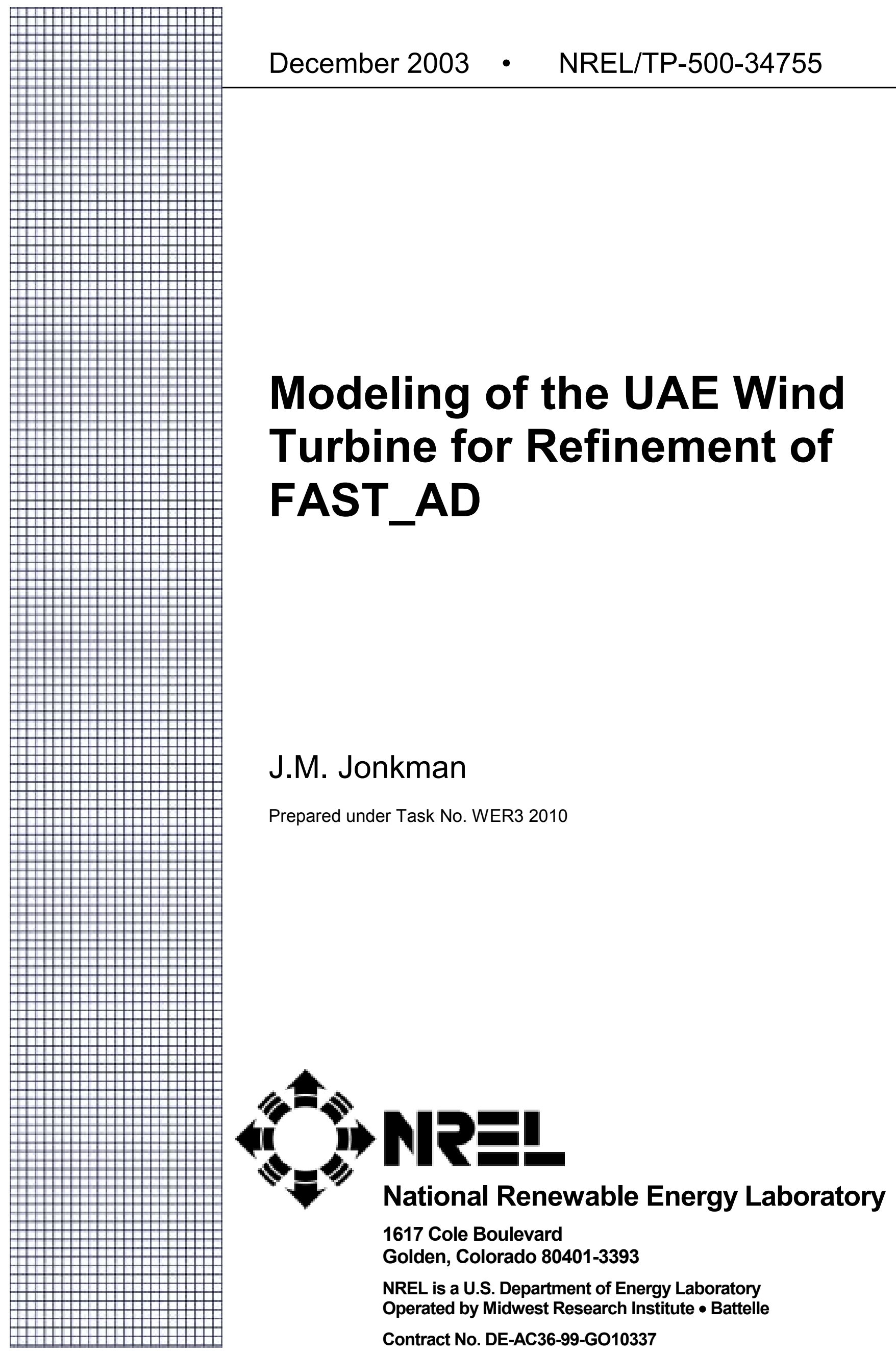




\section{NOTICE}

This report was prepared as an account of work sponsored by an agency of the United States government. Neither the United States government nor any agency thereof, nor any of their employees, makes any warranty, express or implied, or assumes any legal liability or responsibility for the accuracy, completeness, or usefulness of any information, apparatus, product, or process disclosed, or represents that its use would not infringe privately owned rights. Reference herein to any specific commercial product, process, or service by trade name, trademark, manufacturer, or otherwise does not necessarily constitute or imply its endorsement, recommendation, or favoring by the United States government or any agency thereof. The views and opinions of authors expressed herein do not necessarily state or reflect those of the United States government or any agency thereof.

Available electronically at http://www.osti.gov/bridge

Available for a processing fee to U.S. Department of Energy and its contractors, in paper, from:

U.S. Department of Energy

Office of Scientific and Technical Information

P.O. Box 62

Oak Ridge, TN 37831-0062

phone: 865.576 .8401

fax: 865.576.5728

email: reports@adonis.osti.gov

Available for sale to the public, in paper, from:

U.S. Department of Commerce

National Technical Information Service

5285 Port Royal Road

Springfield, VA 22161

phone: 800.553.6847

fax: 703.605.6900

email: orders@ntis.fedworld.gov

online ordering: http://www.ntis.gov/ordering.htm 


\begin{abstract}
The Unsteady Aerodynamics Experiment (UAE) research wind turbine was modeled both aerodynamically and structurally in the FAST_AD wind turbine design code, and its response to wind-inflows was simulated for a sample of test cases. A study was conducted to determine why there are wind turbine load magnitude discrepancies - inconsistencies in aerodynamic force coefficients, rotor shaft torque, and out-of-plane bending moments at the blade root across a range of operating conditions_- between load predictions made by FAST_AD and other modeling tools and measured loads taken from the actual UAE wind turbine during the NASAAmes wind tunnel tests. The NASA-Ames wind tunnel had a highly controlled testing

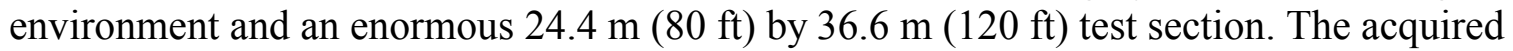
experimental test data represent the finest, most accurate set of wind turbine aerodynamic and induced flow field data available today. A sample of the FAST_AD model input parameters most critical to the aerodynamics computations was also systematically perturbed to determine their effect on load and performance predictions. Attention was focused on the simpler upwind rotor configuration, zero yaw error test cases. Inconsistencies in input file parameters, such as aerodynamic performance characteristics, explain a noteworthy fraction of the load prediction discrepancies of the various modeling tools. Additionally, discrepancies between modeling tool load predictions and physically measured load values highlighted weaknesses in tip loss, stall delay, post stall, and other frequently used aerodynamic models. Furthermore, unexplainable FAST_AD load prediction and measured load discrepancies uncovered flaws and limitations in the structural models employed by FAST_AD and suggested ways to improve models and codes. The ways these weaknesses and flaws affect the design of wind energy systems were noted. Additional development and refinement of rotary wing aerodynamics and structural models should lead to more optimal wind turbine designs and decreases in wind-generated electricity costs.
\end{abstract}




\section{Contents}

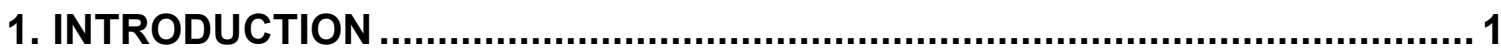

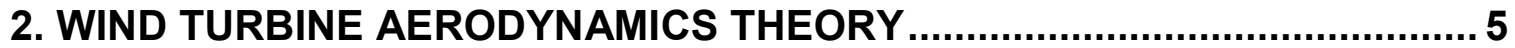

2.1 NATURAL WIND CHARACTERISTICS ................................................... 5

2.2 RANKINE-FROUDE ACTUATOR DISC THEORY AND BETZ LIMIT ......................... 6

2.3 EFFECTS OF WAKE ROTATION ON BETZ LIMIT ....................................... 10

2.4 BLADE ELEMENT THEORY ................................................................... 16

2.5 Blade Element Momentum Theory and Performance Prediction ......... 23

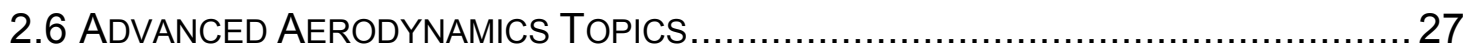

3. STRUCTURAL MODELING THEORY ….................................................. 29

3.1 GEOMETRY, CoORDINATE SYSTEMS, AND DEGREES OF FREEDOM .................29

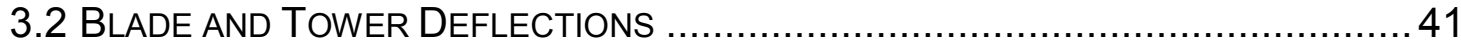

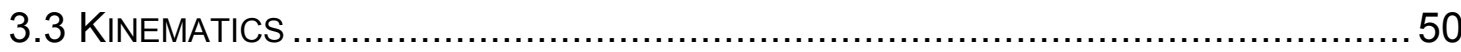

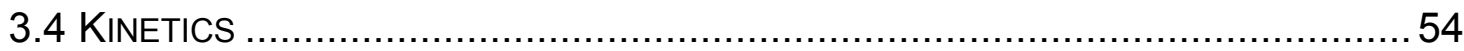

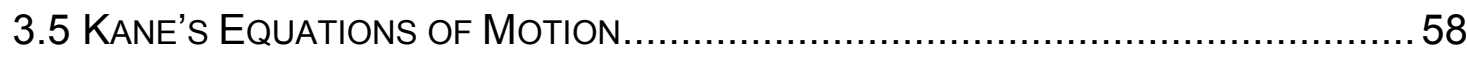

4. FAST_AD DESIGN CODE OVERVIEW AND LIMITATIONS ......................59

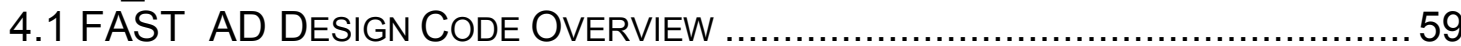

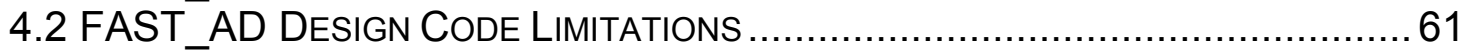

5. UAE AND BLIND COMPARISON OVERVIEW ........................................64

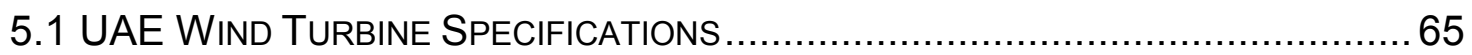

5.2 NASA-AMES WIND TUNNEL TESTING .................................................... 76

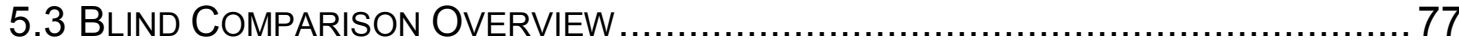

6. BLIND COMPARISON INPUT PARAMETERS AND RESULTS ....................81

6.1 FAST_AD INPUT FILES DEVELOPMENT FOR THE BLIND COMPARISON STUDY.. 81

6.2 BLIND COMPARISON RESULTS AND DISCUSSION .......................................... 98

7. SENSITIVITY STUDY INPUT PARAMETERS AND RESULTS ..................118

7.1 SENSITIVITY STUDIES AND FAST_AD INPUT PARAMETER ALTERATIONS .......118

7.2 SENSITIVITY STUDY RESULTS AND DISCUSSION .................................... 132

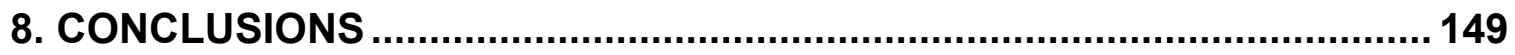

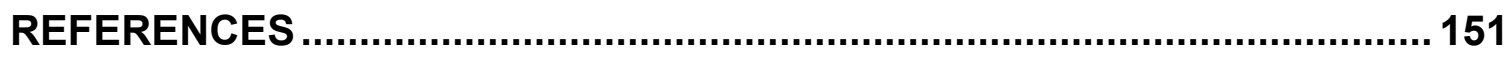

APPENDIX I. FAST_AD INPUT FILE FOR TEST CASE S0700000 ...............153

APPENDIX II. MODES INPUT FILES FOR USE IN FAST_AD ....................... 160

APPENDIX III. WIND DATA FILE FOR TEST CASE S0700000 …................. 162

APPENDIX IV. AIRFOIL DATA TABLE FILES OF THE S809 AIRFOIL..........163

APPENDIX V. CRUNCH DATA FILE FOR TEST CASE S0700000 ................173 


\section{Introduction}

Nonrenewable resources such as coal and natural gas are the main sources of energy for many parts of the world. However, these energy resources are harmful to the environment and supplies are limited. Resources such as wind possess great potential because they are renewable and nonpolluting.

Wind-driven power systems that can produce large amounts of power are relatively new technologies. Many earlier models failed catastrophically because blades struck their support structure or tore off the hubs, or towers collapsed. Such failures have diminished in recent years because of improved designs, but other failures such as unacceptable material fatigue and component malfunction are common. In general, failures occur because the effects of unanticipated loads and unknown load levels and load paths are substantial. To combat such effects, wind turbine designers rely heavily on time-tested safety factors that account for this lack of knowledge. Such a design methodology greatly limits the cost effectiveness of new wind turbine designs, which is critical if wind-generated electricity is to be economically competitive with traditional energy sources.

The U.S. Department of Energy's (DOE) National Renewable Energy Laboratory (NREL) has sponsored the development and validation of comprehensive system dynamics codes that can accurately predict, quantify, and understand the complex physical interactions that characterize component loads and overall wind turbine performance. Researchers and designers use such codes to efficiently, safely, and cost effectively design and analyze wind energy systems. These design codes enable the user to (1) model a wind turbine aerodynamically and structurally given the turbine layout and aerodynamic and mechanical properties of its members, and (2) simulate the wind turbine's aerodynamic and structural response by imposing complex virtual windinflow conditions. Outputs of these simulations include time-series data on the loads and responses of the structural members of the wind turbine. Post-processing codes are used to analyze these data.

In many respects, design codes (also labeled as modeling tools) bridge the gap between theorized predictions and experimental or observable measurements. Design codes essentially perform virtual experiments that can yield load analysis results quickly and cheaply. In many situations, virtual experimentation offers the only practical method of research and testing.

A streamlined design code titled FAST_AD was recently developed through a partnership between NREL and Oregon State University. FAST stands for Fatigue, Aerodynamics, Structures, and Turbulence modeling; the AD postscript indicates that the wind-inflow data used to excite the model during simulation routines are developed with the AeroDyn aerodynamics subroutine package, developed by engineering faculty and staff at the University of Utah.

Design codes must be validated to be effective in the design and analysis environment. Validation involves comparing simulated load predictions with load predictions made by other modeling tools and with measurements taken experimentally, then assessing the degree to which 
they correspond. FAST_AD has yet to be validated. For accurate and reliable experimental measurements to improve the model and validate the code, NREL planned and conducted the comprehensive test program known as the Unsteady Aerodynamics Experiment (UAE). In the most recent phase of this program (Phase VI), a heavily instrumented, 10-m diameter, 20-kW wind turbine, known as the UAE research wind turbine, was tested in the world's largest wind tunnel located at the NASA-Ames Research Center. These tests were completed in May 2000, following three weeks of exhaustive testing. The data acquired from the tests represent the finest, most accurate set of wind turbine aerodynamic and induced flow field data available.

To test the validity and range of applicability of the models used in design codes, NREL solicited wind turbine modeling experts from around the world to predict loads and performance of the UAE wind turbine at selected operating conditions. These predictions were done "blind" in that the only information given to the participants concerned basic machine parameters of the UAE wind turbine and essential wind-inflow data, not measured wind tunnel experimental data. During a later comparison phase, the predictions from all the Blind Comparison modelers were presented and compared with the experimentally measured data.

The objective of this report is to model the aerodynamic and structural aspects of the UAE research wind turbine in the FAST_AD design code and to run simulations by exciting the model with the same conditions seen in the NASA-Ames wind tunnel for several UAE test cases. Furthermore, to facilitate validation of FAST_AD, the load predictions are compared with load predictions made by similar modeling tools employed by other modelers during the Blind Comparison exercise and with experimental wind tunnel test measurements.

Load magnitude discrepancies, such as inconsistencies in aerodynamic force coefficients, rotor shaft torque, and out-of-plane bending moments at the blade root, appear between these various load predictions and measurements across a range of operating conditions. Some, but not all, are explainable by inconsistencies in the input file parameters of the modeling tools. Discrepancies among load predictions and measured load values highlight weaknesses in commonly used aerodynamic models and uncover flaws and limitations in the structural models employed by FAST_AD. Consequently, the aerodynamic and structural models engaged by FAST_AD should be refined before validation concludes. Moreover, the code should be progressively updated and revalidated as our understanding of the physics involved in wind turbine aerodynamics improves. Doing so will lead to more optimal wind turbine designs that are cheaper to manufacture and can produce cheaper wind-generated electricity.

Treating the FAST_AD design code as a "black box" would be careless if a reasonable study is to explain the discrepancies between code predictions and experimental test data, or even when simply assembling the FAST_AD input file. The assumptions and approximations used to develop the models employed by the code must be fully understood before any results can be characterized or any conclusions drawn. Thus, an exhaustive development of the aerodynamic and structural modeling theories of FAST_AD is presented here. Aerodynamic models are presented in Chapter 2 and structural models are presented in Chapter 3. During the development of these models, several flaws in the structural models implemented by FAST_AD are uncovered. These flaws are separate from any bugs in the coding. An exhaustive overview of the coding is not developed. The structural models derived in Chapter 3 are presented in their 
corrected form. Discrepancies between these models and those implemented in the FAST_AD design code are acknowledged using footnotes where appropriate. Code improvement recommendations for code designers of FAST_AD and the AeroDyn aerodynamics subroutines are italicized in these footnotes and in the main body of the report where appropriate.

An overview of the FAST_AD design code and descriptions of many of its limitations is given in Chapter 4. Descriptions of the UAE wind turbine, UAE NASA-Ames wind tunnel tests, and Blind Comparison exercise are presented in Chapter 5. A summary of the work performed to run simulations in FAST_AD for the Blind Comparison exercise and the associated results and discussion is given in Chapter 6. In this chapter, reasons for inconsistencies in the load predictions of the various modeling tools are explained, though an explanation of every load prediction discrepancy is not submitted. Additionally, weaknesses in the commonly used aerodynamic models are highlighted. Attention is focused on the descriptions of load prediction discrepancies and inaccuracies for the simpler upwind rotor configuration, zero yaw error test cases. To further account for and explain the load prediction and measured load discrepancies observed in the Blind Comparison results, a sensitivity analysis is performed as documented in Chapter 7. In the sensitivity study, a sample of FAST_AD model inputs is systematically perturbed to determine effects on load and performance predictions. A summary of conclusions is given in Chapter 8 .

To familiarize readers who are unfamiliar with wind turbine technology on the subject matter, various wind turbine classes and configurations are summarized, compared, and contrasted. Wind turbines harness the energy in the wind by extracting useful work. The power in the wind is extracted by a rotor, which consists of blades appended to a hub. The rotor extracts mechanical energy in the form of torque and rotational speed. Typically, this mechanical energy is converted to electrical energy by having the rotor run an electric generator, although the mechanical energy can also be used directly for applications such as pumping water.

All wind turbines can be characterized as either horizontal axis wind turbines (HAWTs) or vertical axis wind turbines (VAWTs). A HAWT is labeled as such since its rotor spins about an axis horizontal to the Earth's surface. The rotor of a VAWT spins about an axis perpendicular (vertical) to the Earth's surface. This report is devoted entirely to the analysis of HAWTs.

Rotors of HAWTs are placed on towers to position them where the wind speed is fastest and exhibits the most power. A nacelle typically resides atop the tower and contains the support structure for the rotor, the rotor shaft, a gearbox, and the electric generator. The gearbox is used to transform the low-speed, high-torque power of the rotor to high-speed, low-torque power that can run the electric generator.

Rotors can be positioned upwind or downwind of the tower. Downwind rotor configurations can track the wind automatically as wind directions change, similar to a weather vane (a passive yaw control system). However, the wind must flow around the tower to reach the rotor of a downwind turbine. This results in complex flow patterns and periodic fluctuations in aerodynamic loads, which have important dynamical effects on the turbine structure. Flow passing through the rotor plane is unobstructed by the tower for upwind rotor configurations. 
However, these turbines must possess an active yaw control system to keep the rotor aligned with the wind.

The hub structure connects the blades to the drive shaft. Hubs are generally characterized as either rigid or teetering. As the name implies, in rigid hub designs, the hub is rigidly attached to the drive shaft. In contrast, teetered hubs are connected to the drive shaft by means of a teeter pin, a bearing that permits the rotor to rock into and out of the plane of rotation. Teetered hubs have the benefit that bending moments brought about by thrust forces acting on the blades are not transferred to the nacelle and tower structure. Consequently, the nacelle and tower structures of turbines with rigid hubs must be designed more robustly than those with teetered hubs.

Rotor power is typically controlled by either pitch or stall regulation. Stall-regulated wind turbines take advantage of the fact that aerodynamic lift, and inevitably power, are reduced when the blades stall in exceptionally high winds. In pitch-controlled machines, power is regulated by varying the pitch of the blades (that is, the orientation of the blades about the blade axis) through the use of complex control mechanisms. In stall-regulated wind turbines, the blades of the rotor are rigidly connected to the hub. Though the hub design is simpler for stall-regulated machines, the drive train (rotor shaft, gearbox, and generator) must be more robustly designed than in pitchregulated machines so they can withstand extreme winds. These forces can be shed if a pitching mechanism is employed. 


\section{Wind Turbine Aerodynamics Theory}

Accurate models of the aerodynamic aspects of wind turbines are essential to successfully design and analyze wind energy systems. Wind turbine aerodynamic models are used to relate windinflow conditions to loads applied on the turbine. One must enter the field of wind turbine aerodynamics analysis with an appreciation for the complexity of the subject, however. After years of investigation and testing, researchers throughout Europe and the United States have concluded that the typical atmospheric conditions under which a wind turbine operates are incredibly complex. The underlying flow physics of wind turbine operation are also much more complex than originally thought.

The true fluid flow passing around and through a wind turbine is governed by the firstprinciples-based Navier-Stokes equations. Unfortunately, these equations are so complex that analytical solutions have been found for only a few simple cases. Although numerical solutions may be found via a computer, most first-principles-based solutions are computationally intense and the cost of such detailed simulations prohibits their use in the design and analysis environment. As an alternative, most wind turbine designers and analysts have opted to develop models that incorporate two-dimensional airfoil analysis techniques and quasi-steady flow fields.

The subsequent analysis develops the most common aerodynamics theory employed in the wind turbine design and analysis environment: blade element momentum theory (BEM), the fundamental aerodynamic model used by the AeroDyn aerodynamics subroutines of the FAST_AD design code. The final form of the BEM equations developed here, however, is not as common [see Eq. (2.73)], but enhances the visibility of the model's weaknesses. This attribute will become more apparent in the results and discussion of Chapter 6 . To help the reader appreciate the complexities of real fluid flow, we discuss natural wind characteristics qualitatively in section 2.1. Following a procedure similar to that used by McGowan and Manwell (2000), the BEM equations are developed in sections 2.2-2.5, with increasing complexity added in each section. A brief qualitative discussion of advanced aerodynamics topics is presented in section 2.6. This chapter and the aerodynamics theories developed are devoted entirely to HAWTs. Aerodynamic theories for VAWTs and other wind turbine configurations are beyond the scope of this report.

\subsection{Natural Wind Characteristics}

For practical reasons, wind turbines are positioned near ground level. As such, boundary layer effects with the Earth dominate airflow passing through wind turbines. Although the easiest way to regard the airflow is as steady, homogenous, uniform, and fixed in direction (see Fig. 2.1a), boundary layer flow is anything but. As a direct result of viscosity and the no-slip condition with the Earth's surface, boundary layer flow exhibits a velocity gradient with elevation. The simplest description of this tendency is the uniform wind shear model depicted in Fig. 2.1b. The boundary layer flow passing through a wind turbine is inherently inhomogeneous, unsteady, and turbulent, consisting of rapid velocity and pressure fluctuations. Turbulence occurs since inertial effects increasingly overwhelm viscous stresses inherent in the flow stream as the flow speed increases (even at very small wind speeds for air), resulting in intrinsically unstable flow that can become turbulent with even the slightest flow perturbation. Irregular terrain, such as mountains, 
valleys, houses, and trees increases the likelihood of turbulence at even the lowest wind speeds. Wind turbines with downwind rotors experience additional turbulence from the wake of the air passing around the tower and nacelle. Turbulent flow is depicted graphically in Fig. 2.1c.

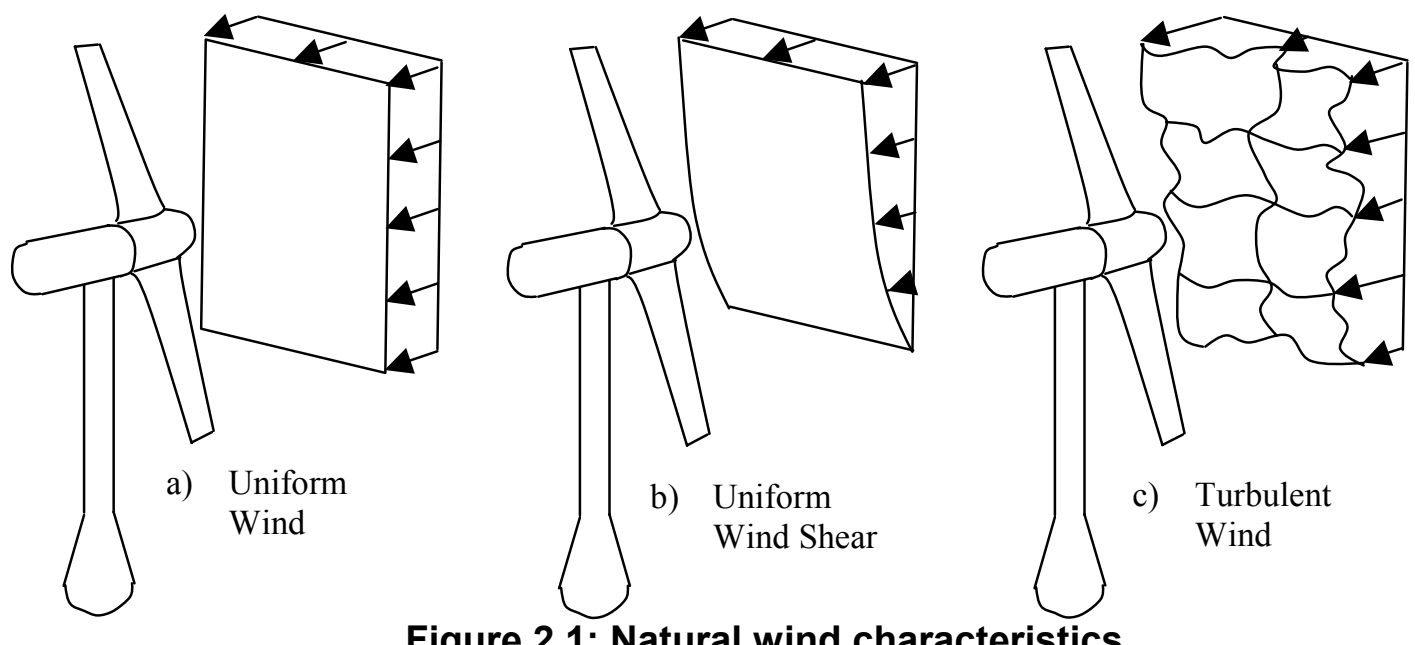

Figure 2.1: Natural wind characteristics

The situation is further complicated when wind directions change or the turbine becomes yawed. A rotor not aligned with the wind experiences cross-flow components whose magnitude and direction relative to the rotor change as the blades rotate. In such cases, flow separation and reattachment, spanwise flow, and three-dimensional effects become increasingly important. Moreover, the instability of the flow over the rotor blades is provoked by surface imperfections, squashed bugs, surface-water droplets, or ice buildup. Furthermore, blade vibration and torsional oscillation have important consequences to the form of the fluid flow. Vortices formed via flow interactions with the blade, hub, and tip also affect the character of the flow field in an integral way. Clearly, wind turbine aerodynamics is not an easy concept to grasp.

\subsection{Rankine-Froude Actuator Disc Theory and Betz Limit}

To begin to understand the complex physics involved in wind turbine aerodynamics, we should analyze a simple one-dimensional model. In the one-dimensional Rankine-Froude actuator disc model, the rotor is represented by an "actuator disc," through which the static pressure has a jump discontinuity. Consider a control volume fixed in space whose external boundaries are the surface of a stream tube whose fluid passes through the rotor disc, a cross-section of the stream tube upwind of the rotor, and a cross-section of the stream tube downwind of the rotor. A simple schematic of this control volume is given in Fig. 2.2.

The following assumptions are made in the actuator disc model:

(1) Wind is steady, homogenous, and fixed in direction.

(2) Air is incompressible, inviscid, and irrotational.

(3) Both the flow and the thrust are uniform across the disc. The flow is uniform at the upwind (station 0 ) and downwind (station 3) boundaries of the control volume. 


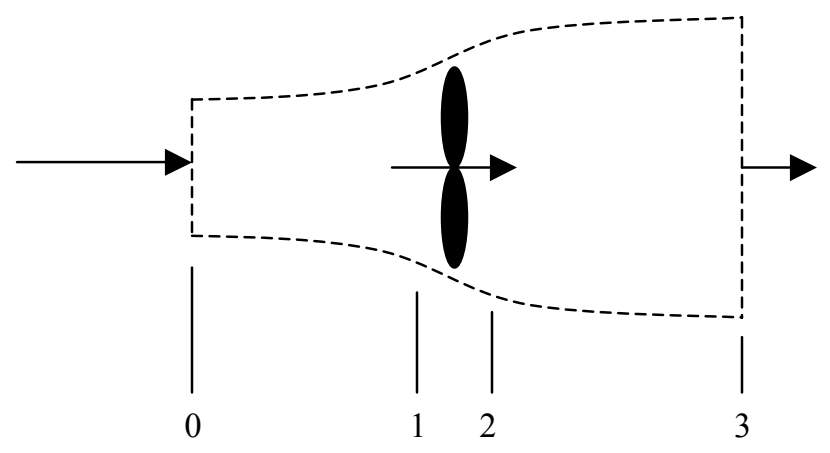

Figure 2.2: Control volume for actuator disc model

(4) The upwind and downwind boundaries are far enough removed from the rotor that the static pressure at these points is equal to the unobstructed ambient static pressure. The static pressure on the stream tube portion of the boundary is also equal to the unobstructed ambient static pressure.

For a wind turbine rotor to act as an actuator disc, the rotor would have to be composed of an infinite number of very thin, dragless blades that rotate with a tip speed much higher than that of the incoming wind. Also, station 1 is designated to be slightly upwind and station 2 slightly downwind of the rotor.

Since air does not pass through the stream tube portion of the control volume boundary by definition of a stream tube, applying the conservation of mass ${ }^{1}$ to the control volume yields:

$$
V_{0} A_{0}=V_{1} A_{1}=V_{2} A_{2}=V_{3} A_{3}
$$

where $V_{i}$ is the wind speed at station $i$ and $A_{i}$ is the cross section area of station $i$. Since $V_{l}$ is equal to $V_{2}$ according to assumption (3), and since $A_{1}$ equals $A_{2}$ by necessity, the components of the wind speed and cross sectional area at the plane of the disc are designated without subscripts for the remainder of this analysis (i.e., $V=V_{1}=V_{2}$ and $A=A_{1}=A_{2}$ ).

The thrust at the rotor disc, $T$, can be found by applying the conservation of linear momentum ${ }^{2}$ to the control volume in the axial direction. This process results in:

${ }^{1}$ The conservation of mass, which asserts that the instantaneous rate of change of mass within a control volume must equal the net flux of mass out of the control volume, can be stated as:

$$
\iiint_{V} \frac{\partial \rho}{\partial t} d V+\oiint_{S} \rho \boldsymbol{u} \cdot \boldsymbol{n} d S=0
$$

2 The conservation of linear momentum, which asserts that the instantaneous rate of change of linear momentum in the control volume plus the net flux of linear momentum out of the control volume must equal the sum of the net pressure forces and the net body force exerted by the surroundings on the control volume, can be stated as:

$$
\iiint_{V} \frac{\partial}{\partial t}(\rho \boldsymbol{u}) d V+\oiint_{S} \rho \boldsymbol{u}(\boldsymbol{u} \cdot \boldsymbol{n}) d S=-\oiint_{S} p \boldsymbol{n} d S+\iiint_{V} \rho \boldsymbol{f} d V
$$




$$
T=\rho A_{0} V_{0}^{2}-\rho A_{3} V_{3}^{2}
$$

or equivalently, using Eq. (2.1):

$$
T=\rho A V\left(V_{0}-V_{3}\right)
$$

where $\rho$ is the density of the air. The thrust at the rotor disc, $T$, is also the differential pressure between stations 1 and 2 multiplied by the disc area:

$$
T=\left(p_{1}-p_{2}\right) A
$$

where $p_{i}$ is the static pressure at station $i$. Since no work is done on either side of the turbine rotor, Bernoulli's equation ${ }^{3}$ can be applied to obtain the pressures incorporated into Eq. (2.4):

$$
\begin{gathered}
p_{0}+\frac{1}{2} \rho V_{0}^{2}=p_{1}+\frac{1}{2} \rho V^{2} \\
\text { and } \\
p_{3}+\frac{1}{2} \rho V_{3}^{2}=p_{2}+\frac{1}{2} \rho V^{2}
\end{gathered}
$$

where $p_{i}$ is again the static pressure at station $i$. Pressures $p_{0}$ and $p_{3}$ are identical by assumption (4), so pressures $p_{1}$ and $p_{2}$ can be eliminated from the thrust Eq. (2.4) with the help of Eqs. (2.5) and Eq. (2.6) to obtain:

$$
T=\frac{1}{2} \rho A\left(V_{0}^{2}-V_{3}^{2}\right)
$$

Eliminating the thrust at the rotor disc from Eqs. (2.3) and (2.7), the velocity of the flow through the rotor disc is the average of the upwind (free stream) and downwind velocities:

$$
V=\frac{V_{0}+V_{3}}{2}
$$

\footnotetext{
${ }^{3}$ Converting the conservation of linear momentum to differential form and considering steady, incompressible, and irrotational flows subject to conservative body forces only, one can derive Bernoulli's equation to be:

$$
p+\frac{1}{2} \rho \boldsymbol{u} \cdot \boldsymbol{u}+\rho v=\text { cons tan } t
$$

where the quantity $v$ is the body-force potential. For example, if gravity is considered to be the only conservative body force acting on the system, the body-force potential, $v$, would equal the product of the gravitational acceleration and the elevation relative to some datum.
} 
An axial induction (or interference) factor, $a$, is customarily defined as the fractional decrease in wind velocity between the free stream and the rotor plane:

$$
a=\frac{V_{0}-V}{V_{0}}
$$

or equivalently:

$$
\begin{array}{r}
V=V_{0}(1-a) \\
\text { and } \\
V_{3}=V_{0}(1-2 a)
\end{array}
$$

The velocity lost at the rotor plane, $V_{0}-V$, in Eq. (2.9) is known as the induced velocity.

As $a$ increases from zero, the downwind flow speed steadily decreases until, at $a=1 / 2$, it has completely stopped and the simple theory is no longer applicable.

Substituting for $V_{3}$ from Eq. (2.11), Eq. (2.7) can be rewritten in a more useful manner:

$$
T=\frac{1}{2} \rho A V_{0}^{2} 4 a(1-a)
$$

The power extracted from the wind by the rotor, $P$, is the product of the thrust, $T$, from Eq. (2.12) and the wind velocity at the rotor plane, $V$, from Eq. (2.10):

$$
P=\frac{1}{2} \rho A V_{0}^{3} 4 a(1-a)^{2}
$$

The commonly used nondimensional power coefficient, $C_{P}$, representing the fraction of available power in the wind that is extracted by the turbine, is defined as:

$$
C_{P}=\frac{P}{\frac{1}{2} \rho A V_{0}^{3}}
$$

Substituting the extracted power from Eq. (2.13) into Eq. (2.14) yields:

$$
C_{P}=4 a(1-a)^{2}
$$

The theoretical maximum power coefficient from an idealized rotor, $C_{P \max }$, known as Betz limit, can be found by setting the derivative of Eq. (2.15) with respect to $a$ equal to zero, and solving for $a$ : 


$$
\frac{\partial C_{P}}{\partial a}=4\left(1-3 a^{2}\right)=0 \ldots \text { yields } \ldots a=\frac{1}{3}
$$

Substituting this result into Eq. (2.15) yields:

$$
C_{P \max }=\frac{16}{27} \approx 0.59259
$$

The maximum possible efficiency for an idealized wind turbine is roughly $59.3 \%$. In practice, three effects prohibit a real wind turbine from achieving this efficiency:

(1) Rotation of the wake caused by the spinning rotor.

(2) Finite numbers of blades.

(3) Viscid flow causes nonzero aerodynamic drag.

All these effects will be considered in the sections that follow.

This one-dimensional model is simple and does not describe the true nature of the physical flow around wind turbines; however, it does bring to light several concepts that are key to understanding wind turbine operation. If the rotor is to extract any power from the wind, the wind must slow down as it passes through the rotor. An ideal wind turbine would have to slow the wind velocity at the rotor plane to two-thirds of the free stream value if it is to extract power at maximum efficiency. Thus, from continuity [Eq. (2.1)] the effective upstream area is less than the swept area of the rotor and the area of the wake downstream is greater than the swept area of the rotor. For an ideal wind turbine operating at maximum efficiency, the effective upstream area is two-thirds the swept area and the area of the wake downstream is twice the area swept by the rotor. Finally, extracting all the power available in the wind is theoretically impossible. In practice, a real wind turbine generally does not achieve a power coefficient of more than $45 \%$.

\subsection{Effects of Wake Rotation on Betz Limit}

In the previous model, assumption (1) stated that the rotor imparted no angular momentum to the wake. However, conserving angular momentum necessitates rotation of the wake if the rotor is to extract useful torque. Moreover, the flow behind the rotor will rotate in the opposite direction as the rotor in reaction to the flow imparting torque on the rotor as shown in Fig. 2.3:

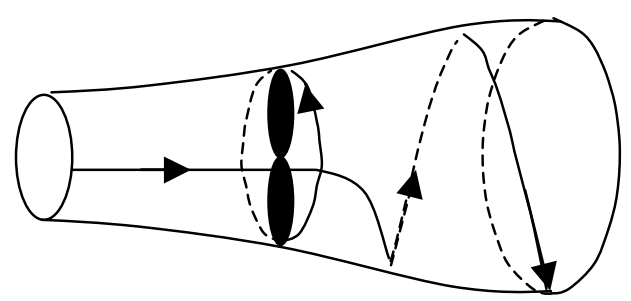

Figure 2.3: Wake rotation 
Since the wake is now rotating, it exhibits rotational kinetic energy that reduces the amount of available energy that can be extracted as useful work. As explained in the subsequent analysis, a rotor generating high torque at low speed will generate less power than a rotor generating low torque at high speed. The typical multibladed farm windmill is a notable example of a wind turbine whose efficiency is severely limited by wake rotation.

The Rankine-Froude actuator disc model can be easily extended to account for this wake rotation. To do this, assumptions (1) and (3) from the previous analysis can be relaxed and three supplementary assumptions added:

(1) The control volume used in the previous one-dimensional model can be split into many noninteracting annular stream tube control volumes.

(2) The flow entering the control volume(s) far upstream remains purely axial and uniform.

(3) The angular speed of the wake far downstream of the rotor is low so the static pressure far downstream can still be assumed to be identical to the unobstructed ambient static pressure.

Assumption (1) disregards assumption (3) from the previous analysis and allows for the local pressures, axial velocities (and induction factor), angular velocities, thrust, and power to all be functions of the annular radius. This point of view is illustrated in Fig. 2.4:

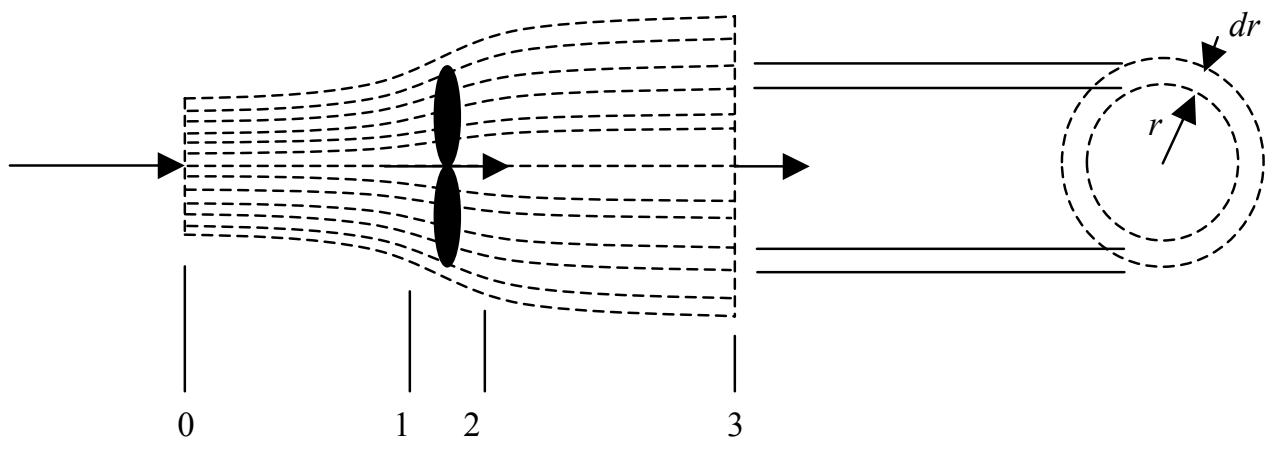

Figure 2.4: Annular stream tube control volumes

Assumption (3) in the analysis of the Rankine-Froude actuator disc model could have been relaxed in the analysis if the variables $A_{i}, T$, and $P$ were replaced with the differentials, $d A_{i}, d T$, and $d P$, respectively. In this case, the differential area of an annular ring at station $i, d A_{i}$, is defined as:

$$
d A_{i}=2 \pi r_{i} d r_{i}
$$

where $r_{i}$ and $d r_{i}$ are the radius and differential radius, respectively, of an annular ring at station $i$. To be precise, the radius and differential radius of the annular ring of Fig. 2.4 are shown as being the radius and differential radius, respectively, of an annular ring at station 3 , or $r_{3}$ and $d r_{3}$.

Although wake rotation is now included in the analysis, the assumption that the flow is irrotational [assumption (2) from the previous analysis] has not been lifted. The definition of 
rotational flow is any flow with nonzero vorticity. A fluid particle that moves in a circular trend but always retains its original orientation has zero vorticity and is considered to be irrotational. A classic example of this type of flow is a potential vortex.

Since applying the conservation of mass is not affected by wake rotation, the conservation of mass for an annular stream tube control volume can be found by substituting $d A_{i}$ from Eq. (2.18) in for $A_{i}$ in Eq. (2.1) to yield:

$$
V_{0} 2 \pi r_{0} d r_{0}=V 2 \pi r d r=V_{3} 2 \pi r_{3} d r_{3}
$$

where the components of velocity and annular area at the plane of the disc are designated without subscripts $\left(V=V_{1}=V_{2}, r=r_{1}=r_{2}\right.$, and $\left.d r=d r_{l}=d r_{2}\right)$. This designation is made throughout the remainder of the analysis.

The conservation of angular momentum ${ }^{4}$ about an axis consistent with the control volume's axis of symmetry can be applied to obtain the differential torque at the rotor disc, $d Q$, resulting in:

$$
d Q=\rho \omega_{3} r_{3}^{2} V_{3} 2 \pi r_{3} d r_{3}
$$

where $\omega_{3}$ is the angular velocity of the wake at station 3 defined so the differential rotor torque is positive. By assumption (1), the angular velocity of the flow stream at station $i, \omega_{i}$, is also a function of the annular radius.

Since the flow entering the control volume(s) far upstream is purely axial according to assumption (2), applying the conservation of angular momentum between stations 0 and 1 necessitates that there is no mechanism to impart rotational momentum to the stream ahead of the rotor. Thus,

$$
\omega_{0}=\omega_{1}=0
$$

Similarly, angular momentum is conserved between stations 2 and 3:

$$
\omega_{2} r^{2}=\omega_{3} r_{3}^{2}
$$

The angular velocity of the flow stream is discontinuous across the rotor plane because torque is exerted onto the rotor. Also, since the downwind radius of the control volume is larger than the radius of the control volume at the plane of the rotor, the wake rotation slows with distance from the rotor. This latter outcome correlates agreeably with assumption (3).

\footnotetext{
${ }^{4}$ The conservation of angular momentum, which asserts that the instantaneous change rate of angular momentum in the control volume plus the net flux of angular momentum out of the control volume must equal the sum of the net pressure moments and the net body moments exerted by the surroundings on the control volume, can be stated as:

$$
\iint_{V} \frac{\partial}{\partial t}(\rho \boldsymbol{r} \times \boldsymbol{u}) d V+\oiint_{S}(\rho \boldsymbol{r} \times \boldsymbol{u})(\boldsymbol{u} \cdot \boldsymbol{n}) d S=-\oiint_{S} \boldsymbol{p} \boldsymbol{r} \times \boldsymbol{n} d S+\iint_{V} \rho \boldsymbol{r} \times \boldsymbol{f} d V
$$
}


By combining Eqs. (2.20), (2.19), and (2.22), the differential rotor torque can be rewritten as:

$$
d Q=\rho \omega_{2} r^{2} V 2 \pi r d r
$$

In this form, as the torque of the rotor is increased, the angular speed of the wake increases accordingly.

Since applying the conservation of linear momentum in the axial direction is unaffected by wake rotation, the differential thrust at the rotor disc, $d T$, can be found by substituting $d A_{i}$ from Eq. (2.18) at station 1 (equivalently station 2) in for $A$ in Eq. (2.12) to yield:

$$
d T=\frac{1}{2} \rho 2 \pi r d r V_{0}^{2} 4 a(1-a)
$$

where the induction factor, $a$, is now a function of the annular radius.

The differential thrust at the rotor disc, $d T$, is also governed by Eq. (2.4) if $d A_{i}$ from Eq. (2.18) at station 1 (equivalently station 2 ) is again substituted for $A$. This substitution results in:

$$
d T=\left(p_{1}-p_{2}\right) 2 \pi r d r
$$

where the local pressures at stations 1 and $2, p_{1}$ and $p_{2}$ respectively, are now both functions of the annular radius.

To arrive at a description of the wake rotation, it is beneficial to find the difference in pressures $p_{1}$ and $p_{2}$ not by Bernoulli's equation, but ${ }^{5}$ :

$$
p_{1}+\frac{1}{2} \rho V^{2}=p_{2}+\frac{1}{2} \rho\left(V^{2}+\omega_{2}^{2} r^{2}\right)+\frac{d Q \Omega}{V 2 \pi r d r}
$$

The product of the differential rotor torque, $d Q$, and the angular speed of the rotor, $\Omega$, in Eq. (2.26) represents the differential power extracted by the turbine, $d P$.

Substituting Eq. (2.23) for $d Q$ in Eq. (2.26), solving for the difference in pressure, $p_{1}-p_{2}$, and substituting the result into Eq. (2.25) results in an alternative relation for the differential rotor thrust, $d T$ :

\footnotetext{
${ }^{5}$ The approximate form of the energy equation is a replacement for Bernoulli's equation when viscous, heat transfer, work insertion, and work extraction effects are important. The approximate form of the energy equation can be written as:$$
p_{1}+\frac{1}{2} \rho \alpha_{1} \bar{u}_{1}^{2}+\frac{\rho \dot{W}_{p}}{\dot{m}}=p_{2}+\frac{1}{2} \rho \alpha_{2} \bar{u}_{2}^{2}+\frac{\rho \dot{W}_{t}}{\dot{m}}+\rho g h_{L}
$$ 


$$
d T=\rho\left(\Omega+\frac{1}{2} \omega_{2}\right) \omega_{2} r^{2} 2 \pi r d r
$$

An angular induction factor, $a^{\prime}$, is customarily defined as:

$$
a^{\prime}=\frac{\omega_{2}}{2 \Omega}
$$

or equivalently:

$$
\begin{gathered}
\omega_{2}=2 \Omega a^{\prime} \\
\text { and } \\
\omega_{3}=2 \Omega a^{\prime}\left(\frac{r}{r_{3}}\right)^{2}
\end{gathered}
$$

by virtue of Eq. (2.22). The $1 / 2$ is used in the definition of the angular induction factor $a$ ' since the angular velocity of the flow stream at the plane of the rotor (at the jump discontinuity), $\omega$, is the average value of the angular velocity just upwind and downwind of the rotor:

$$
\omega=\frac{\omega_{1}+\omega_{2}}{2}
$$

or:

$$
\begin{aligned}
\omega & =\frac{\omega_{2}}{2} \\
a^{\prime} & =\frac{\omega}{\Omega} \\
\text { and } & \\
\omega & =\Omega a^{\prime}
\end{aligned}
$$

by virtue of Eqs. (2.21) and (2.28).

The differential rotor thrust, $d T$, and differential rotor torque, $d Q$, can now be written in terms of the two induction factors, $a$ and $a$ ':

$$
d T=\rho \Omega^{2} r^{2} 4 a^{\prime}\left(1+a^{\prime}\right) \pi r d r
$$


and

$$
d Q=\rho V_{0} \Omega r^{2} 4 a^{\prime}(1-a) \pi r d r
$$

Equating the differential rotor thrust from Eq. (2.35) with Eq. (2.24), the two induction factors are related:

$$
\frac{a(1-a)}{a^{\prime}\left(1+a^{\prime}\right)}=\left(\frac{\Omega r}{V_{0}}\right)^{2}
$$

The local speed ratio, $\lambda_{r}$, is defined as the square root of the right hand side of this equation:

$$
\lambda_{r}=\frac{\Omega r}{V_{0}}
$$

This variable is an expanded definition of the more commonly used tip speed ratio, defined as the dimensionless number corresponding to the blade tip speed, $\Omega R$, divided by the velocity of the free stream wind speed, $V_{0}$ :

$$
\lambda=\frac{\Omega R}{V_{0}}
$$

where $R$ is the radius of the turbine blades.

Substituting the local speed ratio, $\lambda_{r}$, from Eq. (2.38) into Eq. (2.37), and solving for $a$ ', the angular induction factor is written in terms of the axial induction factor and the local speed ratio:

$$
a^{\prime}=\frac{1}{2}\left(\sqrt{1+\frac{4}{\lambda_{r}^{2}} a(1-a)}-1\right)
$$

As $a$ increases from zero, the magnitude of $a^{\prime}$ (and thus the magnitude of the angular speed of the wake accordingly) also increases. The magnitude of this increase is limited by the local speed ratio. The faster the blade spins relative to a given free stream wind speed, the smaller the angular induction factor and angular speed of the wake.

Following a procedure similar to that used when finding Betz limit in section 2.2, the equations developed thus far can be used to modify the theoretical maximum power coefficient for an idealized rotor, $C_{P \max }$, accounting for wake rotation. The results of this analysis are presented graphically in Fig. 2.5: 


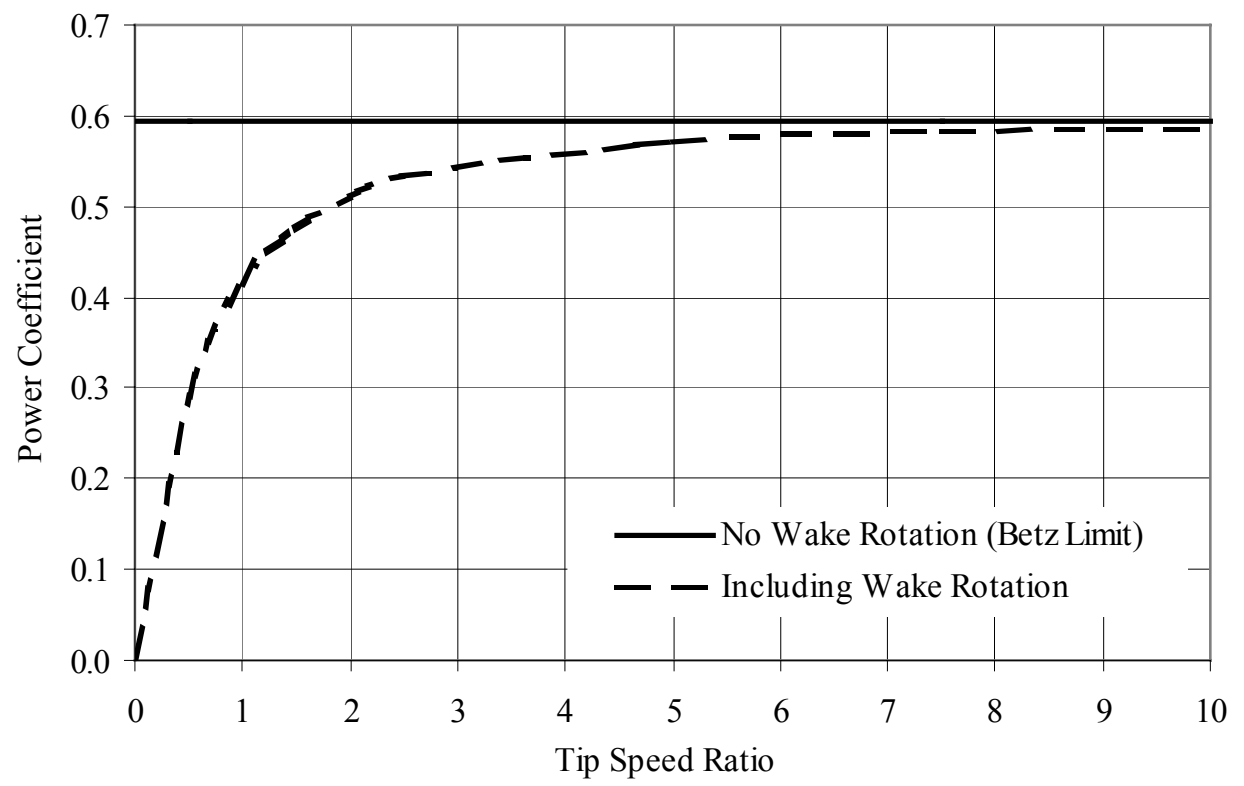

Figure 2.5: Theoretical maximum power coefficient

\subsection{Blade Element Theory}

In the previous two sections, forces exerted on a wind turbine rotor by the flow stream (thrust and torque) were found by applying principles of mass and momentum conservation on annular control volumes surrounding the flow. This type of analysis is commonly referred to as momentum theory. However, unless some flow state is assumed, there are not enough equations to solve for the differential rotor thrust, $d T$, and differential rotor torque, $d Q$, at a given span location on the rotor (a specified $r$ ) even if the air density, $\rho$, free stream wind speed, $V_{0}$, and angular speed of the rotor, $\Omega$, are known. That is, even though the angular induction factor, $a^{\prime}$, is related to the axial induction factor, $a$, by Eq. (2.40), the value of $a$ must be assumed at the span location in question, if the differential rotor thrust, $d T$, and differential rotor torque, $d Q$, are to be discerned. Thus, additional equations concerning the state of flow must be developed.

This state of flow is governed by the characteristics of the rotor blades, such as airfoil shape and twist distribution. The type of analysis that uses these blade geometry properties to determine the forces exerted on a wind turbine by the flow stream is referred to as blade element theory. As the word element in the theory title suggests, blade element theory again uses several annular stream tube control volumes. At the rotor plane, the boundaries of these control volumes effectively split the blade into a number of distinct elements, each of length $d r$. At each element, blade geometry and flow stream properties can be related to a differential rotor thrust, $d T$, and a differential rotor torque, $d Q$, if a number of assumptions are made. In blade element theory, the following assumptions are customarily made:

(1) Just as the annular stream tube control volumes used in the wake rotation analysis were assumed to be noninteracting [assumption (1)], it is assumed that there is no interaction between the analysis of each blade element.

(2) The forces exerted on the blade elements by the flow stream are determined solely by the two-dimensional lift and drag characteristics of the blade element airfoil shape and 
orientation relative to the incoming flow. The term two-dimensional implies that no threedimensional flow effects are assumed to occur around each blade element.

As discussed in Chapter 6, these assumptions are flawed and will bring about disagreements between modeled parameters and true physical parameters, including force coefficients, pressure distributions, and angle of attack distributions.

The forces exerted on an object by fluid as it flows over the object are due to pressure and viscous stresses. On the upper surface of an airfoil, a two-dimensional cross-section of a blade, the pressure is less than that of the incoming flow stream and effectively "sucks" the airfoil upward, normal to the incoming flow stream. By contrast, the pressure on the lower surface of the airfoil is greater than that of the incoming flow stream and effectively "pushes" the airfoil upward, normal to the incoming flow stream. The components of the pressure distribution parallel to the incoming flow stream tend to slow the velocity of the incoming flow relative to the airfoil, as do the viscous stresses. A depiction of the pressure and viscous stress distribution around a typical airfoil is given in Fig. 2.6:

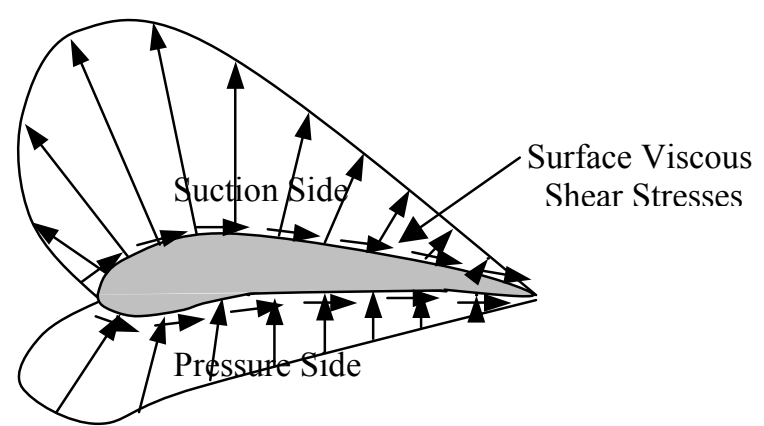

Figure 2.6: Pressure and viscous stresses exerted on an airfoil

On an airfoil, the resultants of these forces are usually resolved into two forces and one moment. The component of the net force acting normal to the incoming flow stream is known as the lift force and the component of the net force acting parallel to the incoming flow stream is known as the drag force. The effective moment, known as the pitching moment, is usually defined about an axis normal to the airfoil cross-section, located a quarter of the distance from the leading edge to the trailing edge of the airfoil. The pressure distribution on both sides of the airfoil contributes to the lift (see Fig. 2.6). The part of the drag force related to the pressure distribution around the airfoil is known as the pressure drag. The part of the drag force related to the viscous stresses is known as the skin-friction drag. Their sum, the total drag force, is commonly referred to as form drag. The viscous stresses generally make a negligible contribution to the lift force.

As an alternative to the lift and drag forces, analyses are commonly made with normal and tangential forces. The normal force is the component of the net force acting normal to the chord line and the tangential force is the component of the net force acting parallel to the chord line (the straight line that connects the leading and trailing edges of the airfoil). For a given flow condition, the relationships between the lift and drag forces and the normal and tangential forces are purely vectorial as determined by the angle of attack of the incoming flow stream. The angle 
of attack, $\alpha$, is defined as the angle between the incoming flow stream and the chord line of the airfoil. These characteristics are depicted in Fig. 2.7.

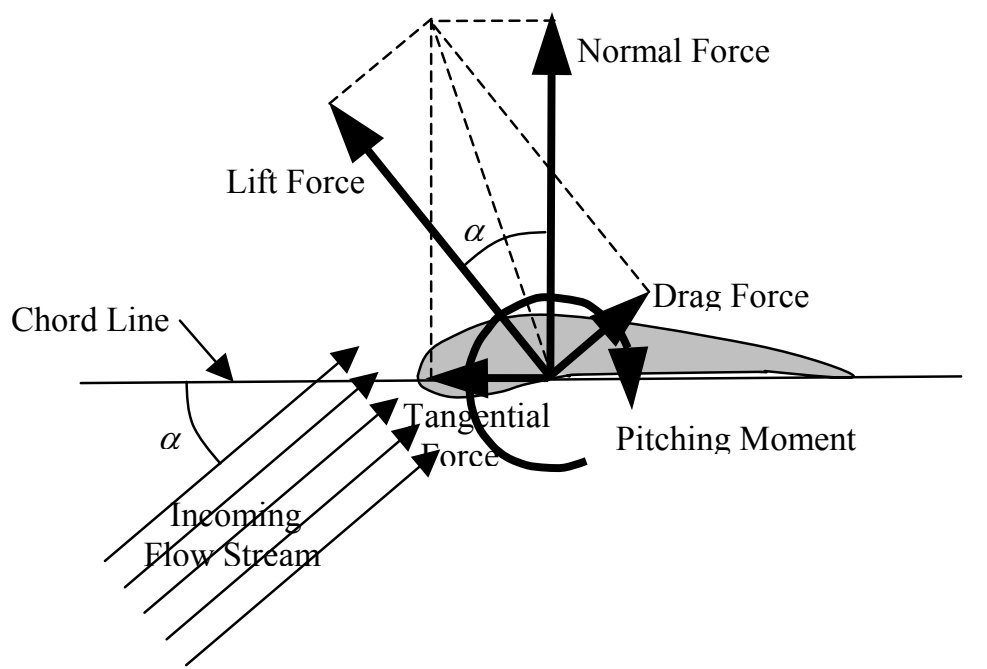

Figure 2.7: Resultant forces exerted on an airfoil

The relationships between the resultant forces are:

$$
\begin{gathered}
\text { Normal Force }=\text { Lift Force } \times \cos (\alpha)+\text { Drag Force } \times \sin (\alpha) \\
\text { and } \\
\text { Tangential Force }=\text { Lift Force } \times \sin (\alpha)-\operatorname{Drag} \text { Force } \times \cos (\alpha)
\end{gathered}
$$

or equivalently:

$$
\begin{gathered}
\text { Lift Force }=\text { Normal Force } \times \cos (\alpha)+\text { Tangential Force } \times \sin (\alpha) \\
\text { and } \\
\text { Drag Force }=\text { Normal Force } \times \sin (\alpha)-\text { Tangential Force } \times \cos (\alpha)
\end{gathered}
$$

The resultant forces are normally characterized by dimensionless coefficients. The lift and drag coefficients, $C_{L}$ and $C_{D}$ respectively, are defined as:

$$
C_{L}=\frac{\text { Lift Force / Unit Span }}{\frac{1}{2} \rho V_{r e l}^{2} c}
$$

and 


$$
C_{D}=\frac{\text { Drag Force } / \text { Unit Span }}{\frac{1}{2} \rho V_{\text {rel }}^{2} c}
$$

where $V_{r e l}$ is the velocity of the incoming flow stream relative to the airfoil and $c$ is the chord length, the distance between the leading edge and trailing edge measured along the chord line. In blade element theory, the lift and drag forces per unit span employed in these equations can be thought of as the differential lift and drag forces acting on the blade element in question divided by the differential length of the element, $d r$.

Similarly, the dimensionless normal and tangential force coefficients, $C_{N}$ and $C_{T}$ respectively, are defined as:

$$
\begin{gathered}
C_{N}=\frac{\text { Normal Force / Unit Span }}{\frac{1}{2} \rho V_{r e l}^{2} c} \\
\text { and } \\
C_{T}=\frac{\text { Tangential Force / Unit Span }}{\frac{1}{2} \rho V_{r e l}^{2} c}
\end{gathered}
$$

Finally, the dimensionless pitching moment coefficient, $C_{M}$, is defined as:

$$
C_{M}=\frac{\text { Pitching Moment } / \text { Unit Span }}{\frac{1}{2} \rho V_{r e l}^{2} c^{2}}
$$

In the denominator of each coefficient is the common product $1 / 2 \rho V_{\text {rel }}^{2}$, commonly referred to as the dynamic pressure, QNORM:

$$
Q N O R M=\frac{1}{2} \rho V_{r e l}^{2}
$$

These dimensionless coefficients quantitatively describe how flow past an airfoil of a given profile will exert forces on the airfoil. The dimensionless coefficients are heavily dependent on the airfoil profile and angle of attack and to a lesser, but generally substantial extent, on the size of the airfoil and the relative speed of the incoming flow. The dependence of the dimensionless coefficients to the size of airfoil and the relative speed are generally quantified using the nondimensional Reynolds number, $R e$, defined as the ratio of inertia forces to viscous forces: 


$$
\operatorname{Re}=\frac{\rho V_{r e l} c}{\mu}
$$

where $\mu$ is the viscosity of the fluid.

For any given airfoil, the dimensionless coefficient airfoil data are typically presented in a graphical format as plots of the coefficients versus angle of attack. A different set of curves is needed for each Reynolds number considered. An example set of dimensionless coefficient airfoil data is given in Tables 5.2-5.7 and Figs. 5.1 and 5.2 for the S809 airfoil used on the UAE wind turbine, which is analyzed later. These airfoil data are obtained by testing scaled-down airfoil sections in a wind tunnel where flow conditions can be carefully controlled and twodimensional flow can be closely approximated.

At low angles of attack, the dimensionless lift coefficient increases linearly with angle of attack and drag is reasonably small. Flow is attached to the airfoil throughout this regime. At an angle of attack of roughly $6^{\circ}$ or $7^{\circ}$, the flow on the upper surface of the airfoil begins to separate and a condition known as stall begins to develop. The dimensionless lift coefficient peaks and the dimensionless drag coefficient increases as stall increases. Dimensionless coefficient airfoil data are generally unavailable for angles of attack much beyond stall. This unavailability is unfortunate for a wind turbine analyst since wind turbine airfoils do operate in this regime, especially under heavy winds and yawed conditions. In the post-stall regime, the flow becomes decreasingly dependent on the airfoil profile and behaves similarly to high angle of attack flow around flat plates. Using this notion, models have been developed that are commonly used to extrapolate the available airfoil data across the entire $360^{\circ}$ range of potential angles of attack. The most popular Viterna-Corrigan post-stall model is developed in Viterna and Corrigan (1981) and summarized in Eggleston and Stoddard (1987) and Hansen and Laino (1998). A detailed description of the dimensionless pitching moment coefficient is beyond the scope of this work.

The differential rotor thrust, $d T$, and a differential rotor torque, $d Q$, acting on each blade element as described by blade element theory can be found by analyzing the geometry of Fig. 2.8. In this figure, the blade is specified as propagating to the left as a result of blade rotation. The pitching moment is absent because it contributes nothing to the rotor thrust and torque.

In this figure, $\theta_{P}$ is the blade collective pitch angle measured relative to the point of zero twist, $\theta_{T}$ is the local blade twist angle, and $\theta_{P T}$ is their sum. The local blade twist angle is a function of the annular radius and does not change with time (assuming the blade is structurally rigid and does not feather). The blade collective pitch angle can change with time to adjust for different operating conditions (for a pitch-regulated wind turbines only), but is constant across the entire length of the blade (the rigid blade is connected at the root by a pitching mechanism). The angle $\theta$ is the angle of the relative incoming flow stream with respect to the plane of rotation and is equal to the sum of $\theta_{P T}$ and $\alpha$. 


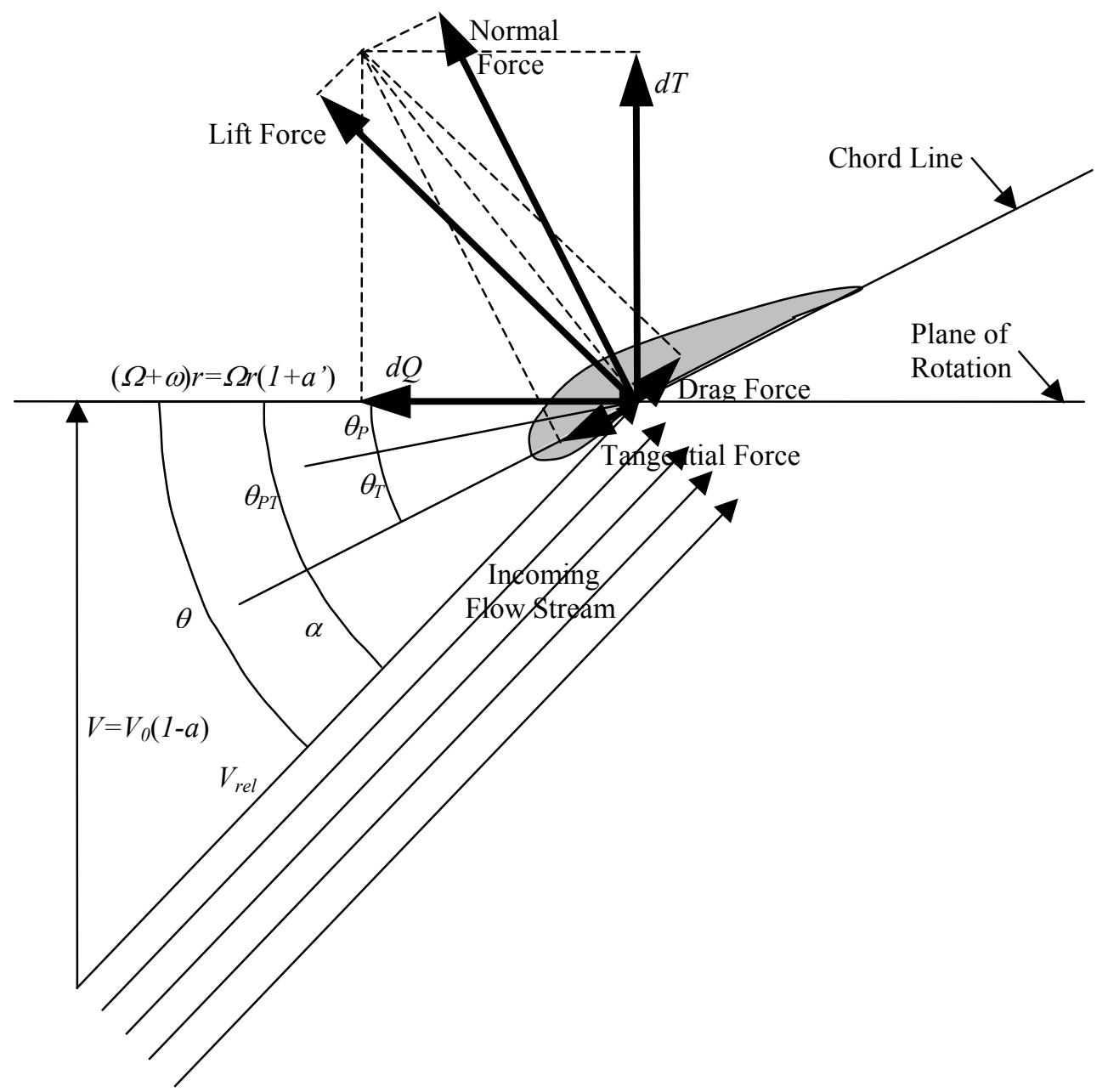

Figure 2.8: Blade element theory geometry

The velocity of the incoming flow stream relative to the blade element, $V_{\text {rel }}$, is the vector sum of the axial inflow velocity at the rotor plane, $V$, the inflow velocity caused by the rotation of the blade, $\Omega r$, and the inflow velocity caused by wake rotation at the rotor plane, $\omega r$ ( $r$ is the radius of the blade element whose cross section is shown in the figure). With the help of Eqs. (2.10) and (2.34), $V_{\text {rel }}$ is found to be:

$$
V_{r e l}=\sqrt{\left[V_{0}(1-a)\right]^{2}+\left[\Omega r\left(1+a^{\prime}\right)\right]^{2}}
$$

Also, using geometry, a relationship can be developed relating $a, a^{\prime}$, and $\theta$ :

$$
\tan (\theta)=\frac{V_{0}(1-a)}{\Omega r\left(1+a^{\prime}\right)}
$$


Similarly, a relationship can be developed relating $V_{\text {rel }}, a$, and $\theta$.

$$
V_{r e l}=\frac{V_{0}(1-a)}{\sin (\theta)}
$$

The relationships between the resultant forces are:

$$
\begin{gathered}
d T=\operatorname{Lift} \text { Force } \times \cos (\theta)+\text { Drag Force } \times \sin (\theta) \\
\text { and } \\
d Q=r[\text { Lift Force } \times \sin (\theta)-\text { Drag Force } \times \cos (\theta)]
\end{gathered}
$$

or equivalently:

$$
\begin{gathered}
d T=\operatorname{Normal~Force~} \times \cos \left(\theta_{P T}\right)-\text { Tangential Force } \times \sin \left(\theta_{P T}\right) \\
\text { and } \\
d Q=r\left[\text { Normal Force } \times \sin \left(\theta_{P T}\right)+\text { Tangential Force } \times \cos \left(\theta_{P T}\right)\right]
\end{gathered}
$$

To be precise, the lift, drag, normal, and tangential forces shown in Fig. 2.8 and used in the equations represent the differential components of the force imparted by the flow stream on the blade element whose cross section is shown. Also, all the differential forces shown in Fig. 2.8 and used in the equations represent the differential forces acting on a single blade. If the wind turbine rotor consists of $B$ identical blades, the differential rotor thrust, $d T$, and differential rotor torque, $d Q$, equal the following when substituting the dimensionless coefficients for the forces:

$$
\begin{gathered}
d T=B \frac{1}{2} \rho V_{r e l}^{2}\left[C_{L} \cos (\theta)+C_{D} \sin (\theta)\right] c d r \\
\text { and } \\
d Q=B \frac{1}{2} \rho V_{r e l}^{2}\left[C_{L} \sin (\theta)-C_{D} \cos (\theta)\right] c r d r
\end{gathered}
$$

or equivalently:

$$
d T=B \frac{1}{2} \rho V_{r e l}^{2}\left[C_{N} \cos \left(\theta_{P T}\right)-C_{T} \sin \left(\theta_{P T}\right)\right] c d r
$$

and 


$$
d Q=B \frac{1}{2} \rho V_{r e l}^{2}\left[C_{N} \sin \left(\theta_{P T}\right)+C_{T} \cos \left(\theta_{P T}\right)\right] c r d r
$$

$d T$ and $d Q$ now represent the total differential thrust and differential torque, respectively, acting on an annular ring of radius $r$ and thickness $d r$, just as they were represented in sections 2.2 and 2.3. Moreover, just as the induction factors and velocities are functions of the annular radius as in section 2.3, the dimensionless coefficients and the chord length used in the previous equations are all permitted to be functions of the annular radius.

\subsection{Blade Element Momentum Theory and Performance Prediction}

Combining the results of the previous analyses enables one to model the performance of a rotor whose airfoil properties, size, and twist distribution are known. This type of analysis is BEM theory, which is based on the fact that the differential rotor thrust, $d T$, and differential rotor torque, $d Q$, derived from momentum theory and blade element theory must be equivalent.

Before these equivalency relationships are applied, the momentum theory equations are customarily adjusted to account for the finite number of blades that are inherent in real wind turbine rotors (the number of blades, $B$, cannot be infinite). The most common adjustment is the straightforward tip loss (or correction) factor, $F$, developed by Prandtl as summarized by Wilson et al. (1976). Although originally developed to account for the finite number of blades, as the name suggests, the tip loss factor additionally accounts for the finiteness of the blades (the radius of the blades, $R$, cannot be infinite). Because a blade has a suction surface and a pressure surface, air tends to flow over the blade tip from the lower (pressure) surface to the upper (suction) surface, effectively reducing the resulting forces in the vicinity of the tip. The tip loss factor is defined as:

$$
F=\left(\frac{2}{\pi}\right) \cos ^{-1}\left[e^{-\frac{B(R-r)}{2 r \sin (\theta)}}\right]
$$

where the resulting inverse cosine term is assumed to be expressed in radians. $F$ is very near unity except when $B$ is small, $\theta$ is large, or $r$ approaches $R$, in which case $F$ tends toward zero.

Prandtl's tip loss factor is incorporated into the momentum theory equations [Eqs. (2.24) and (2.36)]:

$$
\begin{gathered}
d T=F \rho V_{0}^{2} 4 a(1-a) \pi r d r \\
\text { and } \\
d Q=F \rho V_{0} \Omega r^{2} 4 a^{\prime}(1-a) \pi r d r
\end{gathered}
$$

The tip loss factor does not affect the blade element theory equations. 
Although less common, a hub loss factor may also be incorporated into the momentum theory equations. Incorporating Prandtl's hub loss factor is similar to incorporating the tip loss factor, but is beyond the scope of this work. Eggleston and Stoddard (1987) describe this model.

Equating the differential rotor thrust from moment theory [Eq. (2.64)] and blade element theory [Eq. (2.59)] and incorporating the value of $V_{r e l}$ from Eq. (2.54) results in:

$$
\left(\frac{a}{1-a}\right)\left[\frac{\sin ^{2}(\theta)}{\cos (\theta)}\right]=\left(\frac{B c}{2 \pi r}\right)\left(\frac{C_{L}}{4 F}\right)\left[1+\frac{C_{D} \sin (\theta)}{C_{L} \cos (\theta)}\right]
$$

Equating the differential rotor torque from moment theory [Eq. (2.65)] and blade element theory [Eq. (2.60)] and incorporating the value of $V_{r e l}$ from Eq. (2.54) results in:

$$
\left(\frac{\Omega r}{V_{0}}\right)\left[\frac{\sin (\theta)}{\cos (\theta)}\right]\left(\frac{a^{\prime}}{1-a}\right)=\left(\frac{B c}{2 \pi r}\right)\left(\frac{C_{L}}{4 F}\right)\left[\frac{1}{\cos (\theta)}-\frac{C_{D}}{C_{L} \sin (\theta)}\right]
$$

The first term on the right-hand side of each of these equations is known as the local solidity, $\sigma$.

$$
\sigma^{\prime}=\frac{B c}{2 \pi r}
$$

This variable is an expanded definition of the more commonly used rotor solidity, defined as the dimensionless number corresponding to the ratio of the total blade area to the total area swept by the rotor (rotor plane area):

$$
\sigma=\frac{\overline{B c}}{\pi R}
$$

Eq. (2.69) assumes that the average chord length of each blade is $\bar{c}$. This assumption is not employed in Eq. (2.68).

Eliminating $a$ ' from Eq. (2.67) using Eq. (2.53), then eliminating $a$ from Eq. (2.66) and the revised Eq. (2.67) results in:

$$
C_{L}=\left(\frac{4 F}{\sigma^{\prime}}\right) \sin (\theta)\left\{\frac{\cos (\theta)-\lambda_{r} \sin (\theta)}{\sin (\theta)+\lambda_{r} \cos (\theta)+\frac{C_{D}}{C_{L}}\left[\lambda_{r} \sin (\theta)-\cos (\theta)\right]}\right\}
$$

The local solidity $\left(\sigma^{\prime}\right)$ from Eq. (2.68) and the local speed ratio $\left(\lambda_{r}\right)$ from Eq. (2.38) have been employed when developing Eq. (2.70). 
Accepted practice is to set the dimensionless drag coefficient, $C_{D}$, equal to zero when analyzing the performance of a given wind turbine. For wind turbines that consist of aerodynamically effective blades operating in a condition where drag is small, this simplification introduces negligible errors. With $C_{D}$ equal to zero, Eqs. (2.66), (2.67), and (2.70) simplify to:

$$
\begin{gathered}
\left(\frac{a}{1-a}\right)\left[\frac{\sin ^{2}(\theta)}{\cos (\theta)}\right]=\frac{\sigma^{\prime} C_{L}}{4 F} \\
\lambda^{\prime}\left[\frac{\sin (\theta)}{\cos (\theta)}\right]\left(\frac{a^{\prime}}{1-a}\right)=\left(\frac{\sigma^{\prime} C_{L}}{4 F}\right)\left[\frac{1}{\cos (\theta)}\right] \\
\text { and } \\
C_{L}=\left(\frac{4 F}{\sigma^{\prime}}\right) \sin (\theta)\left[\frac{\cos (\theta)-\lambda_{r} \sin (\theta)}{\sin (\theta)+\lambda_{r} \cos (\theta)}\right]
\end{gathered}
$$

Note that $\sigma^{\prime}$ and $\lambda_{r}$ have again been employed when developing Eqs. (2.71) and (2.72).

Equation (2.73) explicitly expresses the lift coefficient $\left(C_{L}\right)$ as a function of the angle of attack $(\alpha)$ at a specific annular radius $(r)$ for a wind turbine operating at a constant wind speed $\left(V_{0}\right)$ whose number of blades $(B)$, blade radius $(R)$, pitch $\left(\theta_{P}\right)$, twist distribution $\left(\theta_{T}\right)$, chord distribution $(c)$, and rotational speed $(\Omega)$ are fixed. Equation (2.73) is BEM theory's prediction of the feasible $C_{L}$ versus $\alpha$ operating points for a fixed wind turbine operating at a static operating condition.

Equation (2.73) is not a function of the airfoil profile of the blades, which would be manifested in the dimensionless coefficient airfoil data $\left(C_{L}\right.$ and $C_{D}$ or equivalently $C_{N}$ and $\left.C_{T}\right)$. To see this, the reader should recall that $\alpha$ is incorporated into $\theta$ and $F ; r$ is incorporated into $F, \sigma$, and $\lambda_{r}$; $V_{0}$ is incorporated into $\lambda_{r} ; B$ is incorporated into $F$ and $\sigma^{\prime} ; R$ is incorporated into $F ; \theta_{P}$ and $\theta_{T}$ are incorporated into $\theta$ and $F$; $c$ is incorporated into $\sigma^{\prime} ; \Omega$ is incorporated into $\lambda_{r}$; and that no other variables are inherent in Eq. (2.73). Thus, the curve presented by Eq. (2.73) is valid for any wind turbine operating at a condition where drag is small and the parameters are fixed.

For a wind turbine whose blade airfoil profiles are fixed along with the various parameters specified previously, BEM theory predicts that the actual aerodynamic operating condition (lift coefficient and angle of attack pairs) at each blade element will be the point where the BEM $C_{L}$ versus $\alpha$ curve [Eq. (2.73)] intersects the $C_{L}$ versus $\alpha$ curve characterizing the aerodynamic properties of the given airfoil. This notion is a manifestation of assumption (2) in section 2.4. The intersection point can be found either graphically (easiest for hand-computations) or numerically (iteratively), which is easiest for computer-based solutions. In practice, the iterative method is usually employed with Eqs. (2.71), (2.72), and (2.53) instead of Eq. (2.73). An example of the graphical solution is presented in Chapter 6. The graphical solution enhances the visibility of the weaknesses in the model. 
Once the lift coefficient and angle of attack are found for each blade element, the total aerodynamic forces exerted on the blades by the flow stream can be found by simple integration (or by a simple summation approximating the integral for the case when only a finite number of blade elements are used). This enables one to determine the overall power output of the wind turbine as well as the aerodynamic loads experienced by its various members. A derivation of the overall rotor power coefficient is presented next.

The power extracted from the wind by the rotor, $P$, employed in the definition of the nondimensional power coefficient, $C_{P}$, of Eq. (2.14), may be found:

$$
P=\int \Omega d Q
$$

Exactly as stated in the paragraph following Eq. (2.26), the product of the differential rotor torque, $d Q$, and the angular speed of the rotor, $\Omega$, in Eq. (2.74) represents the differential power extracted by the turbine, $d P$.

Using the differential rotor torque from blade element theory [Eq. (2.60)] and incorporating the value of $V_{\text {rel }}$ from Eq. (2.54), Eq. (2.74) can be rewritten:

$$
P=\int_{H}^{R} \Omega B \frac{1}{2} \rho\left[\frac{V_{0}(1-a)}{\sin (\theta)}\right]^{2}\left[C_{L} \sin (\theta)-C_{D} \cos (\theta)\right] c r d r
$$

where $H$ is the radius of the rotor hub. Although Eq. (2.73), describing BEM theory's prediction of the feasible $C_{L}$ versus $\alpha$ operating points for a fixed wind turbine operating at a static operating condition, is found assuming drag is small and thus negligible. This expression for the total power extracted by the rotor includes drag. This too is common practice.

Substituting this expression for the total power extracted by the rotor into the equation for dimensionless power coefficient [Eq. (2.14)] and simplifying with help from Eq. (2.68) for the local solidity, Eq. (2.38) for the local speed ratio, and Eq. (2.39) for the tip speed ratio results in:

$$
C_{P}=\frac{2}{\lambda R} \int_{H}^{R} \frac{\sigma^{\prime} \lambda_{r}^{2}(1-a)^{2} C_{L}}{\sin ^{2}(\theta)}\left[\sin (\theta)-\frac{C_{D} \cos (\theta)}{C_{L}}\right] d r
$$

Eliminating $a$ ' from Eq. (2.67) using Eq. (2.53), then eliminating $a$ from Eq. (2.76) and the revised Eq. (2.67) results in:

$$
C_{P}=\frac{2}{\lambda R} \int_{H}^{R} \frac{\sigma^{\prime} \lambda_{r}^{2} C_{L}}{\sin ^{2}(\theta)}\left[\frac{\lambda_{r}^{2} \sin ^{2}(\theta)}{\cos ^{2}(\theta)-\frac{\sigma^{\prime} C_{L} \cos (\theta)}{2 F}+\frac{\left(\sigma^{\prime}\right)^{2} C_{L}^{2}}{16 F^{2}}}\right]\left[\sin (\theta)-\frac{C_{D} \cos (\theta)}{C_{L}}\right] d r(2.77)
$$


Equation (2.77) can be simplified by substituting the value of $C_{L}$ from Eq. (2.73) into all but the rightmost $C_{L}$ in Eq. (2.77). This action results in:

$$
C_{P}=\frac{2}{\lambda R} \int_{H}^{R} \lambda_{r}^{2} \sin ^{2}(\theta)\left[\cos (\theta)-\lambda_{r} \sin (\theta)\right]\left[\sin (\theta)+\lambda_{r} \cos (\theta)\right]\left[1-\frac{C_{D} \cos (\theta)}{C_{L} \sin (\theta)}\right] d r
$$

\subsection{Advanced Aerodynamics Topics}

BEM theory assumes that the flow field around the wind turbine rotor is in equilibrium at every time step considered (quasi-equilibrium). During the operation of a real wind turbine, however, the flow field is always changing, even during normal operation. Consequently, BEM theory computations may not reflect reality when examining unsteady events. The AeroDyn aerodynamics subroutines employed in the FAST_AD design code use the BEM theory with modifications to improve the results for unsteady events. This section briefly highlights advanced aerodynamics topics that address these conditions.

Simple uniform wind shear is modeled using a power-law profile, in which the incoming wind speed, $V_{0}$, becomes dependent on the elevation above the ground. Horizontal variation in incoming wind speed is usually modeled with a linear velocity profile. Wind-inflow data are usually stored in files that permit modelers to specify wind parameters that change with time (time-series inputs). Alternatively, several models have been developed to simulate full field turbulent flow. In these models, inflow data can have velocity components in three dimensions that vary across the entire rotor plane. These velocity components have an effect on the local inflow angles.

For downwind rotor configurations, aerodynamic loads on the blades are affected by the tower wake, commonly called the tower shadow. The tower shadow or wake is modeled as a reduction in wind velocity according to a cosine law characterized by the width of the flow stream at the rotor plane exhibiting a velocity deficit and the velocity deficit at the center of the wake.

When wind directions change, or when the rotor exhibits a yaw error, the axial induction is affected by both a cyclic variation in axial flow velocities relative to the blades and a deflected rotor wake. The cyclic variation in axial flow is accounted for by including the axial component of the blade velocity in the equations for the relative velocity of the incoming flow stream. The deflected wake is accounted for by multiplying the axial induction factor, $a$, by a so-called skewed-wake correction factor as described by Wilson et al. (1999).

Pressure gradients develop across the blade span in the three-dimensional flow environment of rotary wing aerodynamics. Ensuing spanwise flow velocity helps maintain attached flow on the inboard parts of the blades, delaying stall and increasing lift. These so-called three-dimensional stall delay effects are accounted for by modifying the aerodynamic force coefficient-angle of attack relationships in the stall region for the inboard blade elements. Du and Selig (1998) outline the most popular stall delay model.

For blades that are downwind of the tower, turbines that are operating in yawed conditions, or even turbines operating in a turbulent flow, the aerodynamic environment is rapidly changing. During such dynamic conditions, stall may be delayed or may take place earlier than expected 
(known as dynamic stall). The most common dynamic stall model is the Beddoes-Leishman model, summarized by Leishman and Beddoes (1989).

In turbulent conditions, the extended flow field around the rotor cannot react promptly enough to establish steady-state conditions instantaneously. Thus, the aerodynamic conditions at the rotor are not necessarily the conditions seen just ahead of the rotor. The response of the extended flow field to turbulent events is referred to as dynamic inflow. An overview of the popular Pitt and Peters dynamic inflow model is given in Suzuki and Hansen (1998). 


\section{Structural Modeling Theory}

Once wind-inflow conditions are correlated to applied loads on the wind turbine, structural models are needed to accurately predict and understand the complex interactions between their dynamically active members. Accurate structural models are thus essential to successfully design and analyze wind energy systems.

The subsequent analysis develops the fundamental structural models employed in the FAST_AD design code for two-bladed HAWTs. The modifications needed to extend the model to threebladed HAWTS are beyond the scope of this work. Wind turbine geometry, coordinate systems, and degrees of freedom (DOFs) are first discussed in section 3.1. Since FAST_AD models the blades and tower as flexible bodies, their deflections are presented next in section 3.2.

Expressions relating to the kinematics and kinetics of wind turbine motion are developed in sections 3.3 and 3.4, respectively. Finally, Kane's equations of motion, which describe the force-acceleration relationships of the entire wind turbine system, are presented in section 3.5. Discrepancies between the models developed herein and those given by Wilson et al. (1999) and implemented in the FAST_AD design code and the Modes preprocessor code are noted using footnotes where appropriate. This chapter is devoted entirely to the structural models employed in the FAST_AD design code. Structural models, such as the commonly used equivalent-springhinge models of flexible blades, though simpler, are beyond the scope of this work.

\subsection{Geometry, Coordinate Systems, and Degrees of Freedom}

The FAST_AD design code models a wind turbine structurally as a combination of six rigid and four flexible members. The six rigid bodies are the Earth, nacelle, tower-top base plate, armature, hub, and gears. The four flexible bodies are the two blades, tower, and drive shaft. The model connects these bodies through 15 DOFs. Blade deflections account for six DOFs: two arise from the first flapwise, two from the second flapwise, and two from the first edgewise natural vibration mode of each blade. Tower deflections account for four DOFs: the first two natural vibration modes in each longitudinal and lateral direction. Rotor teeter, rotor speed variation, drive train flexibility, and nacelle yaw and tilt account for the remaining five DOFs. Each blade can be regarded as having a structural pretwist, but no torsional freedom is modeled.

FAST_AD employs several reference frames for ease in conceptualizing geometry and developing kinematics and kinetics expressions. Most reference frames ${ }^{6}$, corresponding to a coordinate system formed by a dextral set of orthogonal unit vectors (denoted by a bold lowercase script letter) are fixed in one of the rigid bodies (denoted by an uppercase character).

\footnotetext{
${ }^{6}$ Wilson et al. (1999) claim that every reference frame is fixed in one of these six rigid bodies. In particular, reference frame $\boldsymbol{d}$ is claimed to be fixed in the rigid nacelle $(\mathrm{N})$. However, this claim can only be true if the nacelle tilt, represented by DOF variable $q_{5}$ is held constant. FAST_AD code developers should consider revising this statement.
} 
These coordinate systems are listed in Table $3.1^{7}$. A number of points on the wind turbine are also labeled for convenience. These are listed in Table 3.2.

\section{Table 3.1: Coordinate System Descriptions ${ }^{7}$}

\begin{tabular}{|c|l|l|}
\hline $\begin{array}{c}\text { Unit Vector } \\
\text { Triad }\end{array}$ & Body Fixed In & Description \\
\hline $\boldsymbol{a}$ & E (Earth) & Inertial coordinates \\
\hline $\boldsymbol{b}$ & B (Base plate) & Tower-top coordinates \\
\hline $\boldsymbol{d}$ & N/A & Yaw coordinates \\
\hline $\boldsymbol{c}$ & N (Nacelle) & Tilt coordinates \\
\hline $\boldsymbol{e}$ & L (Low speed shaft) & Azimuth coordinates \\
\hline $\boldsymbol{f}$ & H (Hub / rotor) & Teeter coordinates \\
\hline $\boldsymbol{g}$ & H (Hub / rotor) & Delta-3 coordinates \\
\hline $\boldsymbol{i}$ & H (Hub / rotor) & Coning coordinates \\
\hline
\end{tabular}

Table 3.2: System Points

\begin{tabular}{|c|c|l|}
\hline Point & Body Fixed In & Description \\
\hline O & B (Base plate) & Tower-top \\
\hline D & N (Nacelle) & Nacelle center of mass \\
\hline P & L (Low speed shaft) & Teeter pin \\
\hline Q & H (Hub / rotor) & Intersection of blade inertia axes \\
\hline C & H (Hub / rotor) & Hub center of mass \\
\hline S & Blade element & Center of blade element in deflected blade \\
\hline S' & H (Hub / rotor) & Center of blade element in undeflected blade \\
\hline T & Tower element & Center of tower element in deflected tower \\
\hline
\end{tabular}

The variable names of each DOF used by FAST_AD are listed in Table $3.3^{8}$. Other commonly used coordinates are listed in Table 3.4 (for angles ${ }^{9}$ ) and Table 3.5 (for distances).

${ }^{7}$ This table differs from the corresponding table given in Wilson et al. (1999), not just from the discrepancy of reference frame $\boldsymbol{d}$ indicated previously. The bodies containing the reference frames $\boldsymbol{e}, \boldsymbol{f}, \boldsymbol{g}$, and $\boldsymbol{i}$ are also different. The corresponding table given in Wilson et al. (1999) is in error since reference frame $\boldsymbol{e}$ cannot be fixed in the hub as listed if any teeter motion is taking place. Teetered rotors consist of blades rigidly attached to a hub that teeters about a pin located on the low speed shaft. If teeter motion is taking place, reference frame $\boldsymbol{e}$ can be considered fixed on the rotor side of the low speed shaft and reference frames $\boldsymbol{f}, \boldsymbol{g}$, and $\boldsymbol{i}$ can be considered fixed in the teetering hub (rotor). FAST_AD code developers should consider revising this table.

${ }^{8}$ This table differs from the corresponding table given in Wilson et al. (1999) by the description of the DOF variable $q_{15}$ (azimuth angle, generator side). Wilson et al. (1999) ) claim that $q_{15}$ represents the angle of the high-speed shaft (i.e., the generator side of the gearbox) when in fact FAST_AD models $q_{15}$ as the angle of the low-speed shaft near the entrance to the gearbox. That is, the only difference between the parameter $q_{15}$ and $q_{4}$ in FAST_AD is the twist of the low-speed shaft because of its torsional flexibility. The angle of the high-speed shaft should be roughly $n$ times the angle of the low-speed shaft, where $n$ is the gearbox ratio. FAST_AD code developers should consider revising this table.

${ }^{9}$ This table differs from the corresponding table given in Wilson et al. (1999) by the definition of the distributed structural pretwist angle, $\theta_{S}(r)$. The corresponding table given in Wilson et al. (1999) is in error since the distributed 
Table 3.3: Degree of Freedom Variables ${ }^{8}$

\begin{tabular}{|c|c|c|}
\hline Variable & $\begin{array}{c}\text { Direction / } \\
\text { Rotation Axis }\end{array}$ & Description \\
\hline$q_{1}$ & \multirow{2}{*}{$\begin{array}{l}\text { localized blade } \\
\text { coordinates }\end{array}$} & Blade 1 flapwise tip displacement for natural mode 1 \\
\hline$q_{2}$ & & Blade 2 flapwise tip displacement for natural mode 1 \\
\hline$q_{3}$ & $e_{2}=f_{2}$ & Teeter angle \\
\hline$q_{4}$ & $c_{1}=e_{1}$ & Azimuth angle, rotor side of drive train \\
\hline$q_{5}$ & $d_{3}=c_{3}$ & Nacelle tilt angle \\
\hline$q_{6}$ & $b_{2}=d_{2}$ & Nacelle yaw angle \\
\hline$q_{7}$ & $a_{1}$ & Longitudinal tower top displacement for natural mode 1 \\
\hline$q_{8}$ & $a_{3}$ & Latitudinal tower top displacement for natural mode 1 \\
\hline$q_{9}$ & $\overline{a_{1}}$ & Longitudinal tower top displacement for natural mode 2 \\
\hline$q_{10}$ & $a_{3}$ & Latitudinal tower top displacement for natural mode 2 \\
\hline$q_{11}$ & \multirow{2}{*}{$\begin{array}{l}\text { localized blade } \\
\text { coordinates }\end{array}$} & Blade 1 flapwise tip displacement for natural mode 2 \\
\hline$q_{12}$ & & Blade 2 flapwise tip displacement for natural mode 2 \\
\hline$q_{13}$ & \multirow{2}{*}{$\begin{array}{c}\text { localized blade } \\
\text { coordinates }\end{array}$} & Blade 1 edgewise tip displacement for natural mode 1 \\
\hline$q_{14}$ & & Blade 2 edgewise tip displacement for natural mode 1 \\
\hline$q_{15}$ & $c_{1}=e_{1}$ & $\begin{array}{l}\text { Azimuth angle, generator side of drive train, low speed shaft } \\
\text { side of gearbox }\end{array}$ \\
\hline
\end{tabular}

Table 3.4: Other Angle Variables ${ }^{9}$

\begin{tabular}{|c|c|l|}
\hline Angle & $\begin{array}{c}\text { Rotation } \\
\text { Axis }\end{array}$ & Description \\
\hline$\theta_{7}$ & $\boldsymbol{a}_{\mathbf{3}}$ & Longitudinal tower-top rotation \\
\hline$\theta_{8}$ & $\boldsymbol{a}_{\boldsymbol{1}}$ & Latitudinal tower-top rotation \\
\hline$z(4)$ & $\boldsymbol{c}_{\boldsymbol{1}}=\boldsymbol{e}_{\boldsymbol{1}}$ & Blade 1 zero azimuth offset \\
\hline$\delta_{3}$ & $\boldsymbol{f}_{\boldsymbol{1}}=\boldsymbol{g}_{\boldsymbol{1}}$ & Delta-3 angle (teeter hinge orientation) \\
\hline$\beta_{1}$ & $\boldsymbol{g}_{2}=\boldsymbol{i}_{2}$ & Blade 1 coning angle \\
\hline$\beta_{2}$ & $\boldsymbol{g}_{2}=\boldsymbol{i}_{2}$ & Blade 2 coning angle \\
\hline$\theta_{S}(r)$ & $-\boldsymbol{i}_{3}$ & Distibuted structural pretwist of each blade \\
\hline$\theta_{P}$ & $-\boldsymbol{i}_{3}$ & Blade collective pitch \\
\hline
\end{tabular}

structural pretwist angle cannot be a positive rotation about the $\boldsymbol{i}_{3}$-axis if it is to rotate the local principal axes to their proper orientation. A positive rotation about the $\boldsymbol{i}_{3}$-axis would twist the blade element toward stall, not feather. In actuality, the inboard blade elements should be twisted toward feather for maximum aerodynamic efficiency. In essence, the sign convention FAST_AD uses to define the blade structural pretwist angle causes FAST_AD to model the blades with a mirror image of the stiffness distribution physically relevant to the wind turbine being modeled. When running FAST_AD, a simple fix would be to reverse the sign of all of the distributed structural pretwist angles in the input file (i.e., if the structural twist of an inboard element should be $15^{\circ}$, then the input should be written as $-15^{\circ}$ in the input file). Unfortunately, that is not how Wilson et al. (1999) explain it to develop the input file. Consequently, users of FAST_AD will undoubtedly enter in the structural pretwist parameters incorrectly. FAST_AD code developers should consider revising the code to correct for this significant error! 
Table 3.5: Other Distance Variables

\begin{tabular}{|c|c|c|}
\hline Variable & Direction & Description \\
\hline$\overline{H_{S}}$ & $a_{2}$ & Height of the rigid base of the tower \\
\hline$H$ & $\overline{a_{2}}$ & Length of the flexible portion of the tower \\
\hline$H_{H}$ & $a_{2}$ & $\begin{array}{l}\text { Elevation of the hub (hub height) relative to the Earth's surface assuming } \\
\text { negligible nacelle tilt and tower deflection }\end{array}$ \\
\hline TWRHTOFFSET & $c_{2}$ & Distance between the hub and the tower-top base plate \\
\hline$u_{7}$ & $a_{1}$ & Total tower-top displacement in the longitudinal direction \\
\hline$u_{8}$ & $a_{3}$ & Total tower-top displacement in the lateral direction \\
\hline$D_{N}$ & $c_{1}=e_{1}$ & Distance between the tower-top base plate and the teeter pin \\
\hline$D_{N M 1}$ & $c_{1}=e_{1}$ & Distance between the tower-top base plate and the nacelle center of mass \\
\hline$D_{N M 2}$ & $c_{2}$ & Distance between the origin of $c$ and the nacelle center of mass \\
\hline$R_{U}$ & $-f_{1}=-g_{1}$ & Distance from the teeter pin to the blade axes intersection (undersling) \\
\hline$R_{U M}$ & $-f_{1}=-g_{1}$ & Distance from the teeter pin to the hub center of mass \\
\hline$R_{H}$ & $i_{3}$ & Hub radius; radius of the rigid portion of the rotor \\
\hline$R$ & $i_{3}$ & Total radius of the rotor \\
\hline
\end{tabular}

Relationships between the various coordinate systems, points, DOFs, and other angles and distances are illustrated graphically in Fig. 3.1. FAST_AD employs the convention that downwind displacements are positive displacements.

In FAST_AD, the bottom part of the tower can be modeled as rigid to a height $H_{S}$; thus, the length of the flexible part of the tower, $H$, is defined as:

$$
H=H_{H}-\text { TWRHTOFFSET }-H_{S}
$$

where $H_{H}$ is the elevation of the hub (hub height) relative to the Earth's surface and TWRHTOFFSET is the vertical distance between the hub and the tower-top base plate, both specified while assuming that the tower deflection and nacelle tilt are negligible.

The sums of the tip deflections for both natural modes in the longitudinal and lateral deflections form the total longitudinal and lateral displacements of the tower-top base plate, $u_{7}$ and $u_{8}$, respectively:

$$
\begin{array}{r}
u_{7}=q_{7}+q_{9} \\
\text { and } \\
u_{8}=q_{8}+q_{10}
\end{array}
$$




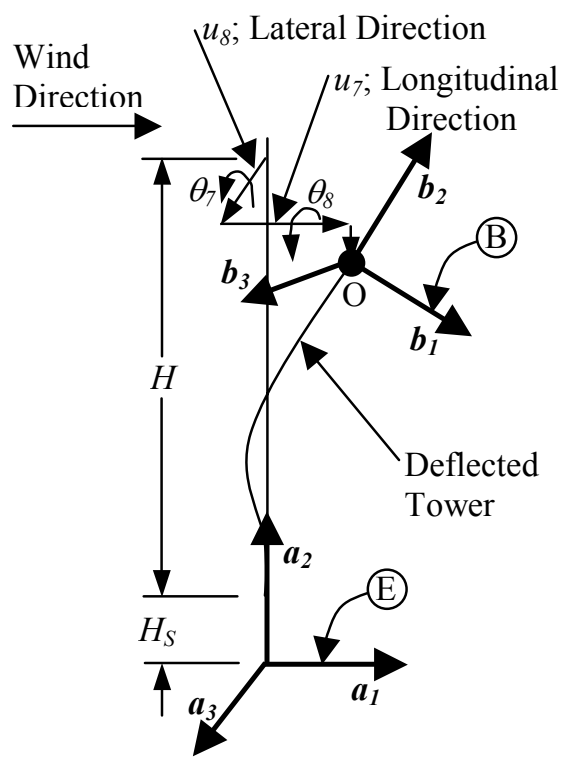

Tower Coordinates

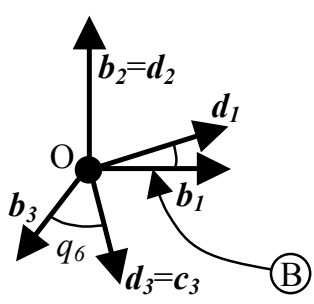

Base Plate Coordinates

Wind

Direction

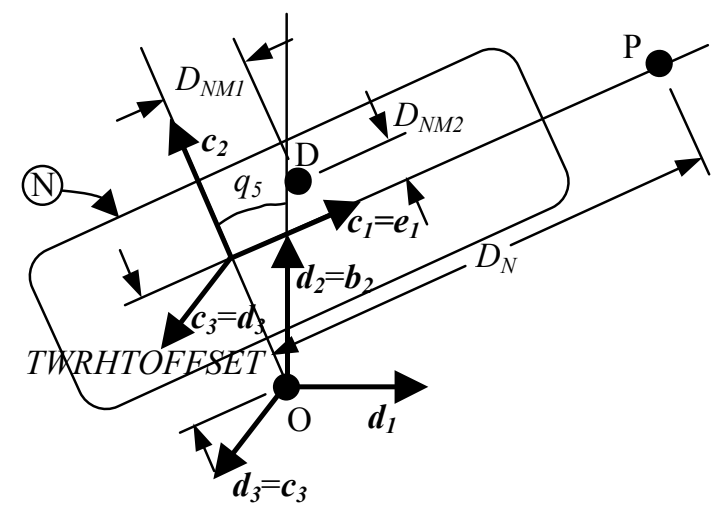

Nacelle Coordinates

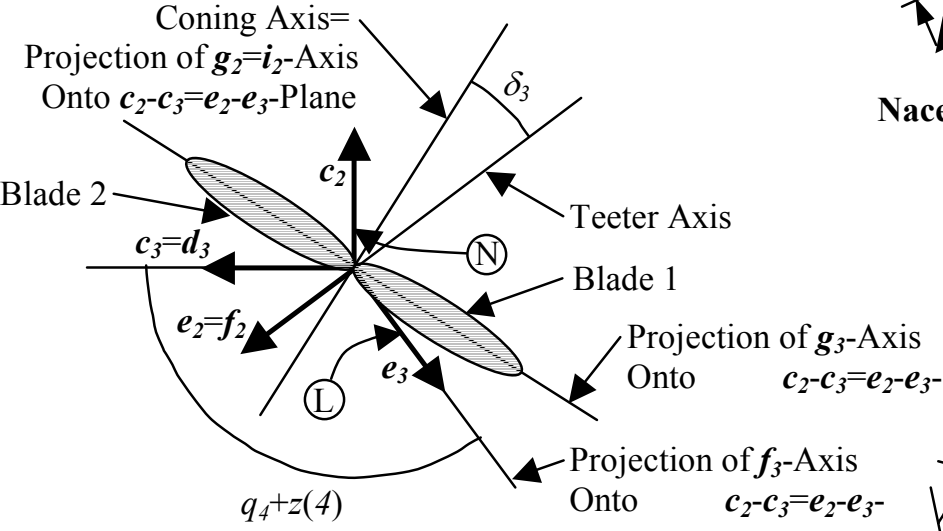

Azimuth Coordinates:

Looking Upwind From Downwind
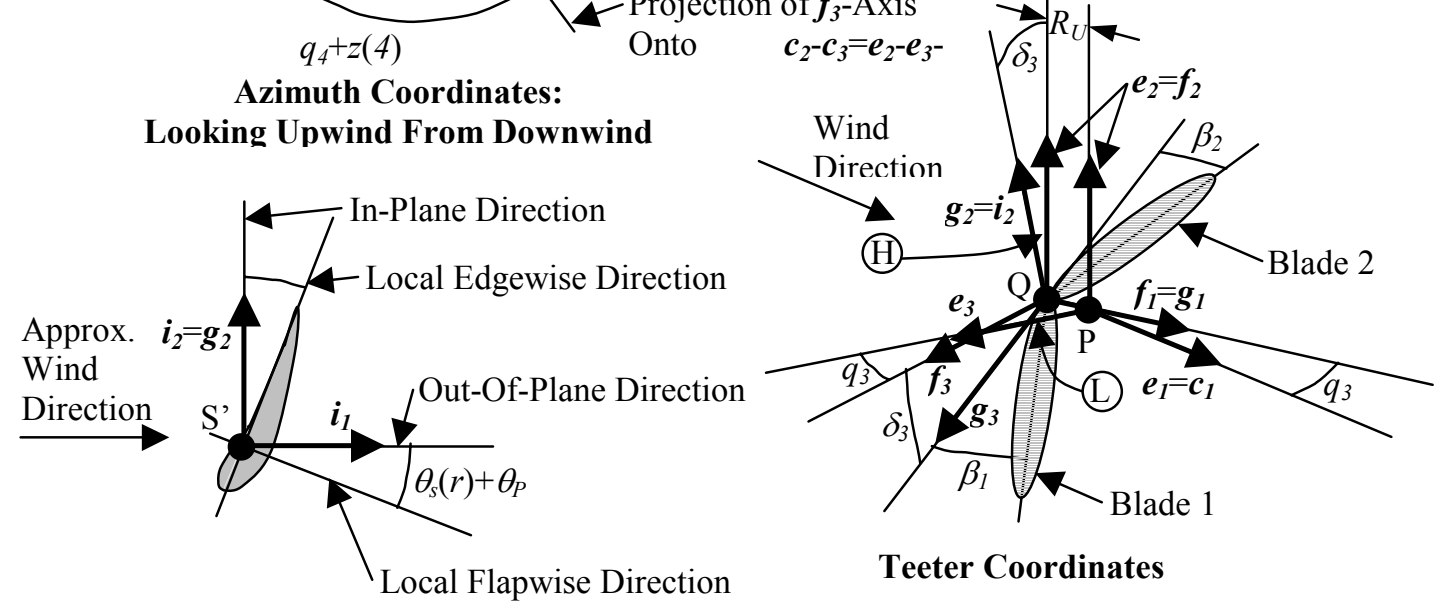

Teeter Coordinates

\section{Local Blade Element Coordinates}

Figure 3.1: FAST_AD coordinate system illustrations 
Since the generalized coordinates associated with the tip deflections are functions of time, so too are the total longitudinal and lateral displacements of the tower-top base plate.

In section 3.2, dealing with deflections of the tower and blades, the assumption is employed that the deflections are small. With this assumption, the tower-top rotations in both the longitudinal and lateral directions, $\theta_{7}$ and $\theta_{8}$ respectively, can be approximated:

$$
\begin{gathered}
\theta_{7}=-\left(\left.\frac{d \phi_{1 T}(h)}{d h}\right|_{h=H} q_{7}+\left.\frac{d \phi_{2 T}(h)}{d h}\right|_{h=H} q_{9}\right) \\
\text { and } \\
\theta_{8}=\left.\frac{d \phi_{1 T}(h)}{d h}\right|_{h=H} q_{8}+\left.\frac{d \phi_{2 T}(h)}{d h}\right|_{h=H} q_{10}
\end{gathered}
$$

where $\phi_{1 T}(h)$ and $\phi_{2 T}(h)$ are the first and second natural mode shapes of the tower, respectively. In these expressions, the elevation along the flexible part of the tower, $h$, ranges from zero to $H$. Note that $h$ equals zero at an elevation of $H_{S}$ relative to the Earth's surface. Also, the derivatives of the mode shapes are evaluated at an elevation of $h=H$ as indicated. The derivation of these natural mode shapes of the tower is presented in section 3.2 where the tower is assumed to deflect in the longitudinal and lateral directions independently; yet, the natural mode shapes in each direction are assumed to be identical in each direction. The negative sign is present in Eq. (3.4) since positive longitudinal displacements of the tower-top base plate tend to rotate the base plate about the negative $\boldsymbol{a}_{3}$-axis. The generalized coordinates associated with the tip deflections are functions of time; so are the longitudinal and lateral tower-top rotations of the plate.

Attached to the tower-top base plate is a yaw bearing $(\mathrm{O})$. The yaw bearing allows everything atop the tower to rotate $\left(q_{6}\right)$ as winds change direction. The yaw bearing also has the flexibility to allow everything atop the tower to tilt $\left(q_{5}\right)$ when responding to wind loads. The nacelle houses the generator and gearbox and supports the rotor. The center of mass of the nacelle (D) is related to the tower-top base plate by the position vector $\boldsymbol{r}^{O D 10}$ :

\footnotetext{
${ }^{10}$ This is in contrast to the position vector connecting the nacelle mass center to the tower-top base plate and the position vector connecting the teeter pin to the tower-top base plate employed by the FAST_AD design code:

$$
\begin{gathered}
\boldsymbol{r}^{O D}=D_{N M 1} \boldsymbol{c}_{\boldsymbol{1}}+D_{N M 2} \boldsymbol{c}_{\boldsymbol{2}} \\
\boldsymbol{r}^{O P}=D_{N} \boldsymbol{c}_{\boldsymbol{1}}
\end{gathered}
$$

as indicated by Wilson et al. (1999). These equations and the equations presented in the main body of the text are clearly in disagreement by the absence of the TWRHTOFFSET parameter in the former. The TWRHTOFFSET parameter is a recent addition to FAST_AD (version 3.00) and was added to improve the calculations of the resultant forces and moments about the tower-top base plate. Before the addition of this parameter, the tower-top base plate was assumed to be at the same elevation as the hub, which physically is not the case. Unfortunately, the TWRHTOFFSET parameter was only incorporated into the subroutines associated with the calculations of the output forces and moments about the tower-top base plate. The TWRHTOFFSET parameter is not used in any of the
} 


$$
\boldsymbol{r}^{O D}=D_{N M 1} \boldsymbol{c}_{1}+\left(D_{N M 2}+\text { TWRHTOFFSET }\right) \boldsymbol{c}_{2}
$$

Blade 1 is at an azimuth angle of $q_{4}$. The zero-azimuth reference position can be located by the azimuth offset parameter $z(4)$. Blade 2 is naturally $180^{\circ}$ out of phase with blade 1 . Drive train flexibility allows the induction generator to see an angular velocity that is different than $n$ times the angular velocity of the rotor, where $n$ is the gearbox ratio. The twist of the low-speed shaft is, because of its torsional flexibility, modeled with the $q_{15}$ parameter. The azimuth angle $q_{15}$, is essentially the sum of the azimuth angle, $q_{4}$, and the twist of the low-speed shaft.

If applicable to the wind turbine under consideration, teeter motion of the rotor is about a pin (P) fixed on the low-speed shaft. The position vector connecting the teeter pin to the tower-top base plate, $r^{O P}$, is ${ }^{10}$ :

$$
\boldsymbol{r}^{O P}=D_{N} \boldsymbol{c}_{1}+\text { TWRHTOFFSET } \boldsymbol{c}_{2}
$$

For an upwind turbine configuration, the distance between the tower-top base plate and the teeter pin in the $\boldsymbol{c}_{\boldsymbol{l}}=\boldsymbol{e}_{\boldsymbol{I}}$ direction, $D_{N}$, must be less than zero.

The delta- 3 angle, $\delta_{3}$, orients the axis of the unconed rotor blades so it is no longer perpendicular to the axis of the teeter pin. In this case, the teeter motion has both a flapping and a pitching component. If the delta-3 angle is zero, teeter motion is purely flapping motion.

Each blade can be coned a different amount ( $\beta_{1}$ for blade 1 and $\beta_{2}$ for blade 2 ), though the coning angles are constant, not changing with time. Coning begins in the very center of the hub at point $\mathrm{Q}$, which is offset of the teeter pin (P) by a distance $R_{U}$ along the central axis of the hub (undersling length). The position vector connecting the blade axes intersection point and the teeter pin, $\boldsymbol{r}^{P Q}$, is:

$$
\boldsymbol{r}^{P Q}=-R_{U} \boldsymbol{g}_{1}
$$

Located between point $\mathrm{P}$ and point $\mathrm{Q}$, along the central axis of the hub, is the hub center of mass (C) (not shown in Fig. 3.1). The position vector connecting the hub center of mass and the teeter pin, $\boldsymbol{r}^{P C}$, is:

$$
\boldsymbol{r}^{P C}=-R_{U M} \boldsymbol{g}_{1}
$$

Similar to the tower, the root of each rotor blade can be considered rigid to some radius $R_{H}$ representing the robustness of the hub (hub radius). The length of the flexible part of each blade is thus $R-R_{H}$, where $R$ is the total radius of the rotor (also not shown in Fig. 3.1). The flexible part of each blade is assumed to deflect in the local flapwise (out-of-plane of rotor if pitch and

calculations associated with the kinematics and kinetics of the equations of motion. FAST_AD code developers should consider revising the code to thoroughly implement the addition of this new parameter. 
twist distribution equal zero) and local edgewise (in-plane of rotor if pitch and twist distribution equal zero) directions independently. Local means that the flapwise and edgewise directions are unique to each blade element as defined by the sum of the distributed structural pretwist angle, $\theta_{S}(r)^{9}$, and the blade collective pitch angle. Unlike the tower, the natural mode shapes in each direction are permitted to be different. The natural mode shapes for each blade are assumed to be identical.

Because each blade can have some distributed structural pretwist, defining the deflection in two directions is complicated. The most viable method is to define the total blade curvature as the combination of the local curvature in each local blade element direction (flapwise or edgewise), resolved into in-plane and out-of-plane components by orienting them with the structural pretwist and blade collective pitch angles. This curvature can then be integrated twice to get the total deflection shape. Assuming that the blade deflections are small, the local curvatures in the flapwise and edgewise directions at a span of $r$, and time $t, \kappa_{F}(r, t)$ and $\kappa_{E}(r, t)$ respectively, for blade 1 , are ${ }^{11}$ :

$$
\begin{gathered}
\kappa_{F}(r, t)=q_{1} \frac{d^{2} \phi_{I B F}(r)}{d r^{2}}+q_{11} \frac{d^{2} \phi_{2 B F}(r)}{d r^{2}} \\
\text { and } \\
\kappa_{E}(r, t)=q_{13} \frac{d^{2} \phi_{I B E}(r)}{d r^{2}}
\end{gathered}
$$

where $\phi_{I B F}(r)$ and $\phi_{2 B F}(r)$ are the first and second natural mode shapes, respectively, of the blades in the flapwise direction and $\phi_{I B E}(r)$ is first natural mode shape of the blades in the edgewise direction. The dependency on these curvatures with time is inherent in the generalized coordinates $q_{1}, q_{11}$, and $q_{13}$. In these expressions, the radius along the flexible part of the blade,

${ }^{11}$ The curvature of a curve $\mathrm{y}(\mathrm{x}), \boldsymbol{\kappa}(x)$, is:

$$
\kappa(x)=\frac{\left|\frac{d^{2} y(x)}{d x^{2}}\right|}{\left\{1+\left[\frac{d y(x)}{d x}\right]^{2}\right\}^{3 / 2}}
$$

If the curve is composed only of small deflections, then:

$$
\frac{d y(x)}{d x}<<1
$$

and the curvature simplifies to the following form:

$$
\kappa(x)=\left|\frac{d^{2} y(x)}{d x^{2}}\right|
$$


$r$, ranges from zero to $R-R_{H}$. Note that $r$ equals zero at a span of $R_{H}$ relative to the axis of the hub. The derivation of these natural mode shapes of the blades is presented in section 3.2.

The curvatures in the out-of-plane and in-plane directions at a span of $r$, and time $t, \kappa_{O}(r, t)$ and $\kappa_{I}(r, t)$ respectively, for blade 1 , are ${ }^{12}$ :

$$
\begin{aligned}
& \kappa_{O}(r, t)= {\left[q_{1} \frac{d^{2} \phi_{1 B F}(r)}{d r^{2}}+q_{11} \frac{d^{2} \phi_{2 B F}(r)}{d r^{2}}\right] \cos \left[\theta_{S}(r)+\theta_{P}\right]+} \\
& {\left[q_{13} \frac{d^{2} \phi_{1 B E}(r)}{d r^{2}}\right] \sin \left[\theta_{S}(r)+\theta_{P}\right] } \\
& \text { and } \\
& \kappa_{I}(r, t)=-\left[q_{1} \frac{d^{2} \phi_{1 B F}(r)}{d r^{2}}+q_{11} \frac{d^{2} \phi_{2 B F}(r)}{d r^{2}}\right] \sin \left[\theta_{S}(r)+\theta_{P}\right]+ \\
& {\left[q_{13} \frac{d^{2} \phi_{1 B E}(r)}{d r^{2}}\right] \cos \left[\theta_{S}(r)+\theta_{P}\right] }
\end{aligned}
$$

or equivalently:

$$
\begin{aligned}
& \kappa_{O}(r, t)=q_{1} \frac{d^{2} \phi_{1}(r)}{d r^{2}}+q_{11} \frac{d^{2} \phi_{2}(r)}{d r^{2}}+q_{13} \frac{d^{2} \phi_{3}(r)}{d r^{2}} \\
& \text { and } \\
& \kappa_{I}(r, t)=q_{1} \frac{d^{2} \psi_{1}(r)}{d r^{2}}+q_{11} \frac{d^{2} \psi_{2}(r)}{d r^{2}}+q_{13} \frac{d^{2} \psi_{3}(r)}{d r^{2}}
\end{aligned}
$$

where the twisted shape functions [the $\phi_{i}(r)$ 's and $\psi_{i}(r)$ 's for $i=1,2$, and 3] are defined as:

$$
\frac{d^{2} \phi_{1}(r)}{d r^{2}}=\left[\frac{d^{2} \phi_{1 B F}(r)}{d r^{2}}\right] \cos \left[\theta_{S}(r)+\theta_{P}\right]
$$

\footnotetext{
${ }^{12}$ Since the distributed structural pretwist angle, $\theta_{S}(r)$, is defined in the opposite sense as Wilson et al. (1999), the signs of these equations are different. This, however, is not the only significant difference between the in-plane and out-of-plane curvature equations presented here and the equations presented in Wilson et al. and implemented in the FAST_AD design code. The in-plane and out-of-plane curvatures and corresponding twisted shape functions developed in FAST_AD as indicated by Wilson et al. leave out the blade collective pitch angle from these equations! Thus, when modeling a wind turbine, FAST_AD in essence regards the stiffness orientation of the blades to be fixed when the blades pitch, which physically is not the case. FAST_AD code developers should consider revising the code to correct for this significant error!
} 


$$
\begin{aligned}
& \frac{d^{2} \phi_{2}(r)}{d r^{2}}=\left[\frac{d^{2} \phi_{2 B F}(r)}{d r^{2}}\right] \cos \left[\theta_{S}(r)+\theta_{P}\right] \\
& \frac{d^{2} \phi_{3}(r)}{d r^{2}}=\left[\frac{d^{2} \phi_{I B E}(r)}{d r^{2}}\right] \sin \left[\theta_{S}(r)+\theta_{P}\right] \\
& \frac{d^{2} \psi_{l}(r)}{d r^{2}}=-\left[\frac{d^{2} \phi_{I B F}(r)}{d r^{2}}\right] \sin \left[\theta_{S}(r)+\theta_{P}\right] \\
& \frac{d^{2} \psi_{2}(r)}{d r^{2}}=-\left[\frac{d^{2} \phi_{2 B F}(r)}{d r^{2}}\right] \sin \left[\theta_{S}(r)+\theta_{P}\right] \\
& \frac{d^{2} \psi_{3}(r)}{d r^{2}}=\left[\frac{d^{2} \phi_{1 B E}(r)}{d r^{2}}\right] \operatorname{and}\left[\theta_{S}(r)+\theta_{P}\right]
\end{aligned}
$$

The curvatures can be integrated over $r$ to obtain the deflections of blade 1 in the out-of-plane $\left(\boldsymbol{i}_{1}\right)$ and in-plane $\left(\boldsymbol{i}_{2}\right)$ directions at a span of $r$, and time $t, u(r, t)$ and $v(r, t)$ respectively. Since the deflections at the root of each blade (a span $R_{H}$ relative to the hub axis or $r=0$ ) must be zero, the deflections of blade 1 in the out-of-plane and in-plane directions become:

$$
\begin{gathered}
u(r, t)=\int_{0}^{R-R_{H}}\left[\int_{0}^{r} \kappa_{O}\left(r^{\prime}, t\right) d r^{\prime}\right] d r \\
\text { and } \\
v(r, t)=\int_{0}^{R-R_{H}}\left[\int_{0}^{r} \kappa_{I}\left(r^{\prime}, t\right) d r^{\prime}\right] d r
\end{gathered}
$$

or equivalently:

$$
\begin{gathered}
u(r, t)=q_{1} \phi_{1}(r)+q_{11} \phi_{2}(r)+q_{13} \phi_{3}(r) \\
\text { and } \\
v(r, t)=q_{1} \psi_{1}(r)+q_{11} \psi_{2}(r)+q_{13} \psi_{3}(r)
\end{gathered}
$$

where $r$ ' is a dummy variable representing the span along the flexible part of the blade.

As discussed in section 3.2, an axial (radial or span-wise) deflection of the blades will directly result from any lateral deflection if the flexible blades are assumed to remain fixed in length. By 
an extension of the derivations of section 3.2, this axial deflection for blade 1 at a span of $r$, and time $t, w(r, t)$, is:

$$
w(r, t)=\frac{1}{2} \int_{0}^{r}\left\{\left[\frac{\partial u\left(r^{\prime}, t\right)}{\partial r^{\prime}}\right]^{2}+\left[\frac{\partial v\left(r^{\prime}, t\right)}{\partial r^{\prime}}\right]^{2}\right\} d r^{\prime}
$$

A positive axial deflection is directed along the negative $\boldsymbol{i}_{3}$-axis.

Substituting Eqs. (3.24) and (3.25) into Eq. (3.26), results in:

$$
w(r, t)=\frac{1}{2}\left(q_{1}^{2} S_{11}+q_{11}^{2} S_{22}+q_{13}^{2} S_{33}+2 q_{1} q_{11} S_{12}+2 q_{11} q_{13} S_{23}+2 q_{1} q_{13} S_{13}\right)
$$

where the symmetric, three by three, matrix $[S]$ is:

$$
S_{i j}=\int_{0}^{r}\left\{\left[\frac{d \phi_{i}\left(r^{\prime}\right)}{d r^{\prime}}\right]\left[\frac{d \phi_{j}\left(r^{\prime}\right)}{d r^{\prime}}\right]+\left[\frac{d \psi_{i}\left(r^{\prime}\right)}{d r^{\prime}}\right]\left[\frac{d \psi_{j}\left(r^{\prime}\right)}{d r^{\prime}}\right]\right\} d r^{\prime}
$$

The position vector connecting any point $\mathrm{S}$ on the deflected blade 1 to the blade axes intersection point $(\mathrm{O}), \boldsymbol{r}^{Q S}$, is:

$$
\boldsymbol{r}^{Q S}=u(r, t) \boldsymbol{i}_{1}+v(r, t) \boldsymbol{i}_{2}+\left[r+R_{H}-w(r, t)\right] \boldsymbol{i}_{3}
$$

The position vector connecting any point $S^{\prime}$ on the undeflected blade 1 to the blade axis intersection point $(\mathrm{O}), \boldsymbol{r}^{Q S^{\prime}}$, is:

$$
\boldsymbol{r}^{Q S^{\prime}}=\left(r+R_{H}\right) \boldsymbol{i}_{3}
$$

For blade 2, only $q_{2}$ needs to be substituted in for $q_{1}, q_{12}$ in for $q_{11}$, and $q_{14}$ in for $q_{13}$. The twisted shape functions [the $\phi_{i}(r)$ 's and $\psi_{i}(r)$ 's for $i=1,2$, and 3] are not dependent on the blade considered.

Fixed quantities (not dependent on time) can be translated to any coordinate system by simple coordinate transformations:

From tower top to inertial:

$$
\left\{\begin{array}{l}
\boldsymbol{a}_{1} \\
\boldsymbol{a}_{2} \\
\boldsymbol{a}_{3}
\end{array}\right\}=\left[\begin{array}{ccc}
\cos \left(\theta_{7}\right) & -\sin \left(\theta_{7}\right) & 0 \\
\sin \left(\theta_{7}\right) \cos \left(\theta_{8}\right) & \cos \left(\theta_{7}\right) \cos \left(\theta_{8}\right) & -\sin \left(\theta_{8}\right) \\
\sin \left(\theta_{7}\right) \sin \left(\theta_{8}\right) & \cos \left(\theta_{7}\right) \sin \left(\theta_{8}\right) & \cos \left(\theta_{8}\right)
\end{array}\right]\left\{\begin{array}{l}
\boldsymbol{b}_{1} \\
\boldsymbol{b}_{2} \\
\boldsymbol{b}_{3}
\end{array}\right\}
$$

From yaw to tower top: 


$$
\left\{\begin{array}{l}
\boldsymbol{b}_{1} \\
\boldsymbol{b}_{2} \\
\boldsymbol{b}_{3}
\end{array}\right\}=\left[\begin{array}{ccc}
\cos \left(q_{6}\right) & 0 & \sin \left(q_{6}\right) \\
0 & 1 & 0 \\
-\sin \left(q_{6}\right) & 0 & \cos \left(q_{6}\right)
\end{array}\right]\left\{\begin{array}{l}
\boldsymbol{d}_{1} \\
\boldsymbol{d}_{2} \\
\boldsymbol{d}_{3}
\end{array}\right\}
$$

From tilt to yaw:

$$
\left\{\begin{array}{l}
\boldsymbol{d}_{\boldsymbol{I}} \\
\boldsymbol{d}_{2} \\
\boldsymbol{d}_{3}
\end{array}\right\}=\left[\begin{array}{ccc}
\cos \left(q_{5}\right) & -\sin \left(q_{5}\right) & 0 \\
\sin \left(q_{5}\right) & \cos \left(q_{5}\right) & 0 \\
0 & 0 & 1
\end{array}\right]\left\{\begin{array}{l}
\boldsymbol{c}_{1} \\
\boldsymbol{c}_{2} \\
\boldsymbol{c}_{3}
\end{array}\right\}
$$

From azimuth to tilt:

$$
\left\{\begin{array}{l}
\boldsymbol{c}_{1} \\
\boldsymbol{c}_{2} \\
\boldsymbol{c}_{3}
\end{array}\right\}=\left[\begin{array}{ccc}
1 & 0 & 0 \\
0 & \cos \left[q_{4}+z(4)\right] & -\sin \left[q_{4}+z(4)\right] \\
0 & \sin \left[q_{4}+z(4)\right] & \cos \left[q_{4}+z(4)\right]
\end{array}\right]\left\{\begin{array}{l}
\boldsymbol{e}_{1} \\
\boldsymbol{e}_{2} \\
\boldsymbol{e}_{3}
\end{array}\right\}
$$

From teeter to azimuth:

$$
\left\{\begin{array}{l}
\boldsymbol{e}_{1} \\
\boldsymbol{e}_{2} \\
\boldsymbol{e}_{3}
\end{array}\right\}=\left[\begin{array}{ccc}
\cos \left(q_{3}\right) & 0 & \sin \left(q_{3}\right) \\
0 & 1 & 0 \\
-\sin \left(q_{3}\right) & 0 & \cos \left(q_{3}\right)
\end{array}\right]\left\{\begin{array}{l}
\boldsymbol{f}_{1} \\
\boldsymbol{f}_{2} \\
\boldsymbol{f}_{3}
\end{array}\right\}
$$

From delta-3 to teeter:

$$
\left\{\begin{array}{l}
\boldsymbol{f}_{1} \\
\boldsymbol{f}_{2} \\
\boldsymbol{f}_{3}
\end{array}\right\}=\left[\begin{array}{ccc}
1 & 0 & 0 \\
0 & \cos \left(\delta_{3}\right) & -\sin \left(\delta_{3}\right) \\
0 & \sin \left(\delta_{3}\right) & \cos \left(\delta_{3}\right)
\end{array}\right]\left\{\begin{array}{l}
\boldsymbol{g}_{1} \\
\boldsymbol{g}_{2} \\
\boldsymbol{g}_{3}
\end{array}\right\}
$$

From coning to delta-3:

$$
\left\{\begin{array}{l}
\boldsymbol{g}_{1} \\
\boldsymbol{g}_{2} \\
\boldsymbol{g}_{3}
\end{array}\right\}=\left[\begin{array}{ccc}
\cos (\beta) & 0 & \sin (\beta) \\
0 & 1 & 0 \\
-\sin (\beta) & 0 & \cos (\beta)
\end{array}\right]\left\{\begin{array}{l}
\boldsymbol{i}_{1} \\
\boldsymbol{i}_{2} \\
\boldsymbol{i}_{3}
\end{array}\right\}
$$

Equation (3.37) is applicable to either blade. When representing blade $1, \beta$ equals $\beta_{1}$ and the reference frame $\boldsymbol{i}$ is specific to blade 1. When representing blade 2, $\beta$ equals $\beta_{2}$ and the reference frame $\boldsymbol{i}$ is specific to blade 2 . 


\subsection{Blade and Tower Deflections}

The structural model of FAST_AD considers the blades and tower to be flexible cantilevered beams with continuously distributed mass and stiffness. In theory, such bodies possess an infinite number of DOFs, since an infinite number of coordinates are needed to specify the position of every point on the body. In practice, such bodies are modeled as a linear sum of known shapes of the dominant normal vibration modes. This technique is known as the normal mode summation method and reduces the number of DOFs from infinity to $N$, the number of normal modes considered to be dominant. With this method, the lateral deflection (perpendicular to the undeformed beam) anywhere on the flexible beam at any time, $u(z, t)$, is given as the summation of the products of each normal mode shape, $\phi_{a}(z)$, and their associated generalized coordinate, $q_{a}(t)$ :

$$
u(z, t)=\sum_{a=1}^{N} \phi_{a}(z) q_{a}(t)
$$

The normal mode shape for mode $a, \phi_{a}(z)$, is purely a function of the distance $z$ along the beam $(\mathrm{z}=0$ at the fixed end and $\mathrm{z}=\mathrm{Z}$ at the free end) and the generalized coordinate associated with normal mode $a, q_{a}(t)$, is purely a function of time $t$. Each normal mode has an associated natural frequency, $\omega_{a}$, and phase, $\psi_{a}$. The generalized coordinate associated with a normal mode is customarily allowed to be the deflection of the free end of the cantilever beam; thus, each normal mode shape is dimensionless and normalized so it is equal to unity at the free end.

When each normal mode shape is known, $N$ parameters are required to specify the deflection of the flexible body at any time. Thus, alternatively, the lateral deflection of the flexible body could be expressed using $N$ other functions, $\varphi_{b}(z)$, not unique to each normal mode:

$$
u(z, t)=\sum_{b=p}^{N+p-1} \varphi_{b}(z) c_{b}(t)
$$

where $c_{b}(t)$ is the generalized coordinate associated with the function $\varphi_{b}(z)$. The $\varphi_{b}(z)$ 's are known as shape functions and the parameter $p$ is chosen for convenience.

Since the shape functions are not unique to each normal mode, meaning that each normal mode is related to all shape functions, there is a relationship such that the normal mode shapes form a linear combination of the shape functions:

$$
\phi_{a}(z)=\sum_{b=p}^{N+p-1} C_{a, b} \varphi_{b}(z) \quad(a=1,2, \ldots, N)
$$

where $C_{a, b}$ is the constant proportionality coefficient associated with the $b^{\text {th }}$ shape function and the $a^{\text {th }}$ normal mode. This is known as the Rayleigh-Ritz method.

In FAST_AD, each normal mode shape is assumed to be expressible as a polynomial; thus, the $b^{\text {th }}$ shape function is defined as: 


$$
\varphi_{b}(z)=\left(\frac{z}{Z}\right)^{b}
$$

Since the slope of a cantilevered beam must be zero at the fixed end, $p$ must be no smaller than two if the shape functions are to accurately represent the normal mode shapes. Thus, the FAST_AD design code requires that $p$ equal two. FAST_AD allows $N$ to be as high as five. In FAST_AD, the constant proportionality coefficients associated with each shape function and normal mode are parameters requested in the input file. A preprocessor code entitled Modes enables users of FAST_AD to obtain these parameters. The theory employed by Modes is also developed here.

Using Lagrange's equations for a conservative, scleronomic system ${ }^{13}$, the equations of motion for an $N$-DOF system are equivalent to:

$$
\sum_{j=p}^{N+p-1} m_{i j} \ddot{c}_{j}(t)+\sum_{j=p}^{N+p-1} k_{i j} c_{j}(t)=0 \quad(i=p, p+1, \ldots, N+p-1)
$$

where the generalized mass and stiffness, $m_{i j}$ and $k_{i j}$ respectively, are defined in terms of the kinetic energy, $T$, and potential energy, $V$ :

$$
\begin{aligned}
& T=\frac{1}{2} \sum_{i=p}^{N+p-l} \sum_{j=p}^{N+p-1} m_{i j} \dot{c}_{i}(t) \dot{c}_{j}(t) \\
& \text { and } \\
& V=\frac{1}{2} \sum_{i=p}^{N+p-1} \sum_{j=p}^{N+p-1} k_{i j} c_{i}(t) c_{j}(t)
\end{aligned}
$$

Now, when the flexible beam is vibrating at a specific natural mode, say $a=m$, the following conditions result:

\footnotetext{
${ }^{13}$ Lagrange's equations are the scalar equivalents of Newton's law of motion:

$$
\boldsymbol{F}=m \boldsymbol{a}
$$
}

Lagrange's equations for a conservative, scleronomic system can be stated in terms of the standard form of Lagrange's equations:

$$
\frac{d}{d t}\left(\frac{\partial L}{\partial \dot{q}_{i}}\right)-\frac{\partial L}{\partial q_{i}}=0 \quad(i=1,2, \ldots, N)
$$

where the Lagrangian function, $L$, is defined as:

$$
L=T-V
$$




$$
\begin{gathered}
q_{a}(t)= \begin{cases}Q_{a} \sin \left(\omega_{a} t+\psi_{a}\right) & \text { for } a=m \\
0 & \text { otherwise }\end{cases} \\
\text { and } \\
c_{b}(t)=C_{m, b} q_{m}(t) \quad(b=p, p+1, \ldots, N+p-1)
\end{gathered}
$$

where $Q_{a}$ is the amplitude of the deflection of the tip of the flexible beam associated with natural mode $a$.

Substituting Eq. (3.45) into Eq. (3.46), then substituting the resulting equation into Eq. (3.42), results in (the subscript has been dropped from the specific natural mode):

$$
\sum_{j=p}^{N+p-1}\left(-\omega^{2} m_{i j}+k_{i j}\right) C_{j}=0 \quad(i=p, p+1, \ldots, N+p-1)
$$

Equation (3.47) can be written in matrix form:

$$
\left(-\omega^{2}[M]+[K]\right)\{C\}=\{0\}
$$

where the generalized mass matrix, $[M]$, and generalized stiffness matrix, $[K]$, are both $N \times N$ matrices and the coefficient vector, $\{C\}$, is an $N \times 1$ vector. The determinant of the matrix premultiplying the coefficient vector results in an $N^{\text {th }}$-degree algebraic equation in $\omega^{2}$, which is called the characteristic equation. The $N$ roots, $\omega_{a}^{2}$, are the eigenvalues, each being the square of the natural frequency associated with normal mode $a$. The eigenvector associated with each eigenvalue, $\{C\}_{a}$, defines the constant proportionality coefficients associated with normal mode $a$ (the $C_{a, b}$ 's).

In FAST_AD and Modes, the tower is modeled as an inverted cantilever beam with a point mass affixed to its free end. The point mass, $M_{T o p}$, represents the combined mass of the base plate, nacelle, hub, and blades. The tower is assumed to deflect in the longitudinal and lateral directions independently. The stiffness distributions in each direction are assumed to be identical; consequently, the associated natural mode shapes and frequencies are assumed to be identical in each direction.

The kinetic energy of the tower has a component associated with the distributed mass of the beam and a component associated with the point mass:

$$
T=T_{\text {Beam }}+T_{\text {Top }}
$$

The kinetic energy of a beam as developed in Thomson and Dahleh (1998) is: 


$$
T_{\text {Beam }}=\frac{1}{2} \sum_{i=p}^{N+p-l} \sum_{j=p}^{N+p-1}\left(\int_{0}^{H} \mu_{T}(h) \varphi_{i}(h) \varphi_{j}(h) d h\right) \dot{c}_{i}(t) \dot{c}_{j}(t)
$$

where $\mu_{T}(h)$ is the distributed lineal density of the beam (tower).

The kinetic energy of the affixed point mass is:

$$
T_{T o p}=\frac{1}{2} \sum_{i=p}^{N+p-l} \sum_{j=p}^{N+p-t}\left(M_{T o p} \varphi_{i}(H) \varphi_{j}(H)\right) \dot{c}_{i}(t) \dot{c}_{j}(t)
$$

Since each shape function equals unity at the free end [see Eq. (3.41)], the kinetic energy of the affixed point mass can be simplified:

$$
T_{\text {Top }}=\frac{1}{2} \sum_{i=p}^{N+p-l} \sum_{j=p}^{N+p-1}\left(M_{\text {Top }}\right) \dot{c}_{i}(t) \dot{c}_{j}(t)
$$

Equations (3.43), (3.49), (3.50), and (3.52) show that the generalized mass of the tower is:

$$
m_{i j}=M_{T o p}+\int_{0}^{H} \mu_{T}(h) \varphi_{i}(h) \varphi_{j}(h) d h
$$

The potential energy of the tower has a component associated with the distributed stiffness of the beam and a component associated with gravity:

$$
V=V_{\text {Beam }}+V_{\text {Gravity }}
$$

The potential energy of a beam as developed in Thomson and Dahleh (1998) is:

$$
V_{\text {Beam }}=\frac{1}{2} \sum_{i=p}^{N+p-l} \sum_{j=p}^{N+p-1}\left(\int_{0}^{H} E I_{T}(h) \frac{d^{2} \varphi_{i}(h)}{d h^{2}} \frac{d^{2} \varphi_{j}(h)}{d h^{2}} d h\right) c_{i}(t) c_{j}(t)
$$

where $E I_{T}(h)$ is the distributed stiffness of the beam (tower).

Gravity tends to reduce the stiffness of the tower. The potential energy caused by gravity of an inverted beam with a point mass affixed to its free end is the product of the gravitational force and the distance upon which this force acts (the negative sign promotes the notion that gravity reduces the stiffness):

$$
V_{\text {Gravity }}=-g\left[M_{\text {Top }} v(H, t)+\int_{0}^{H} \mu_{T}(h) v(h, t) d h\right]
$$

where $v(h, t)$ is the axial deflection of the flexible cantilever beam at time $t$ and an original elevation $h$. The axial deflection is not due to compression of the tower. Instead, the axial 
deflection is a combined result of assuming the flexible beam remains fixed in length (measured along the beam's central axis) and the fact that the free end of a cantilevered beam must move closer to the fixed end when the beam deflects laterally. That is, the axial deflection is directly and interdependently related to the lateral deflection. This relationship can be obtained by examining the geometry of the deflection(s) as depicted graphically in Fig. 3.2.

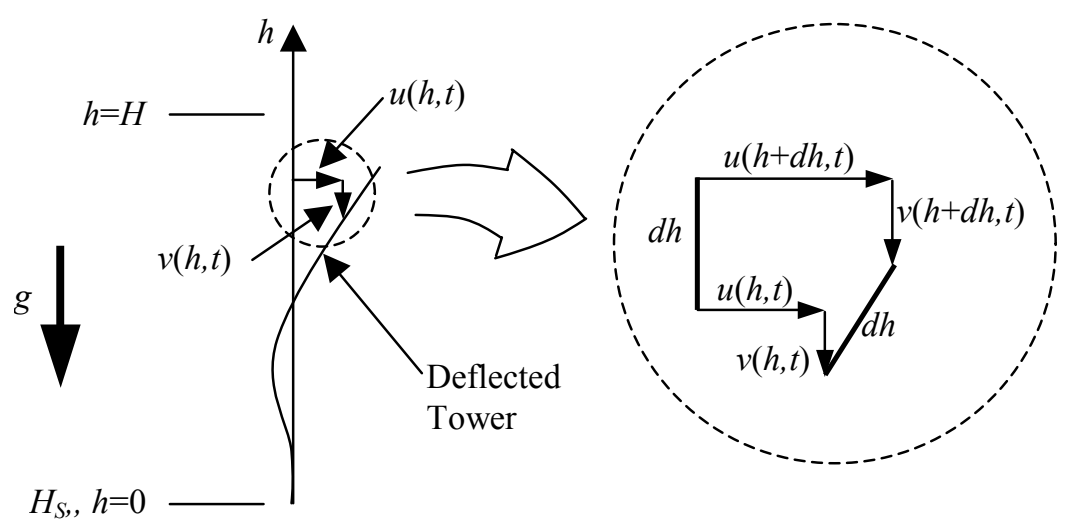

Figure 3.2: Tower deflection geometry

Expanding the lateral and axial deflections about any elevation $h$ using a first order Taylor series approximation results in:

$$
\begin{gathered}
u(h+d h, t)=u(h, t)+\frac{\partial u(h, t)}{\partial h} d h \\
\text { and } \\
v(h+d h, t)=v(h, t)+\frac{\partial v(h, t)}{\partial h} d h
\end{gathered}
$$

The Pythagorean theorem can be applied to the geometry of the deflection of a tower element (see the dashed circle magnification of Fig. 3.2) to easily obtain:

$$
\left[\frac{\partial u(h, t)}{\partial h}\right]^{2}+\left[\frac{\partial v(h, t)}{\partial h}\right]^{2}=2 \frac{\partial v(h, t)}{\partial h}
$$

Equation (3.59) can be solved for the gradient of the axial deflection of the tower using the quadratic formula. This action results in:

$$
\frac{\partial v(h, t)}{\partial h}=1 \pm \sqrt{1-\left[\frac{\partial u(h, t)}{\partial h}\right]^{2}}
$$

The lateral deflection of the tower is assumed to be small so that: 


$$
\frac{\partial u(h, t)}{\partial h}<<1
$$

Applying Eq. (3.61) to Eq. (3.60) results in two simplified solutions to the gradient of the axial deflection of the tower:

$$
\frac{\partial v(h, t)}{\partial h}=2-\frac{1}{2}\left[\frac{\partial u(h, t)}{\partial h}\right]^{2} \text { and } \frac{1}{2}\left[\frac{\partial u(h, t)}{\partial h}\right]^{2}
$$

However, if the lateral deflection of the tower is small so must the axial deflection:

$$
\frac{\partial v(h, t)}{\partial h}<<1
$$

Consequently, the only valid solution is the latter:

$$
\frac{\partial v(h, t)}{\partial h}=\frac{1}{2}\left[\frac{\partial u(h, t)}{\partial h}\right]^{2}
$$

This expression can be integrated over $h$ to obtain the axial deflection of the tower as a function of the tower's lateral deflection. Since the slope of a tower must be zero at the fixed end (an elevation $H_{S}$ above the surface of the Earth or $h=0$ ), the axial deflection of the tower becomes:

$$
v(h, t)=\frac{1}{2} \int_{0}^{h}\left[\frac{\partial u\left(h^{\prime}, t\right)}{\partial h^{\prime}}\right]^{2} d h^{\prime}
$$

where $h$ ' is a dummy variable representing the elevation along the flexible part of the tower. Employing the shape functions through the use of Eq. (3.39), Eq. (3.65) can be rewritten:

$$
v(h, t)=\frac{1}{2} \sum_{i=p}^{N+p-l} \sum_{j=p}^{N+p-l}\left(\int_{0}^{h} \frac{d \varphi_{i}\left(h^{\prime}\right)}{d h^{\prime}} \frac{d \varphi_{j}\left(h^{\prime}\right)}{d h^{\prime}} d h^{\prime}\right) c_{i}(t) c_{j}(t)
$$

Substituting Eq. (3.66) into Eq. (3.56) results in the following equation for the potential energy of the tower caused by gravity:

$$
\begin{aligned}
V_{\text {Gravity }}= & -g \frac{1}{2} \sum_{i=p}^{N+p-1} \sum_{j=p}^{N+p-1}\left[M_{\text {Top }} \int_{0}^{H} \frac{d \varphi_{i}(h)}{d h} \frac{d \varphi_{j}(h)}{d h} d h+\right. \\
& \left.\int_{0}^{H} \mu_{T}(h)\left(\int_{0}^{h} \frac{d \varphi_{i}\left(h^{\prime}\right)}{d h^{\prime}} \frac{d \varphi_{j}\left(h^{\prime}\right)}{d h^{\prime}} d h^{\prime}\right) d h\right] c_{i}(t) c_{j}(t)
\end{aligned}
$$

Equations (3.44), (3.54), (3.55), and (3.67) show that the generalized stiffness of the tower is: 


$$
\begin{aligned}
k_{i j}= & \int_{0}^{H} E I_{T}(h) \frac{d^{2} \varphi_{i}(h)}{d h^{2}} \frac{d^{2} \varphi_{j}(h)}{d h^{2}} d h- \\
& g\left[M_{T o p} \int_{0}^{H} \frac{d \varphi_{i}(h)}{d h} \frac{d \varphi_{j}(h)}{d h} d h+\int_{0}^{H} \mu_{T}(h)\left(\int_{0}^{h} \frac{d \varphi_{i}\left(h^{\prime}\right)}{d h^{\prime}} \frac{d \varphi_{j}\left(h^{\prime}\right)}{d h^{\prime}} d h^{\prime}\right) d h\right]^{(3.6}
\end{aligned}
$$

After integrating by parts and simplifying, Eq. (3.68) is equivalent to ${ }^{14}$ :

$$
\begin{aligned}
k_{i j}= & \int_{0}^{H} E I_{T}(h) \frac{d^{2} \varphi_{i}(h)}{d h^{2}} \frac{d^{2} \varphi_{j}(h)}{d h^{2}} d h- \\
& g \int_{0}^{H}\left[M_{T o p}+\int_{h}^{H} \mu_{T}\left(h^{\prime}\right) d h^{\prime}\right] \frac{d \varphi_{i}(h)}{d h} \frac{d \varphi_{j}(h)}{d h} d h
\end{aligned}
$$

In FAST_AD and Modes, each blade is modeled as a rotating cantilever beam with a point mass affixed to its free end. This time labeled $M_{\text {Tip }}$, the point mass represents the mass of the tip brake if applicable. In the calculation of the generalized mass and generalized stiffness, the cantilever beam is assumed to rotate with an angular speed, $\Omega$, about an axis perpendicular to the axis of the beam, which is equivalent to assuming that there is no coning angle, $\beta$, associated with each blade ${ }^{15}$ and no teeter motion. The flexible part of each blade is assumed to deflect in the flapwise (out-of-plane of rotor if pitch and twist distribution equal zero) and edgewise (in-plane of rotor if pitch and twist distribution equal zero) directions independently. Unlike the tower, the stiffness distributions in each direction are permitted to be different.

The kinetic energy of the blades in the reference frame rotating with the rotor is identical in form to the kinetic energy of the tower [reference Eq. (3.49), Eq. (3.50), and Eq. (3.52)] since the rotation of the blades contributes nothing to the kinetic energy in this reference frame. Thus, the generalized mass of each blade can be written in a form similar to Eq. (3.53):

\footnotetext{
${ }^{14}$ This is in contrast to the generalized stiffness of the tower used by FAST_AD and program Modes specified as:

$$
k_{i j}=\int_{0}^{H} E I_{T}(h) \frac{d^{2} \varphi_{i}(h)}{d h^{2}} \frac{d^{2} \varphi_{j}(h)}{d h^{2}} d h-g \int_{0}^{H}\left[M_{T o p}+(H-h) \mu_{T}(h)\right] \frac{d \varphi_{i}(h)}{d h} \frac{d \varphi_{j}(h)}{d h} d h
$$

as indicated by Wilson et al. (1999). The equation for the generalized stiffness of the tower as implemented in Modes preprocessor code and FAST_AD is valid only if the distributed lineal density of the tower is constant with elevation (i.e., not dependent on the elevation $h$ along the flexible portion of the tower). For single section tubular towers, this assumption is valid. However, if the distributed lineal density of the tower is not constant with elevation, than the mode shapes outputted by Modes and the generalized stiffness employed by FAST_AD will be in error. This error is more substantial when the distributed lineal density has greater variability. Code developers of FAST_AD and Modes should consider revising the code to correct for this error!
}

\footnotetext{
${ }^{15}$ For turbines with small coning angles $(\beta<<1$ radians), this assumption begets negligible errors. However, for turbines with relatively large coning angles, the errors brought about by this assumption may be significant. Code developers of FAST_AD and Modes should consider enhancing the applicability of the codes by incorporating $a$ coning angle into the computation of the generalized mass and stiffness of the blades.
} 


$$
m_{i j}=M_{T i p}+\int_{0}^{R-R_{H}} \mu_{B}(r) \varphi_{i}(r) \varphi_{j}(r) d r
$$

where $\mu_{B}(r)$ is the distributed lineal density of the beam (blades). Equation (3.70) is valid for both the flapwise and edgewise directions.

Neglecting gravity, the potential energy of each blade has a component associated with the distributed stiffness of the beam and a component associated with centrifugal stiffening as a result of rotor rotation:

$$
V=V_{\text {Beam }}+V_{\text {Rotation }}
$$

The potential energy component of each blade associated with the distributed stiffness of the beam is identical in form to that of Eq. (3.55). The potential energy caused by rotor rotation is:

$$
V_{\text {Rotation }}=\Omega^{2}\left[M_{\text {Tip }} R v\left(R-R_{H}, t\right)+\int_{0}^{R-R_{H}} \mu_{B}(r)\left(R_{H}+r\right) v(r, t) d r\right]
$$

The centrifugal forces tend to increase the stiffness of each blade.

The potential energy caused by rotor rotation is essentially the product of the centrifugal forces and the distances upon which they act. For example, the centrifugal force acting on a blade's tip brake as used in Eq. (3.72) is $\Omega^{2} M_{T i p} R$, and the distance upon which this force acts is $v\left(R-R_{H}, t\right)$. This expression assumes centrifugal forces acting on a blade do not change when the blade deflects. To be technically correct, the centrifugal forces should be regarded as functions of the blade deflection since, for example, the centrifugal force acting on a blade's tip brake would be slightly less than $\Omega^{2} M_{T i p} R$ if the tip was deflected substantially since the distance to the tip from the center of rotation would be shorter than $R$ by a distance $v\left(R-R_{H}, t\right)$. If this correlation between centrifugal force and deflection were accounted for, however, terms involving $v(r, t)^{2}$ would appear in Eq. (3.72). This action would prevent the centrifugal forces from being derivable from a conservative force field and would beget considerable difficulties in the ensuing analysis. Therefore, FAST_AD and Modes assume that the centrifugal forces are not functions of the blade deflection. If the deflections of the blades are assumed to be small (the blades are relatively stiff), this action introduces negligible errors.

As for the tower, the axial (radial or span-wise) deflection of the flexible cantilever beam at time $t$ and an original span $r, v(r, t)$, is a direct result of the assumption that the flexible beam remains fixed in length (measured along the beam's central axis) when deflecting. Thus, assuming small deflections, the axial deflection of each blade is directly related to its lateral deflection [see Eqs. (3.65) and (3.66) for towers]:

$$
v(r, t)=\frac{1}{2} \int_{0}^{r}\left[\frac{\partial u\left(r^{\prime}, t\right)}{\partial r^{\prime}}\right]^{2} d r^{\prime}
$$




$$
v(r, t)=\frac{1}{2} \sum_{i=p}^{N+p-l} \sum_{j=p}^{N+p-l}\left(\int_{0}^{r} \frac{d \varphi_{i}\left(r^{\prime}\right)}{d r^{\prime}} \frac{d \varphi_{j}\left(r^{\prime}\right)}{d r^{\prime}} d r^{\prime}\right) c_{i}(t) c_{j}(t)
$$

where $r$ ' is a dummy variable representing the span along the flexible part of each blade.

Substituting Eq. (3.74) into Eq. (3.72) results in Eq. 3.75 for the potential energy of each blade caused by rotor rotation:

$$
\begin{aligned}
V_{\text {Rotation }}= & \frac{1}{2} \Omega^{2} \sum_{i=p}^{N+p-l} \sum_{j=p}^{N+p-l}\left[M_{T i p} R \int_{0}^{R-R_{H}} \frac{d \varphi_{i}(r)}{d r} \frac{d \varphi_{j}(r)}{d r} d r+\right. \\
& \left.\int_{0}^{R-R_{H}} \mu_{B}(r)\left(R_{H}+r\right)\left(\int_{0}^{r} \frac{d \varphi_{i}\left(r^{\prime}\right)}{d r^{\prime}} \frac{d \varphi_{j}\left(r^{\prime}\right)}{d r^{\prime}} d r^{\prime}\right) d r\right] c_{i}(t) c_{j}(t)
\end{aligned}
$$

Consequently, the generalized stiffness of each blade is:

$$
\begin{aligned}
k_{i j}=\int_{0}^{R-R_{H}} E I_{B}(r) \frac{d^{2} \varphi_{i}(r)}{d r^{2}} \frac{d^{2} \varphi_{j}(r)}{d r^{2}} d r+ \\
\Omega^{2}\left[M_{T i p} R \int_{0}^{R-R_{H}} \frac{d \varphi_{i}(r)}{d r} \frac{d \varphi_{j}(r)}{d r} d r+\right. \\
\left.\quad \int_{0}^{R-R_{H}} \mu_{B}(r)\left(R_{H}+r\right)\left(\int_{0}^{r} \frac{d \varphi_{i}\left(r^{\prime}\right)}{d r^{\prime}} \frac{d \varphi_{j}\left(r^{\prime}\right)}{d r^{\prime}} d r^{\prime}\right) d r\right]
\end{aligned}
$$

where $E I_{B}(r)$ is the distributed stiffness of the beam (blades).

After integrating by parts and simplifying, it can be shown that Eq. (3.76) is equivalent to ${ }^{16}$ :

${ }^{16}$ This is in contrast to the generalized stiffness of each blade as specified by Wilson et al. (1999):

$$
\begin{aligned}
k_{i j}= & \int_{0}^{R-R_{H}} E I_{B}(r) \frac{d^{2} \varphi_{i}(r)}{d r^{2}} \frac{d^{2} \varphi_{j}(r)}{d r^{2}} d r+ \\
& \Omega^{2} \int_{0}^{R-R_{H}}\left[M_{T i p} R+\int_{r}^{R-R_{H}} \mu_{B}\left(r^{\prime}\right)\left(R_{H}+r^{\prime}\right) d r^{\prime}\right] \frac{d \varphi_{i}(r)}{d r} \frac{d \varphi_{j}(r)}{d r} d r+\Omega^{2} m_{i j} \sin (\theta)
\end{aligned}
$$

where $\theta$ equals $\pi / 2$ for edgewise motion and zero for flapwise motion. The only difference between this equation and the corresponding equation in the main body of the thesis is the addition of the term containing $\sin (\theta)$. The term associated with the $\sin (\theta)$ however, is not implemented in the FAST_AD nor the Modes preprocessor code, thus FAST_AD and Modes do compute the generalized stiffness in the flapwise and edgewise directions correctly. Code developers should consider revising this equation in the FAST_AD user's manual. 


$$
\begin{aligned}
k_{i j}= & \int_{0}^{R-R_{H}} E I_{B}(r) \frac{d^{2} \varphi_{i}(r)}{d r^{2}} \frac{d^{2} \varphi_{j}(r)}{d r^{2}} d r+ \\
& \Omega^{2} \int_{0}^{R-R_{H}}\left[M_{T i p} R+\int_{r}^{R-R_{H}} \mu_{B}\left(r^{\prime}\right)\left(R_{H}+r^{\prime}\right) d r^{\prime}\right] \frac{d \varphi_{i}(r)}{d r} \frac{d \varphi_{j}(r)}{d r} d r
\end{aligned}
$$

The distributed stiffness of the flexible part of each blade is generally greater edgewise than flapwise. Otherwise there is essentially no difference in the computation of the generalized stiffness of the blades in the flapwise and edgewise directions, since centrifugal forces are assumed to be unaffected by blade deflection.

\subsection{Kinematics}

The geometry, coordinate systems, and DOFs of a two-bladed HAWT as modeled by FAST_AD and discussed in the previous two sections can be used to develop the kinematics expressions for the entire structure.

Applying the addition theorem for angular velocities ${ }^{17}$ yields the following form of the angular velocity of the hub in the inertial reference frame, ${ }^{E} \omega^{H}$ :

$$
{ }^{E} \omega^{H}={ }^{E} \omega^{B}+{ }^{B} \omega^{N}+{ }^{N} \omega^{L}+{ }^{L} \omega^{H}
$$

where ${ }^{E} \omega^{B}$ is the angular velocity of the tower-top base plate in the inertial reference frame, ${ }^{B} \omega^{N}$ is the angular velocity of the nacelle relative to the tower-top base plate, ${ }^{N} \omega^{L}$ is the angular velocity of the low-speed shaft relative to the nacelle, and ${ }^{L} \omega^{H}$ is the angular velocity of the hub (rotor) relative to the low-speed shaft. The angular velocity of the tower-top base plate in the inertial reference frame, ${ }^{\boldsymbol{E}} \boldsymbol{\omega}^{\boldsymbol{B}}$, is related to the deflection of the tower ${ }^{18}$ :

$$
{ }^{E} \omega^{B}=\dot{\theta}_{8} a_{1}+\dot{\theta}_{7} a_{3}
$$

The angular velocity of the nacelle relative to the tower-top base plate, ${ }^{B} \omega^{N}$, has a component associated with the rate of yaw and the tilt rate:

17 The addition theorem for angular velocities, which asserts that the angular velocity of rigid body $\mathrm{B}$ in reference frame $\mathrm{A}$ can be expressed using $n$ auxiliary reference frame $\mathrm{A}_{1}, \mathrm{~A}_{2}, \ldots, \mathrm{A}_{\mathrm{n}}$, can be stated as:

$$
{ }^{A} \omega^{B}={ }^{A} \omega^{A_{1}}+{ }^{A_{1}} \omega^{A_{2}}+\cdots+{ }^{A_{n-1}} \omega^{A_{n}}+{ }^{A_{n}} \omega^{B}
$$

${ }^{18}$ Wilson et al. (1999) define the angular velocity of the hub in the inertial reference frame:

$$
{ }^{E} \omega^{B}=\dot{\theta}_{8} a_{1}-\dot{\theta}_{7} a_{3}
$$

This definition $i s$ equivalent to the definition in the main body of the report, however, since Wilson et al. (1999) define the time derivative of the longitudinal tower-top rotation without a negative sign [see Eq. (3.4)]. To be consistent with sign conventions and coordinate systems, it is more proper to define the angular velocity of the hub in the inertial reference frame as is done in the main body of the report. 


$$
{ }^{B} \omega^{N}=\dot{q}_{6} d_{2}+\dot{q}_{5} d_{3}
$$

The time derivative of the azimuth angle relates the angular velocity of the low-speed shaft to that of the nacelle:

$$
{ }^{N} \omega^{L}=\dot{q}_{4} e_{1}
$$

Finally, the teeter rate relates the angular velocity of the hub (rotor) to that of the low-speed shaft:

$$
{ }^{L} \omega^{H}=\dot{q}_{3} g_{2}
$$

The previous five equations can be combined to give the following form of the angular velocity of the hub in the inertial reference frame:

$$
{ }^{E} \omega^{H}=\dot{\theta}_{8} a_{1}+\dot{\theta}_{7} a_{3}+\dot{q}_{6} d_{2}+\dot{q}_{5} \boldsymbol{d}_{3}+\dot{q}_{4} \boldsymbol{e}_{1}+\dot{q}_{3} \boldsymbol{g}_{2}
$$

If the velocity of the axial deflection of the tower is assumed to be negligible, the velocity of the tower-top base plate $(\mathrm{O})$ in the inertial reference frame, ${ }^{E} \boldsymbol{v}^{\boldsymbol{O}}$, is ${ }^{19}$ :

$$
{ }^{E} v^{o}=\dot{u}_{7} a_{1}+\dot{u}_{8} a_{3}
$$

The velocity of any other point T on the flexible tower in the inertial frame, ${ }^{\boldsymbol{E}} \boldsymbol{v}^{\boldsymbol{T}}$, is:

$$
{ }^{\boldsymbol{E}} \boldsymbol{v}^{\boldsymbol{T}}=\left[\dot{q}_{7} \phi_{1 T}(h)+\dot{q}_{9} \phi_{2 T}(h)\right] \boldsymbol{a}_{1}+\left[\dot{q}_{8} \phi_{I T}(h)+\dot{q}_{10} \phi_{2 T}(h)\right] \boldsymbol{a}_{3}
$$

where $h$ is the elevation of point T along the flexible part of the tower and ranges from zero to $H$. Again $h$ equals zero at an elevation of $H_{S}$ relative to the Earth's surface.

The velocity of the nacelle center of mass (D) in the inertial reference frame, ${ }^{\boldsymbol{E}} \boldsymbol{v}$, is:

$$
{ }^{E} v^{D}={ }^{E} v^{o}+{ }^{E} \omega^{N} \times r^{O D}
$$

where ${ }^{E} \omega^{N}$ is the angular velocity of the nacelle in the inertial reference frame $\left({ }^{E} \omega^{N}={ }^{E} \omega^{B}+\right.$ $\left.{ }^{B} \omega^{N}\right)$. Similarly, the velocity of the teeter pin (P) in the inertial reference frame, ${ }^{E} v^{P}$, is:

$$
{ }^{E} v^{P}={ }^{E} v^{O}+{ }^{E} \omega^{N} \times r^{O P}
$$

\footnotetext{
${ }^{19}$ This is exactly the same expression for the velocity of the tower-top base plate in the inertial reference frame given by Wilson et al. (1999) and coded into FAST_AD. Since FAST_AD assumes that the towers are relatively rigid, and consequently, that deflection is small, this approximation introduces negligible errors. For relatively soft towers, however, this approximation may not fare so well.
} 
The velocities of the blade axes intersection point (Q) and the hub center of mass (C) in the inertial reference frame, ${ }^{E} v^{Q}$ and ${ }^{E} v^{C}$ respectively, are:

$$
\begin{gathered}
{ }^{E} v^{Q}={ }^{E} v^{P}+{ }^{E} \omega^{H} \times r^{P Q} \\
\text { and } \\
{ }^{E} v^{C}={ }^{E} v^{P}+{ }^{E} \omega^{H} \times r^{P C}
\end{gathered}
$$

Finally, the velocity of any point $S$ on blade 1 in the inertial reference frame, ${ }^{E} v^{S}$, is:

$$
{ }^{E} v^{S}={ }^{E} v^{Q}+{ }^{H} v^{S}+{ }^{E} \omega^{H} \times r^{Q S}
$$

where ${ }^{H} \boldsymbol{v}^{S}$ is the velocity of the point S on blade 1 relative to the rotating reference frame fixed in the hub (rotor) which is simply the time derivative of equation (3.29) performed while holding the $i$ 's constant:

$$
{ }^{\boldsymbol{H}} \boldsymbol{v}^{\boldsymbol{S}}=\dot{u}(r, t) \boldsymbol{i}_{1}+\dot{v}(r, t) \boldsymbol{i}_{2}-\dot{w}(r, t) \boldsymbol{i}_{3}
$$

Many of the previously developed equations can be combined to expand the velocity of any point $\mathrm{S}$ on blade 1 in the inertial reference frame ${ }^{20}$ :

$$
\begin{aligned}
{ }^{E} \boldsymbol{v}^{S}= & \left(\dot{q}_{7}+\dot{q}_{9}\right) \boldsymbol{a}_{1}+\left(\dot{q}_{8}+\dot{q}_{10}\right) \boldsymbol{a}_{3}+\left(\dot{\theta}_{8} \boldsymbol{a}_{1}+\dot{\theta}_{7} \boldsymbol{a}_{3}+\dot{q}_{6} \boldsymbol{d}_{2}+\dot{q}_{5} \boldsymbol{d}_{3}\right) \times \boldsymbol{r}^{\boldsymbol{O P}}+ \\
& \left(\dot{\theta}_{8} \boldsymbol{a}_{1}+\dot{\theta}_{7} \boldsymbol{a}_{3}+\dot{q}_{6} \boldsymbol{d}_{2}+\dot{q}_{5} \boldsymbol{d}_{3}+\dot{q}_{4} \boldsymbol{d}_{1}+\dot{q}_{3} \boldsymbol{g}_{2}\right) \times \boldsymbol{r}^{P Q}+ \\
& \left(\dot{\theta}_{8} \boldsymbol{a}_{1}+\dot{\theta}_{7} \boldsymbol{a}_{3}+\dot{q}_{6} \boldsymbol{d}_{2}+\dot{q}_{5} \boldsymbol{d}_{3}+\dot{q}_{4} \boldsymbol{d}_{1}+\dot{q}_{3} \boldsymbol{g}_{2}\right) \times \boldsymbol{r}^{Q S}+{ }^{H} \boldsymbol{v}^{S}
\end{aligned}
$$

When expanded as in Eq. (3.92), all the velocities of the various points on the wind turbine system in the inertial reference frame can be expressed as:

$$
{ }^{E} \boldsymbol{v}^{X}=\left(\sum_{r=1}^{15}{ }^{E} \boldsymbol{v}_{r}^{X} \dot{q}_{r}\right)+{ }^{E} \boldsymbol{v}_{t}^{X}
$$

${ }^{20}$ Wilson et al. (1999) expand the velocity of any point $\mathrm{S}$ on blade 1 in the inertial reference frame:

$$
\begin{aligned}
{ }^{E} \boldsymbol{v}^{S}= & \left(\dot{q}_{7}+\dot{q}_{9}\right) \boldsymbol{a}_{1}+\left(\dot{q}_{8}+\dot{q}_{10}\right) \boldsymbol{a}_{3}+\left(\dot{\theta}_{8} \boldsymbol{a}_{1}+\dot{\theta}_{7} \boldsymbol{a}_{3}+\dot{q}_{6} \boldsymbol{d}_{2}+\dot{q}_{5} \boldsymbol{d}_{3}\right) \times \boldsymbol{r}^{\boldsymbol{O P}}+ \\
& \left(\dot{\theta}_{8} \boldsymbol{a}_{1}+\dot{\theta}_{7} \boldsymbol{a}_{3}+\dot{q}_{6} \boldsymbol{d}_{2}+\dot{q}_{5} \boldsymbol{d}_{3}+\dot{q}_{4} \boldsymbol{d}_{1}+\dot{q}_{3} \boldsymbol{g}_{2}\right) \times \boldsymbol{r}^{\boldsymbol{P Q}}+ \\
& \left(\dot{\theta}_{8} \boldsymbol{a}_{1}+\dot{\theta}_{7} \boldsymbol{a}_{3}+\dot{q}_{6} \boldsymbol{d}_{2}+\dot{q}_{5} \boldsymbol{d}_{3}+\dot{q}_{4} \boldsymbol{d}_{1}+\dot{q}_{3} \boldsymbol{g}_{2}\right) \times \boldsymbol{r}{ }^{Q S}+{ }^{H} \boldsymbol{v}^{S}+ \\
& \left(\dot{\theta}_{8} \boldsymbol{a}_{1}+\dot{\theta}_{7} \boldsymbol{a}_{3}+\dot{q}_{6} \boldsymbol{d}_{2}+\dot{q}_{5} \boldsymbol{d}_{3}+\dot{q}_{4} \boldsymbol{d}_{1}+\dot{q}_{3} \boldsymbol{g}_{2}\right) \times\left(r \boldsymbol{i}_{3}\right)
\end{aligned}
$$

This equation is clearly in error caused by the additional line of terms (bottom row). However, according to code developers of FAST_AD, Eq. (3.92) is actually implemented in the FAST_AD design code. These code developers should consider revising this equation in the FAST_AD user's manual. 
where ${ }^{E} \boldsymbol{v}_{r}{ }_{\boldsymbol{r}}$ is the $r^{\text {th }}$ partial velocity ${ }^{21}$ associated with point X, which can be a function of time and the generalized coordinates but not of their time derivatives, and ${ }^{\boldsymbol{E}_{\boldsymbol{V}} \boldsymbol{X}}{ }_{\boldsymbol{t}}$ is the sum of all the terms not of this form. Similarly, the angular velocity of any reference frame $X$ in the inertial reference frame, ${ }^{\boldsymbol{E}} \boldsymbol{\omega}^{\boldsymbol{X}}$, can be expressed as:

$$
{ }^{E} \omega^{X}=\left(\sum_{r=1}^{15}{ }^{E} \omega_{r}^{X} \dot{q}_{r}\right)+{ }^{E} \omega_{t}^{X}
$$

where ${ }^{E} \omega^{X}{ }_{r}$ is the $r^{\text {th }}$ partial angular velocity associated with reference frame X, which can be a function of time and the generalized coordinates but not of their time derivatives, and ${ }^{E} \omega^{X}{ }_{t}$ is the sum of all the terms not of this form.

As an example, the partial velocities associated with a point $\mathrm{T}$ in the tower are:

$$
\boldsymbol{E}_{\boldsymbol{r}} \boldsymbol{v}_{\boldsymbol{T}}=\left\{\begin{array}{ll}
\phi_{I T}(h) \boldsymbol{a}_{1} & \text { for } r=7 \\
\phi_{2 T}(h) \boldsymbol{a}_{1} & \text { for } r=9 \\
\phi_{1 T}(h) \boldsymbol{a}_{3} & \text { for } r=8 \\
\phi_{2 T}(h) \boldsymbol{a}_{3} & \text { for } r=10 \\
0 & \text { otherwise }
\end{array} \quad(r=1,2, \ldots, 15)\right.
$$

It is also evident that:

$$
{ }^{E} v_{t}^{T}=0
$$

${ }^{21}$ To be technically correct, the definition of partial velocities (the ${ }^{\boldsymbol{E}} \boldsymbol{v}_{\boldsymbol{r}}{ }^{\text {s }}$ ) arise when the velocity of a point $\mathrm{X}$ in inertial frame $\mathrm{E}$ is expressed as:

$$
{ }^{\boldsymbol{E}} \boldsymbol{v}^{\boldsymbol{X}}=\left(\sum_{r=1}^{N}{ }^{\boldsymbol{E}} \boldsymbol{v}_{r}^{X} u_{r}\right)+{ }^{\boldsymbol{E}} \boldsymbol{v}_{t}^{X}
$$

where $N$ is the number of DOFs required to specify the position of the entire system under consideration and $u_{r}$ is the $r^{\text {th }}$ generalized speed. A generalized speed is essentially a composite of the velocities of the generalized coordinates:

$$
u_{r}=\left(\sum_{s=1}^{N} Y_{r s} \dot{q}_{s}\right)+Z_{r} \quad(r=1,2, \cdots, N)
$$

where the coefficients $Y_{r s}$ can be a function of time and the generalized coordinates but not of their time derivatives and the terms $Z_{r}$ are the sum of all of the terms not of this form. Thus, the labeling of partial velocities as in Eq. (3.93) is equally valid if $Y_{r s}$ and $Z_{r}$ are chosen:

$$
\begin{gathered}
Y_{r s}=\left\{\begin{array}{cc}
1 & \text { for } s=r \\
0 & \text { otherwise }
\end{array} \quad(r, s=1,2, \cdots, N)\right. \\
Z_{r}=0 \quad(r=1,2, \cdots, N)
\end{gathered}
$$


Accelerations can be found by taking time derivatives of the velocities. When differentiating Eq. (3.93) with respect to time, one arrives at the acceleration of any point $\mathrm{X}$ in the inertial frame, $\boldsymbol{E}^{X}$ :

$$
{ }^{\boldsymbol{E}} \boldsymbol{a}^{\boldsymbol{X}}=\left(\sum_{r=1}^{15}{ }^{\boldsymbol{E}} \boldsymbol{v}_{r}^{X} \ddot{q}_{r}\right)+\left[\sum_{r=1}^{15} \frac{d}{d t}\left({ }^{\boldsymbol{E}} \boldsymbol{v}_{r}^{X}\right) \dot{q}_{r}\right]+\frac{d}{d t}\left({ }^{\boldsymbol{E}} \boldsymbol{v}_{t}^{X}\right)
$$

or alternatively:

$$
{ }^{\boldsymbol{E}} \boldsymbol{a}^{\boldsymbol{X}}=\left(\sum_{r=1}^{15}{ }^{\boldsymbol{E}} \boldsymbol{v}_{r}^{X} \ddot{q}_{r}\right)+{ }^{\boldsymbol{E}} \boldsymbol{a}_{t}^{X}
$$

where,

$$
{ }^{E} \boldsymbol{a}_{t}^{X}=\left[\sum_{r=1}^{15} \frac{d}{d t}\left({ }^{E} \boldsymbol{v}_{r}^{X}\right) \dot{q}_{r}\right]+\frac{d}{d t}\left({ }^{E} \boldsymbol{v}_{t}^{X}\right)
$$

\subsection{Kinetics}

The kinematics expressions for the entire wind turbine structure found in section 3.3 can be used to form kinetics expressions. Kane's equations of motion (see section 3.5) use two sets of scalar quantities called generalized inertia forces, $F_{r}{ }^{*}$ 's, and generalized active forces, $F_{r}$ 's:

$$
\begin{gathered}
F_{r}{ }^{*}=\sum_{i=1}^{v}{ }^{E} \boldsymbol{v}_{r}^{\boldsymbol{X}_{i}} \cdot\left(-m_{X_{i}}{ }^{E} \boldsymbol{a}^{\boldsymbol{X}_{\boldsymbol{i}}}\right) \quad(r=1,2, \ldots, 15) \\
\text { and } \\
F_{r}=\sum_{i=1}^{v}{ }^{E} \boldsymbol{v}_{r}^{\boldsymbol{X}_{\boldsymbol{i}}} \cdot\left(\boldsymbol{F}^{\boldsymbol{X}_{\boldsymbol{i}}}\right) \quad(r=1,2, \ldots, 15)
\end{gathered}
$$

where $v$ is the number of particles with mass in the system, ${ }^{\boldsymbol{E}} \boldsymbol{v}^{X i}{ }_{r}$ is the $r^{\text {th }}$ partial velocity associated with particle $X_{i}, m_{X i}$ is the mass of particle $X_{i},{ }^{\boldsymbol{E}} \boldsymbol{a}^{\boldsymbol{X} \boldsymbol{i}}$ is the acceleration of particle $X_{i}$ in the inertial frame, and $\boldsymbol{F}^{X i}$ is the resultant of all applied forces acting on particle $X_{i}$. If the system is composed of a number of rigid bodies instead of particles, the generalized inertia forces can be simplified:

$$
F_{r}{ }^{*}=\sum_{i=1}^{w}{ }^{E} \boldsymbol{v}_{r}^{X_{i}} \cdot\left(-m_{X_{i}}{ }^{E} a^{X_{i}}\right)+{ }^{E} \boldsymbol{\omega}_{r}^{X_{i}} \cdot\left(-{ }^{E} \dot{\boldsymbol{H}}^{X_{i}}\right) \quad(r=1,2, \ldots, 15)
$$

where $w$ is the number of rigid bodies with mass in the system, ${ }^{\boldsymbol{E}} \boldsymbol{v}^{\boldsymbol{X} \boldsymbol{i}}{ }_{\boldsymbol{r}}$ is the $r^{\text {th }}$ partial velocity associated with the center of mass of rigid body $X_{i}, m_{X i}$ is the mass of rigid body $X_{i},{ }^{\boldsymbol{E}} \boldsymbol{a}^{\boldsymbol{X} \boldsymbol{i}}$ is the acceleration of the center of mass of rigid body $X_{i}$ in the inertial frame, ${ }^{E} \omega^{X i}{ }_{r}$ is the $r^{\text {th }}$ partial 
angular velocity associated with rigid body $X_{i}$, and ${ }^{\boldsymbol{E}} \hat{\boldsymbol{H}}^{X \boldsymbol{i}}$ is the time derivative of the angular momentum of rigid body $X_{i}$ about its center of mass, in the inertial frame. For ease in computation, ${ }^{\boldsymbol{E}} \hat{\boldsymbol{H}}^{X i}$ can be written in terms of a body-fixed coordinate system ${ }^{22}$ :

$$
{ }^{E} \dot{\boldsymbol{H}}^{X_{i}}=\left(\dot{H}^{X_{i}}\right)^{\prime}+{ }^{E} \omega^{X_{i}} \times{ }^{E} H^{X_{i}}
$$

where $\left(\hat{\boldsymbol{H}}^{X \boldsymbol{i}}\right)$ ' is the time derivative of the angular momentum of rigid body $X_{i}$ about its center of mass, relative to the body-fixed coordinate system $\left[\left(\hat{\boldsymbol{H}}^{X i}\right)\right.$ ' contains time derivatives of the angular velocity of the body but the moments and products of inertia are all constant], ${ }^{E} \omega^{X i}$ is the angular velocity associated with rigid body $X_{i}$ in the inertial frame (equal to the angular velocity of the body-fixed coordinate system in the inertial frame), and ${ }^{E} \boldsymbol{H}^{X i}$ is the angular momentum of rigid body $X_{i}$ about its center of mass, in the inertial frame.

For the wind turbine modeled in FAST_AD, the mass of the tower, nacelle, hub, and blades contribute to the total generalized inertia forces:

$$
F_{r}^{*}=\left.F_{r} *\right|_{T}+\left.F_{r} *\right|_{N}+\left.F_{r} *\right|_{H}+\left.F_{r} *\right|_{B} \quad(r=1,2, \ldots, 15)
$$

The generalized inertia forces associated with the tower, $F_{r}{ }_{T}$, result from the tower's distributed lineal density, $\mu_{T}(h)$ :

$$
\left.F_{r} *\right|_{T}=-\int_{0}^{H} \mu_{T}(h)^{E} \boldsymbol{v}_{r}^{\boldsymbol{T}} \cdot{ }^{\boldsymbol{E}} \boldsymbol{a}^{\boldsymbol{T}} d h \quad(r=1,2, \ldots, 15)
$$

where ${ }^{E} \boldsymbol{v}_{r}^{T}$ is the $r^{\text {th }}$ partial velocity associated with point $\mathrm{T}$ in the tower and ${ }^{\boldsymbol{E}} \boldsymbol{a}^{\boldsymbol{T}}$ is the acceleration of the same point in the inertial frame.

Tower deflections, yaw rates, and tilt rates contribute to the generalized inertia forces associated with the nacelle, $\left.F_{r}{ }^{*}\right|_{D}$ :

$$
\left.F_{r} *\right|_{N}={ }^{E} \boldsymbol{v}_{r}^{D} \cdot\left(-m_{N}{ }^{E} \boldsymbol{a}^{\boldsymbol{D}}\right)+{ }^{E} \boldsymbol{\omega}_{r}^{N} \cdot\left(-{ }^{\boldsymbol{E}} \dot{\boldsymbol{H}}^{\boldsymbol{D}}\right) \quad(r=1,2, \ldots, 15)
$$

where ${ }^{E} \boldsymbol{v}_{\boldsymbol{r}}{ }_{\boldsymbol{r}}$ is the $r^{\text {th }}$ partial velocity associated with the center of mass (point D) of the nacelle, $m_{N}$ is the mass of the nacelle, ${ }^{\boldsymbol{E}} \boldsymbol{a}^{\boldsymbol{D}}$ is the acceleration of the center of mass of the nacelle in the inertial frame, ${ }^{E} \omega^{N}{ }_{r}$ is the $r^{\text {th }}$ partial angular velocity associated with the nacelle, and ${ }^{\boldsymbol{E}} \hat{\boldsymbol{H}}^{\boldsymbol{D}}$ is the time derivative of angular momentum of the nacelle about its center of mass, in the inertial frame.

\footnotetext{
${ }^{22}$ FAST_AD employs dyadic notation, in which case ${ }^{E} \hat{\boldsymbol{H}}^{X \boldsymbol{i}}$ is written:$$
{ }^{E} \dot{\mathrm{H}}^{X_{i}}=I^{X_{i}}{ }^{E} \dot{\omega}^{X_{i}}+{ }^{E} \omega^{X_{i}} \times I^{X_{i}} \cdot{ }^{E} \omega^{X_{i}}
$$

where $I^{X i}$ is the inertia dyadic of rigid body $X_{i}$ about its center of mass, relative to the body-fixed coordinate system (i.e., $\boldsymbol{I}^{X i}$, which contains the moments and products of inertia, is constant), ${ }^{E} \boldsymbol{\omega}^{X i}$ is the angular acceleration associated with rigid body $X_{i}$ in the inertial frame, and ${ }^{E} \omega^{X i}$ is the angular velocity associated with rigid body $X_{i}$ in the inertial frame (equal to the angular velocity of the body-fixed coordinate system in the inertial frame).
} 
Similarly, the generalized inertia forces associated with the hub, $F_{r}{ }^{*}{ }_{H}$, are:

$$
\left.F_{r} *\right|_{H}={ }^{E} \boldsymbol{v}_{r}^{C} \cdot\left(-m_{H}{ }^{E} \boldsymbol{a}^{C}\right)+{ }^{E} \boldsymbol{\omega}_{r}^{H} \cdot\left(-{ }^{E} \dot{\boldsymbol{H}}^{C}\right) \quad(r=1,2, \ldots, 15)
$$

where ${ }^{E} \boldsymbol{v}_{r}{ }_{r}$ is the $r^{\text {th }}$ partial velocity associated with the center of mass (point C) of the hub, $m_{H}$ is the hub mass, ${ }^{E} \boldsymbol{a}^{C}$ is the acceleration of the center of mass of the hub in the inertial frame, ${ }^{E}{ }^{\boldsymbol{\omega}^{H}}{ }_{r}$ is the $r^{\text {th }}$ partial angular velocity associated with the hub, and ${ }^{E} \hat{\boldsymbol{H}}^{C}$ is the time derivative of angular momentum of the hub about its center of mass, in the inertial frame.

Finally, the distributed lineal density of the blades, $\mu_{B}(r)$, contribute to the generalized inertia forces associated with the blades, $\left.F_{r}{ }^{*}\right|_{B}$, in the same way the distributed lineal density of the tower contributed to its corresponding generalized inertia forces:

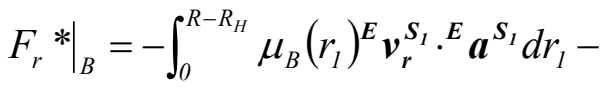

$$
\begin{aligned}
& \int_{0}^{R-R_{H}} \mu_{B}\left(r_{2}\right)^{E} \boldsymbol{v}_{r}^{S_{2}} \cdot{ }^{E} a^{S_{2}} d r_{2} \quad(r=1,2, \ldots, 15)
\end{aligned}
$$

where ${ }^{E} \boldsymbol{v}^{S i}{ }_{r}$ is the $r^{\text {th }}$ partial velocity associated with point $S$ in blade $i(i=1$ and 2$)$ and ${ }^{E} \boldsymbol{a}^{S i}$ is the acceleration of the same point in the inertial frame.

The resultant of all applied forces acting on elements of the wind turbine contributes to the total generalized active forces that govern the equations of motion. These forces include aerodynamic forces (see Chapter 2); elastic forces from the tower, blade, and drive train flexibility; elastic forces from the springs inherent in the yaw drive (yaw and tilt motion) and teetering device; gravitational forces; generator forces; and damping forces:

$$
F_{r}=\left.F_{r}\right|_{\text {Aero }}+\left.F_{r}\right|_{\text {Elastic }}+\left.F_{r}\right|_{\text {Grav }}+\left.F_{r}\right|_{\text {Generator }}+\left.F_{r}\right|_{\text {Damp }} \quad(r=1,2, \ldots, 15)
$$

An exhaustive development of all these local generalized active forces is beyond the scope of this work. Brief descriptions of a few follow.

If $\boldsymbol{F}_{\text {Aero }}{ }^{S i}$ is the resultant of all aerodynamic forces acting on point $S$ in blade $i(i=1$ and 2$)$, then the generalized active aerodynamic forces, $\left.F_{r}\right|_{A e r o}$, are:

$$
\left.F_{r}\right|_{\text {Aero }}=\int_{0}^{R-R_{H}}{ }^{E} \boldsymbol{v}_{r}^{S_{I}} \cdot \boldsymbol{F}_{\text {Aero }}^{s_{1}} d r_{1}+\int_{0}^{R-R_{H}} E^{E} \boldsymbol{v}_{r}^{S_{2}} \cdot \boldsymbol{F}_{\text {Aero }}^{s_{2}} d r_{2} \quad(r=1,2, \ldots, 15)
$$

FAST_AD assumes aerodynamic forces act only on the rotor blades. FAST_AD neglects any aerodynamic forces that are imparted on the tower or the rest of the wind turbine structure, though FAST_AD does assume that the tower forms a wake (tower shadow) that affects the aerodynamics of downwind turbines (see section 2.6).

Instead of defining the generalized active elastic restoring forces of the tower and blades with Eq. (3.101), FAST_AD uses the equivalent form: 


$$
F_{r}=\frac{\partial V}{\partial q_{r}} \quad(r=1,2, \ldots, 15)
$$

where the potential energy, $V$ :

$$
V=\frac{1}{2} \sum_{i=1}^{N} \sum_{j=1}^{N} k_{i j} q_{i}(t) q_{j}(t)
$$

where $N$ is the number of DOFs, $k_{i j}$ is the generalized stiffness, and the $q_{i}(t)$ 's are the generalized coordinates pertaining to the flexible body. There is a subtle difference between Eqs. (3.112) and (3.44). Since Eq. (3.112) contains $q_{i}(t)$ 's and Eq. (3.44) contains $c_{i}(t)$ 's, the generalized stiffness inherent in Eq. (3.112) is related to the integrals of the partial derivatives of the normal mode shapes [the $\phi_{i}(z)$ 's], whereas the generalized stiffness inherent in Eq. (3.44) is related to the integrals of the partial derivatives of the shape functions [the $\varphi_{i}(z)$ 's].

Combining the elastic restoring forces and the gravitational forces of the tower into a single set of forces, the generalized active forces attributed to the tower deflection are found by replacing the $\varphi_{i}(h)$ 's with $\phi_{i}(h)$ 's in the generalized stiffness of the tower [Eq. (3.69)], substituting the result into Eq. (3.112), and then substituting the combined result into Eq. (3.111). Since the normal mode shapes are orthogonal to each other, the cross-coupled terms will drop out of this process (the generalized stiffness terms, $k_{i j}$, involving $i \neq j$ will be zero).

A similar process is performed when computing the generalized active elastic restoring forces of the blades. However, since the centrifugal stiffening effects are accounted for in the generalized inertia force of the blades [see Eq. (3.108)], the centrifugal stiffening terms must be dropped from the generalized stiffness of the blades given in Eq. (3.77). The potential energy of the flexing beam is the only potential energy term substituted into Eq. (3.111) when finding the generalized active elastic restoring forces of the blades. Since the centrifugal force terms are included when calculating the natural mode shapes but not in the immediate discussion of the generalized blade stiffness, the cross-coupled generalized stiffness terms will not drop out.

Structural damping inherent in the tower and blades is modeled in FAST_AD using the Rayleigh damping technique. To characterize the magnitude of this damping, modal damping ratios are defined in the FAST_AD input file.

The flexibility of the drive shaft is modeled with a linear torsional spring and a linear viscous damper. The flexibility of the yaw drive, which allows everything atop the tower-top base plate to yaw and tilt, is modeled with a linear torsional spring and a linear torsional viscous damper in both the yaw and tilt directions. The teetering device is modeled with a constant coulomb damping moment (that is, constant in magnitude, but always opposing the teeter motion), a linear torsional viscous damper, and a nonlinear, cubic torsional spring. A teeter stop, which prevents excessive teeter motion, is modeled with a linear spring. 


\subsection{Kane's Equations of Motion}

FAST_AD uses Kane's method to set up equations of motion, which are then solved by numerical integration (see Chapter 4). By a direct result of Newton's laws of motion, Kane's equations of motion for a simple holonomic system can be stated as (Kane and Levinson 1985):

$$
F_{r}+F_{r}{ }^{*}=0 \quad(r=1,2, \ldots, 15)
$$

Substituting the equations presented in the previous sections of this chapter into Eq. (3.113) results in a set of 15 coupled, dynamic equations that prescribe the motion of the entire wind turbine structure as a function of time. 


\section{FAST_AD Design Code Overview and Limitations}

The FAST_AD design code is a medium-complexity code used to (1) model a wind turbine structurally given the turbine layout and aerodynamic and mechanical properties of its members and (2) simulate the wind turbine's aerodynamic and structural response by imposing complex virtual wind-inflow conditions. Outputs of these simulations include time-series data on the loads and responses of the structural members of the wind turbine. Post-processing codes can than be used to analyze these data, enabling researchers and designers to efficiently and safely design, analyze, and improve wind energy systems and lower the cost of wind-generated electricity. Wind energy researchers and designers rely heavily on computer modeling tools such as FAST_AD to accurately predict, quantify, and understand the complex physical interactions that characterize component loads and overall wind turbine performance.

FAST_AD is a streamlined code developed through a partnership between NREL and Oregon State University. The aerodynamics theories are modeled using the AeroDyn subroutine package (indicated by the AD postscript), Developed by engineering faculty and staff at the University of Utah. The FAST_AD design code can be used to model both two- and threebladed HAWTs; though as in Chapter 3, this chapter focuses solely on the two-bladed HAWT configuration. The modifications needed to extend the code to three-bladed HAWTS are beyond the scope of this work. An overview of the code is presented in section 4.1 and some of its limitations are given in section 4.2.

\subsection{FAST_AD Design Code Overview}

To simulate a wind turbine's aerodynamic and structural response to various wind-inflow conditions, FAST_AD uses BEM theory aerodynamic models and Kane's structural dynamics models as developed in Chapters 2 and 3. Before a wind turbine simulation can be performed in FAST_AD, the parameters of the models must first be defined in an input file. The main input file of FAST_AD contains 200+ parameters that must be specified. Details regarding the layout of this input file are beyond the scope of this work and are well documented in Wilson et al. (1999). An example input file is given in Appendix I for test case S0700000 of the Blind Comparison study of the UAE wind turbine. A description of the UAE wind turbine and the Blind Comparison exercise is presented in Chapter 5, and the steps needed to develop this input file are given in Chapter 6.

Many of the input file parameters pertain to the geometrical layout of the turbine and mechanical properties of its members, most of which are described thoroughly in Chapter 3 . Another group of input file parameters pertains to the initial conditions of the system (the configuration of the wind turbine at time, $t=0$ ). All 15 of the model's DOFs can be switched on or off to systematically reduce or increase the complexity of the model. The switch for the DOF variable $q_{4}$ is a bit broader, enabling the analyst to toggle between one of several generator models: constant speed, induction generator, start up, shut down, variable speed, and idling (no generator torque). Several pitch control methodologies - fixed pitch, power control, and speed controlcan also be modeled. 
Aerodynamic properties of the rotor blades form the basis of another significant fraction of input file parameters. In FAST_AD, each blade element can be modeled with its own unique airfoil table, which describes the dimensionless coefficient airfoil data $\left(C_{L}\right.$ and $\left.C_{D}\right)$ as a function of angle of attack $(\alpha)$. Since wind turbine airfoils operate in both the attached and separated flow regimes, especially under heavy winds and yawed conditions, these airfoil tables must encompass the entire $360^{\circ}$ range of potential angles of attack. The input file also enables the analyst to choose whether to include axial induction, wake rotation, tower shadow, dynamic stall, and dynamic inflow effects when simulating. The wind-inflows can be modeled as uniform, unvarying wind shear, or fully unsteady, inhomogeneous, and turbulent. Theories and descriptions of each of these simple and advanced models are presented in Chapter 2.

During a simulation, FAST_AD numerically iterates ${ }^{23}$ to find the actual operating condition of each blade element at each time segment (to find the intersection point of the BEM $C_{L}$ versus $\alpha$ curve and the $C_{L}$ versus $\alpha$ curve characterizing the aerodynamic properties of the element's airfoil; or equivalently, to find the axial and tangential induction factors). The iterative method is employed with forms of Eqs. (2.71), (2.72), and (2.53) where the dimensionless drag coefficients, the $C_{D}$ 's, are assumed negligible and are set equal to zero. Throughout this process, the velocities of the incoming flow stream relative to each blade element are adjusted from the form given in Eq. (2.52) to account for motions associated with deflections of the tower, nacelle, and blades.

Since the only other externally applied load is gravity, once the aerodynamic forces are determined, Kane's equations of motion [see Eq. (3.113)] can be used to solve for the ensuing velocities of every constituent of the wind turbine modeled. To do this, Eq. (3.113) is written as:

$$
\sum_{s=1}^{15} C_{r s} \ddot{q}_{s}+f_{r}\left(\dot{q}_{r}, q_{r}\right)=0 \quad(r=1,2, \ldots, 15)
$$

and is then expanded into matrix form:

$$
\left[\begin{array}{cccccc}
C_{11} & C_{12} & \ldots & \ldots & \ldots & C_{115} \\
C_{21} & C_{22} & & \vdots & & C_{215} \\
\vdots & & \ddots & \vdots & & \vdots \\
\vdots & \ldots & \ldots & C_{r s} & \ldots & \vdots \\
\vdots & & & \vdots & \ddots & \vdots \\
C_{151} & C_{152} & \ldots & \ldots & \ldots & C_{1515}
\end{array}\right]\left\{\begin{array}{c}
\ddot{q}_{1} \\
\ddot{q}_{2} \\
\vdots \\
\ddot{q}_{s} \\
\vdots \\
\ddot{q}_{15}
\end{array}\right\}=\left\{\begin{array}{c}
-f_{1}\left(\dot{q}_{1}, q_{1}\right) \\
-f_{2}\left(\dot{q}_{2}, q_{2}\right) \\
\vdots \\
-f_{r}\left(\dot{q}_{r}, q_{r}\right) \\
\vdots \\
-f_{15}\left(\dot{q}_{15}, q_{15}\right)
\end{array}\right\}
$$

where the $C_{r s}$ 's are known coefficients of the accelerations of each DOF variable and the $f_{r}$ 's contain lower order terms. At each time step, the first step of the numerical solution to Eq. (4.2) is to use a fourth-order Adams-Beshforth predictor method to determine the lower order terms that make up the right-hand side of these equations. A Gauss elimination technique is then

\footnotetext{
${ }^{23}$ However, no iteration is necessary when employing the dynamic inflow model.
} 
performed to solve for the accelerations of the DOF variables. Next, these accelerations are used to improve the estimate made by the predictor. After several iterations, a fourth-order AdamsMounton corrector is used to make a final estimate and a final determination of the accelerations, giving the final solution for this time step. Since this predictor-corrector method is not selfstarting, a fourth-order Runge-Kutta method is used to determine the solution for the first four time steps. The solution algorithm is very efficient, so the code is indeed fast. The time step or time increment, $d t$, and time duration of the simulation, $t_{M a x}$, are additional parameters designated in the input file.

Once the applied loads and motion of the entire system are characterized for a given time step, loads (forces and moments) at various critical points on the wind turbine are found by performing simple composites (summations) of these loads. A list of the selection of output parameters available to the analyst is given in Wilson et al. (1999). Derivations of the equations used to calculate all these parameters are beyond the scope of this work. A list of desired output parameters, referred to as channels, is one of the final sets of parameters defined in the FAST_AD input file.

\subsection{FAST_AD Design Code Limitations}

The FAST_AD design code is a noteworthy tool for researchers and designers of wind energy systems. It is significantly more developed than codes such as YawDyn (Hansen and Laino 1998) and WT_Perf (Buhl 2000), enabling analysts to study the complex interactions between structural members of a wind turbine during normal and extreme operating conditions. WT_Perf is essentially a wind turbine performance prediction code only. YawDyn is more advanced than WT_Perf, permitting an analyst to model several structural degrees of freedom, but exhibits few of the capabilities available in FAST_AD. FAST_AD is also significantly less sophisticated, easier to use, and faster to operate than $\mathrm{ADAMS}^{\mathbb{R}^{-}}$, which is an advanced, commercial, multibody-dynamics code developed by Mechanical Dynamics, Inc. capable of modeling virtually any type of mechanical device.

Regardless of its attributes, omitting a discussion of FAST_AD's limitations would be careless when performing simulations in FAST_AD and when discussing the significance of the results (see Chapters 6 and 7). As presented in the wind turbine aerodynamics discussion and in Chapter 2, one limitation is that FAST_AD, or more specifically AeroDyn, regards the drag coefficient as negligible when iterating to find an aerodynamic operating point. For wind turbines consisting of aerodynamically effective blades operating in the attached flow regime, this notion is justified since drag is very small in this operating state. However, for conditions around stall and in the post-stall regime, essentially all airfoils begin to exhibit significant drag. At this point, one should be reluctant to accept all simulated results as accurate, since fundamental assumptions regarding the models from which those results were obtained were violated in the process.

Another limitation is that blade pitching moments (or equivalently feather moments) are neglected; that is, FAST_AD assumes that none of the effective aerodynamic forces acting on the blades cause the blades to twist. This prohibits FAST_AD from modeling important dynamical conditions such as flutter. Flutter is a destructive concatenation of torsional and flapping oscillations that has been known to catastrophically twist improperly designed blades 
off the pitching mechanism. For wind turbines with blades that have inherently high torsional stiffness or coincident inertial and aerodynamic pitching axes, flutter is generally negligible. With the lack of either condition, however, FAST_AD has limited ability to model this important dynamical event. Moreover, neglecting pitching moment calculations causes the predicted stress levels at the blade root to be underpredicted regardless of blade design.

FAST_AD also neglects the computation of any radial aerodynamic loads, which may contribute to blade root stresses on real wind turbines. If the radial loads or blade deflections are substantial, the absence of radial load considerations may also bring about underpredicted root bending moments.

One of FAST_AD's minor limitations is that it assumes the natural mode shapes of the tower in the longitudinal and lateral directions are identical. This limitation is deemed minor since most wind turbines use axisymmetric towers.

With similar merit, the absence of a coning angle in the generalized mass and generalized stiffness equations is another minor limitation. This should be a concern only if the blade coning angles or teeter motion are significant, which is not the case for most utility-scale wind turbines.

Another limitation is that FAST_AD regards most deflections to be small. For example, the assumption that tower deflections are small is employed in the development of Eqs. (3.4), (3.5), (3.61), and (3.63). For relatively stiff towers, this is entirely valid. For relatively soft towers, however, this assumption begets errors that may be substantial. Similarly, the assumption that rotor blade deflections are small is employed in the development of the blade deflection equations [see Eqs. (3.10) and (3.11)]. An equivalent limitation is inherent in the blade coning angles, since the determination of the blade mode shapes and centrifugal stiffening effects are heavily dependent on the assumption that the blade coning angles are small (see footnote 15). Most utility-scale wind turbines are relatively rigid; thus, these limitations are reasonably minor. As turbines become larger and inherently more flexible, however, these limitations become increasingly important and the models will have to be expanded to incorporate the effects of increasing flexibility.

Errors in the structural models employed by FAST_AD impose other limitations. These errors are covered in detail in Chapter 3 (see footnotes 9, 10, 12, and 14) and are briefly discussed here. The most significant error is the incorrect sign convention of the distributed structural pretwist angle, which defines the blades as having the mirror image of the stiffness distribution physically relevant to the modeled wind turbine (see footnote 9). For blades with substantial stiffness orientation variability, the consequences of this error are difficult to characterize.

With similar merit, the absence of the blade collective pitch angle in the equations defining the stiffness orientation of the blades gives rise to important dynamical consequences that are difficult to characterize (see footnote 12). Fixed pitch (stall-controlled) turbines and pitchcontrolled turbines that maintain small pitching angles will most likely have little sensitivity to this error. However, in cases where the pitching angles are large or change rapidly to adjust for wind gusts, etc., the dynamical outcomes are expected to be more significant. 
A less substantial error is the absence of the TWRHTOFFSET parameter in the kinematics and kinetics terms related to the equations of motion of the wind turbine system (see footnote 10). This error is less substantial since the tilting motion of typical nacelles is small; consequently, the dynamic effects coupled to this parameter when tilting (increased blade motions relative to the tower-top base plate) are also small.

The error in the equation for the generalized stiffness of the tower (see footnote 14) employed in FAST_AD and the Modes preprocessor code is also less substantial. The assumption that the lineal density of the tower is constant with elevation is unsubstantial, since the resulting mode shapes have a negligible effect on the dynamics of the rest of the wind turbine system and only a minor effect on the longitudinal and lateral moments in the tower base.

FAST_AD code developers should consider revising the code to correct for all these errors, since errors limit the validity of the solutions in many situations! An exhaustive study should also be performed on the other models and equations employed by FAST_AD and all its preprocessor and postprocessor codes (both structural and aerodynamic) not described in this report. Time constraints prevented us from studying and developing every model and equation employed by FAST_AD and all its preprocessor and postprocessor codes, though there may well be other errors. For example, both the Modes and FAST_AD programs output natural frequencies of the blades and tower, which are estimated from the distributed structural parameters input into the model. After running a few simulations, we noticed that the output frequencies from FAST_AD do not match those output by Modes when the same structural parameters are input. We concluded that there must be an error in the computation of these natural frequencies. A case for other errors, especially in determining a few output channels, is given in Chapter 7.

These errors are separate from any bugs in the coding. We did not perform an exhaustive overview of the coding.

Above all, the simulated results obtained from FAST_AD are only as good as the models from which they were derived. This has more to do with our limited understanding of the physics involved in wind turbine dynamics than our limited attempt to model all physical realizable scenarios. As discussed in Chapters 6 and 7, our limited understanding of the physics of threedimensional flow in a rotating environment overwhelms most of the limitations in FAST_AD. 


\section{UAE and Blind Comparison Overview}

In recent years, the wind turbine research community has made significant advances in simulating wind turbine operation and predicting performance and component loads. Design codes such as FAST_AD have been successfully used to improve wind energy systems and lower the cost of wind-generated electricity on a noteworthy scale. Though detailed, the models employed in these codes, such as those developed in the previous chapters, are surprisingly deficient. As discussed in Chapter 2, these deficiencies include the inability to accurately model the effects of inhomogeneous, unsteady, and turbulent inflows and operating states such as severe yaw error, dynamic stall, and blade/tower shadow interaction. Perhaps more disconcerting is that the models also tend to miss the mark when predicting loads and responses, even under relatively homogeneous and steady wind-inflow conditions. This is unfortunate, since the entire spectrum of physically realizable operational states must be considered when estimating extreme loads needed in the successful design of long-lasting turbines. Moreover, if a design is to be truly optimized, load predictions from all operating states must be accurate. Currently, wind turbine designers rely heavily on time-tested safety factors to account for these deficiencies. Such a design methodology greatly limits the cost effectiveness of new wind turbine designs.

To address these issues, NREL planned and conducted the comprehensive test program known as the UAE. Its objectives are daunting: "to provide accurate and reliable experimental measurements, having highly spatial and temporal resolution, for a realistic rotating blade geometry, under closely matched conditions of dynamic similarity, and in the presence of strictly controlled inflow conditions" (Simms et al. 2001). These objectives were met by testing a heavily instrumented, 10-m diameter, $20-\mathrm{kW}$ wind turbine, known as the UAE research wind turbine, in the world's largest wind tunnel located at the NASA-Ames Research Center. These tests were completed in May 2000, following three weeks of exhaustive testing. Information obtained from these tests includes data regarding the full-scale, three-dimensional steady and unsteady behavior of HAWT aerodynamics and structural dynamics including inflow conditions, wake form, airfoil aerodynamic pressure distributions, and machine responses and loads; information that is invaluable for improving and validating enhanced engineering design codes such as FAST_AD for HAWTs.

To test the validity and range of applicability of models used in design codes, NREL solicited wind turbine modeling experts from around the world to predict loads and performance of the UAE wind turbine at selected operating conditions. These predictions were done "blind" in that the only information given to the participants regarded basic machine parameters of the UAE wind turbine and essential wind-inflow data, not measured wind tunnel experimental data. During a later comparison phase, the predictions from all the Blind Comparison modelers were presented and compared with the experimentally measured data.

A summary of the UAE wind turbine configuration and technical specifications is presented in section 5.1. An overview of the wind tunnel tests and the quality and reliability of the resulting data are given in section 5.2. A description of the Blind Comparison exercise is presented in section 5.3. 


\subsection{UAE Wind Turbine Specifications}

Since the UAE wind turbine was designed specifically for research and testing, extensive documentation on the machine's configuration and its technical specifications is readily available (see Simms et al. 1999; Hand 2001; and Hand et al. 2001). An exhaustive accounting of the machine's parameters will not be presented here. This section will summarize some of the wind turbine's key characteristics that are needed to develop the input files, specifically for FAST_AD and its configuration during the Blind Comparison operating conditions. The approximations and assumptions made and the steps taken to transform these data into specific input file entries are given in Chapter 6. A discussion of the tools and techniques used to obtain the data is beyond the scope of this work.

The basic machine characteristics include:

- Number of blades: 2

- Rotor configuration: upwind or downwind (to transition from a downwind to upwind configuration, the nacelle is rotated $180^{\circ}$ and both blades are detached from the hub, rotated $180^{\circ}$, and reattached to keep the suction sides of the blades downwind)

- Power regulation: stall controlled (fixed pitch)

- Rotor type: teetered or rigid (to transition from a teetered rotor to a rigid hub configuration, the teeter dampers are removed and replaced with rigid links); the test cases considered for the Blind Comparison exercise employed only the rigid rotor type

- Rotor rotational direction: counterclockwise when looking downwind (for both rotor configurations)

- Rated turbine power: $19.8 \mathrm{~kW}$

- Cut-in wind speed $6 \mathrm{~m} / \mathrm{s}$

- Hub height above ground level: $12.192 \mathrm{~m}$

- Blade tip radius measured from hub centerline: $5.029 \mathrm{~m}$

- Distance to blade root mounted strain gages measured from hub centerline: $0.432 \mathrm{~m}$

- Distance from yaw axis to blade axis (yaw moment arm): $1.401 \mathrm{~m}$

- Distance from yaw axis to teeter pin (load path): $1.469 \mathrm{~m}$

- Synchronous rotational speed of low-speed shaft: $71.63 \mathrm{rpm}$

- Nacelle tilt angle: $0^{\circ}$

- Blade precone angles: locked at one of three positions: $0^{\circ}, 3.4^{\circ}$, or $18^{\circ}$

- Blade collective pitch angles: locked at one of five positions: $0^{\circ}, 2^{\circ}, 3^{\circ}, 4^{\circ}$, or $6^{\circ}$ measured relative to the blade tip; the test cases considered for the Blind Comparison exercise all employed the $3^{\circ}$ blade collective tip pitch position

Each rotor blade of the UAE wind turbine is tapered and twisted (see Table 5.1). All local twist angles are relative to the point of zero twist at the $3.772 \mathrm{~m}$ station $(75 \% \mathrm{span})$ and convention is positive toward feather (same as pitch convention). Between the $0.508 \mathrm{~m}$ and $0.883 \mathrm{~m}$ stations, the blade root is cylindrical. This transitions to an airfoil shape between the $0.883 \mathrm{~m}$ and 1.257 m stations. 
Table 5.1: Blade Chord and Twist Distributions

\begin{tabular}{|l|l|r|r|r|}
\hline $\begin{array}{l}\text { Distance from } \\
\text { Rotor Center } \\
m\end{array}$ & $\begin{array}{l}\text { Spanwise } \\
\text { Fraction } \\
(/ 5.029 \mathrm{~m})\end{array}$ & $\begin{array}{r}\text { Local Chord } \\
\text { Length }(c) \\
m\end{array}$ & $\begin{array}{r}\text { Local } \\
\text { Twist }\left(\theta_{T}\right) \\
\text { deg }\end{array}$ & $\begin{array}{r}\text { Local } \\
\text { Thickness } \\
m\end{array}$ \\
\hline 0.000 & 0.000 & hub & hub & hub \\
\hline 0.508 & 0.101 & 0.218 & 0.000 & 0.218 \\
\hline 0.660 & 0.131 & 0.218 & 0.000 & 0.218 \\
\hline 0.883 & 0.176 & 0.183 & 0.000 & 0.183 \\
\hline 1.008 & 0.200 & 0.349 & 6.700 & 0.163 \\
\hline 1.067 & 0.212 & 0.441 & 9.900 & 0.154 \\
\hline 1.133 & 0.225 & 0.544 & 13.400 & 0.154 \\
\hline 1.257 & 0.250 & 0.737 & 20.040 & 0.154 \\
\hline 1.343 & 0.267 & 0.728 & 18.074 & 0.153 \\
\hline 1.510 & 0.300 & 0.711 & 14.292 & 0.149 \\
\hline 1.648 & 0.328 & 0.697 & 11.909 & 0.146 \\
\hline 1.952 & 0.388 & 0.666 & 7.979 & 0.140 \\
\hline 2.257 & 0.449 & 0.636 & 5.308 & 0.133 \\
\hline 2.343 & 0.466 & 0.627 & 4.715 & 0.131 \\
\hline 2.562 & 0.509 & 0.605 & 3.425 & 0.127 \\
\hline 2.867 & 0.570 & 0.574 & 2.083 & 0.120 \\
\hline 3.172 & 0.631 & 0.543 & 1.150 & 0.114 \\
\hline 3.185 & 0.633 & 0.542 & 1.115 & 0.114 \\
\hline 3.476 & 0.691 & 0.512 & 0.494 & 0.107 \\
\hline 3.781 & 0.752 & 0.482 & -0.015 & 0.101 \\
\hline 4.023 & 0.800 & 0.457 & -0.381 & 0.096 \\
\hline 4.086 & 0.812 & 0.451 & -0.475 & 0.094 \\
\hline 4.391 & 0.873 & 0.420 & -0.920 & 0.088 \\
\hline 4.696 & 0.934 & 0.389 & -1.352 & 0.081 \\
\hline 4.780 & 0.950 & 0.381 & -1.469 & 0.080 \\
\hline 5.000 & 0.994 & 0.358 & -1.775 & 0.075 \\
\hline 5.029 & 1.000 & 0.355 & -1.815 & 0.074 \\
\hline & & & & \\
\hline & & & & \\
\hline
\end{tabular}

Except at the root region, the entire blade exhibits an NREL S809 airfoil shape (blade profile). Airfoils of this shape have been extensively tested in wind tunnels to obtain two-dimensional aerodynamic coefficients. The dimensionless coefficient airfoil data resulting from tests taken at Colorado State University (CSU), Ohio State University (OSU), and the Delft University of Technology (DUT) are given in Tables 5.2-5.7 and are illustrated in Figs. 5.1 and 5.2. 
Table 5.2: NREL S809 Airfoil Data from CSU; $R e=300,000$

\begin{tabular}{|l|r|r|}
\hline $\begin{array}{l}\text { Angle of } \\
\text { Attack }(\alpha) \\
d e g\end{array}$ & $\begin{array}{r}\text { Dim.less } \\
\text { Lift Coeff. } \\
\left(C_{L}\right)\end{array}$ & $\begin{array}{r}\text { Dim.less } \\
\text { Drag Coeff. } \\
\left(C_{D}\right)\end{array}$ \\
\hline 0.00 & 0.105 & 0.0117 \\
\hline 1.99 & 0.307 & 0.0116 \\
\hline 4.08 & 0.545 & 0.0139 \\
\hline 6.11 & 0.748 & 0.0135 \\
\hline 8.14 & 0.880 & 0.0198 \\
\hline 10.20 & 0.878 & 0.0360 \\
\hline 11.20 & 0.870 & 0.0446 \\
\hline 12.20 & 0.854 & 0.0496 \\
\hline 13.10 & 0.877 & 0.0619 \\
\hline 14.10 & 0.894 & 0.0731 \\
\hline 15.20 & 0.891 & 0.0865 \\
\hline 16.30 & 0.745 & 0.2200 \\
\hline 17.20 & 0.591 & 0.2480 \\
\hline 18.10 & 0.592 & 0.2650 \\
\hline 19.20 & 0.580 & 0.2790 \\
\hline 20.20 & 0.604 & 0.2980 \\
\hline 22.10 & 0.588 & 0.3230 \\
\hline 26.20 & 0.669 & 0.4120 \\
\hline 30.20 & 0.946 & 0.6330 \\
\hline 35.20 & 1.020 & 0.7990 \\
\hline 40.30 & 1.080 & 0.9830 \\
\hline 45.20 & 1.230 & 1.3100 \\
\hline 45.10 & 1.130 & 1.2100 \\
\hline 50.00 & 1.210 & 1.5100 \\
\hline 60.00 & 1.050 & 1.8600 \\
\hline 69.90 & 0.805 & 2.2700 \\
\hline 80.00 & 0.456 & 2.1600 \\
\hline 90.00 & 0.128 & 2.2400 \\
\hline & & \\
\hline
\end{tabular}


Table 5.3: NREL S809 Airfoil Data from CSU; $R e=500,000$

\begin{tabular}{|l|r|r|}
\hline $\begin{array}{l}\text { Angle of } \\
\text { Attack }(\alpha) \\
\text { deg }\end{array}$ & $\begin{array}{r}\text { Dim.less } \\
\text { Lift Coeff. } \\
\left(C_{L}\right)\end{array}$ & $\begin{array}{r}\text { Dim.less } \\
\text { Drag Coeff. } \\
\left(C_{D}\right)\end{array}$ \\
\hline-2.230 & -0.060 & 0.006 \\
\hline-0.161 & 0.156 & 0.004 \\
\hline 1.840 & 0.369 & 0.006 \\
\hline 3.880 & 0.571 & 0.008 \\
\hline 5.890 & 0.755 & 0.009 \\
\hline 7.890 & 0.860 & 0.017 \\
\hline 8.950 & 0.887 & 0.024 \\
\hline 9.910 & 0.869 & 0.035 \\
\hline 10.900 & 0.868 & 0.039 \\
\hline 12.000 & 0.894 & 0.048 \\
\hline 12.900 & 0.938 & 0.061 \\
\hline 14.000 & 0.929 & 0.074 \\
\hline 14.900 & 0.908 & 0.080 \\
\hline 16.000 & 0.912 & 0.106 \\
\hline 17.000 & 0.655 & 0.271 \\
\hline 18.000 & 0.588 & 0.265 \\
\hline 19.000 & 0.587 & 0.281 \\
\hline 20.000 & 0.597 & 0.299 \\
\hline 22.000 & 0.603 & 0.326 \\
\hline 24.000 & 0.647 & 0.375 \\
\hline 26.000 & 0.683 & 0.419 \\
\hline 28.100 & 0.745 & 0.482 \\
\hline 30.000 & 0.824 & 0.560 \\
\hline 35.000 & 1.050 & 0.817 \\
\hline 40.000 & 1.140 & 1.030 \\
\hline 45.000 & 1.200 & 1.260 \\
\hline 50.000 & 1.120 & 1.380 \\
\hline 55.000 & 1.170 & 1.700 \\
\hline 60.000 & 1.080 & 1.870 \\
\hline 65.000 & 0.940 & 1.980 \\
\hline 70.000 & 0.857 & 2.190 \\
\hline 74.900 & 0.666 & 2.170 \\
\hline 79.900 & 0.472 & 2.210 \\
\hline 84.800 & 0.356 & 2.320 \\
\hline 89.900 & 0.142 & 2.090 \\
\hline & & \\
\hline
\end{tabular}


Table 5.4: NREL S809 Airfoil Data from CSU; $\operatorname{Re}=650,000$

\begin{tabular}{|l|r|r|}
\hline $\begin{array}{l}\text { Angle of } \\
\text { Attack }(\alpha) \\
\text { deg }\end{array}$ & $\begin{array}{r}\text { Dim.less } \\
\text { Lift Coeff. } \\
\left(C_{L}\right)\end{array}$ & $\begin{array}{r}\text { Dim.less } \\
\text { Drag Coeff. } \\
\left(C_{D}\right)\end{array}$ \\
\hline-0.25 & 0.151 & 0.002 \\
\hline 1.75 & 0.354 & 0.001 \\
\hline 3.81 & 0.561 & 0.002 \\
\hline 5.92 & 0.765 & 0.006 \\
\hline 7.94 & 0.860 & 0.015 \\
\hline 9.98 & 0.848 & 0.031 \\
\hline 11.00 & 0.892 & 0.043 \\
\hline 12.00 & 0.888 & 0.049 \\
\hline 13.00 & 0.927 & 0.043 \\
\hline 14.00 & 0.910 & 0.075 \\
\hline 15.00 & 0.910 & 0.075 \\
\hline 16.00 & 0.928 & 0.107 \\
\hline 17.00 & 0.686 & 0.278 \\
\hline 18.00 & 0.639 & 0.276 \\
\hline 19.00 & 0.576 & 0.273 \\
\hline 20.00 & 0.552 & 0.275 \\
\hline 22.00 & 0.596 & 0.323 \\
\hline 23.90 & 0.649 & 0.370 \\
\hline 26.00 & 0.680 & 0.417 \\
\hline 30.00 & 0.851 & 0.576 \\
\hline 35.00 & 1.010 & 0.789 \\
\hline 40.00 & 1.120 & 1.030 \\
\hline 45.00 & 1.120 & 1.190 \\
\hline 50.00 & 1.100 & 1.360 \\
\hline 55.30 & 1.080 & 1.580 \\
\hline 60.20 & 0.931 & 1.620 \\
\hline 65.20 & 0.968 & 2.000 \\
\hline 70.20 & 0.776 & 2.040 \\
\hline 75.20 & 0.630 & 2.130 \\
\hline 80.20 & 0.485 & 2.320 \\
\hline 85.10 & 0.289 & 2.140 \\
\hline 90.20 & 0.109 & 2.270 \\
\hline & & \\
\hline & &
\end{tabular}


Table 5.5: NREL S809 Airfoil Data from OSU; $\operatorname{Re}=750,000$

\begin{tabular}{|l|r|r|r|}
\hline Angle of & Dim.less & Dim.less & $\begin{array}{r}\text { Reynolds } \\
\text { Attack }(\alpha)\end{array}$ \\
deg & Lift Coeff. & Drag Coeff. \\
$\left(C_{L}\right)$ & $\left(C_{D}\right)$ & $\begin{array}{r}\text { Number }(R e) \\
\times 10^{6}\end{array}$ \\
\hline-20.1 & -0.56 & 0.3027 & 0.76 \\
\hline-18.1 & -0.67 & 0.3069 & 0.75 \\
\hline-16.1 & -0.79 & 0.1928 & 0.75 \\
\hline-14.2 & -0.84 & 0.0898 & 0.75 \\
\hline-12.2 & -0.70 & 0.0553 & 0.75 \\
\hline-10.1 & -0.63 & 0.0390 & 0.73 \\
\hline-8.2 & -0.56 & 0.0233 & 0.74 \\
\hline-6.1 & -0.64 & 0.0112 & 0.74 \\
\hline-4.1 & -0.42 & -0.0004 & 0.76 \\
\hline-2.1 & -0.21 & -0.0003 & 0.75 \\
\hline 0.1 & 0.05 & 0.0029 & 0.75 \\
\hline 2.0 & 0.30 & 0.0056 & 0.74 \\
\hline 4.1 & 0.54 & 0.0067 & 0.75 \\
\hline 6.2 & 0.79 & 0.0085 & 0.74 \\
\hline 8.1 & 0.90 & 0.0127 & 0.75 \\
\hline 10.2 & 0.93 & 0.0274 & 0.75 \\
\hline 11.3 & 0.92 & 0.0303 & 0.74 \\
\hline 12.1 & 0.95 & 0.0369 & 0.74 \\
\hline 13.2 & 0.99 & 0.0509 & 0.74 \\
\hline 14.2 & 1.01 & 0.0648 & 0.74 \\
\hline 15.3 & 1.02 & 0.0776 & 0.74 \\
\hline 16.3 & 1.00 & 0.0917 & 0.74 \\
\hline 17.1 & 0.94 & 0.0994 & 0.73 \\
\hline 18.1 & 0.85 & 0.2306 & 0.77 \\
\hline 19.1 & 0.70 & 0.3142 & 0.76 \\
\hline 20.1 & 0.66 & 0.3186 & 0.76 \\
\hline 22.0 & 0.70 & 0.3694 & 0.76 \\
\hline 24.1 & 0.79 & 0.4457 & 0.77 \\
\hline 26.2 & 0.88 & 0.5260 & 0.76 \\
\hline & & & \\
\hline
\end{tabular}


Table 5.6: NREL S809 Airfoil Data from OSU; $R e=1,000,000$

\begin{tabular}{|c|c|c|c|}
\hline $\begin{array}{l}\text { Angle of } \\
\text { Attack }(\alpha) \\
\text { deg }\end{array}$ & $\begin{array}{r}\text { Dim.less } \\
\text { Lift Coeff. } \\
\left(C_{L}\right)\end{array}$ & $\begin{array}{r}\text { Dim.less } \\
\text { Drag Coeff. } \\
\left(C_{D}\right)\end{array}$ & $\begin{array}{r}\text { Reynolds } \\
\text { Number }(R e) \\
\times 10^{6}\end{array}$ \\
\hline-20.1 & -0.55 & 0.2983 & 1.01 \\
\hline-18.2 & -0.65 & 0.2955 & 1.02 \\
\hline-16.2 & -0.80 & 0.1826 & 1.01 \\
\hline-14.1 & -0.79 & 0.0793 & 0.99 \\
\hline-12.1 & -0.70 & 0.0547 & 1.01 \\
\hline-10.2 & -0.63 & 0.0401 & 1.00 \\
\hline-8.2 & -0.58 & 0.0266 & 1.00 \\
\hline-6.2 & -0.61 & 0.0183 & 1.00 \\
\hline \begin{tabular}{|l|}
-4.1 \\
\end{tabular} & -0.40 & 0.0004 & 0.99 \\
\hline-2.1 & -0.16 & 0.0009 & 1.00 \\
\hline 0.0 & 0.07 & 0.0022 & 1.01 \\
\hline 2.1 & 0.30 & 0.0037 & 1.00 \\
\hline 4.1 & 0.55 & 0.0050 & 1.00 \\
\hline 6.1 & 0.79 & 0.0063 & 1.00 \\
\hline 8.2 & 0.90 & 0.0096 & 1.00 \\
\hline 10.1 & 0.94 & 0.0231 & 1.00 \\
\hline 11.2 & 0.93 & 0.0236 & 1.00 \\
\hline 12.2 & 0.97 & 0.0368 & 1.00 \\
\hline 13.3 & 1.00 & 0.0551 & 0.99 \\
\hline 14.2 & 1.02 & 0.0618 & 0.99 \\
\hline 15.2 & 1.03 & 0.0705 & 0.99 \\
\hline 16.2 & 1.01 & 0.0880 & 1.00 \\
\hline 17.2 & 0.95 & 0.1043 & 0.99 \\
\hline 18.1 & 0.90 & 0.1325 & 1.00 \\
\hline 19.2 & 0.78 & 0.3474 & 1.02 \\
\hline 20.0 & 0.67 & 0.3211 & 1.02 \\
\hline 22.1 & 0.70 & 0.3699 & 1.02 \\
\hline 24.0 & 0.77 & 0.4348 & 1.03 \\
\hline 26.1 & 0.91 & 0.5356 & 1.04 \\
\hline
\end{tabular}


Table 5.7: NREL S809 Airfoil Data from DUT; $R e=1,000,000$

\begin{tabular}{|l|r|r|}
\hline $\begin{array}{l}\text { Angle of } \\
\text { Attack }(\alpha) \\
\text { deg }\end{array}$ & $\begin{array}{r}\text { Dim.less } \\
\text { Lift Coeff. } \\
\left(C_{L}\right)\end{array}$ & $\begin{array}{r}\text { Dim.less } \\
\text { Drag Coeff. } \\
\left(C_{D}\right)\end{array}$ \\
\hline-1.04 & 0.019 & 0.0095 \\
\hline-0.01 & 0.139 & 0.0094 \\
\hline 1.02 & 0.258 & 0.0096 \\
\hline 2.05 & 0.378 & 0.0099 \\
\hline 3.07 & 0.497 & 0.0100 \\
\hline 4.10 & 0.617 & 0.0100 \\
\hline 5.13 & 0.736 & 0.0097 \\
\hline 6.16 & 0.851 & 0.0095 \\
\hline 7.18 & 0.913 & 0.0127 \\
\hline 8.20 & 0.952 & 0.0169 \\
\hline 9.21 & 0.973 & 0.0247 \\
\hline 10.20 & 0.952 & 0.0375 \\
\hline 11.21 & 0.947 & 0.0725 \\
\hline 12.23 & 1.007 & 0.0636 \\
\hline 13.22 & 1.031 & 0.0703 \\
\hline 14.23 & 1.055 & 0.0828 \\
\hline 15.23 & 1.062 & 0.1081 \\
\hline 16.22 & 1.043 & 0.1425 \\
\hline 17.21 & 0.969 & 0.1853 \\
\hline 18.19 & 0.938 & 0.1853 \\
\hline 19.18 & 0.929 & 0.1853 \\
\hline 20.16 & 0.923 & 0.1853 \\
\hline & & \\
\hline
\end{tabular}

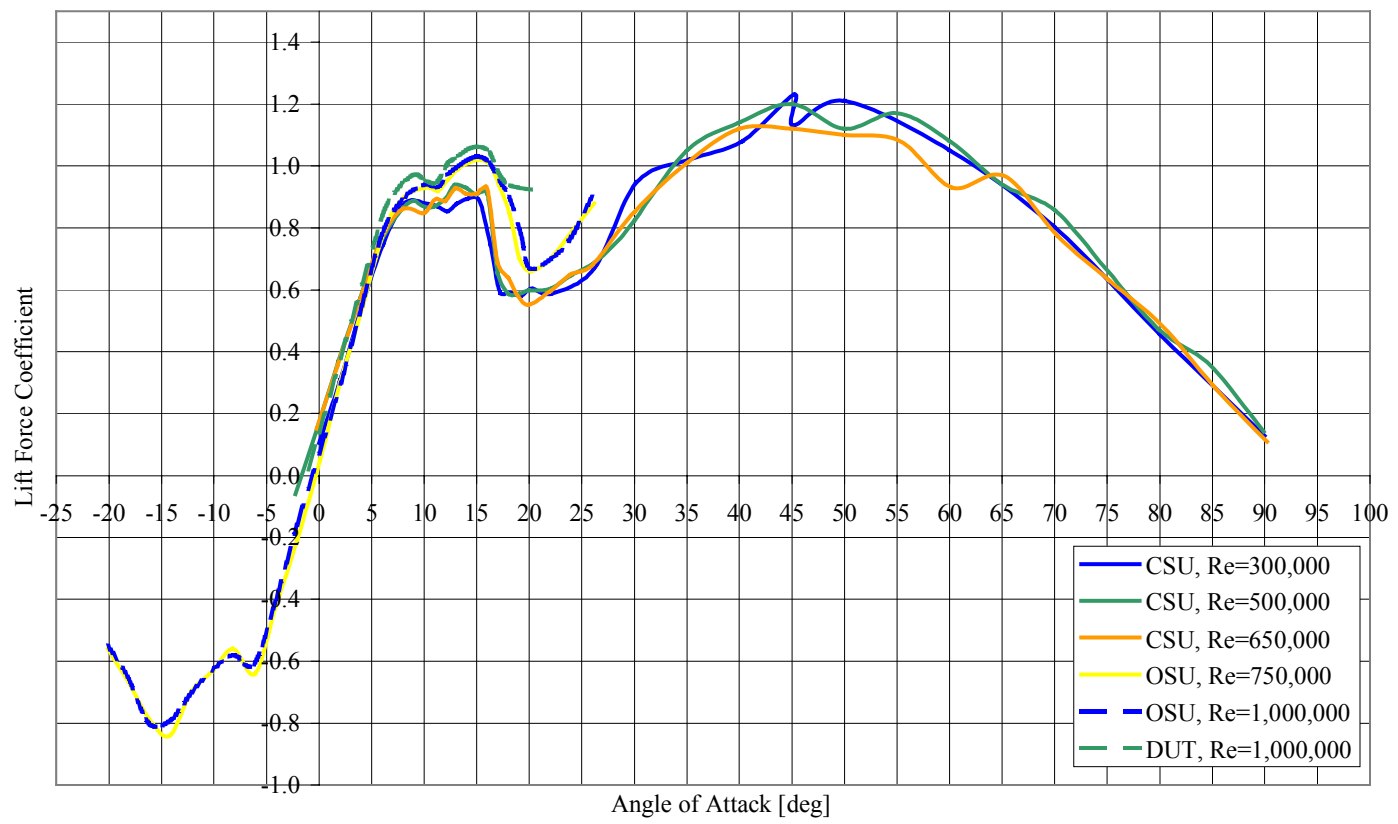

Figure 5.1: NREL S809 dimensionless lift force coefficient airfoil data 


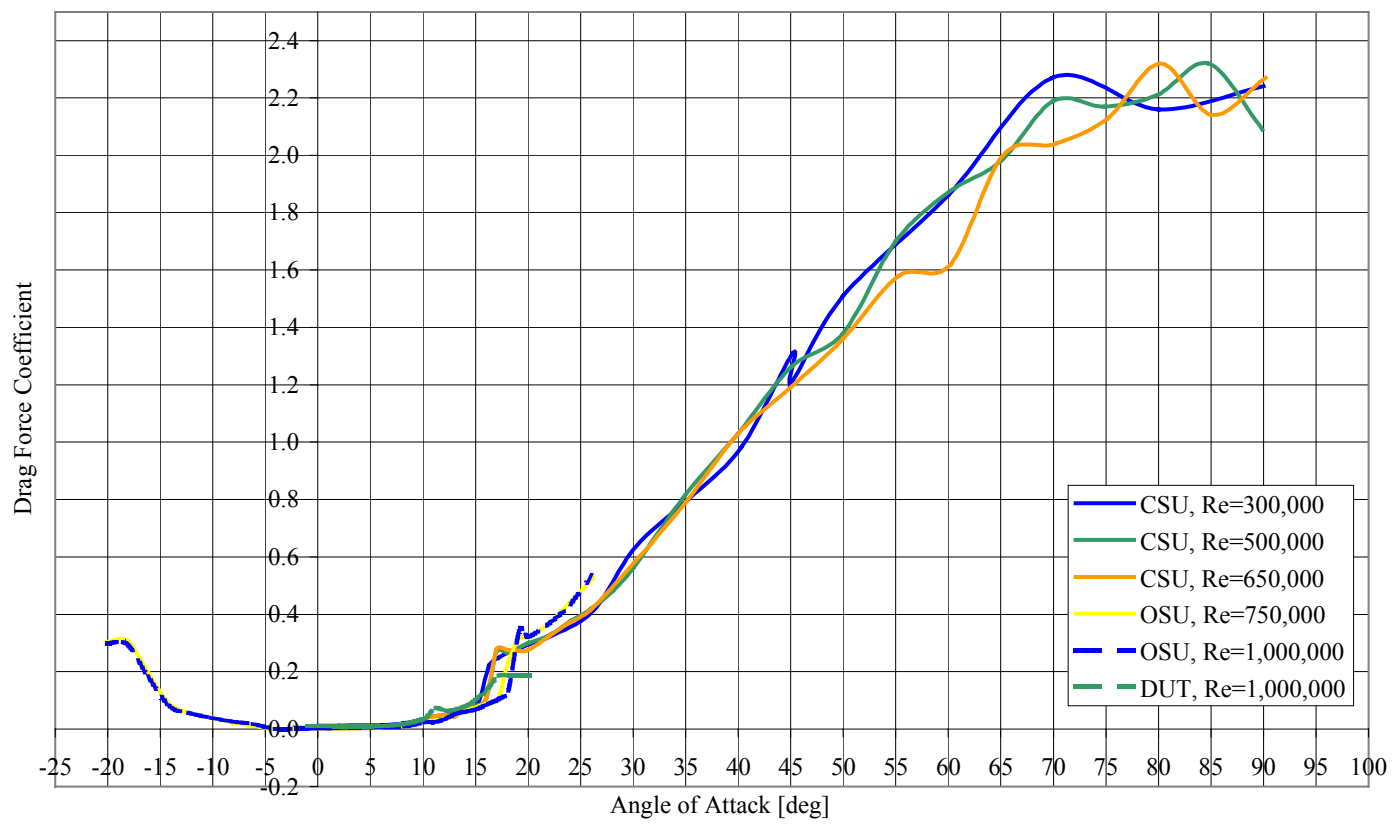

Figure 5.2: NREL S809 dimensionless drag force coefficient airfoil data

Structurally speaking, the rotor blades are manufactured with a fiberglass composite skin and a load-carrying unidirectional carbon fiber and $\pm 45^{\circ} \mathrm{S}$-glass fiber hybrid composite D-spar. Unfortunately, structural properties of the blades used on the UAE wind turbine are unavailable. The blade manufacturer, Composite Engineering, estimated mass and stiffness distributions for a similar blade: a constant $0.457 \mathrm{~m}$ chord, highly twisted blade, used during UAE Phase $\mathrm{V}^{24}$ testing. Data from Composite Engineering are listed in Table 5.8. These estimates were made using a computer model and include the effects of pressure measurement instrumentation and counterweights, as well as a root-mounted camera attachment.

\footnotetext{
${ }^{24}$ The UAE tests performed at the NASA-Ames Research Center in Moffett Field, California, encompass essentially Phase VI of a wind turbine analysis venture that has been going on since the early 1990s. Throughout the many phases of the project, several turbine configurations have been employed.
} 
Table 5.8: Structural Properties of the UAE Phase V Blades

\begin{tabular}{|l|l|r|r|r|r|}
\hline $\begin{array}{l}\text { Distance } \\
\text { from Rotor } \\
\text { Center }\end{array}$ & $\begin{array}{l}\text { Spanwise } \\
\text { Fraction } \\
(/ 5.029 \mathrm{~m})\end{array}$ & $\begin{array}{r}\text { Blade } \\
\text { Lineal } \\
\text { Density } \\
\left(\mu_{B}\right) \mathrm{kg} / \mathrm{m}\end{array}$ & $\begin{array}{r}\text { Local } \\
\text { Flapwise } \\
\text { Stiffness } \\
(E I) \mathrm{Nm}^{2}\end{array}$ & $\begin{array}{r}\text { Local } \\
\text { Edgewise } \\
\text { Stiffness } \\
(E I) \mathrm{Nm}^{2}\end{array}$ & $\begin{array}{r}\text { Local } \\
\text { Aerodynamic } \\
\text { Twist }\left(\theta_{T}\right) \\
\text { deg }\end{array}$ \\
\hline 0.402 & 0.080 & & 473,517 & 473,517 & 0.000 \\
\hline 0.508 & 0.101 & 30.14 & $2,320,700$ & $2,322,100$ & 0.000 \\
\hline 0.749 & 0.149 & 45.18 & $1,302,400$ & $1,556,800$ & 43.824 \\
\hline 1.006 & 0.200 & 46.09 & 710,230 & $1,332,800$ & 35.840 \\
\hline 1.257 & 0.250 & 31.53 & 471,680 & $1,165,553$ & 30.054 \\
\hline 1.509 & 0.300 & 16.92 & 232,180 & 997,640 & 24.246 \\
\hline 2.012 & 0.400 & 13.49 & 149,420 & 737,010 & 16.639 \\
\hline 3.515 & 0.500 & 12.95 & 123,480 & 650,900 & 11.431 \\
\hline 3.018 & 0.600 & 12.06 & 105,560 & 583,420 & 7.702 \\
\hline 4.520 & 0.700 & 11.19 & 84,468 & 512,420 & 4.922 \\
\hline 4.526 & 0.800 & 10.22 & 65,974 & 436,440 & 2.775 \\
\hline 5.029 & 1.000 & 9.25 & 46,953 & 387,600 & 1.070 \\
\hline
\end{tabular}

The differences between the UAE Phase V blades and the tapered, twisted blades used in the UAE Phase VI (i.e., NASA-Ames Research Center) tests are illustrated in Table 5.9.

Table 5.9: Differences between the UAE Phase V and Phase VI Blades

\begin{tabular}{|c|c|c|c|}
\hline Property & UAE Phase V Blade & UAE Phase VI Blade & Details \\
\hline Mass $[\mathrm{kg}]$ & 69.605 & 60.2 & Average value per blade \\
\hline $\begin{array}{l}\text { Root mounted camera } \\
\text { attached? }\end{array}$ & Yes & No & $\begin{array}{l}\text { The Phase } V \text { camera mass is } 13 \mathrm{~kg} \text {; the mass is equally } \\
\text { distributed between } 0.508 \text { and } 1.006 \mathrm{~m} \text { radius }\end{array}$ \\
\hline $\begin{array}{l}\text { Distance to c.g. from } \\
\text { center of rotation }[\mathrm{m}]\end{array}$ & 1.999 & 2.266 & Average value per blade \\
\hline Chord distribution & $\begin{array}{l}\text { Constant, } 0.457 \mathrm{~m} \text { beginning at } \\
0.723 \mathrm{~m} \text { from center of rotation }\end{array}$ & Tapered & This will affect the mass and stiffness distribution \\
\hline Twist distribution & $\begin{array}{l}\text { Ideal twist distribution (highly } \\
\text { twisted) }\end{array}$ & Slightly less twisted & $\begin{array}{l}\text { This will affect the out-of-plane and in-plane natural } \\
\text { frequency calculations }\end{array}$ \\
\hline $\begin{array}{l}1^{\text {st }} \text { in-plane natural } \\
\text { frequency }[\mathrm{Hz}]\end{array}$ & 8.065 & 8.981 & $\begin{array}{l}\text { Average value per blade relative to the condition when } \\
\text { the tip chord is parallel to the plane of rotation }\end{array}$ \\
\hline $\begin{array}{l}1^{\text {st }} \text { out-of-plane natural } \\
\text { frequency }[\mathrm{Hz}]\end{array}$ & 4.865 & 7.2815 & |" \\
\hline $\begin{array}{l}2^{\text {nd }} \text { out-of-plane natural } \\
\text { frequency }[\mathrm{Hz}]\end{array}$ & 20.7 & 29.75 & |" \\
\hline $\begin{array}{l}1^{\text {st }} \text { in-plane damping } \\
\text { ratio }\end{array}$ & $0.7650 \%$ & Data not available & Average value per blade \\
\hline $\begin{array}{l}1^{\text {st }} \text { out-of-plane } \\
\text { damping ratio }\end{array}$ & $0.9250 \%$ & Data not available & $"$ \\
\hline $\begin{array}{l}2^{\text {nd }} \text { out-of-plane } \\
\text { damping ratio }\end{array}$ & Data not available & Data not available & \\
\hline
\end{tabular}

Characteristics of the power train are:

- Power train inertia: $144-170 \mathrm{kgm}^{2}$

- Power train stiffness: 194,000-204,000 Nm/rad considering the low-speed shaft, gearbox, and high-speed shaft as one unit

- Power train natural frequency: $5.71-5.85 \mathrm{~Hz}$ 
- Power train damping ratio: $0.06-0.08$

- Gearbox ratio: 25.13 to 1

- Rated generator power: $20 \mathrm{~kW}$

- Synchronous speed of generator: $1,800 \mathrm{rpm}$ on the high-speed shaft side

- Generator slip: $1.69 \%$ at rated power

- Power train efficiency: about $78 \%$ including the combined effect of mechanical losses in the gearbox and mechanical and electrical losses in the generator

- Low-speed torque during startup: 2,210-2,261 Nm

- Maximum braking torque: $115 \mathrm{Nm}$

The tower is essentially a steel weldment assembly. A short and broad tubular steel lower section is connected to a long and narrow tubular steel upper section with a small conical segment. The base of the conical section is $3.4 \mathrm{~m}$ above the base of the tower and the top of the conical section is $3.9 \mathrm{~m}$ above the base of the tower. The bottom of the tower is connected to a circular base plate with 12 flanges. Other structural characteristics of the tower and nacelle include:

- Tower length: $11.5 \mathrm{~m}$

- Tower material: ASTM A106 schedule 40 and schedule 80 Type B pipe

- Tower Modulus of Elasticity: $200 \mathrm{GPa}$

- Tower shear Modulus: $77 \mathrm{GPa}$

- Tower density: $7,860 \mathrm{~kg} / \mathrm{m}^{3}$

- Tower base plate diameter/thickness: $1.829 \mathrm{~m} / 0.01905 \mathrm{~m} \mathrm{(3/4}$ inch)

- Base plate flange thickness: $0.01905 \mathrm{~m}(3 / 4$ inch)

- Base plate flange shape: $45^{\circ}-45^{\circ}-90^{\circ}$ triangle whose equal-length sides are $0.6097 \mathrm{~m}$ in length

- Lower tower outside diameter/wall thickness: $0.6096 \mathrm{~m} / 0.0175 \mathrm{~m}$

- Upper tower outside diameter/wall thickness: $0.4064 \mathrm{~m} / 0.0214 \mathrm{~m}$

- Tower mass: 3,317 kg including the effects of paint, welds, tower base plate, and 12 base plate flanges

- $1^{\text {st }}$ longitudinal/lateral natural frequency of tower alone: $3.91-4.03 \mathrm{~Hz}^{25}$

- $2^{\text {nd }}$ longitudinal/lateral natural frequency of tower alone: $19.50-19.91 \mathrm{~Hz}^{25}$

- Damping ratio associated with $1^{\text {st }}$ natural frequency of tower: $0.5-0.6^{25}$

- Damping ratio associated with $2^{\text {nd }}$ natural frequency of tower: $0.7-0.9^{25}$

- Nacelle mass: $1,712 \mathrm{~kg}$ including the mass of the hub, boom, and pitch shafts

- Nacelle inertia about yaw axis: $3,789 \mathrm{kgm}^{2}$ including the effects of the hub, boom, and pitch shafts

- Mass atop tower: $1,832 \mathrm{~kg}$ including the mass of the hub, boom, pitch shafts, and blades

- Inertia atop tower about yaw axis: approximately $4,640 \mathrm{kgm}^{2}$ including the effects of the hub, boom, pitch shafts, and blades

Because the UAE wind turbine was designed exclusively with research in mind, the UAE wind turbine is extensively instrumented. Bending moments are measured with strain gages installed on the low-speed shaft and at the root of each blade. In the wind tunnel tests, the rotor blade labeled "Blade 3" (out of two blades) was extensively instrumented and the structural properties of the other blade, "Blade 1," were balanced to match those of Blade 3. On Blade 3, the strain

\footnotetext{
${ }^{25}$ These values were obtained by examining modal test videos of the tower alone (see Hand 2001).
} 
gages measuring out-of-plane and in-plane moments at the root are applied to a steel pitch shaft adjacent to the blade attachment location and are aligned with the blade's tip chord line. The rotor torque is measured with additional strain gages installed on the low-speed shaft. A tension/compression load cell is used to measure moments about the yaw bearing. The yaw angle, azimuth angle of the instrumented blade (Blade 3), blade pitch angles, and blade flap angles were all measured with digital position encoders.

NREL has spent considerable time, energy, and money developing and perfecting instrumentation systems that can directly measure and characterize the aerodynamic forces acting on a rotating wind turbine blade. Aerodynamic coefficients for a particular radial station are found by integrating measured pressure values at that span location. To measure pressures, a grouping of 22 pressure taps, each connected to pressure transducer, is mounted chordwise around Blade 3. Each pressure tap opening is mounted flush to the airfoil surface to prevent the taps from disturbing the flow. The pressure transducers are mounted close to the pressure taps to mitigate potential dynamic effects within the tubes. These pressure tap groupings are installed at five primary spanwise locations: $30 \%, 46.67 \%, 63.33 \%, 80 \%$, and $95 \%$ span.

\section{Table 5.10: Aerodynamic Coefficient Pressure Tap Grouping Properties}

\begin{tabular}{|l|l|c|r|r|r|}
\hline $\begin{array}{l}\text { Distance from } \\
\text { Rotor Center } \\
m\end{array}$ & $\begin{array}{l}\text { Spanwise } \\
\text { Fraction } \\
(/ 5.029 \mathrm{~m})\end{array}$ & $\begin{array}{c}\text { Number Of } \\
\text { Pressure } \\
\text { Taps }\end{array}$ & $\begin{array}{r}\text { Local } \\
\text { Twist }\left(q_{T}\right) \\
\mathrm{deg}\end{array}$ & $\begin{array}{r}\text { Local Chord } \\
\text { Length }(c) \\
m\end{array}$ & $\begin{array}{r}\text { Local } \\
\text { Thickness } \\
m\end{array}$ \\
\hline 1.509 & 0.300 & 22 & 14.321 & 0.711 & 0.149 \\
\hline 2.347 & 0.467 & 22 & 4.692 & 0.627 & 0.131 \\
\hline 3.185 & 0.633 & 22 & 1.115 & 0.542 & 0.114 \\
\hline 4.023 & 0.800 & 22 & -0.381 & 0.457 & 0.096 \\
\hline 4.778 & 0.950 & 22 & -1.466 & 0.381 & 0.080 \\
\hline
\end{tabular}

Five-hole probes are used to measure local flow angles on the rotating blade. Like the pressure tap groupings, the probes are mounted at five primary spanwise locations, each located 4\% span outboard of its corresponding pressure tap grouping, except for the 95\% span station, where the probe is mounted 4\% span inboard. Angle of attack values at the pressure tap grouping span stations are derived from the local flow angle data by applying an upwash correction algorithm. Unfortunately, neither the probes nor the upwash correction algorithm are precise $\left( \pm 1^{\circ}\right.$ each).

Simms et al. (1999) comprehensively document information regarding the instrumentation systems used by the UAE research wind turbine.

\subsection{NASA-Ames Wind Tunnel Testing}

The UAE test program objectives summarized in the introduction of this chapter were met by testing the UAE research wind turbine in the world's largest wind tunnel located at the NASAAmes Research Center in Moffett Field (Silicon Valley), California. The so-called National Full-Scale Aerodynamics Complex wind tunnel has an enormous $24.4 \mathrm{~m}(80 \mathrm{ft})$ by $36.6 \mathrm{~m}(120$ $\mathrm{ft})$ test section, permitting the $10 \mathrm{~m}(33 \mathrm{ft})$ in diameter UAE wind turbine to be tested with minimal contribution of blockage effects. That is, the test section boundaries have a negligible influence on the induction of the stream tube passing through the plane of the rotor. 
Although the test section is enormous, flow through the wind tunnel is highly controlled and flow quality is consistently maintained. Flow is virtually steady, homogenous, uniform, and fixed in direction corresponding with assumptions (1) and (3) from section 2.2 and assumption (2) from section 2.3 . Flow speed deviates by no more than $0.25 \%$ across an empty test section, and the flow velocity vector diverges by no more than $0.5^{\circ}$ from the test section axis. Thus, testing in a highly controlled wind tunnel environment eliminates many of the complex characteristics of the natural, outdoor wind turbine environment- factors that complicate a detailed understanding of wind turbine aerodynamics.

To garner the most benefits associated with the wind tunnel tests, NREL formed a panel of advisers from around the world to guide in the selection of particular configurations and conditions under which the wind turbine should be operated in the wind tunnel. In the end, more than 1,700 tests, including variations in turbine configuration, pitch angles, yaw positions, and wind velocities, were conducted, resulting in almost any conceivable operation regime, including steady state, post stall, and dynamic stall. Throughout each test, the turbine was extensively instrumented to measure quantities such as inflow conditions, wake form, airfoil aerodynamic pressure distributions, and machine responses and loads. Certain measurements, such as angle of attack, were taken only during specific tests to thwart the obstruction of other measurements made on the wind turbine. Each test consisted of $30 \mathrm{~s}$ of data-recording time, corresponding to roughly 36 rotor revolutions.

Data obtained via the UAE wind tunnel tests are accurate and repeatable. The accuracies of the instrumentation systems are well documented by Simms et al. (1999). Exhaustive calibration procedures were performed between each wind tunnel test. During the wind tunnel tests, multiple independent data systems were also conducted to cross check incoming values, continuously ensuring high levels of data accuracy. Comparing the resulting data from similar tests demonstrates how astonishingly repeatable the data are, as data sets taken weeks apart are virtually identical. The acquired data represent the finest, most accurate set of wind turbine aerodynamic and induced flow field data available today. Simms et al. (2001) quantify additional information on data quality, accuracy, and repeatability.

\subsection{Blind Comparison Overview}

The key reasons for the Blind Comparison exercise were to (1) isolate deficiencies in the models employed in current design codes, (2) assess the range of applicability and efficiency of current design codes, and (3) obtain results that will guide the direction of future wind turbine modeling research. Thirty experts from 18 organizations around the world, representing an international conglomeration of industry, academic, and government wind turbine modeling specialists, participated and employed 19 wind turbine modeling tools. All participants were asked to provide documentation on their solution methodology, including information regarding model input parameters, other assumptions and approximations, theory employed in the models and the solution algorithm involved, computer performance, and post-processing tools.

The Blind Comparison exercise was executed to expose deficiencies linked with the modeling tools and the modelers' tactics. Shortcomings in the modeling tools originate from simulating operation states outside the scope of the models with which they were developed, flawed models, and code errors. Though all modelers were provided with the same turbine specifications (see 
section 5.1), the development of model input files is a discriminatory process that relies on modeler experience and intuition. For example, with the abundance of aerodynamic data provided (see Tables 5.2- 5.7 and Figs. 5.1 and 5.2), modelers must discern which data are most appropriate in each situation. The choice and method of extrapolating two-dimensional airfoil data to account for the effects of stall delay across the inboard stations of the blade (see the discussion of advanced aerodynamics topics in section 2.6) and deep stall is another example of modeler discernment. As demonstrated in Chapter 6, the selection of these input file parameters drastically affects the resulting predictions; thus, the Blind Comparison exercise also exposes deficiencies linked to the modelers' course of action.

Only 20 test cases were chosen for the Blind Comparison exercise, since a considerable amount of participant effort is required for each. Because the aerodynamic forces determine component loads and turbine performance, all the cases were chosen to target specific aerodynamic phenomena. The major differences between individual test cases were nominal wind speed and turbine yaw error. Nonzero yaw cases correspond to operating states in which the angle of attack is driven dynamically back and forth through static stall. Key parameters of each test scenario are listed in Table 5.11.

Table 5.11: Blind Comparison Test Scenarios

\begin{tabular}{|c|c|c|c|c|c|c|}
\hline Test Name & $\begin{array}{r}\text { Average } \\
\text { Wind } \\
\text { Speed } \\
m / s \\
\end{array}$ & $\begin{array}{r}\text { Yaw } \\
\text { Error } \\
\text { deg }\end{array}$ & $\begin{array}{r}\text { Average } \\
\text { Air } \\
\text { Density } \\
\mathrm{kg} / \mathrm{m}^{3}\end{array}$ & $\begin{array}{r}\text { Blade } \\
\text { Precone } \\
\text { Angle } \\
\text { deg }\end{array}$ & $\begin{array}{r}\text { Blade } \\
\text { Collective } \\
\text { Tip Pitch } \\
\text { Angle } \\
\text { deg } \\
\end{array}$ & $\begin{array}{r}\text { Average } \\
\text { Rotor } \\
\text { Rotational } \\
\text { Speed } \\
\text { rpm } \\
\end{array}$ \\
\hline \begin{tabular}{|l|} 
S0700000 \\
\end{tabular} & 7.0 & 0.0 & 1.246 & 0.0 & 3.0 & 71.9 \\
\hline S1000000 & 10.0 & 0.0 & 1.246 & 0.0 & 3.0 & 72.1 \\
\hline $\mathrm{S} 1300000$ & 13.1 & 0.0 & 1.227 & 0.0 & 3.0 & 72.1 \\
\hline \begin{tabular}{|l|}
$\mathrm{S} 1500000$ \\
\end{tabular} & 15.1 & 0.0 & 1.224 & 0.0 & 3.0 & 72.1 \\
\hline S2000000 & 20.1 & 0.1 & 1.221 & 0.0 & 3.0 & 72.0 \\
\hline S2500000 & 25.1 & 0.1 & 1.220 & 0.0 & 3.0 & 72.1 \\
\hline \begin{tabular}{|l|}
$\mathrm{S} 1000100$ \\
\end{tabular} & 10.1 & 10.0 & 1.246 & 0.0 & 3.0 & 72.1 \\
\hline \begin{tabular}{|l|}
$\mathrm{S} 1300100$ \\
\end{tabular} & 13.1 & 10.1 & 1.227 & 0.0 & 3.0 & 72.1 \\
\hline $\begin{array}{l}\mathrm{S} 1500100 \\
\end{array}$ & 15.1 & 10.0 & 1.224 & 0.0 & 3.0 & 72.1 \\
\hline $\mathrm{S} 1000300$ & 10.1 & 30.2 & 1.246 & 0.0 & 3.0 & 72.0 \\
\hline \begin{tabular}{|l|} 
S1300300 \\
\end{tabular} & 13.0 & 30.0 & 1.227 & 0.0 & 3.0 & 72.2 \\
\hline S1500300 & 15.1 & 29.9 & 1.225 & 0.0 & 3.0 & 72.2 \\
\hline \begin{tabular}{|l|} 
S1000600 \\
\end{tabular} & 10.1 & 60.0 & 1.246 & 0.0 & 3.0 & 71.7 \\
\hline \begin{tabular}{|l|} 
S1500600 \\
\end{tabular} & 15.1 & 60.0 & 1.225 & 0.0 & 3.0 & 71.9 \\
\hline E070000A & 7.0 & 0.1 & 1.234 & 3.4 & 3.0 & 71.9 \\
\hline E170000A & 17.1 & 0.1 & 1.234 & 3.4 & 3.0 & 72.0 \\
\hline E070020A & 7.1 & 20.0 & 1.234 & 3.4 & 3.0 & 71.9 \\
\hline E170020A & 17.2 & 20.2 & 1.234 & 3.4 & 3.0 & 72.2 \\
\hline E07M020A & 7.0 & -19.9 & 1.235 & 3.4 & 3.0 & 71.9 \\
\hline E17M020A & 17.2 & -20.0 & 1.234 & 3.4 & 3.0 & 72.1 \\
\hline
\end{tabular}

Measurement conventions were established to ensure continuity between modelers' predictions for the comparison phase, which was staged at the end of the Blind Comparison exercise. The Blind Comparison participants were asked to perform an equivalent simulation of each test case and to azimuth average the resulting time series data into $1^{\circ}$ azimuth angle bins (centered on the 
integer). The requested output channels and their associated measurement conventions are listed in Table 5.12. The requested data represent less than $1 \%$ of all data obtained during the UAE wind tunnel tests. 
Table 5.12: Blind Comparison Output Channels and Measurement Conventions

\begin{tabular}{|c|c|c|c|}
\hline $\begin{array}{l}\text { Channel } \\
\text { Name }\end{array}$ & Channel Description & $\begin{array}{c}\text { Channel } \\
\text { Units }\end{array}$ & $\begin{array}{l}\text { Channel } \\
\text { Measurement Convention }\end{array}$ \\
\hline$Y A W$ & Yaw error & deg & Positive clockwise looking down \\
\hline VTUN & Wind speed & $m / s$ & $\mathrm{~N} / \mathrm{A}$ \\
\hline$B 3 A Z I$ & Blade 3 azimuth angle & deg & $\begin{array}{l}\text { Counterclockwise when looking } \\
\text { downwind (for both upwind and } \\
\text { downwind configurations) }\end{array}$ \\
\hline$B 3 F L A P$ & Blade 3 coning (flap) angle & deg & Positive downwind \\
\hline B3PITCH & Blade 3 collective tip pitch angle & deg & Positive towards feather \\
\hline$R P M$ & Rotor rotational speed & rpm & $\mathrm{N} / \mathrm{A}$ \\
\hline$B 3 R F B$ & Blade 3 root flap bending moment & $\mathrm{Nm}$ & $\begin{array}{l}\text { Moment vector parallel to tip chord; } \\
\text { positive for downwind forces }\end{array}$ \\
\hline$B 3 R E B$ & Blade 3 root edge bending moment & $\mathrm{Nm}$ & $\begin{array}{l}\text { Moment vector perpendicular to tip } \\
\text { chord; positive in direction of rotation }\end{array}$ \\
\hline LSSTQ & Low speed shaft torque & $\mathrm{Nm}$ & Positive in direction of rotation \\
\hline$N A Y M$ & Nacelle yaw moment & $\mathrm{Nm}$ & $\begin{array}{l}\text { Positive to realign turbine in wind from } \\
\text { negative yaw error }\end{array}$ \\
\hline QNORM30 & Local dynamic pressure at $30.0 \%$ span & $P a$ & $\mathrm{~N} / \mathrm{A}$ \\
\hline QNORM47 & Local dynamic pressure at $46.7 \%$ span & $P a$ & $"$ \\
\hline QNORM63 & Local dynamic pressure at $63.3 \%$ span & $\mathrm{Pa}$ & " \\
\hline QNORM80 & Local dynamic pressure at $80.0 \%$ span & $P a$ & 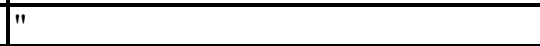 \\
\hline QNORM95 & Local dynamic pressure at $95.0 \%$ span & $P a$ & " \\
\hline CN30 & $30.0 \%$ span normal force coefficient & - & Standard definition \\
\hline CT30 & $30.0 \%$ span tangential force coefficient & - & $"$ \\
\hline$C M 30$ & $30.0 \%$ span pitching moment coefficient & - & $"$ \\
\hline CN47 & $46.7 \%$ span normal force coefficient & - & $"$ \\
\hline CT47 & $46.7 \%$ span tangential force coefficient & - & $"$ \\
\hline CM47 & $46.7 \%$ span pitching moment coefficient & - & $"$ \\
\hline CN63 & $63.3 \%$ span normal force coefficient & - & $"$ \\
\hline CT63 & $63.3 \%$ span tangential force coefficient & - & " \\
\hline CM63 & $63.3 \%$ span pitching moment coefficient & - & $"$ \\
\hline CN80 & $80.0 \%$ span normal force coefficient & - & $"$ \\
\hline CT80 & $80.0 \%$ span tangential force coefficient & - & $"$ \\
\hline CM80 & $80.0 \%$ span pitching moment coefficient & - & $"$ \\
\hline CN95 & $95.0 \%$ span normal force coefficient & - & 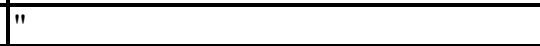 \\
\hline CT95 & $95.0 \%$ span tangential force coefficient & - & " \\
\hline CM95 & $95.0 \%$ span pitching moment coefficient & - & $"$ \\
\hline$N 30$ & $30.0 \%$ span normal force coefficient & N/unit span & Standard definition \\
\hline$T 30$ & $30.0 \%$ span tangential force coefficient & N/unit span & $"$ \\
\hline$M 30$ & $30.0 \%$ span pitching moment coefficient & Nm/unit span & $"$ \\
\hline N47 & $46.7 \%$ span normal force coefficient & N/unit span & $"$ \\
\hline T47 & $46.7 \%$ span tangential force coefficient & N/unit span & $"$ \\
\hline$M 47$ & $46.7 \%$ span pitching moment coefficient & Nm/unit span & $"$ \\
\hline N63 & $63.3 \%$ span normal force coefficient & N/unit span & $"$ \\
\hline T63 & $63.3 \%$ span tangential force coefficient & N/unit span & $"$ \\
\hline$M 63$ & $63.3 \%$ span pitching moment coefficient & Nm/unit span & $"$ \\
\hline$N 80$ & $80.0 \%$ span normal force coefficient & N/unit span & $"$ \\
\hline$T 80$ & $80.0 \%$ span tangential force coefficient & N/unit span & " \\
\hline$M 80$ & $80.0 \%$ span pitching moment coefficient & Nm/unit span & $"$ \\
\hline N95 & $95.0 \%$ span normal force coefficient & N/unit span & " \\
\hline T95 & $95.0 \%$ span tangential force coefficient & N/unit span & $"$ \\
\hline M95 & $95.0 \%$ span pitching moment coefficient & Nm/unit span & " \\
\hline
\end{tabular}




\section{Blind Comparison Input Parameters and Results}

FAST_AD has yet to be validated. Validation of wind turbine design codes such as FAST_AD involves comparing simulated load and performance predictions with actual experimental test data and assessing the degree to which they correspond. To facilitate validation of FAST_AD, the 20 test cases chosen for the Blind Comparison exercise are simulated. This permits a comparison between load and performance predictions made by FAST_AD and those obtained experimentally (permitting one to test how accurate the code predicts), and a comparison to load and performance predictions made by other design codes used worldwide (permitting one to test how FAST_AD's predictions compare to those of the wider wind turbine design and analysis community). The steps taken to perform simulations of the UAE research wind turbine under these scenarios and some of the results obtained are presented in this chapter. The steps taken to transform the UAE wind turbine specifications (summarized in Chapter 5) into specific input file entries are documented in section 6.1. A comparison and discussion of some of the load predictions made by FAST_AD and those obtained by other Blind Comparison participants and those obtained experimentally in the NASA-Ames wind tunnel are presented in section 6.2.

The goal of this chapter is not to determine whether the aerodynamic and structural models employed in FAST_AD are better than entirely dissimilar models employed by other design codes (which employ CFD, prescribed-wake, performance-only, or other solution algorithms). As a consequence, the results and discussion presented in section 6.2 are devoted entirely to the comparison of load and performance values obtained by means of the NASA-Ames wind tunnel tests, predictions made by FAST_AD, and predictions made only by Blind Comparison participants who employed the AeroDyn aerodynamics subroutines when determining aerodynamic forces acting on the rotor blades. All five Blind Comparison predictions that use the AeroDyn aerodynamics subroutines are presented. The reader is encouraged to examine Simms et al. (2001) for the results and conclusions of the entire Blind Comparison study. For anonymity, the load and performance predictions made by other Blind Comparison participants are labeled without names in section 6.2.

\subsection{FAST_AD Input Files Development for the Blind Comparison Study}

To transform the specifications of the UAE wind turbine presented in Chapter 5 into viable parameters needed to define the FAST_AD input file(s), several assumptions and approximations must be made. These assumptions and approximations, as well as other steps taken to develop the input file(s) for FAST_AD for the Blind Comparison test cases simulated, are documented in this section. An example input file is given in Appendix I for test case S0700000 of the Blind Comparison study of the UAE wind turbine. Input files for the other test cases are similar. Dissimilarities in the input files among the test cases are also discussed.

Following the practice of several Blind Comparison participants, an azimuth angle increment or azimuth step, $d \psi$, of about $1^{\circ}$ is used throughout all the simulations. In wind turbine analysis, reasoning in terms of an azimuth step, instead of a time step, is often convenient, though the main input file of FAST_AD requires the designation of a time step in seconds instead of degrees. The following simple formula can be applied to convert an azimuth step in degrees to a time step, $d t$, in seconds: 


$$
d t=\frac{d \psi\left(\frac{60 \mathrm{sec}}{1 \mathrm{~min}}\right)}{\Omega\left(\frac{360^{\circ}}{1 \mathrm{rev}}\right)}
$$

where the average angular speed of the rotor, $\Omega$, is assumed to be expressed in revolutions-perminute (rpm). Using an azimuth step of $1^{\circ}$ and an average rotor speed of $72 \mathrm{rpm}$, the time increment used for all simulations is $0.002 \mathrm{~s}$, rounded to the nearest thousandth of a second.

Each simulation is run for $30 \mathrm{~s}$, corresponding to roughly 36 revolutions of the rotor. To avoid recording data associated with the simulation startup transient $\mathrm{s}^{26}$, only data from the last $10 \mathrm{~s}$ of simulation time are recorded for data analysis purposes. Several test cases were run to verify that all startup transients die out before $20 \mathrm{~s}$ of simulation time pass.

All DOFs except yaw, tilt, and teeter are included (switched on) in all simulations. The yaw DOF is excluded to ensure that the yaw position of the nacelle and yaw error remain fixed throughout any individual simulation. The tilt DOF is excluded since information regarding the stiffness of the yaw bearing in the tilt direction is unavailable and since the yaw bearing is assumed to be stiff enough to prevent any substantial tilting motion of the nacelle. Since the UAE wind turbine exhibits a rigid rotor type for each Blind Comparison test case, the teeter DOF switch is also turned off.

FAST_AD considers the wind turbine modeled to spin clockwise when looking downwind and has the convention that a positive yaw angle is counterclockwise when looking downward for upwind and downwind rotor configurations. Since the sign conventions of both parameters are opposite to those used by the UAE wind turbine considered in the Blind Comparison test cases, equivalency is maintained and interpretation of proper sign conventions (of, for example, root bending moments) is not difficult. This is because the orientation of a blade advancing into a region of increased relative wind speed is identical in either perspective. The validity of this point is demonstrated in Fig. 6.1.

Since most blade load outputs available with FAST_AD are for Blade 1 only, Blade 1 in FAST_AD is used to model Blade 3 (the instrumented blade) of the UAE wind turbine. To ensure a proper azimuth angle convention, the azimuth offset angle, $z(4)$ is set fixed at $270^{\circ}$. The steady-state angular velocity of the rotor is defined to be the average rotor rotational speed listed in Table 5.11 for each Blind Comparison test scenario simulated. Similarly, the fixed yaw angle is set to the yaw error listed in Table 5.11 (these parameters are specified differently for each test case). All other initial conditions (initial wind turbine component displacements) are set equal to zero for each simulation run.

\footnotetext{
${ }^{26}$ The model starts out with initial displacements but zero initial inertias Once several seconds of the simulation have progressed, the inertias of most components of the turbine will no longer be zero and remain that way. The start-up transients are the events that must take place to make these inertias follow some quasi-steady-state pattern.
} 


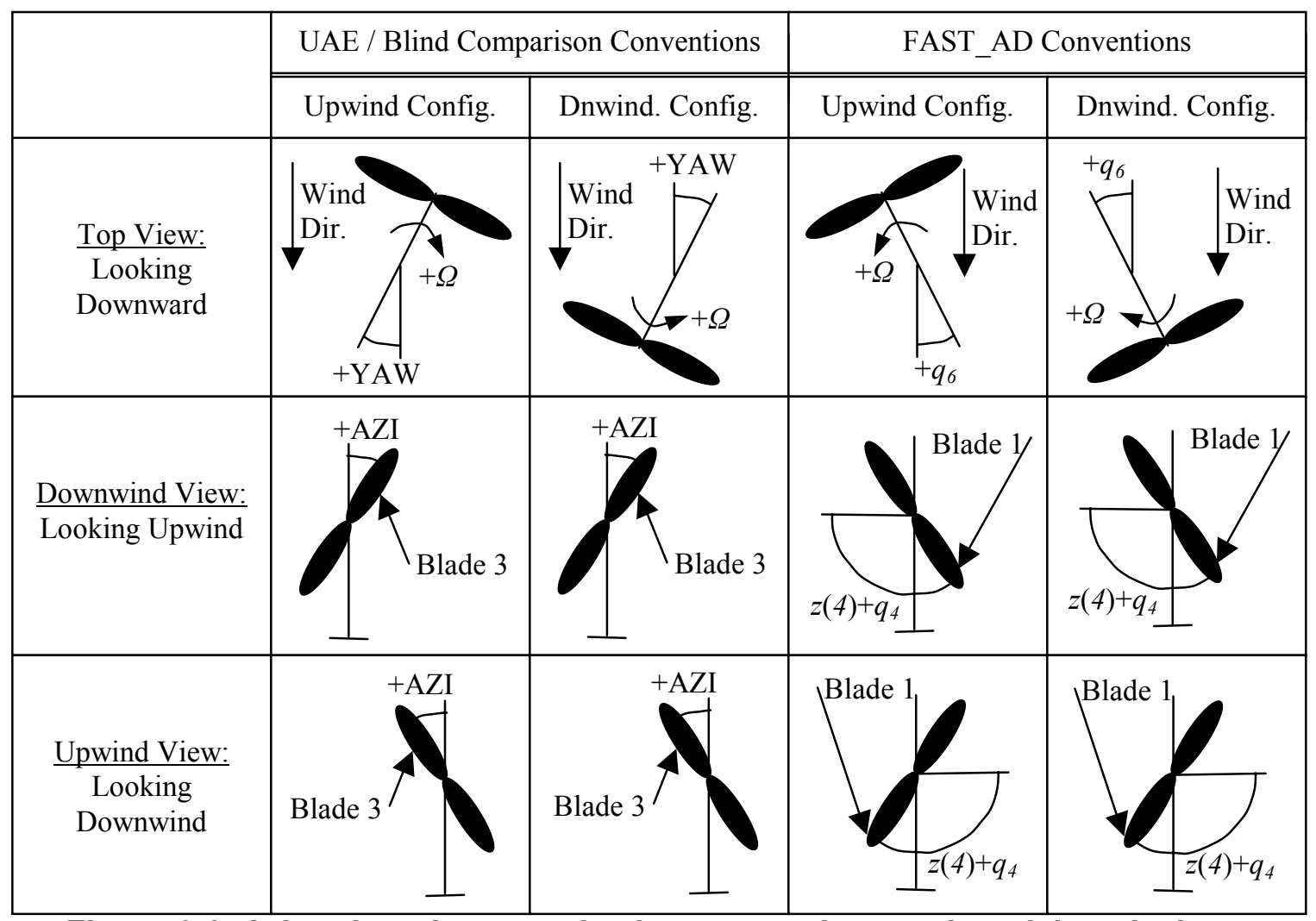

Figure 6.1: Azimuth and yaw angle sign conventions and model equivalency

The pitch mechanism is modeled as fixed (stall regulation). Since FAST_AD assumes that the blade collective pitch angle is measured relative to the point of zero aerodynamic twist and since the UAE wind turbine's tip twist is $-1.815^{\circ}$ relative to the point of zero aerodynamic twist at the $75 \%$ span location, the blade collective pitch angle is set at $4.815^{\circ}$ to ensure the angle to the tip chord line from the rotor plane is $3.0^{\circ}$ for all Blind Comparison test cases. The fixed blade precone angles, $\beta_{1}$ and $\beta_{2}$, are set to zero for the upwind rotor configurations and $3.4^{\circ}$ for the downwind rotor configurations as designated in Table 5.11.

Since the rotor is nonteetering, both the undersling length, $R_{U}$, and the delta-3 angle, $\delta_{3}$, are fixed at zero. The locations of the hub and nacelle center of masses are unknown; thus, the parameters $R_{U M}, D_{N M 1}$, and $D_{N M 2}$ are also set to zero. The designation of $R_{U M}=0$ is valid since blade coning angles are small or zero for each Blind Comparison test case; thus, the hub center of mass is most likely close to the blade axis intersection point. The designations of $D_{N M 1}=0$ and $D_{N M 2}=0$ are valid since the yaw and tilt DOFs are switched off and since the nacelle yaw moment is one of the noncrucial output channels, according to the directors of the Blind Comparison exercise. The distance to the teeter pin (or equivalently, to the blade axis intersection point since $R_{U M}$ is set to zero), $D_{N}$, is set to $-1.401 \mathrm{~m}$ for the upwind rotor configurations and $+1.401 \mathrm{~m}$ for the downwind rotor configurations.

Since inertial properties of the UAE wind turbine hub separate from the nacelle are not available and since the rotor is nonteetering, the hub is modeled as having no mass and its inertial effects are included with the inertial attributes of the nacelle. Consequently, the lumped mass of the 
nacelle is set at $1,712.0 \mathrm{~kg}$, the inertia of the nacelle about the yaw axis is set at $3,789.0 \mathrm{kgm}^{2}$, and the hub mass and inertia are set equal to zero.

The electric generator is modeled as an induction generator with a linear generator constant. The generator inertia is set at $161.5 \mathrm{kgm}^{2}$, the average value from the range provided (144-170 $\mathrm{kgm}^{2}$ ). FAST_AD permits the designation of independent gearbox and generator efficiencies. Since only an effective power train efficiency of the UAE wind turbine is provided $(78 \%)$, the gearbox efficiency is set at $100 \%$ and the generator efficiency is set at $78 \%$. The synchronous speed of the generator (zero-slip condition) is $1,800 \mathrm{rpm}$ on the high-speed shaft side. This speed must be designated in the FAST_AD input file relative to the low-speed shaft $\left(\Omega_{0}\right)$. Since the gearbox ratio is $25.13: 1, \Omega_{0}$ is set at $71.63 \mathrm{rpm}$. Likewise, the rated speed of the induction generator must be specified relative to the low-speed shaft $\left(\Omega_{R}\right)$. The rated speed, synchronous speed, and slip of a linearly modeled induction generator are related:

$$
\text { generator slip at rated power }=\frac{\Omega_{R}-\Omega_{0}}{\Omega_{0}}
$$

With a generator slip at rated power of $1.69 \%$ for the UAE wind turbine, the rated speed of the induction generator relative to the low-speed shaft, $\Omega_{R}$, is set at $72.82 \mathrm{rpm}$. The linear generator constant, CINGEN, is equal to the low-speed shaft torque at rated power, $L S S T Q_{R}$, divided by the product of the gearbox ratio squared, $Y N^{2}$, and the difference in rated and synchronous speeds relative to the low-speed shaft measured in radians-per-second:

$$
C I N G E N=\frac{\operatorname{SSTT}_{R}\left(\frac{60 \mathrm{sec}}{1 \mathrm{~min}}\right)}{Y N^{2}\left(\Omega_{R}-\Omega_{0}\right)\left(\frac{2 \pi \mathrm{rad}}{1 \mathrm{rev}}\right)}
$$

The low-speed shaft torque at rated power, $L S S T Q_{R}$, is the ratio of the induction generator's rated power, $P_{R}$, and the rated speed of the low-speed shaft measured in radians-per-second:

$$
L S S T Q_{R}=\frac{P_{R}\left(\frac{60 \mathrm{sec}}{1 \mathrm{~min}}\right)}{\Omega_{R}\left(\frac{2 \pi \mathrm{rad}}{1 \mathrm{rev}}\right)}
$$

With a rated generator power of $20 \mathrm{~kW}(20,000 \mathrm{~W}), L S S T Q_{R}$ is 2,622 $\mathrm{Nm}$ and CINGEN is set at $32.7539 \mathrm{Nm} /(\mathrm{rad} / \mathrm{s})$. Though not needed in any simulations, the high-speed shaft startup torque is set at $88.9574 \mathrm{Nm}$ in the FAST_AD input file. This is the average value of the startup lowspeed shaft torque divided by gearbox ratio from the range of startup low-speed shaft torques provided $(2,210-2,261 \mathrm{Nm})$. 
Flexibility of the drive shaft is modeled with a linear torsional spring and a linear viscous damper, both specified as constants in the FAST_AD input file. The equivalent drive train torsional spring constant, $Z K D R V$, is set at $199,000 \mathrm{Nm} / \mathrm{rad}$, the average value from the range provided (194,000-204,000 Nm/rad). The equivalent drive train torsional damper constant, $C D R V$, is equal to twice the product of $Z K D R V$ and the power train damping ratio, $\zeta_{D R V}$, divided by the power train natural frequency in radians-per-second, $\omega_{n D R V}$ :

$$
C D R V=2 \frac{Z K D R V \cdot \zeta_{D R V}}{\omega_{n D R V}}
$$

The average power train damping ratio from the range provided $(0.06-0.08)$ is 0.07 . The average power train natural frequency from the range provided $(5.71-5.85 \mathrm{~Hz})$ is $5.78 \mathrm{~Hz}$, or $36.3168 \mathrm{rad} / \mathrm{s}$. Thus, $C D R V$ is approximated as $767.138 \mathrm{Nm} /(\mathrm{rad} / \mathrm{s})$.

The entire length of the tower $(11.5 \mathrm{~m})$ is modeled as flexible. The rigid length of the tower, $H_{S}$, is thus set to zero. The distance from the tower top base plate (yaw bearing) to the rotor axis, TWRHTOFFSET, is set at $0.692 \mathrm{~m}$, the difference between the hub height above ground level $(12.192 \mathrm{~m})$ and the length of the tower $(11.5 \mathrm{~m})$.

Several approximations are made to estimate the structural properties of the tower and its corresponding natural mode shapes. To estimate the distributed lineal density of the tower, $\mu_{T}(h)$, estimates of masses of various parts of the tower are made and scaled so their sum, the total approximated tower mass, is equal to the actual measured tower mass $(3,317 \mathrm{~kg})$. First, the mass of several sections of the tower (upper segment, lower segment, conical segment, base plate, and flanges) are estimated, summed, and compared to the total tower mass provided for the UAE wind turbine. The details of each calculation follow without explanation, but should be easily inferred from the data given in Chapter 5:

$$
\begin{aligned}
& \begin{aligned}
\mu_{T}(\text { upper segment }) & =\left(7,860 \frac{\mathrm{kg}}{\mathrm{m}^{3}}\right)\left\{\pi\left[\left(\frac{0.4064 \mathrm{~m}}{2}\right)^{2}-\left(\frac{0.4064 \mathrm{~m}}{2}-0.0214 \mathrm{~m}\right)^{2}\right]\right\} \\
& =203.445 \frac{\mathrm{kg}}{\mathrm{m}}
\end{aligned} \\
& \begin{aligned}
\mu_{T}(\text { lower segment }) & =\left(7,860 \frac{\mathrm{kg}}{\mathrm{m}^{3}}\right)\left\{\pi \text { of upper tower segment }=\left(203.445 \frac{\mathrm{kg}}{\mathrm{m}}\right)(11.5 \mathrm{~m}-3.9 \mathrm{~m})=1,546.182 \mathrm{~kg}\right. \\
& =255.862 \frac{\mathrm{kg}}{\mathrm{m}}
\end{aligned}
\end{aligned}
$$




$$
\begin{gathered}
\text { mass of lower tower segment }=\left(255.862 \frac{\mathrm{kg}}{\mathrm{m}}\right)(3.4 \mathrm{~m})=869.931 \mathrm{~kg} \\
\text { mass of conical tower segment }=\frac{1}{2}\left(203.445 \frac{\mathrm{kg}}{\mathrm{m}}+255.862 \frac{\mathrm{kg}}{\mathrm{m}}\right)(3.9 \mathrm{~m}-3.4 \mathrm{~m}) \\
=114.827 \mathrm{~kg} \\
\text { mass of base plate }=\left(7,860 \frac{\mathrm{kg}}{\mathrm{m}^{3}}\right)\left[\pi\left(\frac{1.829 \mathrm{~m}}{2}\right)^{2}(0.01905 \mathrm{~m})\right]=393.400 \mathrm{~kg} \\
\text { mass of } \text { sin gle flange }=\left(7,860 \frac{\mathrm{kg}}{\mathrm{m}^{3}}\right)\left[\frac{1}{2}(0.6097 \mathrm{~m})^{2}(0.01905 \mathrm{~m})\right]=27.830 \mathrm{~kg} \\
\text { and }
\end{gathered}
$$$$
\text { total tower mass }=1,546.182 \mathrm{~kg}+869.931 \mathrm{~kg}+114.827 \mathrm{~kg}+393.400 \mathrm{~kg}
$$$$
+(12)(27.830 \mathrm{~kg})=3,258.305 \mathrm{~kg}
$$

The estimated total tower mass given in Eq. (6.13) is $1.80 \%$ less than the total tower mass measured. The difference is most likely due to the addition of paint and welds in the latter. Consequently, the lineal densities of the upper and lower tower segments calculated above are scaled up by a factor of $1.018-207.107 \mathrm{~kg} / \mathrm{m}$ and $260.467 \mathrm{~kg} / \mathrm{m}$ respectively. These calibrated distributed lineal density parameters are used during calculations of the tower's mode shapes in program Modes and specification of these tower properties in the input file of FAST_AD.

Since the mass distribution is already calibrated and since the stiffness distribution, $E I_{T}(h)$, is the only other set of tower parameters that is not well characterized, estimates of the stiffness distribution are calibrated to ensure that the first natural frequency predicted by Modes of the tower alone is equal to $3.97 \mathrm{~Hz}$, the average value from the range provided $(3.91-4.03 \mathrm{~Hz})$. A preliminary estimate of the distributed stiffness of the tower is made by estimating the stiffness of the upper and lower tower segments (again, the details of each calculation follow without explanation but should be easily inferred from the data given in Chapter 5):

$$
\begin{aligned}
E I_{T}(\text { upper segment }) & =(200 \mathrm{GPa})\left\{\frac{\pi}{4}\left[\left(\frac{0.4064 \mathrm{~m}}{2}\right)^{4}-\left(\frac{0.4064 \mathrm{~m}}{2}-0.0214 \mathrm{~m}\right)^{4}\right]\right\} \\
& =96,211,189.2 \mathrm{Nm}^{2}
\end{aligned}
$$




$$
\begin{aligned}
E I_{T}(\text { lower segment }) & =(200 \mathrm{GPa})\left\{\frac{\pi}{4}\left[\left(\frac{0.6096 \mathrm{~m}}{2}\right)^{4}-\left(\frac{0.6096 \mathrm{~m}}{2}-0.0175 \mathrm{~m}\right)^{4}\right]\right\} \\
& =285,556,680.0 \mathrm{Nm}^{2}
\end{aligned}
$$

To calibrate this stiffness distribution, the first two natural frequencies of the tower alone are predicted by program Modes and compared to the natural frequencies of the tower specified in Chapter 5. This is done by specifying the parameters into a Modes input file (reference Appendix II for layout) and running the program with the end mass, $M_{\text {Top }}$, equal to zero. The resulting frequency predictions are higher than those specified in Chapter 5. Both distributed stiffness values must be scaled by a factor of 0.826 to achieve equivalency of the first natural frequency. The resulting calibrated stiffness is $79,470,442.3 \mathrm{Nm}^{2}$ for the upper tower section and $235,869,817.7 \mathrm{Nm}^{2}$ for the lower tower section. The resulting predicted second natural frequency is $19.898 \mathrm{~Hz}$, which is within the range of the values provided $(19.50-19.91 \mathrm{~Hz})$.

To obtain the proper mode shapes needed for the FAST_AD input file (tower mode shapes that take into account the mass atop the tower), the tower end mass is specified to be the combined mass of the nacelle and rotor $(1,832 \mathrm{~kg})$ in the Modes input file and Modes is run again. A summary of the calibrated distributed tower parameters and the resulting mode shapes is given in Tables 6.1 and 6.2.

Table 6.1: Distributed Properties of the UAE Tower

\begin{tabular}{|l|l|r|r|}
\hline $\begin{array}{l}\text { Ht. Above } \\
\text { Ground }(h) \\
m\end{array}$ & $\begin{array}{l}\text { Fractional } \\
\text { Distance } \\
(h / 11.5 \mathrm{~m})\end{array}$ & $\begin{array}{r}\text { Lineal } \\
\text { Density } \\
\left(\mu_{T}\right) \mathrm{kg} / \mathrm{m}\end{array}$ & $\begin{array}{r}\text { Tower } \\
\text { Stiffness } \\
\left(E I_{T}\right) \mathrm{Nm}^{2}\end{array}$ \\
\hline 0.000 & 0.00000 & 260.467 & $235,869,817.7$ \\
\hline 3.400 & 0.29565 & 260.467 & $235,869,817.7$ \\
\hline 3.401 & 0.29574 & 260.467 & $235,869,817.7$ \\
\hline 3.899 & 0.33904 & 207.107 & $79,470,442.3$ \\
\hline 3.900 & 0.33913 & 207.107 & $79,470,442.3$ \\
\hline 11.500 & 1.00000 & 207.107 & $79,470,442.3$ \\
\hline
\end{tabular}

Table 6.2: Mode Shapes of the UAE Tower (Including Mass atop Tower)

\begin{tabular}{|c|c|r|r|}
\hline $\begin{array}{c}\text { Shape } \\
\text { Function } \\
\text { Number }(b)\end{array}$ & $\begin{array}{c}\text { Shape } \\
\text { Function } \\
\left(\varphi_{b}{ }^{\prime}\right)^{\prime}\end{array}$ & $\begin{array}{r}1^{\text {st }} \text { Mode Const. } \\
\text { Proportionality } \\
\text { Coef.s }\left(C_{1, b}{ }^{\prime}\right)\end{array}$ & $\begin{array}{r}2^{\text {nd }} \text { Mode Const. } \\
\text { Proportionality } \\
\text { Coef.s }\left(C_{2, b}{ }^{\prime}\right)\end{array}$ \\
\hline 2 & $(h / H)^{2}$ & 1.187 & -29.592 \\
\hline 3 & $(h / H)^{3}$ & -2.497 & -1.335 \\
\hline 4 & $(h / H)^{4}$ & 6.824 & 85.063 \\
\hline 5 & $(h / H)^{5}$ & -6.654 & -62.876 \\
\hline 6 & $(h / H)^{6}$ & 2.140 & 9.740 \\
\hline
\end{tabular}

The modal damping ratio associated with the first natural frequency of the tower is set at 0.55 , the average value from the range provided (0.5-0.6). The modal damping ratio associated with 
the second natural frequency of the tower is set at 0.8 , again the average value from the range provided (0.7-0.9). The maximum number of constant length increments along the tower allowable for integration of elastic forces is chosen (20).

Though never used because the yaw DOF is switched off, parameters associated with flexibility of the yaw drive in the yaw direction are specified in the FAST_AD input file as accurately as possible. Since the yaw bearing is very stiff, the stiffness of the yaw drive in the yaw direction is equal to the torsional stiffness of the tower. To determine this stiffness, the torsional stiffness of the upper and lower tower segments are first estimated as follows ${ }^{27}$ (again, the details of each calculation follow without explanation but should be easily inferred from the data given in Chapter 5):

$$
\begin{aligned}
& \text { torsional stiffness of upper segment }= \\
& \qquad \frac{(77 \mathrm{GPa})}{(11.5 \mathrm{~m}-3.9 \mathrm{~m})}\left\{\frac{\pi}{2}\left[\left(\frac{0.4064 \mathrm{~m}}{2}\right)^{4}-\left(\frac{0.4064 \mathrm{~m}}{2}-0.0214 \mathrm{~m}\right)^{4}\right]\right\} \\
& =9,747,713 \frac{\mathrm{Nm}}{\mathrm{rad}}
\end{aligned}
$$

and

torsional stiffness of lower segment $=$

$$
\frac{(77 \mathrm{GPa})}{(3.4 \mathrm{~m})}\left\{\frac{\pi}{2}\left[\left(\frac{0.6096 \mathrm{~m}}{2}\right)^{4}-\left(\frac{0.6096 \mathrm{~m}}{2}-0.0175 \mathrm{~m}\right)^{4}\right]\right\}=64,670,189 \frac{\mathrm{Nm}}{\mathrm{rad}}
$$

The stiffness of the upper and lower tower segments combine in series to determine the equivalent torsional yaw spring constant, $Z K Y A W^{28}$ :

$$
Z K Y A W=\frac{\left(9,747,713 \frac{\mathrm{Nm}}{\mathrm{rad}}\right)\left(64,670,189 \frac{\mathrm{Nm}}{\mathrm{rad}}\right)}{9,747,713 \frac{\mathrm{Nm}}{\mathrm{rad}}+64,670,189 \frac{\mathrm{Nm}}{\mathrm{rad}}}=8,471,000 \frac{\mathrm{Nm}}{\mathrm{rad}}
$$

27 The torsional stiffness of an linearly elastic, isotropic, and homogenous rod is defined as:

$$
k=\frac{G J}{L}
$$

${ }^{28}$ The effective stiffness of set of two linearly elastic springs in series is:

$$
k_{e f f}=\frac{k_{1} k_{2}}{k_{1}+k_{2}}
$$


Similarly, the torsional damping constant of the yaw drive in the yaw direction, $Z C Y A W$, is equal to the torsional damping of the tower. Assuming a damping ratio of $2 \%, Z C Y A W$ is estimated ${ }^{29}$ :

$$
Z C Y A W=2(0.02) \sqrt{\left(8,471,000 \frac{\mathrm{Nm}}{\mathrm{rad}}\right)\left(4,640 \mathrm{kgm}^{2}\right)}=7,930 \frac{\mathrm{Nm}}{\mathrm{rad} / \mathrm{sec}}
$$

The hub radius, or equivalently, the rigid blade length, $R_{H}$, is set at $0.432 \mathrm{~m}$, the distance from the hub centerline to the root-mounted strain gages. $0.432 \mathrm{~m}$ is chosen instead of $0.508 \mathrm{~m}$, the actual hub radius, to ensure that the blade root stresses are characterized correctly. The length of the flexible part of the blade is thus designated as $4.597 \mathrm{~m}(5.029 \mathrm{~m}$ rotor radius minus $0.432 \mathrm{~m}$ hub radius).

Like the tower, several approximations are made to estimate the structural properties of the blades and their corresponding natural mode shapes. Estimations of the structural properties of the blades are more difficult, however, since detailed information on the blades used during the NASA-Ames wind tunnel tests is unavailable. The flapwise and edgewise stiffness distributions listed in Table 5.8 are for the blades of the wind turbine used during Phase V testing and the differences between these blades and those used during the NASA-Ames wind tunnel tests (UAE Phase VI testing) are listed in Table 5.9. Also, the natural frequencies listed in Table 5.9 are outof-plane and in-plane frequencies relative to the condition in which the tip chord is parallel to the rotor plane. Program Modes allows its user to input a structural pretwist distribution so the natural frequencies can be found in the out-of-plane and in-plane directions, allowing the user to match physically measured frequencies. Once such frequencies are matched, the distributed structural pretwist must be zeroed out to obtain the proper mode shapes needed for the FAST_AD input file.

With this in mind, the first step to obtain structural properties of the UAE Phase VI blades and their corresponding mode shapes is to adjust the mass and stiffness distributions of the Phase V turbine so that program Modes accurately predicts the experimentally measured out-of-plane and in-plane frequencies given in Table 5.9. After adequate information on the mass and stiffness distributions for the Phase $\mathrm{V}$ blades is in-hand, these parameters are modified to account for the differences between the UAE Phase V and Phase VI blades listed in Table 5.9. Finally, the stiffness distributions are calibrated by ensuring that the natural frequencies of the UAE Phase VI blades predicted by Modes are equivalent to those corresponding frequencies also listed in Table 5.9.

To calibrate the mass and stiffness distributions of the Phase V blades, similar steps are taken to those used to calibrate the mass and stiffness distributions of the tower. The mass distribution of the Phase $\mathrm{V}$ turbine is first tuned so a computation of the blade mass is equal to the actual measured blade mass $(69.605 \mathrm{~kg})$. The blade mass is estimated with the data provided in Table 5.8 by dividing the blade into a number of elements, calculating their masses, and summing.

${ }^{29}$ The torsional damping constant for a linearly elastic, isotropic, and homogenous rod is defined as:

$$
c=2 \zeta \sqrt{k J}
$$


This is done by using elements that are centered at the radial distances listed in Table 5.8 (the nodes between the elements are located halfway between these radial distances and the elements at the endpoints are only half-elements) as illustrated in Table 6.3. Each element's contribution to the center of gravity (c.g.) of the blade is found by multiplying the element's mass by the radial distance to its center and dividing by the total blade mass.

Table 6.3: Computation of the UAE Phase V Blade Mass

\begin{tabular}{|c|c|c|c|c|}
\hline \multirow{4}{*}{\begin{tabular}{|l} 
Distance \\
from Rotor \\
Center \\
$m$ \\
\end{tabular}} & \multirow{4}{*}{$\begin{array}{r}\text { Blade } \\
\text { Lineal } \\
\text { Density } \\
\left(\mu_{B}\right) \mathrm{kg} / \mathrm{m} \\
\end{array}$} & \multicolumn{3}{|c|}{ Properties of an Element Centered at $r$} \\
\hline & & Element & Element & Element \\
\hline & & Length & Mass & Contribution \\
\hline & & $m$ & $\mathrm{~kg}$ & to c.g. $m$ \\
\hline \multicolumn{5}{|l|}{0.402} \\
\hline 0.508 & 30.14 & 0.121 & 3.632 & 0.024 \\
\hline 0.749 & 45.18 & 0.249 & 11.250 & 0.108 \\
\hline 1.006 & 46.09 & 0.254 & 11.707 & 0.151 \\
\hline 1.257 & 31.53 & 0.252 & 7.931 & 0.128 \\
\hline 1.509 & 16.92 & 0.378 & 6.387 & 0.124 \\
\hline 2.012 & 13.49 & 0.503 & 6.785 & 0.175 \\
\hline 2.515 & 12.95 & 0.503 & 6.514 & 0.210 \\
\hline 3.018 & 12.06 & 0.503 & 6.060 & 0.234 \\
\hline 3.520 & 11.19 & 0.503 & 5.623 & 0.254 \\
\hline 4.023 & 10.22 & 0.503 & 5.141 & 0.265 \\
\hline 4.526 & 9.25 & 0.503 & 4.653 & 0.270 \\
\hline 5.029 & 9.32 & 0.252 & 2.344 & 0.151 \\
\hline TOTALS & & 4.521 & 78.026 & 2.093 \\
\hline
\end{tabular}

The estimated blade mass given in Table 6.3 is considerably larger than that measured and listed in Table 5.9. A scaling factor of 0.892 applied to the distributed lineal density of the blades, $\mu_{B}(r)$, is required to calibrate the two masses. Since the predicted blade c.g. of $2.093 \mathrm{~m}$ from the center of rotation is close to the value of $1.999 \mathrm{~m}$ listed in Table 5.9 without any modification to the mass distribution, this is purely a magnitude adjustment.

Now that a calibrated distributed lineal density of the Phase V blades is acquired, the magnitudes of the flapwise and edgewise stiffness distributions of the Phase V turbine blades (listed in Table 5.8) are scaled (calibrated) so the out-of-plane and in-plane natural frequencies predicted by program Modes match those given in Table 5.9 (approximately 4.865 and $8.065 \mathrm{~Hz}$ for the first natural modes, respectively). Magnitude scaling factors of 0.72 and 0.83 applied to the flapwise and edgewise stiffness distributions are necessary to achieve equivalency of these first natural frequencies. Since the actual structural pretwist distribution is unknown, it is assumed to coincide with the aerodynamic twist distribution during this process. Also, the end mass, $M_{\text {Tip }}$, is set equal to zero in this process because there are no tip brakes. Centrifugal stiffening effects are not accounted for in this process. A summary of the calibrated distributed structural properties of the UAE Phase V blades is given in Table 6.4. 
Table 6.4: Calibrated Properties of the UAE Phase V Blades

\begin{tabular}{|c|c|c|c|c|c|c|}
\hline \begin{tabular}{|l} 
Distance \\
from Rotor \\
Center \\
$m$ \\
\end{tabular} & \begin{tabular}{|l|} 
Distance \\
Along Blade \\
From Root $(r)$ \\
$m$
\end{tabular} & \begin{tabular}{|l|} 
Radial \\
Fraction \\
$(r / 4.597 \mathrm{~m})$ \\
\end{tabular} & $\begin{array}{r}\text { Blade } \\
\text { Lineal } \\
\text { Density } \\
\left(\mu_{B}\right) \mathrm{kg} / \mathrm{m} \\
\end{array}$ & $\begin{array}{r}\text { Local } \\
\text { Flapwise } \\
\text { Stiffness } \\
(E I) \mathrm{Nm}^{2} \\
\end{array}$ & $\begin{array}{r}\text { Local } \\
\text { Edgewise } \\
\text { Stiffness } \\
(E I) \mathrm{Nm}^{2} \\
\end{array}$ & $\begin{array}{r}\text { Local } \\
\text { Structural } \\
\text { Twist }\left(\theta_{S}\right) \\
d e g \\
\end{array}$ \\
\hline 0.432 & 0.000 & 0.00000 & 0.000 & $340,932.2$ & $393,019.1$ & 0.000 \\
\hline 0.508 & 0.076 & 0.01653 & 26.885 & $1,670,904.0$ & $1,927,343.0$ & 0.000 \\
\hline 0.749 & 0.317 & 0.06896 & 40.301 & $937,728.0$ & $1,292,144.0$ & 43.824 \\
\hline 1.006 & 0.574 & 0.12486 & 41.112 & $511,365.6$ & $1,106,224.0$ & 35.840 \\
\hline 1.257 & 0.825 & 0.17946 & 28.128 & $339,609.7$ & $967,409.1$ & 30.054 \\
\hline 1.509 & 1.077 & 0.23428 & 15.093 & $167,169.6$ & $828,041.2$ & 24.246 \\
\hline 2.012 & 1.580 & 0.34370 & 12.033 & $107,582.4$ & $611,718.3$ & 16.639 \\
\hline 2.515 & 2.083 & 0.45312 & 11.551 & $88,905.6$ & $540,247.0$ & 11.431 \\
\hline 3.018 & 2.586 & 0.56254 & 10.758 & $76,003.2$ & $484,238.6$ & 7.702 \\
\hline 3.520 & 3.088 & 0.67174 & 9.981 & $60,817.0$ & $425,308.6$ & 4.922 \\
\hline 4.023 & 3.591 & 0.78116 & 9.116 & $47,501.3$ & $362,245.2$ & 2.775 \\
\hline 4.526 & 4.094 & 0.89058 & 8.251 & $33,806.2$ & $321,708.0$ & 1.070 \\
\hline 5.029 & 4.597 & 1.00000 & 8.313 & $33,806.2$ & $303,008.1$ & 0.000 \\
\hline
\end{tabular}

These parameters can now be modified to account for the differences between the UAE Phase V and Phase VI blades listed in Table 5.9. To do this, values of the inboard-most lineal density parameters listed in Table 6.4 are first lowered to account for the absence of the root-mounted camera in the UAE Phase VI blades (the Phase V camera mass is $13 \mathrm{~kg}$, equally distributed between the 0.508 and $1.006 \mathrm{~m}$ stations). Next, the mass distribution of the entire blade is adjusted to account for blade taper (the UAE Phase VI blades are larger near the root and smaller near the tip than the Phase V blades). This is done by multiplying the mass distribution by a linear function equal to unity at a span of $4.023 \mathrm{~m}$, the location where the chord length of the Phase VI blades equals the chord length of the Phase V blades (a position on the two blades where the lineal densities are most likely identical). The slope of this linear function is chosen to be $-0.021 / \mathrm{m}$ such that the c.g. of the blade is calculated to be $2.266 \mathrm{~m}$, the distance to the c.g. of the Phase VI blade from the center of rotation (the slope should be less than zero to ensure that the adjustment factor is smaller at the outboard stations and larger at the inboard stations). The magnitude of the resulting mass distribution is then scaled by a slight amount (0.966), so the calculated blade mass is equal to the actual measured blade mass $(60.2 \mathrm{~kg})$. The results of these steps are illustrated in Table 6.5. 


\section{Table 6.5: Distributed Mass Properties of the UAE Phase VI Blades}

\begin{tabular}{|l|r|r|r|r|}
\hline Distance & Blade & Properties of an Element Centered at $r$ \\
\cline { 3 - 5 } from Rotor & Lineal & Element & Element \\
Center & Density & Length & $\begin{array}{r}\text { Element } \\
\text { Mass }\end{array}$ & $\begin{array}{r}\text { Contribution } \\
\text { to c.g. } m\end{array}$ \\
\hline 0.402 & & & & \\
\hline 0.508 & 8.298 & 0.121 & 1.000 & 0.008 \\
\hline 0.749 & 22.109 & 0.249 & 5.505 & 0.068 \\
\hline 1.006 & 32.416 & 0.254 & 8.234 & 0.137 \\
\hline 1.257 & 28.750 & 0.252 & 7.231 & 0.151 \\
\hline 1.509 & 15.349 & 0.378 & 5.794 & 0.145 \\
\hline 2.012 & 12.115 & 0.503 & 6.094 & 0.203 \\
\hline 2.515 & 11.512 & 0.503 & 5.791 & 0.242 \\
\hline 3.018 & 10.611 & 0.503 & 5.332 & 0.267 \\
\hline 3.520 & 9.744 & 0.503 & 4.896 & 0.286 \\
\hline 4.023 & 8.806 & 0.503 & 4.430 & 0.296 \\
\hline 4.526 & 7.886 & 0.503 & 3.967 & 0.298 \\
\hline 5.029 & 7.861 & 0.252 & 1.977 & 0.165 \\
\hline \multicolumn{5}{|r|}{} \\
\hline TOTALS & & 4.521 & 60.250 & 2.267 \\
\hline
\end{tabular}

Now that a calibrated distributed lineal density of the Phase VI blades is acquired, the flapwise and edgewise stiffness distributions listed in Table 6.4 are adjusted to account for taper and tuned so the out-of-plane and in-plane natural frequencies predicted by program Modes match those given in Table 5.9 (approximately 7.282 and $8.981 \mathrm{~Hz}$ for the first natural modes, respectively). This is done by a procedure similar to that described in the previous paragraph. For the flapwise stiffness distribution, the slope of the linear function is chosen to be $-0.451 / \mathrm{m}$ and the magnitude scaling factor is chosen to be 1.50. For the edgewise stiffness distribution, the slope of the linear function is chosen to be $-0.051 / \mathrm{m}$ and the magnitude scaling factor is set to unity. Using these adjustment factors, the resulting natural frequencies are $7.28 \mathrm{~Hz}$ (first out-of-plane), $30.79 \mathrm{~Hz}$ (second out-of-plane), and $8.985 \mathrm{~Hz}$ (first in-plane), which are all very close to the values provided for the UAE Phase VI blade in Table 5.9. As was the case for the stiffness calibrations of the Phase V blades, the structural pretwist distribution is assumed to coincide with the aerodynamic twist distribution during this process since the actual distribution is unknown. Also, the end mass, $M_{T i p}$, is set equal to zero in this process because there are no tip brakes. Again, centrifugal stiffening effects are not accounted for in this process. A summary of the calibrated distributed structural properties of the UAE Phase VI blades is given in Table 6.6. 
Table 6.6: Calibrated Properties of the UAE Phase VI Blades

\begin{tabular}{|c|c|c|c|c|c|c|}
\hline $\begin{array}{l}\text { Distance } \\
\text { from Rotor } \\
\text { Center } \\
m\end{array}$ & $\begin{array}{l}\text { Distance } \\
\text { Along Blade } \\
\text { From Root }(r) \\
m\end{array}$ & $\begin{array}{l}\text { Radial } \\
\text { Fraction } \\
(r / 4.597 \mathrm{~m})\end{array}$ & $\begin{array}{r}\text { Blade } \\
\text { Lineal } \\
\text { Density } \\
\left(\mu_{B}\right) \mathrm{kg} / \mathrm{m}\end{array}$ & $\begin{array}{r}\text { Local } \\
\text { Flapwise } \\
\text { Stiffness } \\
(E I) \mathrm{Nm}^{2}\end{array}$ & $\begin{array}{r}\text { Local } \\
\text { Edgewise } \\
\text { Stiffness } \\
(E I) \mathrm{Nm}^{2}\end{array}$ & $\begin{array}{r}\text { Local } \\
\text { Structural } \\
\text { Twist }\left(\theta_{S}\right) \\
\text { deg }\end{array}$ \\
\hline 0.432 & 0.000 & 0.00000 & 0.000 & $511,398.4$ & $393,019.1$ & 0.000 \\
\hline 0.508 & 0.076 & 0.01653 & 8.298 & $2,506,356.0$ & $1,927,343.0$ & 0.000 \\
\hline 0.749 & 0.317 & 0.06896 & 22.109 & $3,483,529.2$ & $1,507,898.5$ & 0.000 \\
\hline 1.006 & 0.574 & 0.12486 & 32.416 & $1,810,745.8$ & $1,276,435.4$ & 6.593 \\
\hline 1.257 & 0.825 & 0.17946 & 28.750 & $1,144,892.0$ & $1,103,877.7$ & 20.040 \\
\hline 1.509 & 1.077 & 0.23428 & 15.349 & $535,063.2$ & $934,207.7$ & 14.315 \\
\hline 2.012 & 1.580 & 0.34370 & 12.115 & $307,733.2$ & $674,456.7$ & 7.454 \\
\hline 2.515 & 2.083 & 0.45312 & 11.512 & $224,056.5$ & $581,796.3$ & 3.702 \\
\hline 3.018 & 2.586 & 0.56254 & 10.611 & $165,678.0$ & $509,058.2$ & 1.621 \\
\hline 3.520 & 3.088 & 0.67174 & 9.744 & $111,920.2$ & $436,219.0$ & 0.421 \\
\hline 4.023 & 3.591 & 0.78116 & 8.806 & $71,251.9$ & $362,245.2$ & -0.381 \\
\hline 4.526 & 4.094 & 0.89058 & 7.886 & $39,205.7$ & $313,455.2$ & -1.111 \\
\hline 5.029 & 4.597 & 1.00000 & 7.861| & $27,702.2$ & $287,462.0$ & -1.815 \\
\hline
\end{tabular}

To obtain the proper mode shapes needed for the FAST_AD input file (local flapwise and edgewise blade mode shapes instead of out-of-plane and in-plane mode shapes) the distributed structural pretwist is zeroed out in the Modes input file and Modes is rerun. Centrifugal stiffening effects are finally accounted for in this process by specifying a typical rotor rotational speed of $72 \mathrm{rpm}$. A summary of the resulting mode shapes is given in Table 6.7.

Table 6.7: Mode Shapes of the UAE Phase VI Blades

\begin{tabular}{|c|c|r|r|r|}
\hline \multirow{2}{*}{$\begin{array}{c}\text { Shape } \\
\text { Function } \\
\text { Number }(b)\end{array}$} & $\begin{array}{c}\text { Shape } \\
\text { Function } \\
\left(\varphi_{b} \text { 's }\right)\end{array}$ & $\begin{array}{r}|c| \\
1^{\text {st }} \text { Mode Const. } \\
\text { Proportionality } \\
\text { Coef.s }\left(C_{l, b} \text { 's }\right)\end{array}$ & $\begin{array}{r}2^{\text {nd }} \text { Mode Const. } \\
\text { Proportionality } \\
\text { Coef.s }\left(C_{2, b} \text { 's }\right)\end{array}$ & $\begin{array}{r}1^{\text {st }} \text { Edgewise } \\
\text { Mode Const. } \\
\text { Proportionality } \\
\text { Coef.s }\left(C_{l, b} \text { 's }\right)\end{array}$ \\
\hline 2 & {$\left[r /\left(R-R_{H}\right)\right]^{2}$} & 0.448 & -2.067 & 1.023 \\
\hline 3 & {$\left[r /\left(R-R_{H}\right)\right]^{3}$} & -1.694 & 5.5 & -0.056 \\
\hline 4 & {$\left[r /\left(R-R_{H}\right)\right]^{4}$} & 8.139 & -27.516 & 1.351 \\
\hline 5 & {$\left[r /\left(R-R_{H}\right)\right]^{5}$} & -8.976 & 46.7 & -2.187 \\
\hline 6 & {$\left[r /\left(R-R_{H}\right)\right]^{6}$} & 3.083 & -21.616 & 0.869 \\
\hline
\end{tabular}

The modal damping ratio associated with the first natural frequency of the blades in both the flapwise and edgewise directions is assumed to be the same as the in-plane and out-of-plane damping ratios of the UAE Phase V blade, $0.9250 \%$ and $0.7650 \%$ respectively. The modal damping ratio associated with the second flapwise natural frequency of the blades is assumed to equal $1.345 \%$, typical for utility-scale turbines. Though not used for any outputs or postprocessed data presented in this report, the locations of the stations along the blade for local moment outputs are chosen to coincide with the pressure tap stations (see Table 5.10). The maximum number of constant length increments along the blades allowable for integration of 
aerodynamic and elastic forces is chosen (20). Consequently, each blade element is $0.22985 \mathrm{~m}$ in length $[(5.029 \mathrm{~m}-0.432 \mathrm{~m}) / 20]$.

Axial and tangential induction factors are included in all aerodynamics computations by the choice of "SWIRL" for the induction factor model parameter of the main FAST_AD input file. The default tolerance for induction factor convergence (0.005) is specified. Both the dynamic inflow and dynamic stall models are employed for all Blind Comparison simulations run. Following the practice of a well-respected Blind Comparison participant, the tower shadow halfwidth at the plane of the rotor is assumed to be $0.4 \mathrm{~m}$ and the velocity deficit at this same point is assumed to be 0.35 . Consequently, the dimensionless tower shadow width at the rotor plane (the tower shadow width at the rotor plane divided by the rotor radius) is specified as 0.15908 . The tower shadow models are switched off for the upwind rotor configurations.

The air density is defined to be the average air density listed in Table 5.11 for each Blind Comparison test scenario. Since the flow in the wind tunnel is virtually steady, homogenous, uniform, and fixed in direction (see section 5.2), constant hub height wind data are used instead of full-field wind data. The constant wind speed value is specified in a wind data file differently for each Blind Comparison test case simulated, by using the average wind speed values listed in Table 5.11. An example wind data input file is given in Appendix III for test case S0700000 of the Blind Comparison study of the UAE wind turbine. Wind data files for other Blind Comparison test scenarios are very similar.

In FAST_AD, each blade element can be modeled with its own unique airfoil table. Eight airfoil tables are used in each simulation (see Table 6.8 and Appendix IV). All airfoil data tables are the same ones used as, and are obtained from, a well-respected Blind Comparison participant who employed the AeroDyn aerodynamics subroutines during simulations. All the airfoil data table files whose names begin with "S809_CLN" represent dimensionless coefficient airfoil data for the S809 airfoil taken from the OSU wind tunnel at a Reynolds number of 750,000 and extrapolated to account for deep stall effects. The deep stall characteristics are found using a utility program titled FoilCheck, which employs the Viterna-Corrigan equations of post stall. An aspect ratio of 11 is used during the application of these equations. All but the last "S809_CLN" airfoil data table files are additionally modified to account for stall delay effects. The stall delay characteristics are found using a procedure similar to that outlined in Du and Selig (1998). 
Table 6.8: Airfoil Data Table File Descriptions

\begin{tabular}{|c|l|l|}
\hline $\begin{array}{c}\text { Table } \\
\text { Identifier }\end{array}$ & Filename & File Description \\
\hline 1 & cylinder.dat & $\begin{array}{l}\text { Dimensionless coefficient airfoil data for a } \\
\text { smooth circular cylinder }\end{array}$ \\
\hline 2 & S809_CLN_129.dat & $\begin{array}{l}\text { Dimensionless coefficient airfoil data for the } \\
\text { S809 airfoil at a span of 12.9\% }\end{array}$ \\
\hline 3 & S809_CLN_189.dat & $\begin{array}{l}\text { Dimensionless coefficient airfoil data for the } \\
\text { S809 airfoil at a span of 18.9\% }\end{array}$ \\
\hline 4 & S809_CLN_242.dat & $\begin{array}{l}\text { Dimensionless coefficient airfoil data for the } \\
\text { S809 airfoil at a span of 24.2\% }\end{array}$ \\
\hline 5 & S809_CLN_298.dat & $\begin{array}{l}\text { Dimensionless coefficient airfoil data for the } \\
\text { S809 airfoil at a span of 29.8\% }\end{array}$ \\
\hline 6 & S809_CLN_354.dat & $\begin{array}{l}\text { Dimensionless coefficient airfoil data for the } \\
\text { S809 airfoil at a span of 35.4\% }\end{array}$ \\
\hline 7 & S809_CLN_410.dat & $\begin{array}{l}\text { Dimensionless coefficient airfoil data for the } \\
\text { S809 airfoil at a span of 41.0\% }\end{array}$ \\
\hline 8 & S809_CLN_Outboard.dat & $\begin{array}{l}\text { Dimensionless coefficient airfoil data for the } \\
\text { S809 airfoil at all other outboard span stations }\end{array}$ \\
\hline
\end{tabular}

Airfoil data tables are correlated to each blade element by prescribing an airfoil table identifier at the node between each element. Since 20 elements are chosen for integrating aerodynamic and elastic forces, there are 21 such nodes. The first is located at a distance along the blade axis from the center of rotation equal to that of the hub radius (taken here to be $0.432 \mathrm{~m}$ ) and the last node is located at the blade tip. Since FAST_AD requires each blade element to have the same length, he nodal locations cannot be aligned with the exact span station for which each airfoil data table is derived. Therefore, the airfoil data tables are matched to the most well-suited node. Aerodynamic properties associated with each node are listed in Table 6.9. 
Table 6.9: Nodal Aerodynamic Properties

\begin{tabular}{|c|c|c|c|c|c|c|c|c|}
\hline \multirow[b]{2}{*}{$\begin{array}{c}\text { Node } \\
\text { Number }\end{array}$} & \multicolumn{4}{|c|}{ Node Location } & \multirow{2}{*}{\begin{tabular}{|c|} 
Prescribed \\
Airfoil \\
Data \\
Table \\
Identifier
\end{tabular}} & \multirow[b]{2}{*}{$\begin{array}{r}\text { Local } \\
\text { Chord } \\
\text { Length }(c) \\
m\end{array}$} & \multirow[b]{2}{*}{$\begin{array}{r}\text { Local } \\
\text { Twist }\left(\theta_{T}\right) \\
d e g\end{array}$} & \multirow[b]{2}{*}{$\begin{array}{r}\text { Local } \\
\text { Thickness } \\
m\end{array}$} \\
\hline & $\begin{array}{l}\text { Distance } \\
\text { from Rotor } \\
\text { Center } \\
m\end{array}$ & \begin{tabular}{|l} 
Spanwise \\
Fraction \\
$(/ 5.029 \mathrm{~m})$
\end{tabular} & $\begin{array}{l}\text { Distance } \\
\text { Along Blade } \\
\text { From Root }(r) \\
m\end{array}$ & $\begin{array}{l}\text { Radial } \\
\text { Fraction } \\
(r / 4.597 \mathrm{~m})\end{array}$ & & & & \\
\hline 1 & 0.432 & 0.086 & 0.000 & 0.000 & 1 & 0.218 & 0.00 & 1.000 \\
\hline 2 & 0.662 & 0.132 & 0.230 & 0.050 & 2 & 0.209 & 0.00 & 1.000 \\
\hline 3 & 0.892 & 0.177 & 0.460 & 0.100 & 3 & 0.283 & 3.66 & 0.707 \\
\hline 4 & 1.122 & 0.223 & 0.690 & 0.150 & 4 & 0.526 & 12.78 & 0.351 \\
\hline 5 & 1.351 & 0.269 & 0.919 & 0.200 & 4 & 0.727 & 17.89 & 0.209 \\
\hline 6 & 1.581 & 0.314 & 1.149 & 0.250 & 5 & 0.704 & 13.33 & 0.209 \\
\hline 7 & 1.811 & 0.360 & 1.379 & 0.300 & 6 & 0.680 & 10.19 & 0.209 \\
\hline 8 & 2.041 & 0.406 & 1.609 & 0.350 & 7 & 0.657 & 7.24 & 0.209 \\
\hline 9 & 2.271 & 0.452 & 1.839 & 0.400 & 8 & 0.634 & 5.52 & 0.209 \\
\hline 10 & 2.501 & 0.497 & 2.069 & 0.450 & 8 & 0.611 & 3.81 & 0.209 \\
\hline 11 & 2.731 & 0.543 & 2.299 & 0.500 & 8 & 0.588 & 2.81 & 0.209 \\
\hline 12 & 2.960 & 0.589 & 2.528 & 0.550 & 8 & 0.565 & 1.86 & 0.209 \\
\hline 13 & 3.190 & 0.634 & 2.758 & 0.600 & 8 & 0.542 & 1.21 & 0.209 \\
\hline 14 & 3.420 & 0.680 & 2.988 & 0.650 & 8 & 0.518 & 0.66 & 0.209 \\
\hline 15 & 3.650 & 0.726 & 3.218 & 0.700 & 8 & 0.495 & 0.21 & 0.209 \\
\hline 16 & 3.880 & 0.771 & 3.448 & 0.750 & 8 & 0.472 & -0.15 & 0.209 \\
\hline 17 & 4.110 & 0.817 & 3.678 & 0.800 & 8 & 0.448 & -0.51 & 0.209 \\
\hline 18 & 4.339 & 0.863 & 3.907 & 0.850 & 8 & 0.425 & -0.84 & 0.209 \\
\hline 19 & 4.569 & 0.909 & 4.137 & 0.900 & 8 & 0.402 & -1.17 & 0.209 \\
\hline 20 & 4.799 & 0.954 & 4.367 & 0.950 & 8 & 0.378 & -1.49 & 0.209 \\
\hline 21 & 5.029 & 1.000 & 4.597 & 1.000 & 8 & 0.355 & -1.82 & 0.209 \\
\hline
\end{tabular}

One of the final sets of parameters defined in the FAST_AD input file is a list of desired output channels. Of the numerous output channels available, those requested include instantaneous simulation clock time, nacelle yaw angle, wind direction, wind speed, Blade 1 azimuth angle position, Blade 1 out-of-plane tip deflection, Blade 1 pitch angle, rotor rotational speed, Blade 1 out-of-plane and in-plane bending moments, low-speed shaft torque, and nacelle yaw moment. Additionally, detailed aerodynamic data, including instantaneous angle of attack, lift and drag coefficients, axial and tangential induction factors, differential thrust, and differential torque force $^{30}$, are requested at several nodes between the blade elements. This is achieved by setting the "PRINT" flag at each node. Since the nodal locations cannot be aligned with the exact span stations where the pressure tap groupings are positioned (see discussion in the previous paragraph), the detailed instantaneous aerodynamic data are requested at the two nodes surrounding each pressure tap grouping span station unless the station is significantly closer to one of the two adjoining nodes, in which case, only the closest node is chosen. Comparing the pressure tap grouping span stations given in Table 5.10 with the node locations given in Table

\footnotetext{
${ }^{30}$ The differential thrust and differential torque force output channels are labeled as ForcN and ForcT respectively and are identified as differential normal and tangential forces acting on the blade element in the YawDyn User's Guide by Hansen and Laino (1998). This identification is misleading since these forces are not perpendicular and parallel to the local chord line, which is the UAE's measurement convention. Instead, these forces are defined to be normal and tangent to the plane of rotation, respectively, for blades with no coning, which are more commonly identified as thrust and torque forces.
} 
6.9, the "PRINT" flag is seen to be most properly designated at nodes 5, 6, 9, 10, 13, 16, 17, and 20. The $30 \%$ span pressure tap grouping is located $68.431 \%$ of the way between nodes 5 and 6 , the $46.67 \%$ span pressure tap grouping is located $33.093 \%$ of the way between nodes 9 and 10 , and the $80 \%$ span pressure tap grouping is located $62.407 \%$ of the way between nodes 16 and 17 . The $63.33 \%$ span pressure tap grouping is aligned very closely with node 13 and the $95 \%$ span pressure tap grouping is aligned very closely with node 20 .

For each simulation run, these output channels yield time-series data on the loads and responses of the selected members of the wind turbine modeled. That is, at each time increment between the simulation time of 20 and $30 \mathrm{~s}$, an instantaneous value of each output channel is listed. The time-series data associated with the aerodynamic parameters of the blade nodes are given in a separate file than the time-series data for the other output channels selected. After each simulation is run, these two output data files are concatenated into one file using an MS Excel spreadsheet. All cells are formatted to retain 6 decimal places to minimize any round-off errors. Next, the post-processing code Crunch is employed to use these data and develop the output channels and associated measurement conventions requested for the Blind Comparison exercise and to azimuth average the resulting time series data into $1^{\circ}$ azimuth angle bins. The resulting data file, which contains 360 rows of azimuth averaged time series data of the 45 output channels requested for the Blind Comparison exercise, is finally imported into a reporting spreadsheet for ease in developing plots of the results. Unfortunately, the version of Crunch employed (2.60) does not allow for selection of bin centers, so the azimuth averaged values are offset by $0.5^{\circ}$ from Blind Comparison output convention.

The equations employed in Crunch to develop the output channels and associated measurement conventions requested for the Blind Comparison exercise are relatively simple. They are described qualitatively and quantitatively here, and are given in detail in the example Crunch input file of Appendix V for the test case S0700000. The Crunch input files for the other test cases are the same as this input file except for the last line: the name of the input file from which to read the time series data.

The yaw error, $Y A W$, is found by subtracting the wind direction (which is always zero) from the nacelle yaw position. The Blade 3 flap angle of the UAE wind turbine, B3FLAP, is found by summing the precone angle and the inverse tangent of the ratio of the Blade 1 out-of-plane tip deflection (of the FAST_AD turbine) and the rotor radius:

$$
B 3 F L A P=\beta_{1}+\operatorname{atan}\left(\frac{\text { Blade1Out }- \text { Of }- \text { Plane Tip Displacement }}{R}\right)
$$

To orient the moments with respect to the tip chord line properly, the Blade 3 root flap and root edge bending moments of the UAE wind turbine, B3RFP and B3REP respectively, are found by applying the following vectorial relationships between the in-plane and out-of-plane bending moments outputted by FAST_AD: 


$$
\begin{aligned}
& B 3 R F P=(\text { Out }- \text { Of }- \text { Plane Moment }) \times \cos \left(3.0^{\circ}\right)+ \\
&(\text { In }- \text { Plane Moment }) \times \sin \left(3.0^{\circ}\right) \\
& \text { and } \\
& B 3 R E P=- \\
&(\text { Out }- \text { Of }- \text { Plane Moment }) \times \sin \left(3.0^{\circ}\right)+ \\
&(\text { In }- \text { Plane Moment }) \times \cos \left(3.0^{\circ}\right)
\end{aligned}
$$

To ensure the proper sign convention, the nacelle yaw moment of the UAE wind turbine, $N A Y M$, is found by multiplying the nacelle yaw moment output by FAST_AD by -1 . In addition, all the torque and moment outputs provided by FAST_AD are multiplied by 1,000 , since FAST_AD outputs moments in $\mathrm{kNm}$ and the unit convention for the Blind Comparison exercise is $\mathrm{Nm}$.

For the cases in which the nodes do not line up with the pressure tap grouping span locations, linear interpolations are made between the two adjoining nodes to determine the aerodynamic properties at the correct span locations. This is done to find the local dimensionless normal and tangential force coefficients, local normal and tangential forces per unit span, and local dynamic pressures at each of the five pressure tap grouping span stations. Since FAST_AD cannot compute pitching moments or dimensionless pitching moment coefficients, all these Blind Comparison Output channels are zeroed out.

Dimensionless normal and tangential force coefficients are derived from the dimensionless lift and drag coefficients provided at each node via forms of Eqs. (2.43) and (2.44) (the forces in these equations are replaced with force coefficients). Normal and tangential forces per unit span are derived from the differential thrust and differential torque forces ( $d Q$ divided by $r$ ) through the inverses of Eqs. (2.57) and (2.58) divided by the blade element length, $d r$ :

$$
\begin{gathered}
\text { Normal Force } / \text { Unit Span }=\frac{1}{d r}\left[d T \cos \left(\theta_{P T}\right)+\left(\frac{d Q}{r}\right) \sin \left(\theta_{P T}\right)\right] \\
\text { and } \\
\text { Tangential Force /Unit Span }=\frac{1}{d r}\left[-d T \sin \left(\theta_{P T}\right)+\left(\frac{d Q}{r}\right) \cos \left(\theta_{P T}\right)\right]
\end{gathered}
$$

The local dynamic pressure at each node is backed out of the previous results by dividing the force per unit span by its associated dimensionless coefficient and the local chord length. Since this can be done with either the normal and tangential forces and associated dimensionless force coefficients, the average of the two is taken.

\subsection{Blind Comparison Results and Discussion}

After the FAST_AD input files(s) are fully developed, simulations are easy to run and output data are obtained in minutes. Careful and meaningful examination of these data is more difficult. A comparison and discussion of some load predictions made by FAST_AD and other Blind 
Comparison participants and data obtained experimentally in the NASA-Ames wind tunnel are given here. Just as the Blind Comparison exercise opted to target specific aerodynamic phenomena, so too, this section focuses on particular aerodynamic events. Since a prudent study of the more complicated downwind and nonzero yaw error cases cannot be undertaken before discrepancies in the simplest test cases are first understood, significantly more attention is paid to the upwind rotor configuration, zero yaw error cases. Moreover, concentration is given to comparing and discussing aerodynamic properties, including dimensionless force coefficients, local dynamic pressures, and the like. To facilitate validation of FAST_AD, trends in discrepancies between the modelers' predictions are noted and discrepancies between the modelers' predictions and those measured during the NASA-Ames tests are highlighted. Potential sources of the discrepancies and their effects on the design of wind turbine systems are noted. Probable causes of discrepancies are discussed further in the next chapter.

Average dimensionless normal force coefficients at each pressure tap grouping span station are plotted against wind speed on the abscissa in Figs. 6.2-6.6. Corresponding local dynamic pressures are given in Figs. 6.7-6.11. All these data represent measurements and code predictions for the upwind rotor configuration, zero yaw error test cases at wind speeds of 7, 10, $13,15,20$ and $25 \mathrm{~m} / \mathrm{s}$ ( $\mathrm{S}^{* *} 00000$-series Blind Comparison test cases). The curves labeled "NREL" represent the data measured during the NASA-Ames wind tunnel tests. The light curves labeled "Others" represent data predicted by other Blind Comparison participants who employed the AeroDyn aerodynamics subroutines.

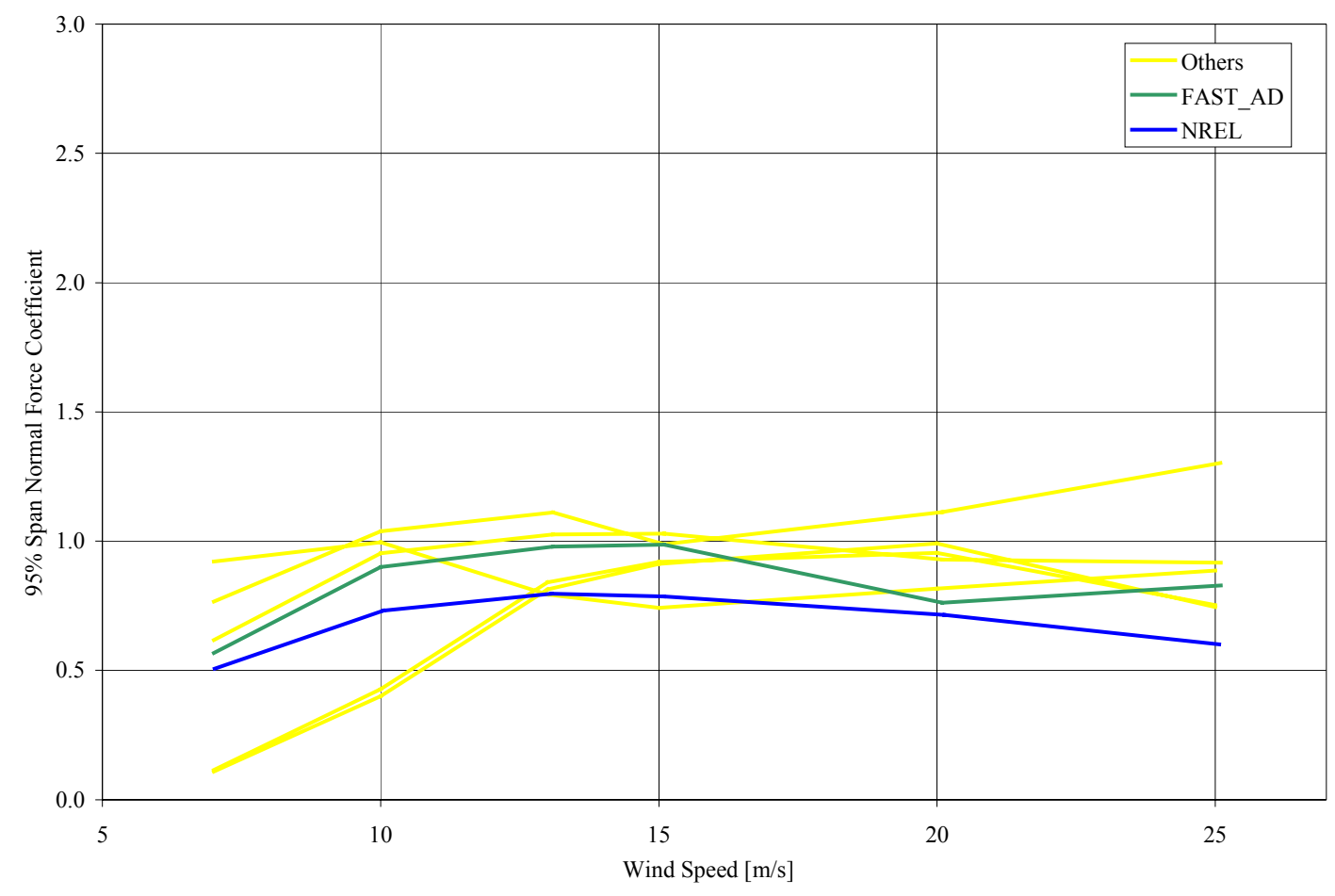

Figure 6.2: 95\% span normal force coefficient, upwind, zero yaw 


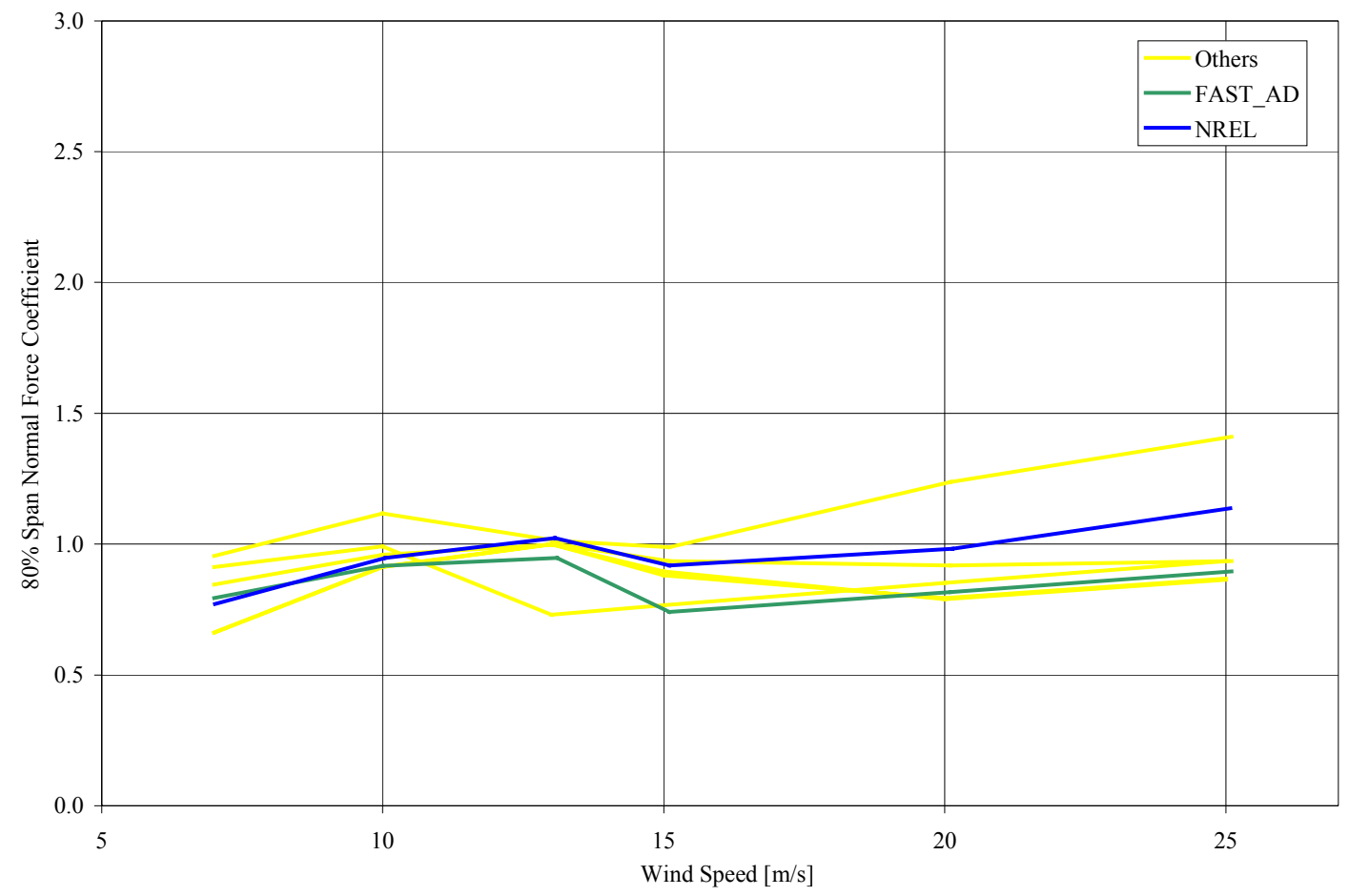

Figure 6.3: $80 \%$ span normal force coefficient, upwind, zero yaw

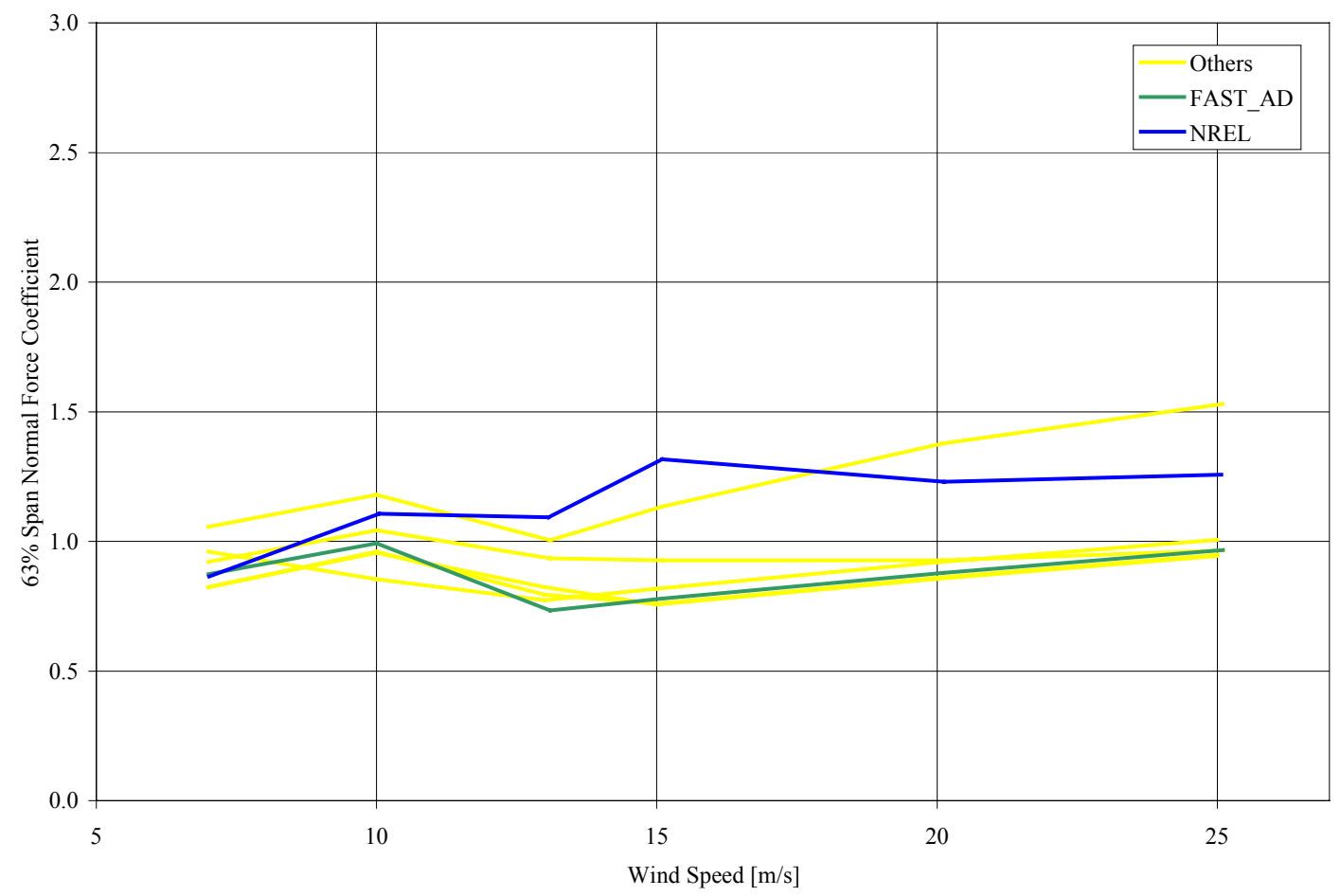

Figure 6.4: 63\% span normal force coefficient, upwind, zero yaw 


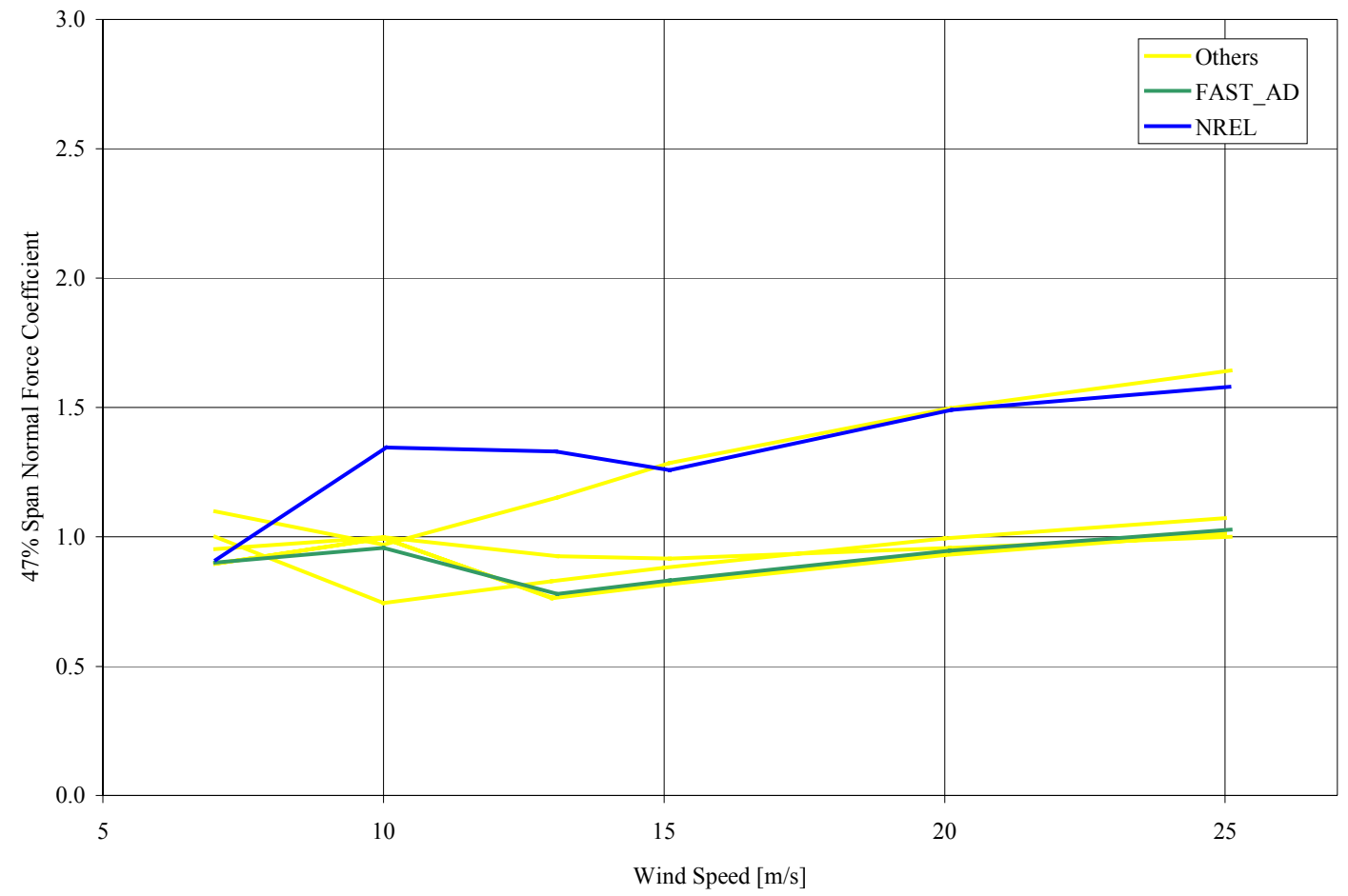

Figure 6.5: $47 \%$ span normal force coefficient, upwind, zero yaw

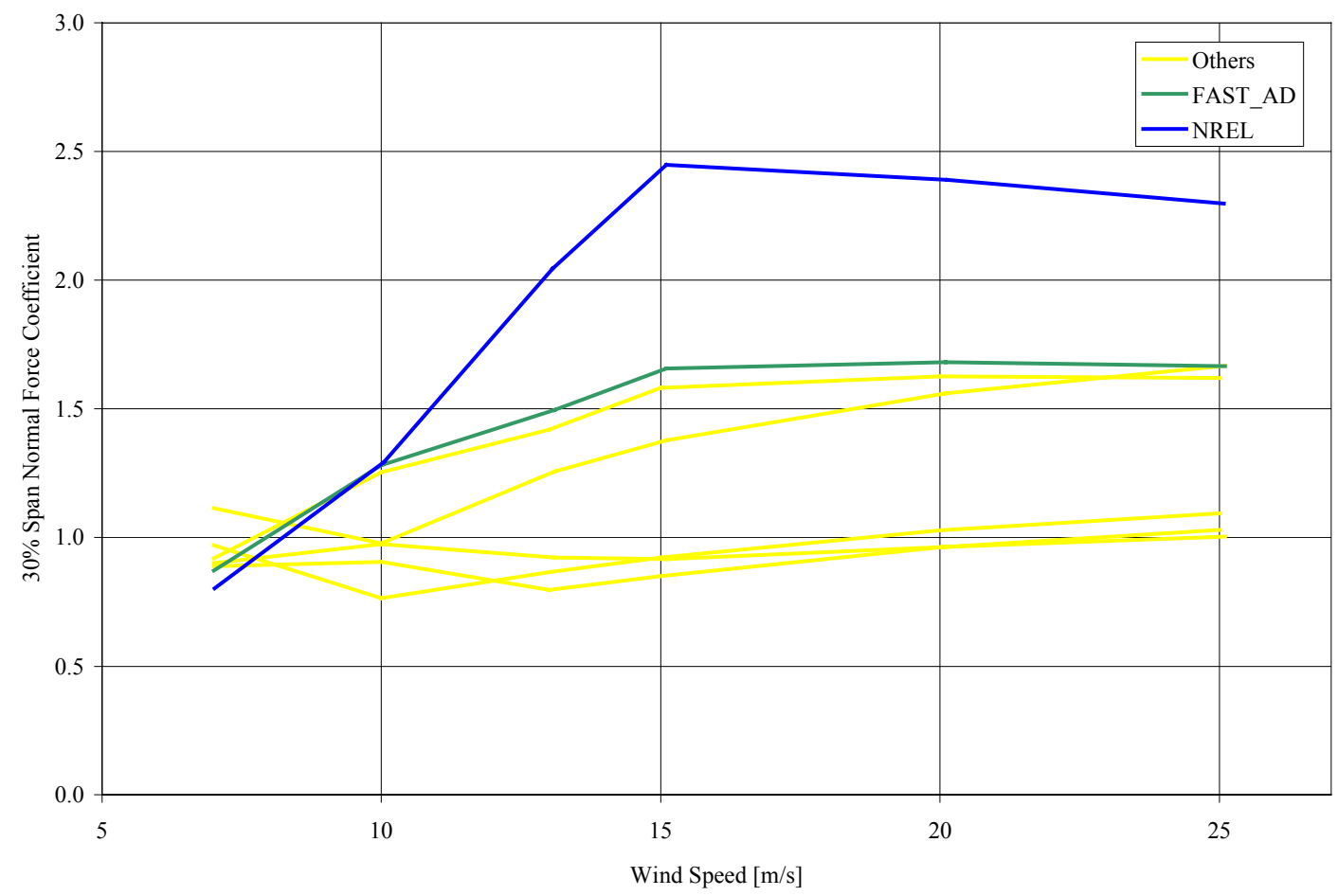

Figure 6.6: $30 \%$ span normal force coefficient, upwind, zero yaw 


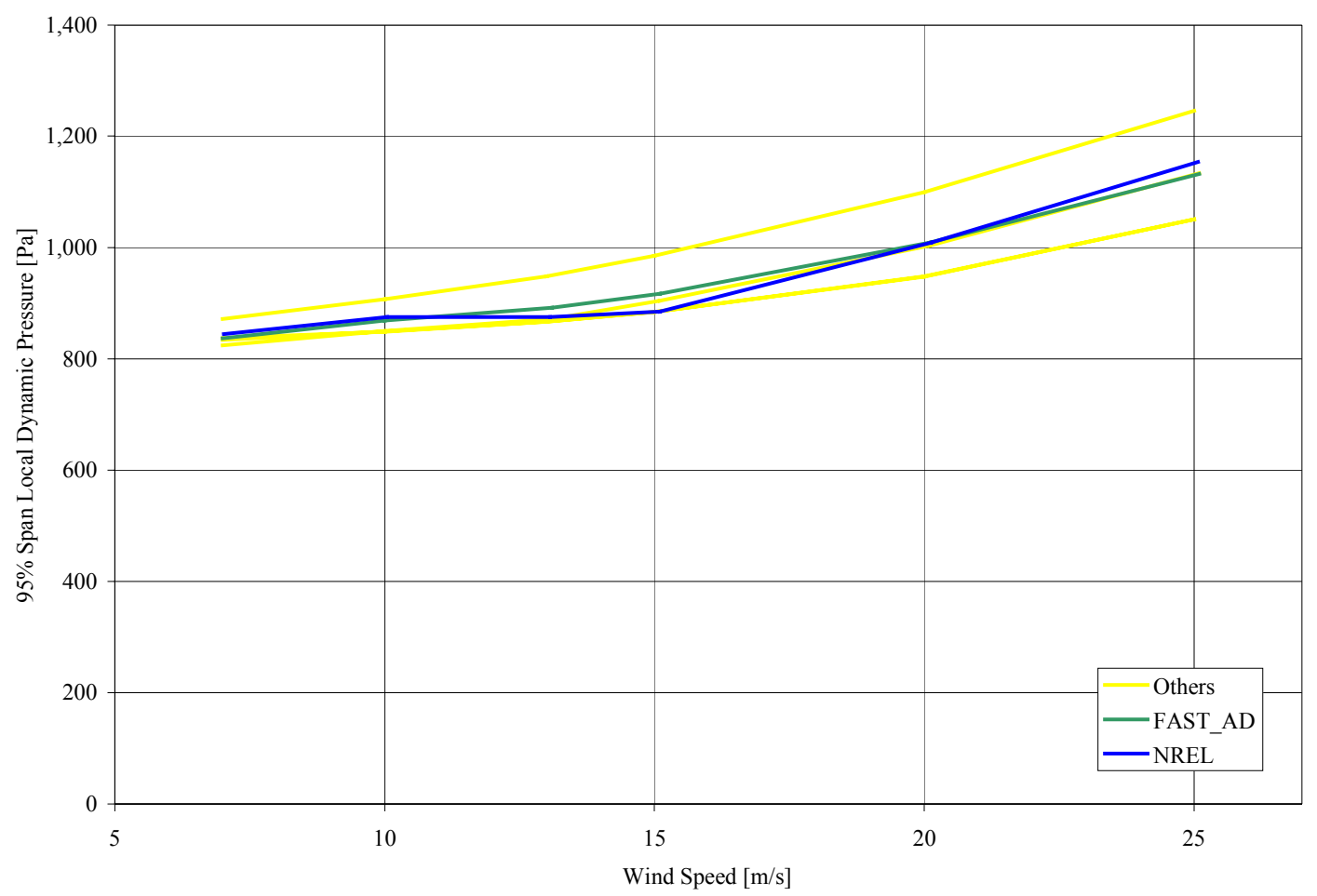

Figure 6.7: 95\% span local dynamic pressure, upwind, zero yaw

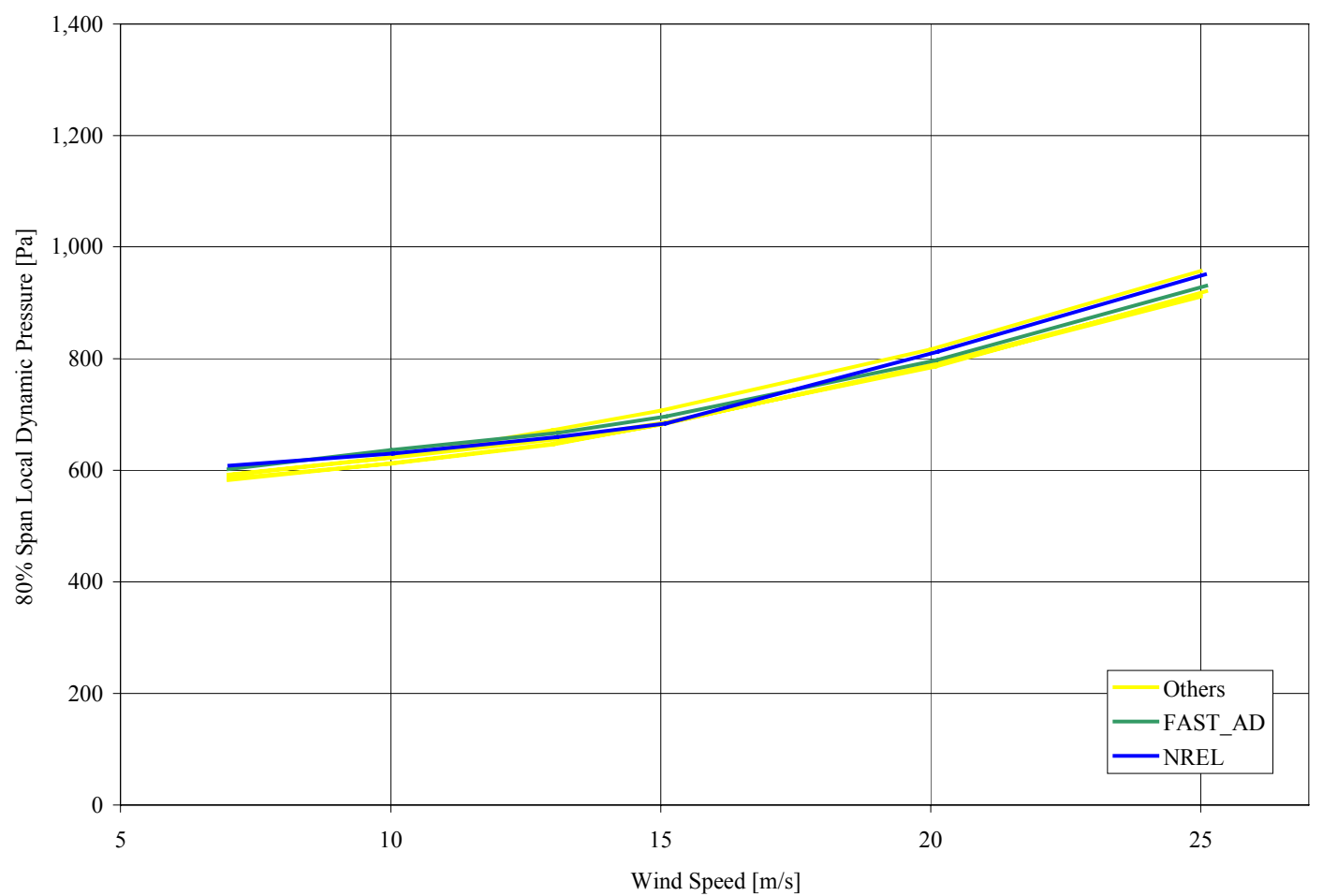

Figure 6.8: $80 \%$ span local dynamic pressure, upwind, zero yaw 


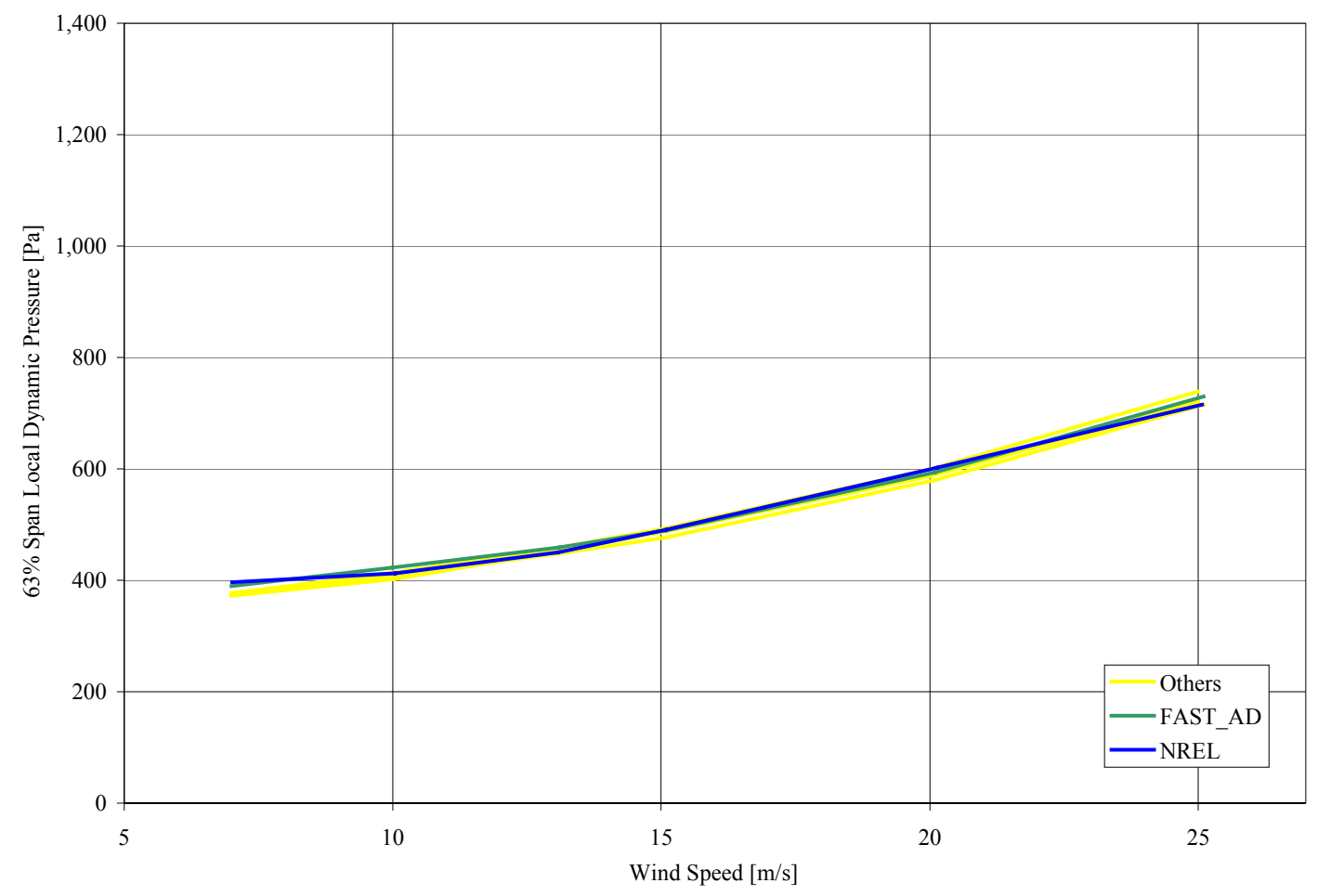

Figure 6.9: 63\% span local dynamic pressure, upwind, zero yaw

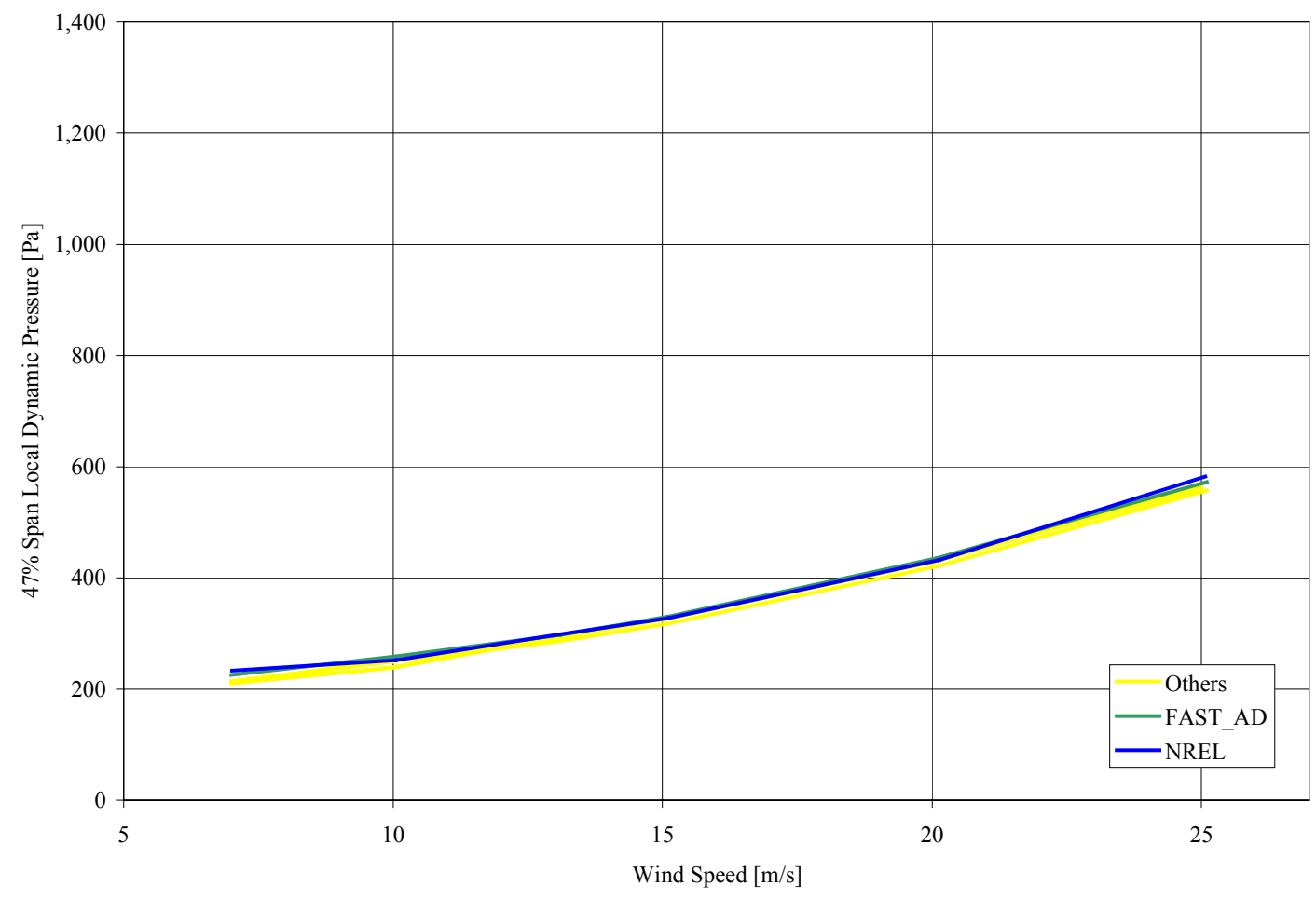

Figure 6.10: 47\% span local dynamic pressure, upwind, zero yaw 


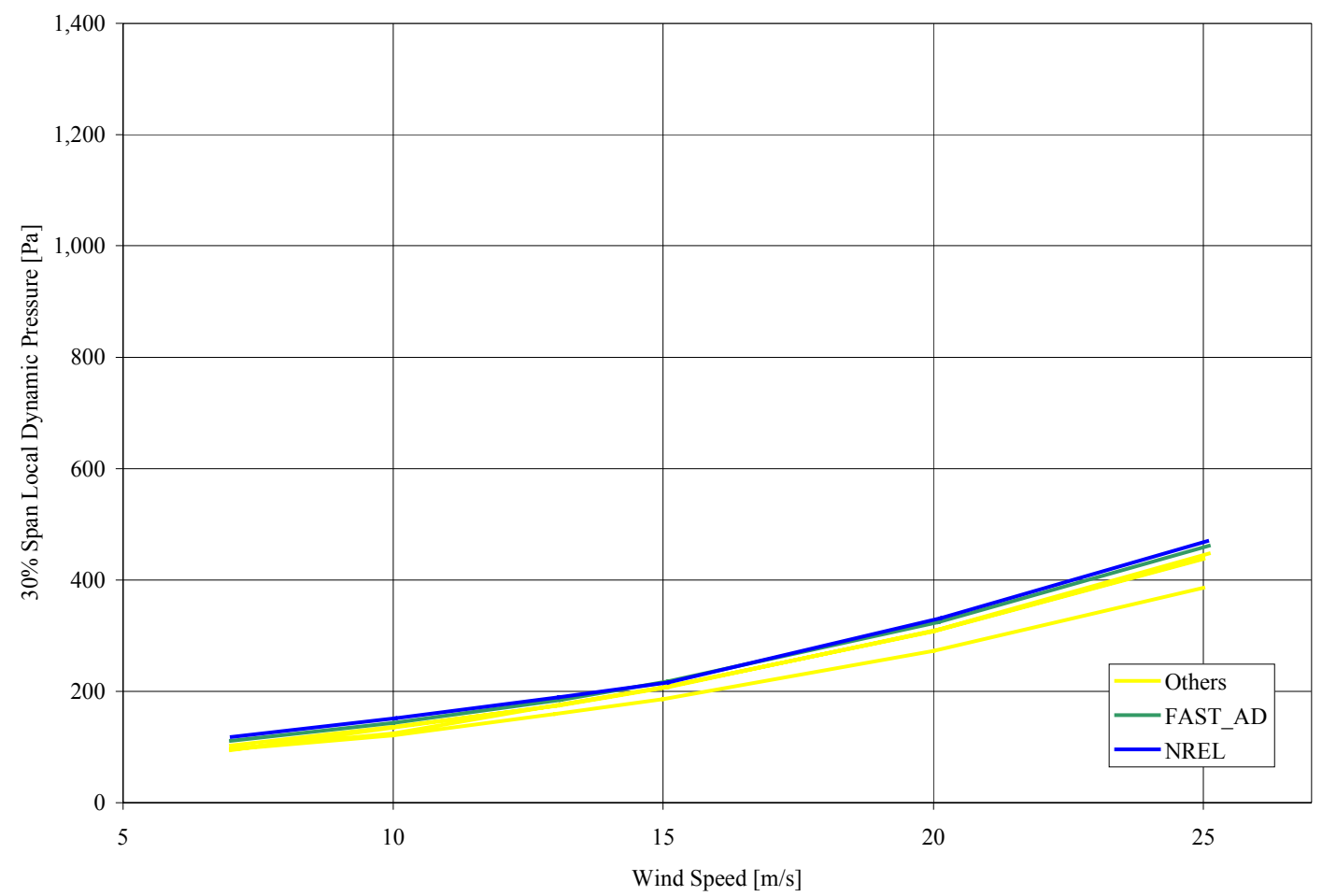

Figure $6.11: 30 \%$ span local dynamic pressure, upwind, zero yaw

All modelers tend to overpredict dimensionless normal aerodynamic force coefficients at the outermost span location (95\% span). This reveals that Prandtl's tip loss factor (see section 2.5) may not properly account for the reduction in aerodynamic forces near the tip. Proper characterization of aerodynamic forces along the outboard portions of a blade is critical to successfully design wind turbines, since most rotor torque is generated from these blade sections when operating at or below the rated wind speed of the turbine.

In contrast, all modelers tend to underpredict dimensionless normal force coefficients at the innermost span location ( $30 \%$ span), suggesting that more work needs to be done to improve stall delay models and models that account for the three-dimensional effects at the blade root. Flow around this part of a wind turbine blade is very complex as a result of flow disturbances around the hub (and around the nacelle for downwind rotor configurations). Consequently, refining these models may be a difficult or impossible. Proper characterization of aerodynamic forces along the inboard parts of a blade is especially critical when analyzing wind turbines that operate at high wind speeds (above rated speed) since the outboard parts of the blade are in deep stall and most rotor torque is generated from the innermost blade sections because of stall delay.

Most modelers also underpredict dimensionless normal force coefficients at the midboard span locations. The cause of these discrepancies is most likely a combination of the discrepancy causes previously discussed.

All modelers predicted local dynamic pressures accurately at all span locations and wind speeds for the upwind, zero yaw error test cases. As a result, the local normal aerodynamic force curves (not shown) follow similar trends to the curves of the dimensionless normal force coefficients. 
FAST_AD predicts local dynamic pressures more accurately than the other codes in almost every instance.

From a global standpoint, data scatter between load predictions tends to increase with wind speed. This indicates that post-stall models, such as the commonly used Viterna-Corrigan model (see section 2.4), are deficient. Such models will have to be refined if they are to be useful in the development of stall-regulated wind turbines. A significant fraction of a stall-controlled wind turbine blade operates in the post-stall regime throughout all but the smallest wind-inflows.

Another probable cause of increasing data scatter with increasing wind speed is that the drag coefficient is neglected when iterating to find the operational state of the wind turbine in the AeroDyn aerodynamics subroutines. Since the UAE research wind turbine is a stall-regulated machine and there is significant drag in the post-stall regime, drag is not negligible, and AeroDyn is limited in its ability to properly model these type of operational states (see section 4.2). AeroDyn Code developers should consider revising the aerodynamics models in future versions of the code by having them include the effects of drag when iterating to find the operational state. That is, the code should be modified to use a form of Eq. (2.70) instead of a form of Eq. (2.73). Aerodynamic load predictions in the post stall regime will undoubtedly be improved by this action.

To examine the collective effect of the aerodynamic force discrepancies, plots of low-speed shaft torque and root flap bending moments for the same six cases are given in Figs. 6.12 and 6.13:

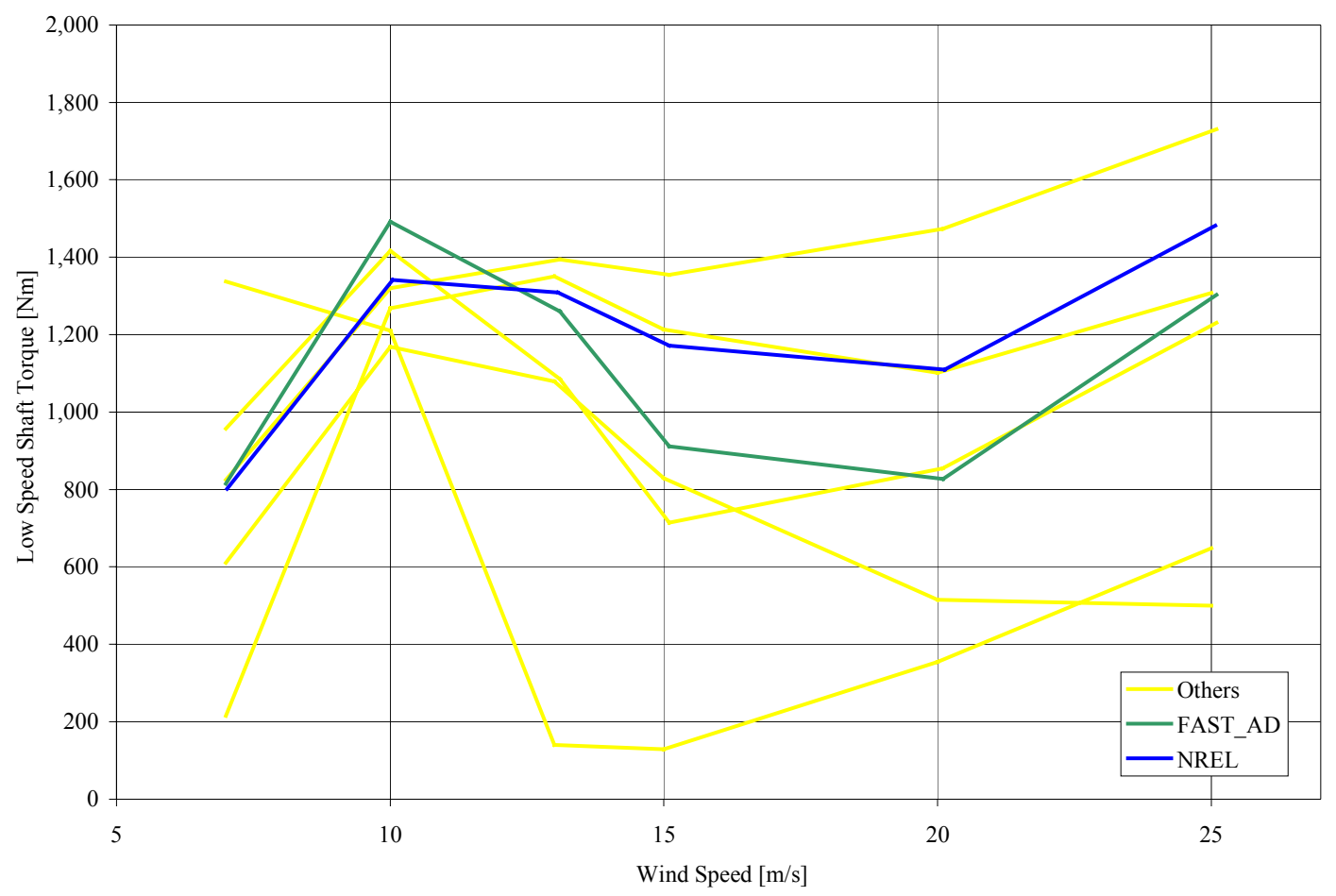

Figure 6.12: Low-speed shaft torque, upwind, zero yaw 


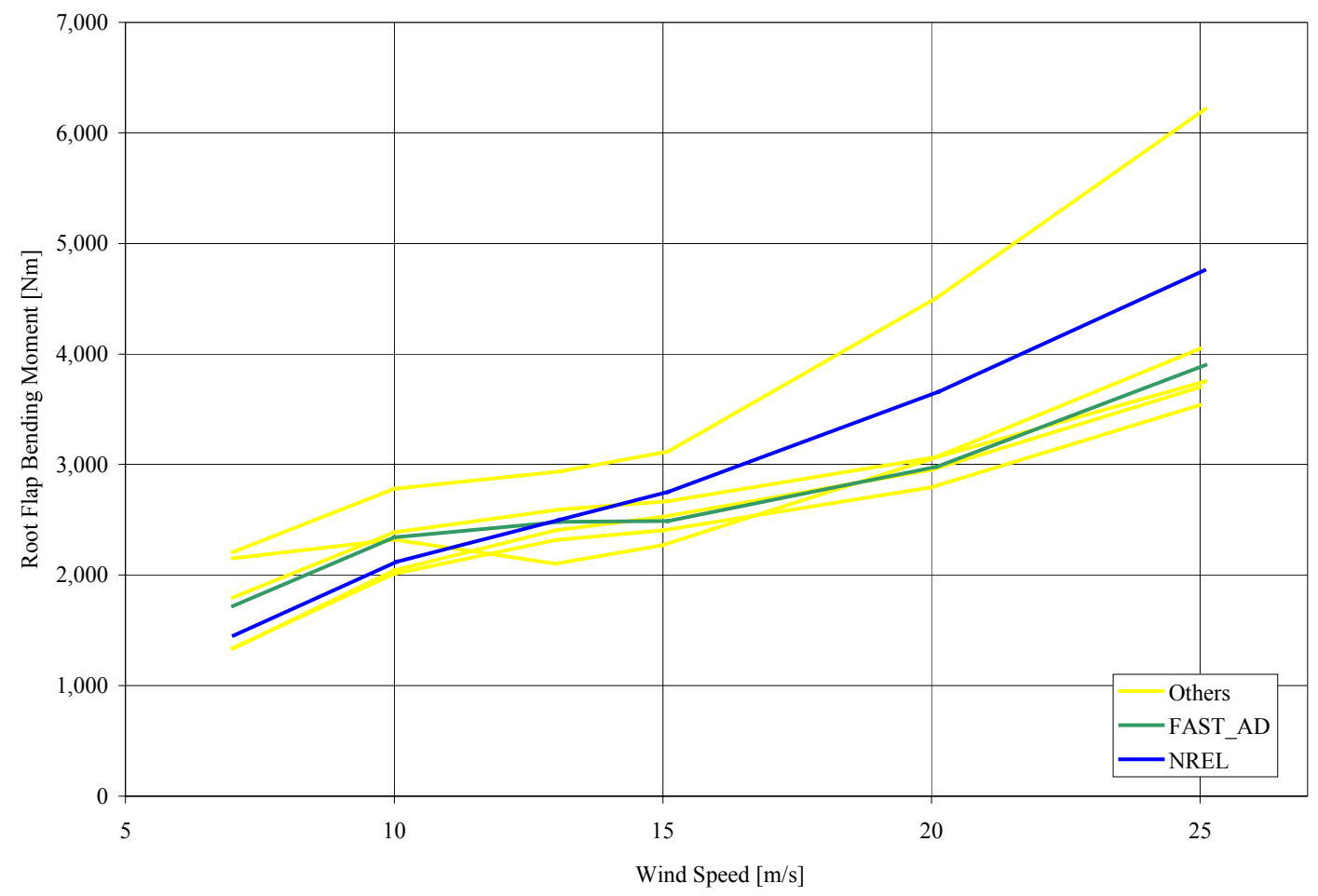

Figure 6.13: Root flap bending moment, upwind, zero yaw

Modeling tool predictions of the low-speed shaft torque are $10 \%-170 \%$ of their experimentally measured counterparts. Predictions of the root flap bending moment are $70 \%-160 \%$ of the measured values. In many instances, overpredictions of local aerodynamic forces on some parts of the blade and underpredictions of local aerodynamic forces on others most likely mitigated the discrepancies in these torque and moment predictions. However, from a structural standpoint, optimal blades cannot be designed properly if the assumed load distribution is erroneous.

Like the aerodynamic force predictions, torque and moment prediction discrepancies tend to increase with wind speed; the causes of the discrepancies in the former predictions are most likely the causes for the discrepancies in the latter. Nevertheless, as demonstrated for the FAST_AD model in Chapter 7, even complete eliminating aerodynamic force discrepancies does not eliminate discrepancies in the torque and moment predictions.

Azimuth averaged dimensionless normal force coefficients at 47\% span are plotted against the azimuth angle on the abscissa for the downwind rotor configuration, zero yaw error, 7 and 17 $\mathrm{m} / \mathrm{s}$ test cases ( $\mathrm{E}^{* *} 00000 \mathrm{~A}$-series) in Figs. 6.14 and 6.15. These plots highlight deficiencies in the tower shadow model employed in the AeroDyn aerodynamics subroutines. At a wind speed of $7 \mathrm{~m} / \mathrm{s}$, all modelers apparently underpredicted the tower shadow width. In this case, some modelers apparently overpredicted and others underpredicted the velocity deficit. At a wind speed of $17 \mathrm{~m} / \mathrm{s}$, all modelers properly estimated the tower shadow width, but overpredicted all the velocity deficits. In either case, more work is needed to improve tower shadow models and the methods of predicting tower shadow features. This is crucial for the successful design of downwind turbines. 


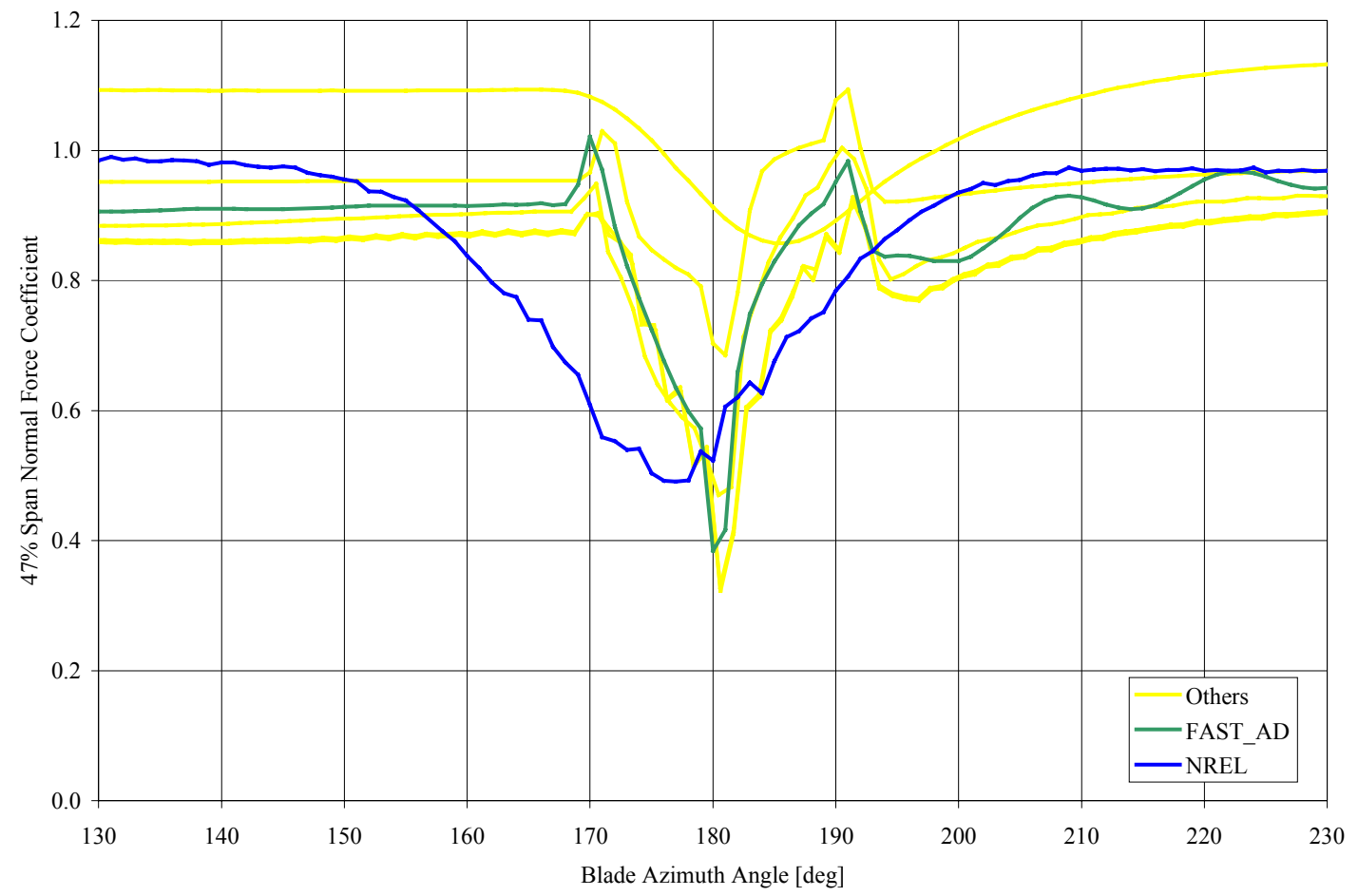

Figure 6.14: $47 \%$ span normal force coefficient, $7 \mathrm{~m} / \mathrm{s}$, downwind, zero yaw

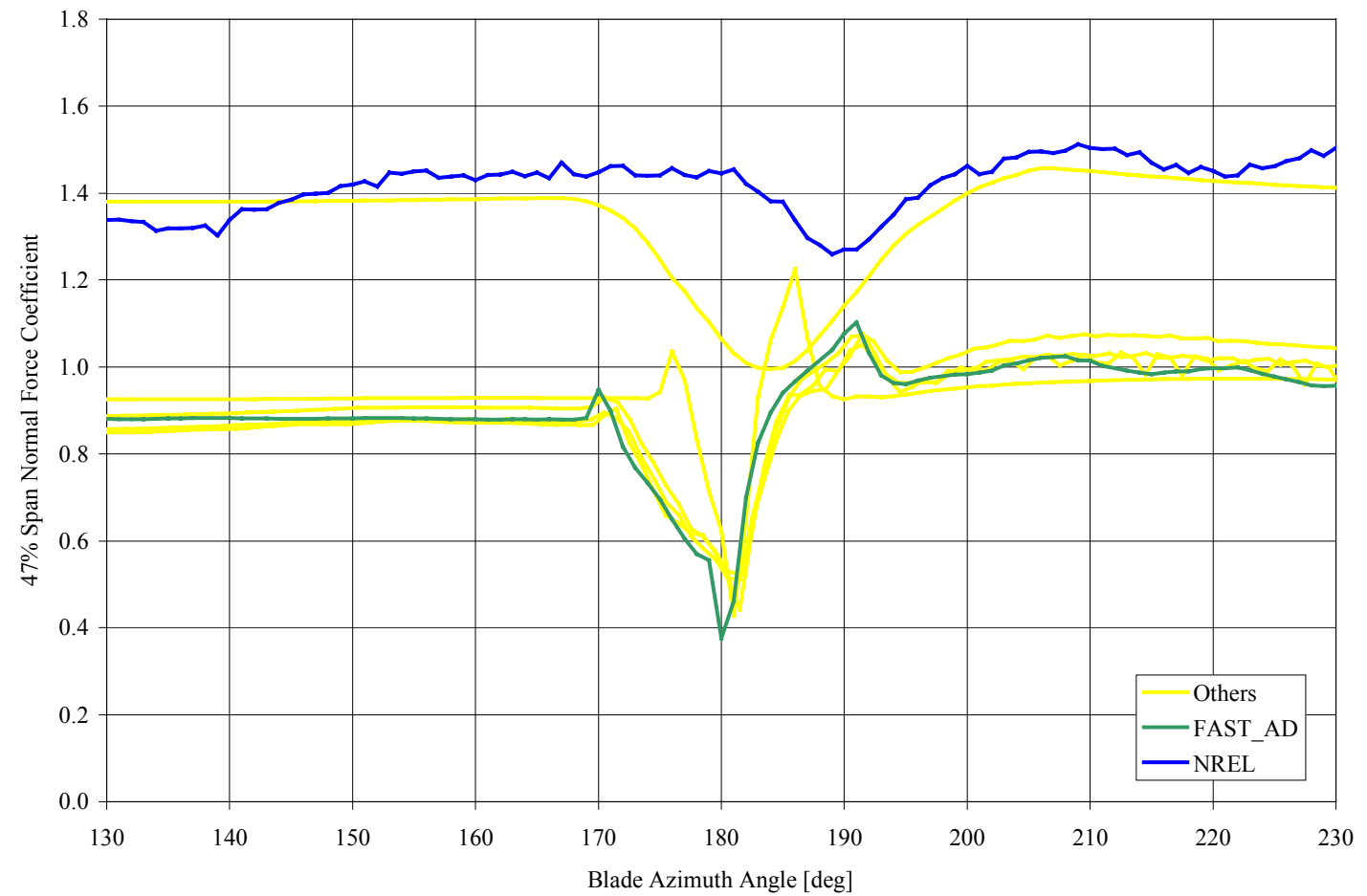

Figure 6.15: $47 \%$ span normal force coefficient, $17 \mathrm{~m} / \mathrm{s}$, downwind, zero yaw

Azimuth averaged dimensionless normal force coefficients and local dynamic pressures for several span stations are plotted against the azimuth angle on the abscissa for the upwind rotor 
configuration, $30^{\circ}$ yaw error for the $15 \mathrm{~m} / \mathrm{s}$ test case (S1500300) in Figs. 6.16-6.21. Though the causes of discrepancies in the aerodynamic forces all apply in this test case, these plots also highlight deficiencies in the Beddoes-Leishman dynamic stall model. In general, predictions of the dynamic pressure distribution fared quite well. However, many features of the dimensionless normal force coefficients measured in the NASA-Ames wind tunnel tests are not modeled and predicted by the codes. In most cases, aerodynamic loads are estimated more accurately in the operating states below static stall. Since all wind turbines operating in the field will frequently experience significant yaw errors because of wind gusts and changing wind directions, these comparisons underscore the difficulty in accurately predicting extreme load cases.

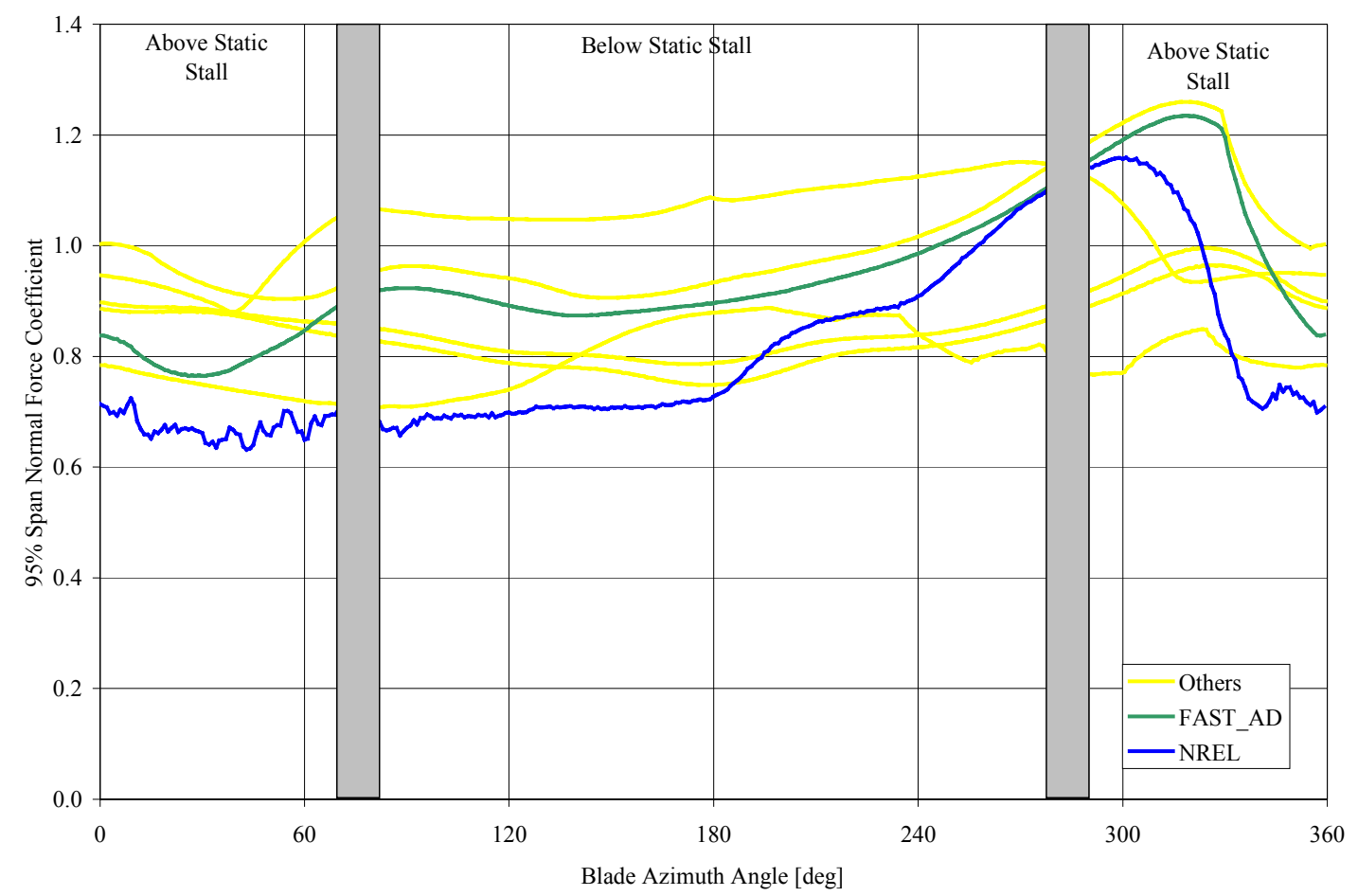

Figure 6.16: $95 \%$ span normal force coefficient, $15 \mathrm{~m} / \mathrm{s}$, upwind, $30^{\circ}$ yaw 


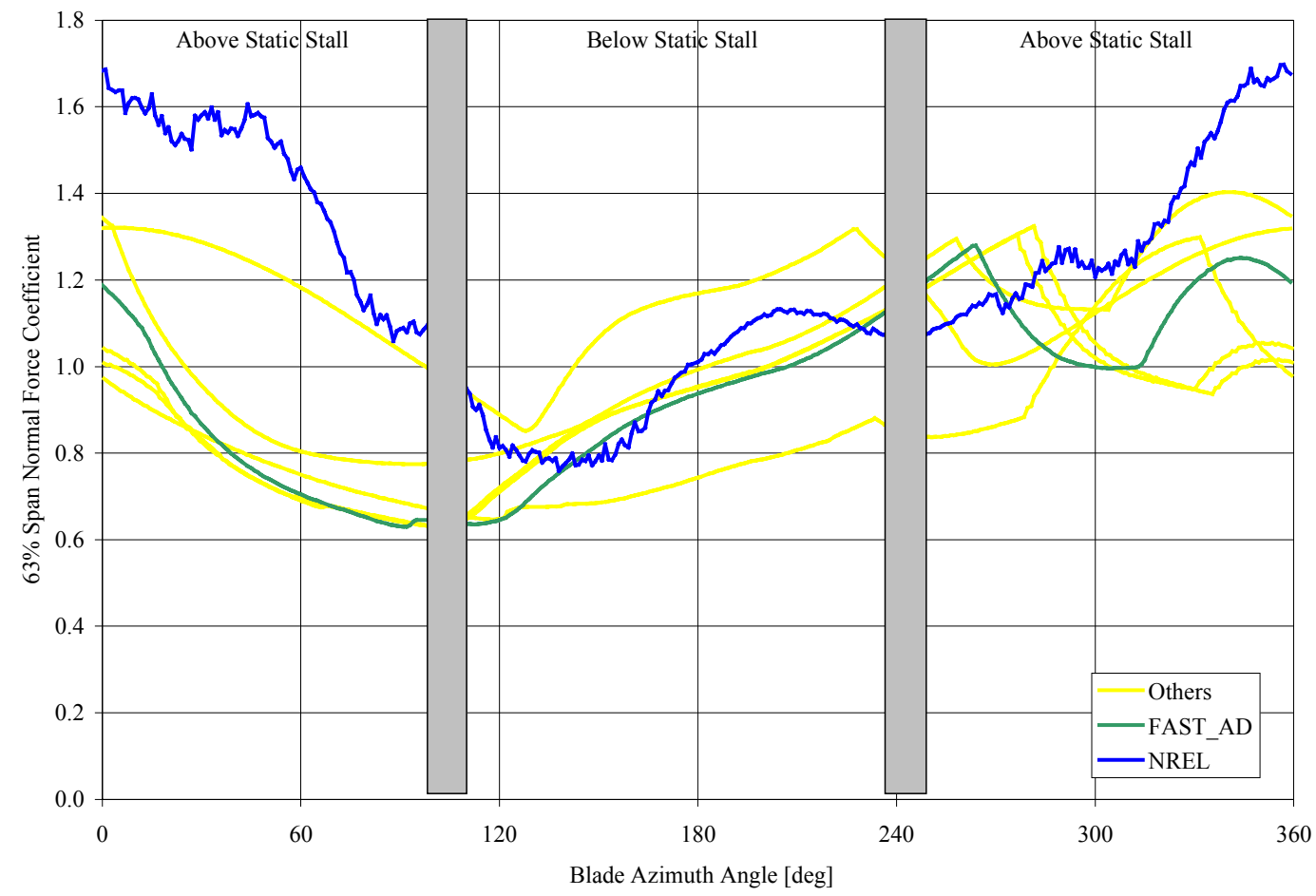

Figure 6.17: $63 \%$ span normal force coefficient, $15 \mathrm{~m} / \mathrm{s}$, upwind, $30^{\circ}$ yaw

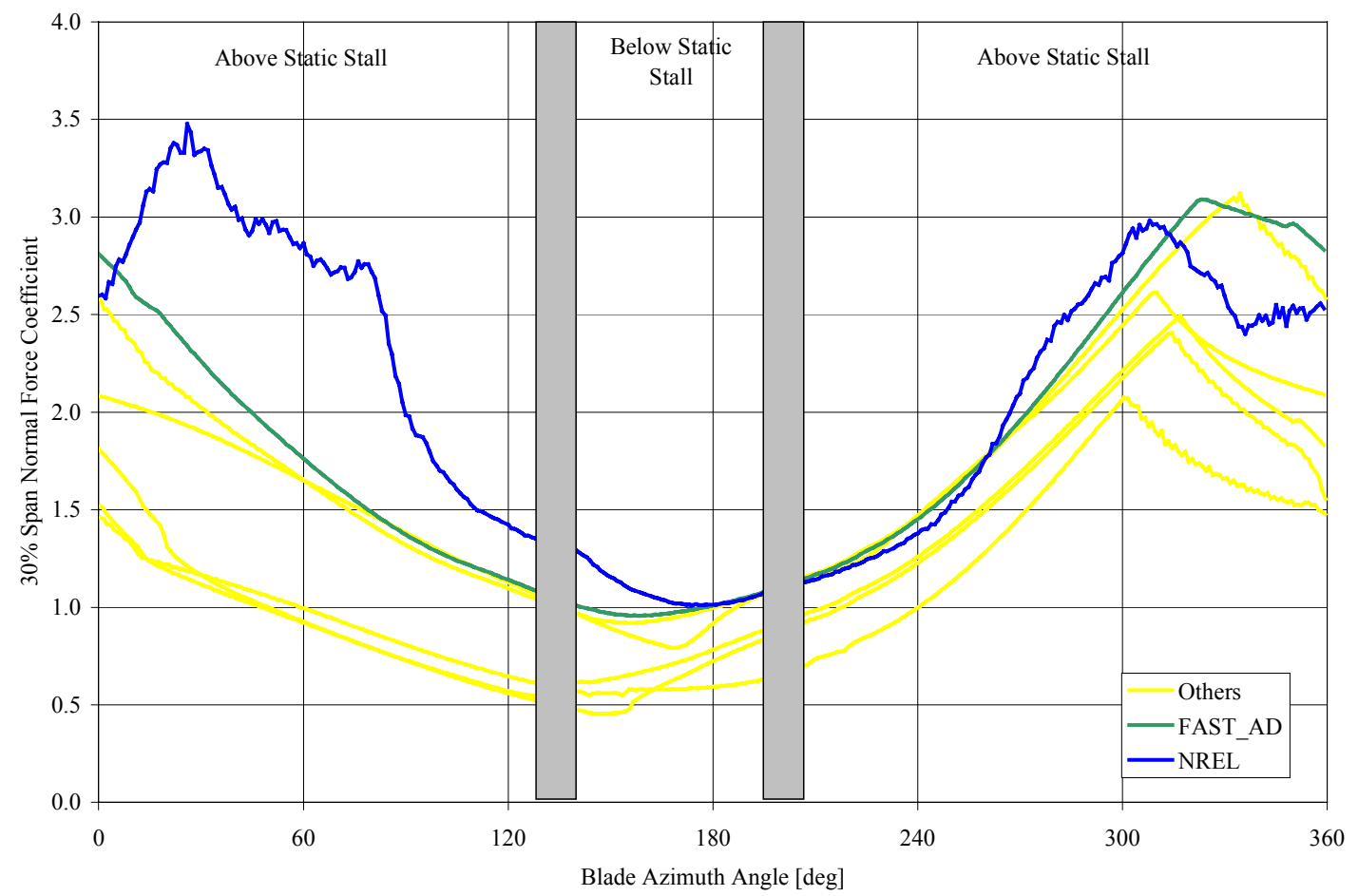

Figure 6.18: $30 \%$ span normal force coefficient, $15 \mathrm{~m} / \mathrm{s}$, upwind, $30^{\circ}$ yaw 


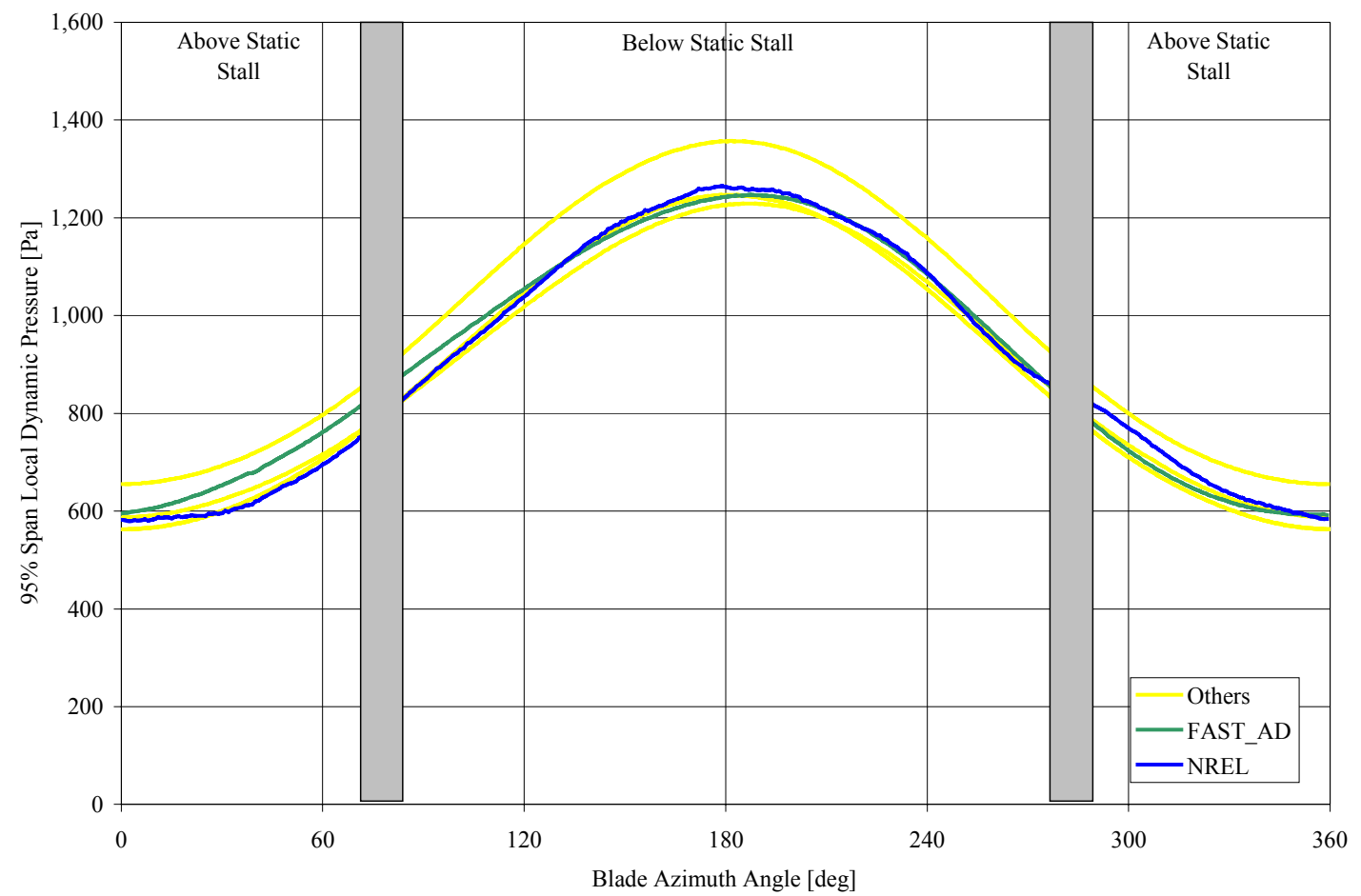

Figure 6.19: $95 \%$ span local dynamic pressure, $15 \mathrm{~m} / \mathrm{s}$, upwind, $30^{\circ}$ yaw

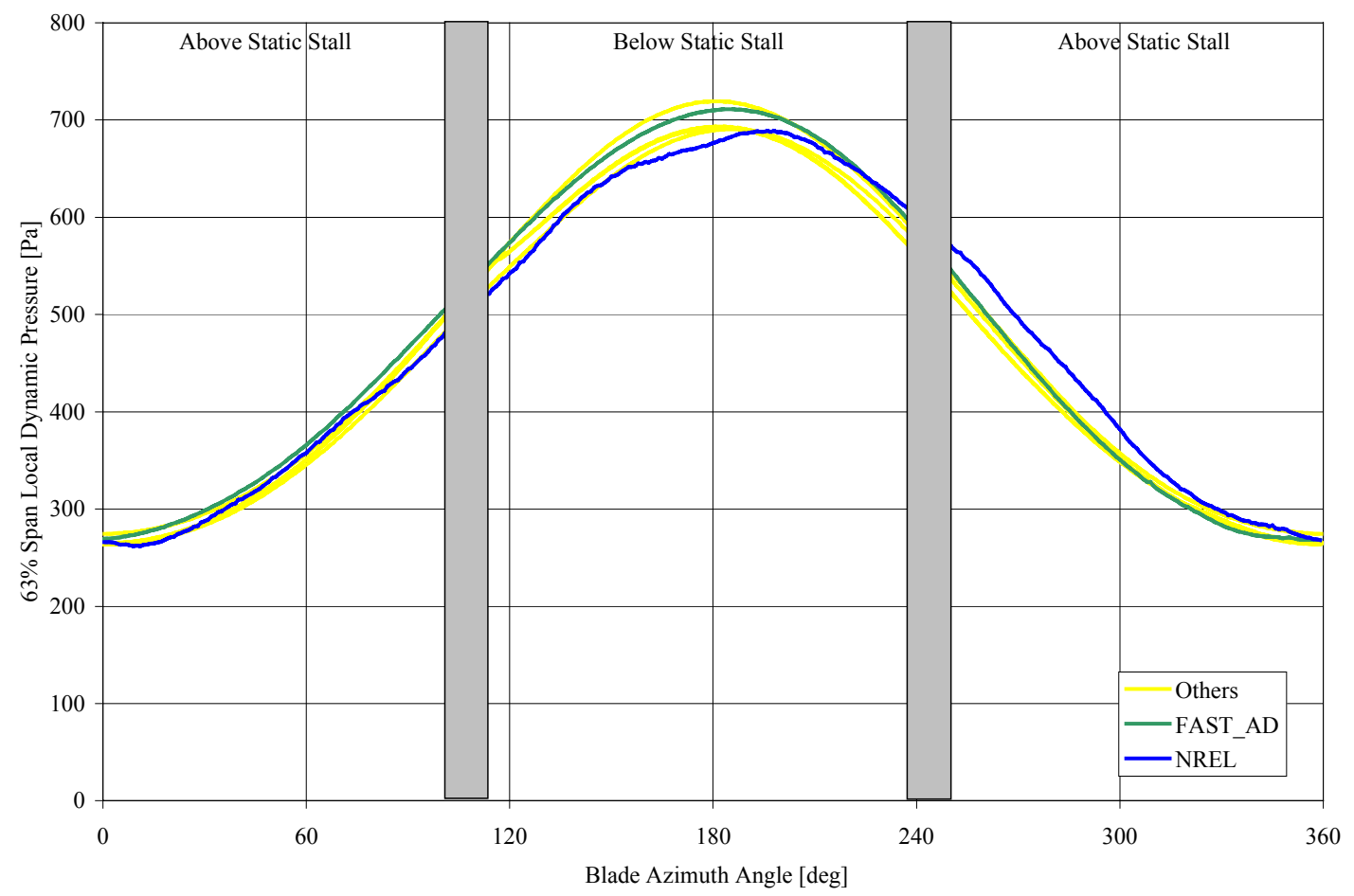

Figure 6.20: $63 \%$ span local dynamic pressure, $15 \mathrm{~m} / \mathrm{s}$, upwind, $30^{\circ}$ yaw 


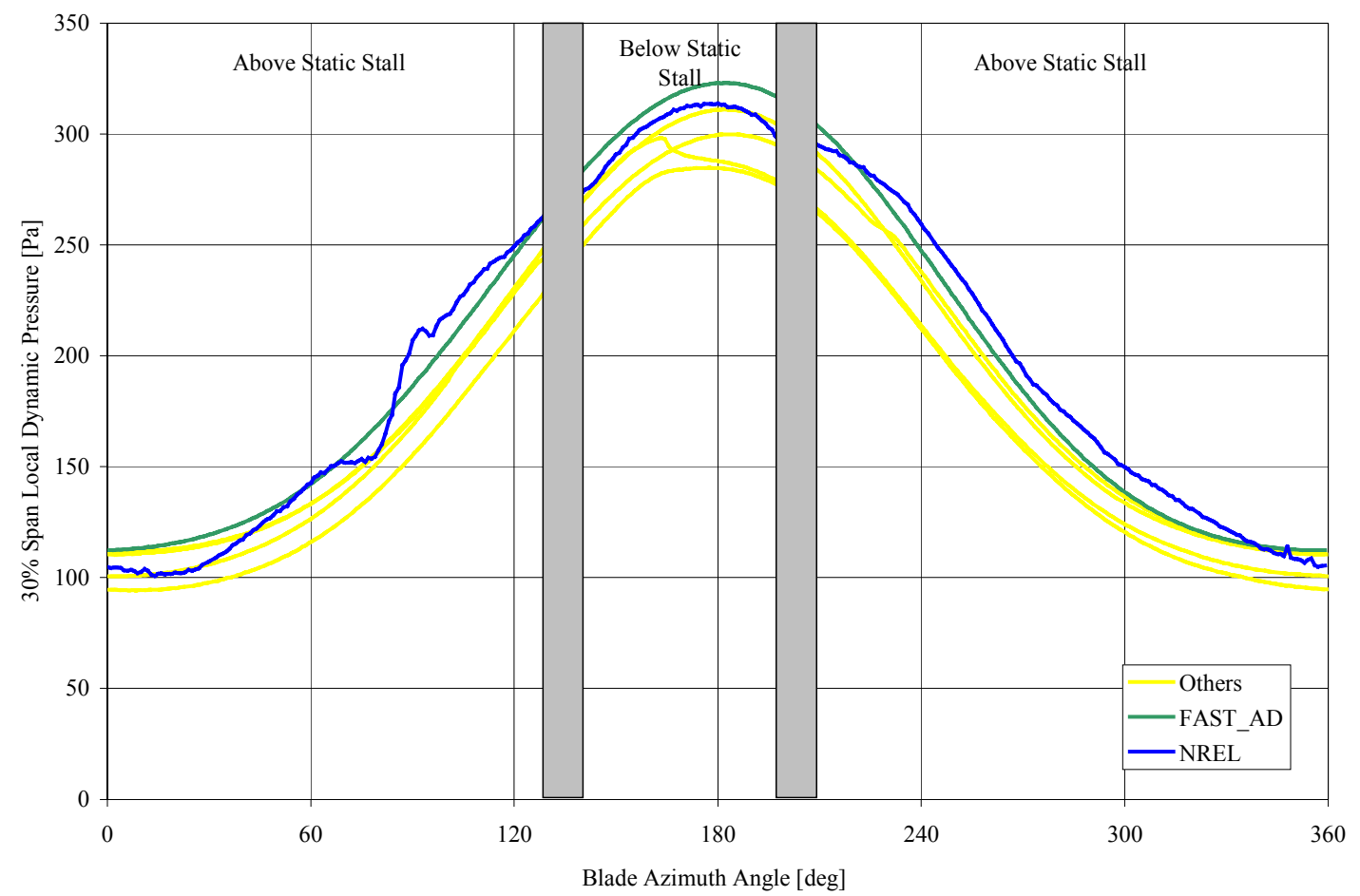

Figure 6.21 : $30 \%$ span local dynamic pressure, $15 \mathrm{~m} / \mathrm{s}$, upwind, $30^{\circ}$ yaw

No individual code predicted experimentally measured load and performance data consistently, or for several of the test cases or particular span stations. Discrepancies between the code predictions and the experimental test data should not be faulted on inaccuracies in the test data, since they are precise and repeatable (see section 5.2) and since these data often represent the average of the modelers' predictions. For the zero yaw error cases, load discrepancies also should not be faulted on the averaging that is done to obtain the load data for each wind speed. This is because the load spectra across azimuth angle orientation are fairly flat, especially for the low wind speed test cases (see Fig. 6.22). If there were tremendous jumps or spikes in the data, this last conclusion would be plausible. 


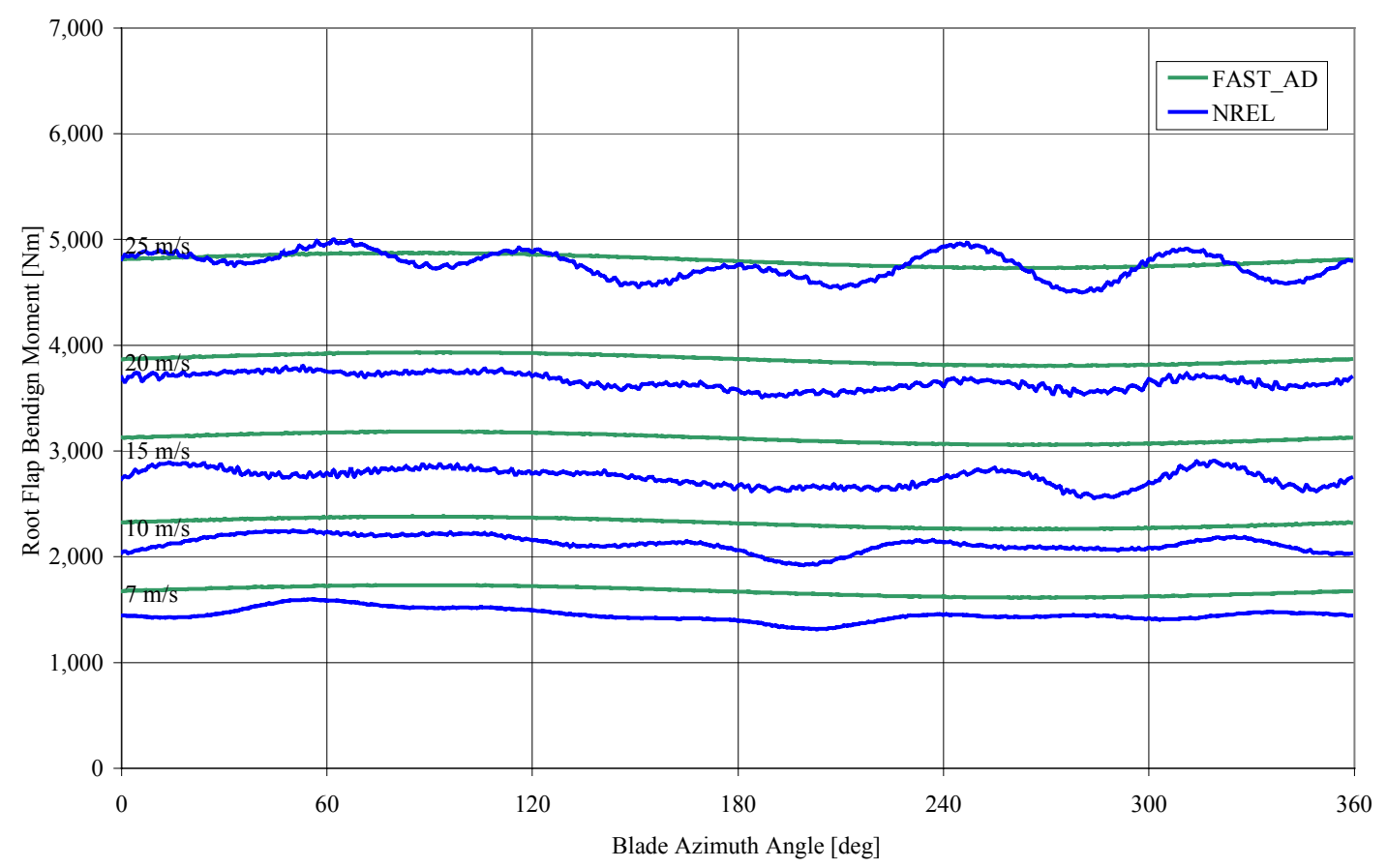

Figure 6.22: Root flap bending moment, upwind, zero yaw

In most instances, FAST_AD's code predictions also represent the average of the other modelers' predictions, revealing that the FAST_AD design code is comparable, if not somewhat better, at predicting loads and performances than other modeling tools that employ similar aerodynamics models. However, the accuracy of a modeling tool's predictions depends as much on how they are used (the modelers' tactics) as on the accuracy of the fundamental models and theories they employ. Most discrepancies between the load and performance predictions between the modelers are likely the results of choices made on how to use the turbine specifications outlined in section 5.1. Blind Comparison participants theorized, and the author agrees, that the choice of which aerodynamics data are used and the method, if any, of extrapolating these data to account for the effects of stall delay and deep stall explain for the most part large discrepancies between the aerodynamic force coefficient predictions and experimentally measured values. This last point will be demonstrated at the end of this chapter. The question of whether these large aerodynamic force coefficient discrepancies explain the large discrepancies in low-speed shaft torque and blade root flap bending moments is addressed in Chapter 7.

Other reasons for discrepancies between load and performance predictions between the modelers include the possibility of modeler error or code bugs. We did not scrutinize the work done by other modelers to determine what all these errors may be; however, the Blind Comparison documentation revealed several input deck inaccuracies. For instance, one Blind Comparison participant misinterpreted the definition of the ForcN and ForcT output channels of the blade element aerodynamic predictions. This participant erroneously assumed that these aerodynamic forces represent the differential forces normal and tangent to the element's chord line. In fact, these forces in AeroDyn represent the differential thrust and torque forces for blades that have no coning (see footnote 30 in section 6.1). This error clearly affects this modeler's predictions of the normal and tangential forces at each pressure tap grouping span station; it also subtly 
propagates into the predictions of the local dynamic pressures since the values of these parameters must be backed out of the available output channel data. Another Blind Comparison participant chose not to linearly interpolate between the two nodes surrounding the correct span stations where the pressure tap groupings are located and instead chose to use the closest possible node. The span station used for output aerodynamic data is off by more than $2 \%$ in one instance. Since the operational regimes and resulting aerodynamic properties depend strongly on the blade span location, inaccurate positioning of span stations where the aerodynamic properties are recorded is a source of considerable load and performance prediction discrepancy.

Known inaccuracies with the input parameters and models that are coupled to the simulations performed with FAST_AD, other than those already mentioned, include:

(1) The six inboard airfoil data tables that attempt to account for the effects of stall delay are not lined up to the exact span stations for which each airfoil data table is derived.

(2) In the pre-processing of the turbine specifications, numerous linear interpolations are made to obtain parameters necessary for the main FAST_AD input file. Examples of parameters that involve linear interpolations include linear interpolations to obtain chord, twist, and thickness distribution parameters. No effort is made to determine whether an enhanced curve fitting technique is applicable.

(3) In the post-processing of the output blade element data, numerous linear interpolations are made to determine aerodynamic properties at the proper pressure tap grouping span locations. Again, no effort is made to determine whether an enhanced curve fitting technique is applicable.

(4) Several assumptions and approximations are made when estimating the structural properties of the blades and tower and their corresponding natural mode shapes.

(5) Errors in the structural models employed by FAST_AD code (see Chapters 3 and 4) were not fixed, or even discovered, before the simulations were run.

The consequences of these inaccuracies should be evident from the previous discussion and in the analysis that follows here and in Chapter 7.

To determine whether most aerodynamic load discrepancies are the direct results of airfoil data table selection and extrapolation, the following analysis is performed. This analysis also highlights the weaknesses in the standard BEM model.

Figure 6.23 demonstrates a graphical BEM theory hand computation of the actual aerodynamic operating conditions at each pressure tap grouping span location for the upwind rotor configuration, $7 \mathrm{~m} / \mathrm{s}$ wind speed, zero yaw error (S0700000) test case. The curves labeled "FAST_AD Airfoil Data" illustrate the different dimensionless lift coefficient airfoil data used by FAST_AD at all blade elements. (The curves closest to the top of the plot represent the airfoil data at the innermost span stations caused by stall delay.) The curves labeled "Other Airfoil Data" illustrate all the other dimensionless lift coefficient airfoil data used by the other Blind Comparison participants who employed the AeroDyn aerodynamics subroutines in their Blind Comparison work. The curves labeled "BEM Equations" depict the feasible lift coefficient and angle of attack relations [Eq. (2.73)] at each pressure tap grouping span station as marked. The diamonds labeled "NREL $7 \mathrm{~m} / \mathrm{s}$ " depict the actual aerodynamic operating conditions (lift 
coefficient and angle of attack pairs) at the five span stations measured during the NASA-Ames wind tunnel tests. These angle of attack data were recorded in a different NASA-Ames test than the dimensionless lift coefficient data (specifically, H0700000 instead of S0700000). The angle of attack probes, which were used to measure the angle of attack values, were not installed on Blade 3 during the S-series tests so the probes would not disturb the flow around the blade and the resulting measurements of pressure and associated aerodynamic forces. Figure 6.24 gives a similar illustration for the upwind rotor configuration, $10 \mathrm{~m} / \mathrm{s}$ wind speed, zero yaw error (S1000000) test case.

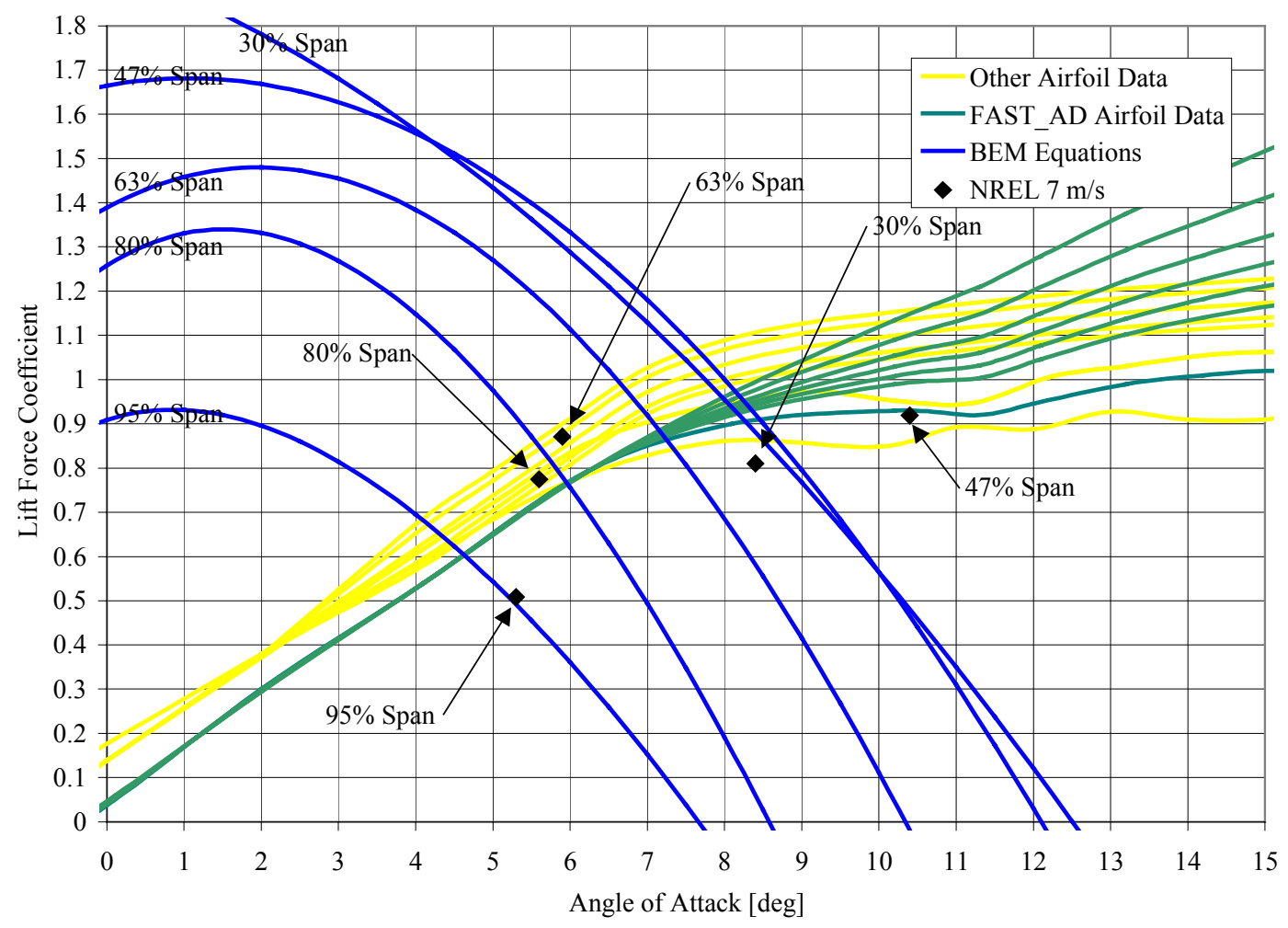

Figure 6.23: BEM theory hand computation, $7 \mathrm{~m} / \mathrm{s}$, upwind, zero yaw 


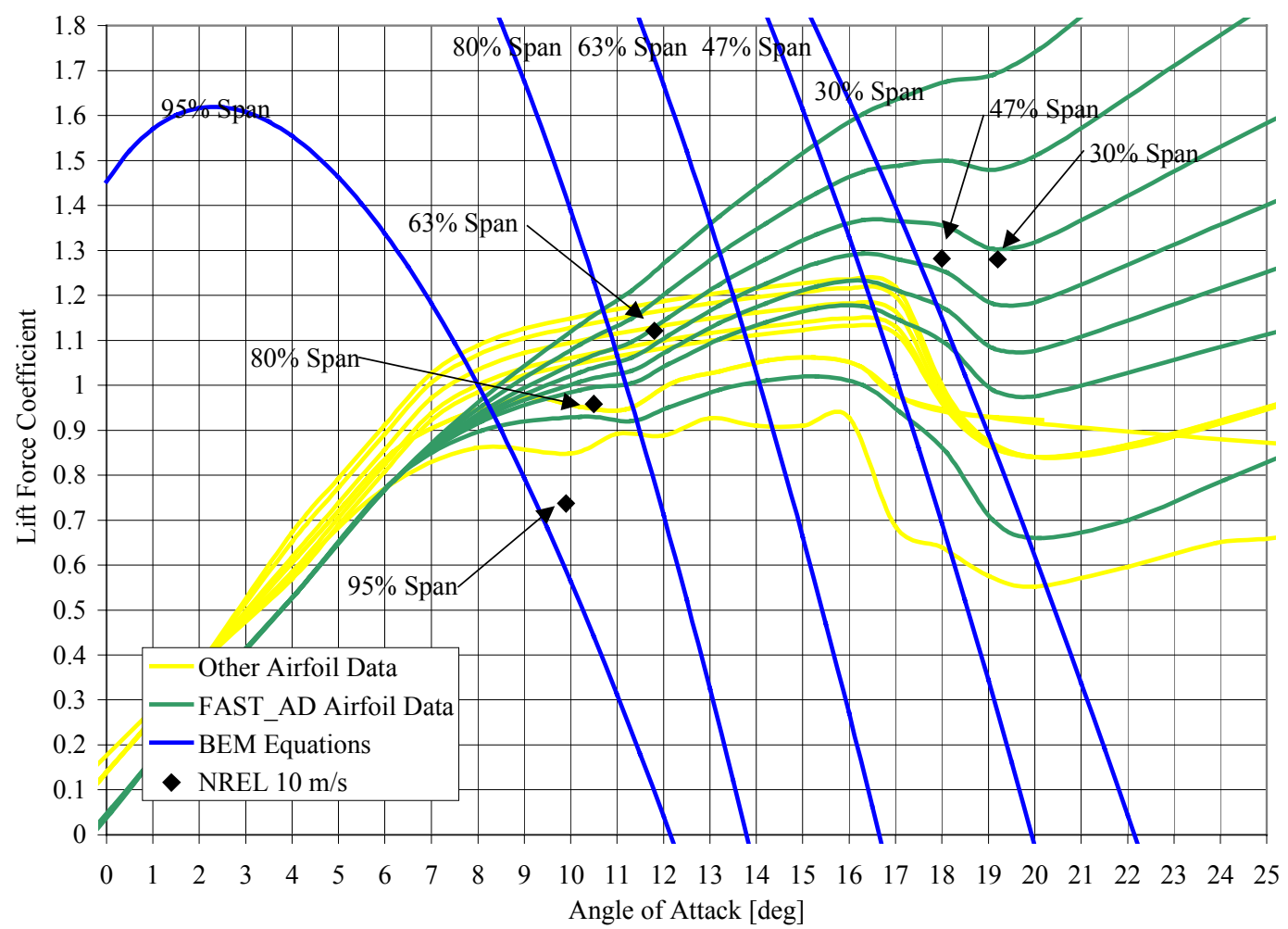

Figure 6.24: BEM theory hand computation, $10 \mathrm{~m} / \mathrm{s}$, upwind, zero yaw

As stated in section 2.5, according to BEM theory, the actual aerodynamic operating point (lift coefficient and angle of attack pair) at each span station is the intersection point between the associated BEM curve and the $C_{L}$ versus $\alpha$ curve characterizing the aerodynamic properties of the given airfoil. These BEM curves are independent of the blades' aerodynamic coefficients, and are thus equally valid for every dimensionless lift coefficient curve plotted in the figures.

Several conclusions may be drawn from these plots. First, the correlation between airfoil data selection spread and predicted load discrepancies is clear. The possible range of dimensionless lift coefficient values that could be predicted at a particular span station and wind speed using BEM theory is equal to the range of dimensionless lift coefficient airfoil data values that intersect the BEM curve at that particular span station and wind speed. For example, dimensionless lift coefficient airfoil data are $0.82-1.00$ for the pressure tap grouping located at $63.33 \%$ span at a wind speed of $7 \mathrm{~m} / \mathrm{s}$ (see Fig. 6.23). At this same span station and wind speed, the actual dimensionless normal force coefficient predictions were 0.82-1.04 (see Fig. 6.4). Since the normal and lift force coefficients are nearly equivalent for situations in which the angle of attack is small (as is the case here) [see Eq. (2.43)], the possible range of lift coefficient values almost entirely encapsulates the actual range predicted.

The fact that this actual range is not entirely encapsulated by the "possible" range is explainable by some of the inaccuracies previously discussed. For instance, when a span station used for aerodynamic load data recording is positioned imprecisely in a modeling tool, the BEM curve characterizing the aerodynamic operational state of that station is shifted from its corresponding nominal position. This in essence widens the range of possible dimensionless lift coefficient 
values that could be predicted at the associated nominal span station. The inaccuracy associated with linearly interpolating between adjoining span stations to determine the aerodynamic properties of a given pressure tap grouping at the proper span station is another explanation why the range of possible dimensionless lift coefficient values could be larger-even large enough to encapsulate the actual range predicted.

A summary of "possible" and actual ranges of dimensionless lift coefficient value predictions at the five pressure tap grouping span stations and upwind configuration, zero yaw error, 7 and 10 $\mathrm{m} / \mathrm{s}$ wind speed test cases is given in Table 6.10. The table shows that the likelihood the "possible" range encapsulates the actual range increases with increased wind speed, especially at the middle and innermost span stations.

Table 6.10: "Possible” and Actual Lift Coefficient Value Prediction Ranges

\begin{tabular}{|c|c|c|c|c|c|c|c|c|c|c|c|c|c|c|c|c|}
\hline \multirow{3}{*}{$\begin{array}{c}\text { Wind } \\
\text { Speed } \\
\mathrm{m} / \mathrm{s}\end{array}$} & & \multicolumn{15}{|c|}{ Blade Radial Location } \\
\hline & & \multicolumn{3}{|c|}{$95.00 \%$ Span } & \multicolumn{3}{|c|}{$80.00 \%$ Span } & \multicolumn{3}{|c|}{$63.33 \%$ Span } & \multicolumn{3}{|c|}{$46.67 \%$ Span } & \multicolumn{3}{|c|}{$30.00 \%$ Span } \\
\hline & & Min & & Max & Min & & $\operatorname{Max}$ & Min & & $\operatorname{Max}$ & Min & & Max & Min & & Max \\
\hline \multirow{2}{*}{7} & Possible & 0.60 & $\mid-$ & 0.70 & 0.76 & & 0.88 & 0.82 & L- & 1.00 & 0.86 & - & \begin{tabular}{|l|}
1.07 \\
\end{tabular} & 0.86 & - & 1.06 \\
\hline & Actual & 0.10 & - & 0.92 & 0.66 & - & 0.94 & 0.82 & - & 1.04 & 0.86 & - & 1.08 & 0.86 & - & 1.09 \\
\hline \multirow{2}{*}{10} & Possible & 0.85 & - & 1.08 & 0.88 & - & 1.18 & 0.90 & - & 1.36 & 0.62 & - & \begin{tabular}{|l|}
1.54 \\
\end{tabular} & 0.56 & - & 1.60 \\
\hline & Actual & 0.40 & - & 1.05 & 0.90 & - & 1.08 & 0.85 & - & 1.14 & 0.74 & - & 1.01 & 0.77 & - & 1.28 \\
\hline
\end{tabular}

The hand computations of these two test cases show why nearly everyone overpredicted normal force coefficients at the $95 \%$ span location. Figs. 6.23 and 6.24 show that the actual measured $C_{L}-\alpha$ aerodynamic operating points at this span station do not even lie within the range of used airfoil data. This indicates that three-dimensional flow effects prohibit the use of unmodified two-dimensional airfoil data at the outboard span stations. Moreover, Chapter 7 shows that no pressure tap grouping span stations exhibited the same two-dimensional aerodynamic characteristics measured in the CSU, OSU, and DUT wind tunnels. This reveals the flaws in assumptions (1) and (2) of blade element theory (see section 2.4). That is, the pressure distribution across a wind turbine blade during normal operation most likely causes threedimensional flow effects, such as spanwise flow, flow circulation, and vorticity. The nature of this flow has strong effect on the $C_{L}-\alpha$ characteristics of each blade element when contrasted with measurements taken in a two-dimensional flow environment (wind tunnel measurements made on static airfoils). If airfoil data used for modeling purposes are to be obtained from wind tunnel tests on static airfoils, new models that can extrapolate two-dimensional airfoil data to account for these three-dimensional flow effects are needed across the entire blade span. The only popular models that are used to extrapolate two-dimensional airfoil data to account for three-dimensional flow effects are stall delay models for the inboard span stations.

A final conclusion drawn from the hand computations is that modeling tool predictions of the angles of attack at the various span stations are erroneous in a noteworthy number of situations. The misprediction of angle of attack is especially critical around the linear portion of the $C_{L^{-}} \alpha$ curve and around stall. The slopes of the $C_{L}-\alpha$ curve in these operating regimes are considerably steeper than in other operating regimes, and thus, a slight misprediction in angle of attack can easily lead to a drastic misprediction in aerodynamic properties. The effects of this detail will be clearly seen in the sensitivity study of the next chapter. 
FAST_AD simulations use the dynamic inflow model instead of the standard BEM model. Nevertheless, these last few conclusions remain entirely valid, since the two theories predict nearly identical load and performance values for zero yaw error test cases - cases where dynamic stall events are minimal (Suzuki and Hansen 1998). This point is demonstrated further in the sensitivity study of Chapter 7. Several Blind Comparison participants who used the AeroDyn aerodynamics subroutines also used the dynamic inflow model during their simulations. 


\section{Sensitivity Study Input Parameters and Results}

Several causes of discrepancies between modeling tool load predictions are accounted for in Chapter 6 by taking into consideration some inconsistencies in the various input files. Additionally, weaknesses in the commonly used tip loss, stall delay, post stall, and other frequently used aerodynamic models are highlighted in Chapter 6 by examining discrepancies between load predictions and measured load values taken during the NASA-Ames wind tunnel tests. To further account for the many load prediction and measured load discrepancies observed in the Blind Comparison results, a sensitivity analysis is performed and discussed in this chapter. The sensitivity study is guided by the following questions:

(1) What are some factors that did not contribute to the load prediction discrepancies?

(2) What are the critical parameters that affect wind turbine load and performance prediction accuracy and could they have had an impact on the Blind Comparison outcome?

(3) Do the discrepancies in predicted and measured aerodynamic force coefficient values account for the large discrepancies in low-speed shaft torque and blade root flap bending moments? If not, what are other causes and what can be done to fix them?

(4) To have the greatest impact on wind turbine design improvement and wind-generated electricity cost reduction, where do ongoing wind turbine research programs need to concentrate?

To address these questions, a sample of the FAST_AD model inputs is systematically perturbed to determine its effect on load and performance predictions. Unfortunately, time prohibits the study of the effects of all realizable input parameter perturbations; focus is given to aerodynamic parameters, since aerodynamic forces are the key factors that determine wind turbine component loads. Moreover, since the dynamical events associated with complicated operational states cannot be studied before discrepancies in the simplest test cases are first understood, attention is given solely to upwind rotor configuration, zero yaw error test cases. Descriptions and reasons for each sensitivity analysis performed and the FAST_AD input file parameter alterations needed to perform these analyses are given in section 7.1. (The steps taken to develop the original FAST_AD input file parameters are outlined in section 6.1.) The results of each sensitivity analysis are compared and discussed in section 7.2. Only results that are above and beyond those established in Chapter 6 are discussed. Questions 1-4 are addressed wherever applicable.

\subsection{Sensitivity Studies and FAST_AD Input Parameter Alterations}

Eleven sensitivity analyses are chosen and simulated in FAST_AD for the six upwind configuration, zero yaw error, $7,10,13,15,20$, and $25 \mathrm{~m} / \mathrm{s}$ (S**00000-series) test cases. The names of each study and the differences between their input file(s) and the original file(s) used for the for Blind Comparison exercise are summarized in Table 7.1. 
Table 7.1: Sensitivity Study Input Parameter Alterations for FAST_AD

\begin{tabular}{|c|c|c|c|c|}
\hline \multirow[b]{2}{*}{ Study Name } & \multicolumn{2}{|c|}{ Input Parameter(s) Modified } & \multicolumn{2}{|c|}{ Alteration(s) Made } \\
\hline & Variable & Description & From & To \\
\hline EQUIL & & Dynamic inflow model switch & DYNIN - Dynamic inflow model & $\begin{array}{l}\text { EQUIL - Equilibrium, standard } \\
\text { BEM model }\end{array}$ \\
\hline IZD40 & $I Z D(4)$ & Azimuth angle, rotor side switch & 1 - Induction generator & 0 - Constant speed \\
\hline DT001 & $D T$ & Time increment & 0.002 second & 0.001 second \\
\hline \multirow{3}{*}{ NR10 } & $N R$ & Number of blade elements & 20 - maximum allowable & 10 \\
\hline & NFOIL & Airfoil table ID numbers distributed across blade & \multirow{2}{*}{$\begin{array}{l}\text { Most well suited node given the } \\
\text { number of elements }\end{array}$} & \multirow{2}{*}{$\begin{array}{l}\text { Most well suited node given the } \\
\text { number of elements }\end{array}$} \\
\hline & PRIN & T / NOPRINT flags - Distributed across blade & & \\
\hline THETA3 & THETA & Blade collective pitch angle & 4.815 deg -3.0 deg pitch at tip & $3.0 \mathrm{deg}-1.185 \mathrm{deg}$ pitch at tip \\
\hline \multirow{2}{*}{ BETA34 } & BETA $(1)$ & Blade 1 coning angle & \multirow{2}{*}{$0.0 \mathrm{deg}$ - No preconing } & \multirow{2}{*}{$\begin{array}{l}3.4 \text { deg - same as downwind } \\
\text { configuration test cases }\end{array}$} \\
\hline & BETA(2) & Blade 2 coning angle & & \\
\hline RHO10PL & $\mathrm{RHO}$ & Air density & Exact NASA-Ames test values & $10 \%$ less than these values \\
\hline \multirow{4}{*}{ PhaseVModeShapes } & $\mu_{B}$ & Distributed lineal density of blade & \multirow{4}{*}{$\begin{array}{l}\text { Properties of the tapered, twisted, UAE } \\
\text { blade }\end{array}$} & \multirow{4}{*}{$\begin{array}{l}\text { Properties of the constant-chord, } \\
\text { highly twisted blade used during } \\
\text { Phase V testing }\end{array}$} \\
\hline & $E I$ & Distributed flapwise stiffness across blade & & \\
\hline & $E I$ & Distributed edgewise stiffness across blade & & \\
\hline & $P C(* *)$ & Blade mode shape const. proportionality coef.s & & \\
\hline NegTHETAS & $\theta_{S}$ & Distributed structural pretwist across blade & $\begin{array}{l}\text { Properties of the tapered, twisted, UAE } \\
\text { blade }\end{array}$ & Negative of these values \\
\hline \multirow{3}{*}{ NASAAmesAirfoilData } & NUMFOIL & Number of different airfoil tables & 8 & 6 \\
\hline & \multicolumn{2}{|r|}{ Airfoil data table files } & $\begin{array}{l}\text { OSU data at } \operatorname{Re}=750,000 \text { extrapolated } \\
\text { for stall delay at } 12.9 \%, 18.5 \%, 24.2 \% \text {, } \\
29.8 \%, 35.4 \% \text {, and } 41.0 \% \text { span }\end{array}$ & $\begin{array}{l}\text { Effective NASA-Ames test 2D } \\
\text { airfoil data at } 30.0 \%, 46.7 \% \text {, } \\
63.3 \%, 80.0 \% \text {, and } 95.0 \% \text { span } \\
\end{array}$ \\
\hline & NFOIL & Airfoil table ID numbers distributed across blade & $\begin{array}{l}\text { Most well suited node given the } \\
\text { number of elements }\end{array}$ & $\begin{array}{l}\text { Most well suited node given the } \\
\text { number of elements }\end{array}$ \\
\hline \multirow{5}{*}{ NASAAmesAlpha } & NUMFOIL & Number of different airfoil tables & 8 & 6 \\
\hline & \multicolumn{2}{|r|}{ Airfoil data table files } & $\begin{array}{l}\text { OSU data at } \operatorname{Re}=750,000 \text { extrapolated } \\
\text { for stall delay at } 12.9 \%, 18.5 \%, 24.2 \% \text {, } \\
29.8 \%, 35.4 \% \text {, and } 41.0 \% \text { span }\end{array}$ & $\begin{array}{l}\text { Effective NASA-Ames test 2D } \\
\text { airfoil data at } 30.0 \%, 46.7 \% \text {, } \\
63.3 \%, 80.0 \% \text {, and } 95.0 \% \text { span } \\
\end{array}$ \\
\hline & NFOIL & Airfoil table ID numbers distributed across blade & $\begin{array}{l}\text { Most well suited node given the } \\
\text { number of elements }\end{array}$ & $\begin{array}{l}\text { Most well suited node given the } \\
\text { number of elements }\end{array}$ \\
\hline & $\overline{\theta_{T}}$ & Distributed aerodynamic pretwist across blade & \multirow{2}{*}{$\begin{array}{l}\text { Properties of the tapered, twisted, UAE } \\
\text { blade }\end{array}$} & \multirow{2}{*}{$\begin{array}{l}\text { Adjusted at each span location } \\
\text { by the average discrepancy }\end{array}$} \\
\hline & $\theta_{S}$ & Distributed structural pretwist across blade & & \\
\hline
\end{tabular}

The sensitivity study titled "EQUIL" is performed to demonstrate the equivalency of the dynamic inflow and classic BEM models when simulating zero yaw error test scenarios (operating scenarios in which dynamic stall events are minimal). The standard BEM model is selected by designating the "EQUIL" flag for the dynamic inflow model switch parameter instead of the "DYNIN" flag in the main FAST_AD input file.

The modelers who used the AeroDyn aerodynamics subroutines during their Blind Comparison work employed several induction generator models (including constant speed or no generator model). To verify whether the induction generator model employed by FAST_AD has any impact on the resulting load predictions, the sensitivity study titled "IZD40" is carried out. In these simulations, the rotor is modeled with a constant speed instead of as an induction generator with a fixed linear generator constant.

To determine whether the time step used in FAST_AD for the Blind Comparison work is too large and affected the load predictions, the test cases are rerun with a time step half the size of the original $(0.001$ instead of $0.002 \mathrm{~s})$. This is done in the sensitivity study titled "DT001."

Of similar merit, the accuracy of the model to the number of blade elements for integrating aerodynamic and elastic forces is studied in the analysis titled "NR10." This study chooses half as many blade elements as were chosen originally (10 instead of the maximum of 20). Aerodynamic characteristics associated with each of the 11 new blade nodes are listed in Table 7.2. This study also examines the sensitivity of the load predictions to the linear interpolations 
that are performed to determine the aerodynamic properties at the correct pressure tap grouping span locations. Detailed instantaneous aerodynamic data for determining these aerodynamic properties are requested at nodes $3,4,5,7,9,10$, and 11 . In addition to the input file alterations listed in Table 7.1, the input file for the post-processing code Crunch had to be extensively modified to accommodate these changes.

Table 7.2: Nodal Aerodynamic Properties for Sensitivity Study “NR10”

\begin{tabular}{|c|c|c|c|c|c|c|c|c|}
\hline \multirow[b]{2}{*}{$\begin{array}{c}\text { Node } \\
\text { Number } \\
\end{array}$} & \multicolumn{4}{|c|}{ Node Location } & \multirow{2}{*}{\begin{tabular}{|c|} 
Prescribed \\
Airfoil \\
Data \\
Table \\
Identifier \\
\end{tabular}} & \multirow[b]{2}{*}{$\begin{array}{r}\text { Local } \\
\text { Chord } \\
\text { Length }(c) \\
m \\
\end{array}$} & \multirow[b]{2}{*}{$\begin{array}{r}\text { Local } \\
\text { Twist }\left(\theta_{T}\right) \\
d e g \\
\end{array}$} & \multirow[b]{2}{*}{$\begin{array}{r}\text { Local } \\
\text { Thickness } \\
m\end{array}$} \\
\hline & $\begin{array}{l}\text { Distance } \\
\text { from Rotor } \\
\text { Center } \\
m\end{array}$ & $\begin{array}{l}\text { Spanwise } \\
\text { Fraction } \\
(/ 5.029 \mathrm{~m})\end{array}$ & $\begin{array}{l}\text { Distance } \\
\text { Along Blade } \\
\text { From Root }(r) \\
m\end{array}$ & $\begin{array}{l}\text { Radial } \\
\text { Fraction } \\
(r / 4.597 \mathrm{~m})\end{array} \mid$ & & & & \\
\hline 1 & 0.432 & 0.086 & 0.000 & 0.000 & 1 & 0.218 & 0.00 & 1.000 \\
\hline 2 & 0.892 & 0.177 & 0.460 & 0.100 & 3 & \begin{tabular}{|c|}
0.283 \\
\end{tabular} & 3.66 & 0.707 \\
\hline 3 & 1.351 & 0.269 & 0.919 & 0.200 & 4 & 0.727 & 17.89 & 0.209 \\
\hline 4 & 1.811 & 0.360 & 1.379 & 0.300 & 6 & 0.680 & 10.19 & 0.209 \\
\hline 5 & 2.271 & 0.452 & 1.839 & 0.400 & 8 & 0.634 & 5.52 & 0.209 \\
\hline 6 & 2.731 & 0.543 & 2.299 & 0.500 & 8 & 0.588 & 2.81 & 0.209 \\
\hline 7 & 3.190 & 0.634 & 2.758 & 0.600 & 8 & 0.542 & 1.21 & 0.209 \\
\hline 8 & 3.650 & 0.726 & 3.218 & 0.700 & 8 & 0.495 & 0.21 & 0.209 \\
\hline 9 & 4.110 & 0.817 & 3.678 & 0.800 & 8 & 0.448 & -0.51 & 0.209 \\
\hline 10 & 4.569 & 0.909 & 4.137 & 0.900 & 8 & 0.402 & -1.17 & 0.209 \\
\hline 11 & 5.029 & 1.000 & 4.597 & 1.000 & 8 & 0.355 & -1.82 & 0.209 \\
\hline
\end{tabular}

Defining the blade collective pitch angle is problematic. Many wind turbine analysts believe it should be defined with respect to the local aerodynamic twist at $75 \%$ blade span; others believe it should defined with respect to the zero twist location (which may or may not be at $75 \%$ blade span); still others believe it should defined with respect to the blade tip. Several Blind Comparison participants may have used $3^{\circ}$ instead of $4.815^{\circ}$ for the blade collective pitch angle at zero aerodynamic twist $\left(3^{\circ}\right.$ is the actual angle between the tip chord line and rotor plane, regarded as the blade pitch angle for all NASA-Ames test cases chosen for the Blind Comparison exercise). The sensitivity study titled "THETA3" shows how this error will propagate through the simulated load predictions. To do this, the FAST_AD input parameter THETA is set fixed at $3^{\circ}$ instead of $4.815^{\circ}$, corresponding to an angle between the tip chord line and rotor plane of $1.185^{\circ}$ (an angle of $1.815^{\circ}$ smaller than it should be). This study additionally highlights the sensitivity of the model to blade torsional motion since aerodynamic responses to any significant torsional motion should be at least the same order of magnitude as the responses to rather small pitch angle perturbations. In addition to the input file alteration listed in Table 7.1, the input file for the post-processing code Crunch had to be modified extensively to accommodate this change.

Similar to "THETA3," the sensitivity study titled "BETA34" is performed to demonstrate how sensitive the aerodynamic models are to coning angle perturbations and how an error made in the blade coning angles affects the load predictions. In this study, the coning angle of each blade for the upwind rotor configuration is fixed at $3.4^{\circ}$, the coning angle used for the downwind rotor configuration test cases. (Downwind preconing for upwind rotor configurations in FAST_AD is modeled using positive precone angles.) Though it is doubtful that any Blind Comparison participant made this error, this study additionally demonstrates the sensitivity of the model to blade flapping motion, since aerodynamic responses to any significant flapping motion should be at least the same order of magnitude as the responses to rather small precone angle perturbations. 
The sensitivity analysis titled "RHO10PL" demonstrates how an underprediction in air density is reflected in an underprediction of the potential loads. This is done by reducing the exact NASAAmes test values by $10 \%$ for each of the six test cases considered for this study. This study has less to do with load prediction discrepancy explanations and more to do with wind turbine design considerations. For example, it is important to consider how the potential loads may change if a wind turbine designed reflecting conditions seen at relatively high altitudes (say Colorado) is installed at a lower altitude (say sea level).

Since several assumptions and approximations were made to estimate the structural properties of the blades of the UAE used during Phase VI (see section 6.1) a sensitivity analysis is performed to determine whether any structural property inaccuracies contributed to any of the load prediction inaccuracies. To accomplish this, structural properties of the UAE Phase V blades are input into FAST_AD in the sensitivity study titled "PhaseVModeShapes." The structural properties and mode shapes of the Phase V blades are considerably different than those used for the Phase VI testing. If differences in the load predictions are minor, inaccuracies in the structural properties had a negligible impact on load prediction inaccuracies. If not, inaccuracies in the blade structural properties probably played a role in the load prediction inaccuracies. A summary of the calibrated distributed structural properties of the UAE Phase V blades is given in Table 6.4. To obtain the proper mode shapes needed for the FAST_AD input file (local flapwise and edgewise blade mode shapes instead of out-of-plane and in-plane mode shapes) the distributed structural pretwist is zeroed out in the Modes input file and Modes is run again. Centrifugal stiffening effects are accounted for in this process by specifying a typical rotor rotational speed of $72 \mathrm{rpm}$. A summary of the mode shapes is given in Table 7.3.

Table 7.3: Mode Shapes of the UAE Phase V Blades

\begin{tabular}{|c|c|r|r|r|}
\hline \multirow{2}{*}{$\begin{array}{c}\text { Shape } \\
\text { Function } \\
\text { Number }(b)\end{array}$} & $\begin{array}{c}\text { Shape } \\
\text { Function } \\
\left(\varphi_{b} \text { 's }\right)\end{array}$ & $\begin{array}{r}|c| \\
1^{\text {st }} \text { Mode Const. } \\
\text { Proportionality } \\
\text { Coef.s }\left(C_{l, b} \text { 's }\right)\end{array}$ & $\begin{array}{r}2^{\text {nd }} \text { Mode Const. } \\
\text { Proportionality } \\
\text { Coef.s }\left(C_{2, b} \text { 's }\right)\end{array}$ & $\begin{array}{r}1^{\text {st }} \text { Edgewise } \\
\text { Mode Const. } \\
\text { Proportionality } \\
\text { Coef.s }\left(C_{l, b} \text { 's }\right)\end{array}$ \\
\hline 2 & {$\left[r /\left(R-R_{H}\right)\right]^{2}$} & 0.225 & -0.795 & 0.931 \\
\hline 3 & {$\left[r /\left(R-R_{H}\right)\right]^{3}$} & 0.155 & -10.547 & 0.569 \\
\hline 4 & {$\left[r /\left(R-R_{H}\right)\right]^{4}$} & 4.728 & 17.341 & 0.057 \\
\hline 5 & {$\left[r /\left(R-R_{H}\right)\right]^{5}$} & -6.612 & 0.487 & -1.094 \\
\hline 6 & {$\left[r /\left(R-R_{H}\right)\right]^{6}$} & 2.503 & -5.485 & 0.536 \\
\hline
\end{tabular}

The sensitivity study titled "NegTHETAS" is performed to determine how a properly oriented structural pretwist affects the resulting load predictions. That is, this study counteracts the error in the structural model indicated in footnote 9 of Chapter 3 by reversing the sign of each distributed structural pretwist entry in the main FAST_AD input file.

As mentioned in Chapter 6, none of the pressure tap grouping span stations exhibit the same twodimensional aerodynamic characteristics measured in the CSU, OSU, and DUT wind tunnels. The validity of this statement is seen in the data that follow. The aerodynamic force coefficient- 
angle of attack relationships at each pressure tap grouping span station (as witnessed in the threedimensional environment of the NASA-Ames wind tunnel tests) are given in Tables 7.4-7.8 and are graphically illustrated in Figs. 7.1-7.4. That is, these data (labeled "NREL **.*\%" in the figures) reflect the operational environment of the wind turbine; the wind turbine was operating when each data point was recorded. Each data point represents the average force coefficient and angle of attack pairs measured at a single wind speed for the upwind configuration, zero yaw error test cases. (The data points associated with the smallest angles of attack correspond to data measured during the smallest wind speed test cases.) The angle of attack data were recorded in different NASA-Ames tests than the dimensionless lift coefficient data (specifically, $\mathrm{H}^{* *} 00000$ series instead of $\mathrm{S}^{* *} 00000$-series). To prevent the angle of attack probes, which were used to measure the angle of attack values, from disturbing the flow around the blade and the resulting measurements of pressure and associated aerodynamic forces, these probes were not installed on Blade 3 during the S-series tests.

Table 7.4: NREL S809 Airfoil Data from NASA-Ames Tests: 95\% Span

\begin{tabular}{|l|c|r|r|r|r|}
\hline $\begin{array}{l}\text { Wind } \\
\text { Speed } \\
m / s\end{array}$ & $\begin{array}{r}\text { Angle of } \\
\text { Attack }(\alpha) \\
d e g\end{array}$ & $\begin{array}{r}\text { Dim.less } \\
\text { Normal } \\
\text { Coeff. }\left(C_{N}\right)\end{array}$ & $\begin{array}{r}\text { Dim.less } \\
\text { Tangential } \\
\text { Coeff. }\left(C_{T}\right)\end{array}$ & $\begin{array}{r}\text { Dim.less } \\
\text { Lift Coeff. } \\
\left(C_{L}\right)\end{array}$ & $\begin{array}{r}\text { Dim.less } \\
\text { Drag Coeff. } \\
\left(C_{D}\right)\end{array}$ \\
\hline 5 & 2.6 & 0.3467 & 0.0059 & 0.3466 & 0.0098 \\
\hline 6 & 4.0 & 0.4268 & 0.0221 & 0.4273 & 0.0077 \\
\hline 7 & 5.3 & 0.5065 & 0.0414 & 0.5082 & 0.0056 \\
\hline 8 & 6.8 & 0.6200 & 0.0624 & 0.6230 & 0.0114 \\
\hline 9 & 8.4 & 0.6870 & 0.0811 & 0.6915 & 0.0201 \\
\hline 10 & 9.9 & 0.7311 & 0.0993 & 0.7373 & 0.0279 \\
\hline 11 & 11.3 & 0.7430 & 0.1145 & 0.7510 & 0.0333 \\
\hline 12 & 12.8 & 0.7592 & 0.1325 & 0.7697 & 0.0390 \\
\hline 13 & 13.9 & 0.7969 & 0.1463 & 0.8087 & 0.0494 \\
\hline 14 & 15.1 & 0.8104 & 0.1492 & 0.8213 & 0.0671 \\
\hline 15 & 16.1 & 0.7849 & 0.1391 & 0.7927 & 0.0840 \\
\hline 16 & 17.7 & 0.7550 & 0.1012 & 0.7500 & 0.1331 \\
\hline 17 & 18.9 & 0.7282 & 0.0458 & 0.7038 & 0.1925 \\
\hline 18 & 20.1 & 0.7386 & -0.0112 & 0.6898 & 0.2643 \\
\hline 19 & 21.4 & 0.7597 & -0.0233 & 0.6988 & 0.2989 \\
\hline 20 & 22.5 & 0.7147 & -0.0565 & 0.6387 & 0.3257 \\
\hline 21 & 23.9 & 0.6499 & -0.0621 & 0.5690 & 0.3201 \\
\hline 22 & 24.9 & 0.6298 & -0.0622 & 0.5451 & 0.3216 \\
\hline 23 & 26.0 & 0.6319 & -0.0627 & 0.5405 & 0.3334 \\
\hline 24 & 26.9 & 0.6077 & -0.0615 & 0.5141 & 0.3298 \\
\hline 25 & 27.8 & 0.6061 & -0.0616 & 0.5074 & 0.3372 \\
\hline
\end{tabular}


Table 7.5: NREL S809 Airfoil Data from NASA-Ames Tests: $80 \%$ Span

\begin{tabular}{|l|r|r|r|r|r|}
\hline $\begin{array}{l}\text { Wind } \\
\text { Speed } \\
m / s\end{array}$ & $\begin{array}{r}\text { Angle of } \\
\text { Attack }(\alpha) \\
\text { deg }\end{array}$ & $\begin{array}{r}\text { Dim.less } \\
\text { Normal } \\
\text { Coeff. }\left(C_{N}\right)\end{array}$ & $\begin{array}{r}\text { Dim.less } \\
\text { Tangential } \\
\text { Coeff. }\left(C_{T}\right)\end{array}$ & $\begin{array}{r}\text { Dim.less } \\
\text { Lift Coeff. } \\
\left(C_{L}\right)\end{array}$ & $\begin{array}{r}\text { Dim.less } \\
\text { Drag Coeff. } \\
\left(C_{D}\right)\end{array}$ \\
\hline 5 & 2.6 & 0.4787 & 0.0167 & 0.4790 & 0.0050 \\
\hline 6 & 4.1 & 0.6108 & 0.0433 & 0.6123 & 0.0005 \\
\hline 7 & 5.6 & 0.7709 & 0.0730 & 0.7743 & 0.0026 \\
\hline 8 & 7.2 & 0.8534 & 0.1015 & 0.8594 & 0.0063 \\
\hline 9 & 8.9 & 0.8788 & 0.1232 & 0.8873 & 0.0142 \\
\hline 10 & 10.5 & 0.9474 & 0.1483 & 0.9586 & 0.0268 \\
\hline 11 & 12.1 & 1.0202 & 0.1672 & 1.0326 & 0.0504 \\
\hline 12 & 13.6 & 0.9857 & 0.1653 & 0.9969 & 0.0711 \\
\hline 13 & 14.8 & 1.0228 & 0.1516 & 1.0276 & 0.1147 \\
\hline 14 & 16.1 & 1.0591 & 0.0493 & 1.0312 & 0.2463 \\
\hline 15 & 17.3 & 0.9184 & -0.0015 & 0.8764 & 0.2745 \\
\hline 16 & 18.8 & 0.9924 & -0.0141 & 0.9349 & 0.3332 \\
\hline 17 & 20.2 & 1.0902 & -0.0247 & 1.0146 & 0.3996 \\
\hline 18 & 21.3 & 1.0457 & -0.0374 & 0.9607 & 0.4147 \\
\hline 19 & 22.7 & 1.0750 & -0.0463 & 0.9739 & 0.4576 \\
\hline 20 & 23.9 & 0.9831 & -0.0568 & 0.8758 & 0.4502 \\
\hline 21 & 25.3 & 1.1523 & -0.0501 & 1.0204 & 0.5377 \\
\hline 22 & 26.4 & 1.1814 & -0.0456 & 1.0379 & 0.5661 \\
\hline 23 & 27.5 & 1.1152 & -0.0540 & 0.9643 & 0.5628 \\
\hline 24 & 28.7 & 1.1520 & -0.0521 & 0.9855 & 0.5989 \\
\hline 25 & 29.6 & 1.1393 & -0.0475 & 0.9672 & 0.6040 \\
\hline
\end{tabular}


Table 7.6: NREL S809 Airfoil Data from NASA-Ames Tests: 63.33\% Span

\begin{tabular}{|l|r|r|r|r|r|}
\hline $\begin{array}{l}\text { Wind } \\
\text { Speed } \\
m / s\end{array}$ & $\begin{array}{r}\text { Angle of } \\
\text { Attack }(\alpha) \\
\text { deg }\end{array}$ & $\begin{array}{r}\text { Dim.less } \\
\text { Normal } \\
\text { Coeff. }\left(C_{N}\right)\end{array}$ & $\begin{array}{r}\text { Dim.less } \\
\text { Tangential } \\
\text { Coeff. }\left(C_{T}\right)\end{array}$ & $\begin{array}{r}\text { Dim.less } \\
\text { Lift Coeff. } \\
\left(C_{L}\right)\end{array}$ & $\begin{array}{r}\text { Dim.less } \\
\text { Drag Coeff. } \\
\left(C_{D}\right)\end{array}$ \\
\hline 5 & 2.2 & 0.5479 & 0.0244 & 0.5484 & -0.0033 \\
\hline 6 & 4.1 & 0.7096 & 0.0573 & 0.7119 & -0.0064 \\
\hline 7 & 5.9 & 0.8656 & 0.0949 & 0.8708 & -0.0054 \\
\hline 8 & 7.8 & 0.9569 & 0.1386 & 0.9669 & -0.0075 \\
\hline 9 & 9.9 & 1.0804 & 0.1754 & 1.0945 & 0.0130 \\
\hline 10 & 11.8 & 1.1079 & 0.1790 & 1.1211 & 0.0513 \\
\hline 11 & 13.7 & 0.9257 & 0.0397 & 0.9088 & 0.1807 \\
\hline 12 & 15.5 & 1.0239 & 0.0159 & 0.9909 & 0.2583 \\
\hline 13 & 16.9 & 1.0926 & 0.0122 & 1.0490 & 0.3059 \\
\hline 14 & 18.6 & 1.2424 & 0.0070 & 1.1797 & 0.3896 \\
\hline 15 & 20.3 & 1.3169 & 0.0038 & 1.2364 & 0.4533 \\
\hline 16 & 22.2 & 1.3566 & -0.0034 & 1.2548 & 0.5157 \\
\hline 17 & 23.7 & 1.3006 & -0.0286 & 1.1794 & 0.5490 \\
\hline 18 & 25.0 & 1.3348 & -0.0171 & 1.2025 & 0.5796 \\
\hline 19 & 26.6 & 1.2452 & -0.0204 & 1.1043 & 0.5758 \\
\hline 20 & 27.8 & 1.2303 & -0.0356 & 1.0717 & 0.6053 \\
\hline 21 & 29.5 & 1.1861 & -0.0348 & 1.0152 & 0.6144 \\
\hline 22 & 30.8 & 1.1859 & -0.0393 & 0.9985 & 0.6410 \\
\hline 23 & 32.4 & 1.2114 & -0.0305 & 1.0065 & 0.6749 \\
\hline 24 & 33.8 & 1.2389 & -0.0317 & 1.0119 & 0.7155 \\
\hline 25 & 35.2 & 1.2520 & -0.0296 & 1.0060 & 0.7459 \\
\hline
\end{tabular}


Table 7.7: NREL S809 Airfoil Data from NASA-Ames Tests: $46.67 \%$ Span

\begin{tabular}{|l|r|r|r|r|r|}
\hline $\begin{array}{l}\text { Wind } \\
\text { Speed } \\
m / s\end{array}$ & $\begin{array}{r}\text { Angle of } \\
\text { Attack }(\alpha) \\
\text { deg }\end{array}$ & $\begin{array}{r}\text { Dim.less } \\
\text { Normal } \\
\text { Coeff. }\left(C_{N}\right)\end{array}$ & $\begin{array}{r}\text { Dim.less } \\
\text { Tangential } \\
\text { Coeff. }\left(C_{T}\right)\end{array}$ & $\begin{array}{r}\text { Dim.less } \\
\text { Lift Coeff. } \\
\left(C_{L}\right)\end{array}$ & $\begin{array}{r}\text { Dim.less } \\
\text { Drag Coeff. } \\
\left(C_{D}\right)\end{array}$ \\
\hline 5 & 5.0 & 0.5382 & 0.0276 & 0.5386 & 0.0194 \\
\hline 6 & 7.7 & 0.7513 & 0.0694 & 0.7538 & 0.0319 \\
\hline 7 & 10.4 & 0.9124 & 0.1177 & 0.9187 & 0.0489 \\
\hline 8 & 12.8 & 1.1681 & 0.1855 & 1.1802 & 0.0779 \\
\hline 9 & 15.5 & 1.2652 & 0.2243 & 1.2791 & 0.1220 \\
\hline 10 & 18.0 & 1.3460 & 0.0069 & 1.2823 & 0.4094 \\
\hline 11 & 20.4 & 1.4892 & 0.0300 & 1.4063 & 0.4910 \\
\hline 12 & 22.9 & 1.4273 & -0.0191 & 1.3074 & 0.5730 \\
\hline 13 & 24.8 & 1.3293 & -0.0412 & 1.1894 & 0.5950 \\
\hline 14 & 26.8 & 1.2439 & -0.0512 & 1.0872 & 0.6065 \\
\hline 15 & 28.8 & 1.2576 & -0.0509 & 1.0775 & 0.6505 \\
\hline 16 & 30.8 & 1.2750 & -0.0488 & 1.0702 & 0.6948 \\
\hline 17 & 32.7 & 1.3289 & -0.0454 & 1.0938 & 0.7561 \\
\hline 18 & 34.1 & 1.3859 & -0.0451 & 1.1223 & 0.8143 \\
\hline 19 & 36.0 & 1.4434 & -0.0422 & 1.1429 & 0.8825 \\
\hline 20 & 37.6 & 1.4912 & -0.0417 & 1.1560 & 0.9429 \\
\hline 21 & 39.2 & 1.5160 & -0.0383 & 1.1506 & 0.9878 \\
\hline 22 & 40.5 & 1.5432 & -0.0365 & 1.1498 & 1.0300 \\
\hline 23 & 42.1 & 1.5567 & -0.0356 & 1.1312 & 1.0701 \\
\hline 24 & 43.4 & 1.5716 & -0.0312 & 1.1204 & 1.1025 \\
\hline 25 & 44.6 & 1.5805 & -0.0296 & 1.1046 & 1.1308 \\
\hline
\end{tabular}


Table 7.8: NREL S809 Airfoil Data from NASA-Ames Tests: $30 \%$ Span

\begin{tabular}{|l|r|r|r|r|r|}
\hline $\begin{array}{l}\text { Wind } \\
\text { Speed } \\
\mathrm{m} / \mathrm{s}\end{array}$ & $\begin{array}{r}\text { Angle of } \\
\text { Attack }(\alpha) \\
\text { deg }\end{array}$ & $\begin{array}{r}\text { Dim.less } \\
\text { Normal } \\
\text { Coeff. }\left(C_{N}\right)\end{array}$ & $\begin{array}{r}\text { Dim.less } \\
\text { Tangential } \\
\text { Coeff. }\left(C_{T}\right)\end{array}$ & $\begin{array}{r}\text { Dim.less } \\
\text { Lift Coeff. } \\
\left(C_{L}\right)\end{array}$ & $\begin{array}{r}\text { Dim.less } \\
\text { Drag Coeff. } \\
\left(C_{D}\right)\end{array}$ \\
\hline 5 & 1.4 & 0.3651 & 0.0032 & 0.3651 & 0.0057 \\
\hline 6 & 4.7 & 0.5980 & 0.0422 & 0.5994 & 0.0069 \\
\hline 7 & 8.4 & 0.8048 & 0.0963 & 0.8102 & 0.0223 \\
\hline 8 & 12.1 & 1.0930 & 0.1816 & 1.1068 & 0.0515 \\
\hline 9 & 15.9 & 1.1980 & 0.2148 & 1.2110 & 0.1216 \\
\hline 10 & 19.2 & 1.2941 & 0.1767 & 1.2802 & 0.2587 \\
\hline 11 & 22.0 & 1.4932 & 0.1498 & 1.4406 & 0.4205 \\
\hline 12 & 24.8 & 1.7765 & 0.1397 & 1.6713 & 0.6183 \\
\hline 13 & 27.2 & 2.0447 & 0.1697 & 1.8962 & 0.7837 \\
\hline 14 & 29.6 & 2.4150 & 0.1232 & 2.1607 & 1.0857 \\
\hline 15 & 31.9 & 2.4481 & 0.0518 & 2.1057 & 1.2497 \\
\hline 16 & 33.9 & 2.4153 & 0.0185 & 2.0150 & 1.3318 \\
\hline 17 & 36.0 & 2.4058 & 0.0047 & 1.9491 & 1.4103 \\
\hline 18 & 37.1 & 2.3882 & -0.0018 & 1.9037 & 1.4420 \\
\hline 19 & 39.1 & 2.3696 & -0.0032 & 1.8369 & 1.4969 \\
\hline 20 & 40.5 & 2.3898 & -0.0013 & 1.8164 & 1.5530 \\
\hline 21 & 42.0 & 2.3624 & -0.0059 & 1.7517 & 1.5851 \\
\hline 22 & 43.2 & 2.3770 & -0.0023 & 1.7312 & 1.6288 \\
\hline 23 & 44.6 & 2.3545 & 0.0006 & 1.6769 & 1.6528 \\
\hline 24 & 45.8 & 2.3182 & -0.0028 & 1.6142 & 1.6639 \\
\hline 25 & 46.8 & 2.2919 & -0.0002 & 1.5688 & 1.6709 \\
\hline
\end{tabular}

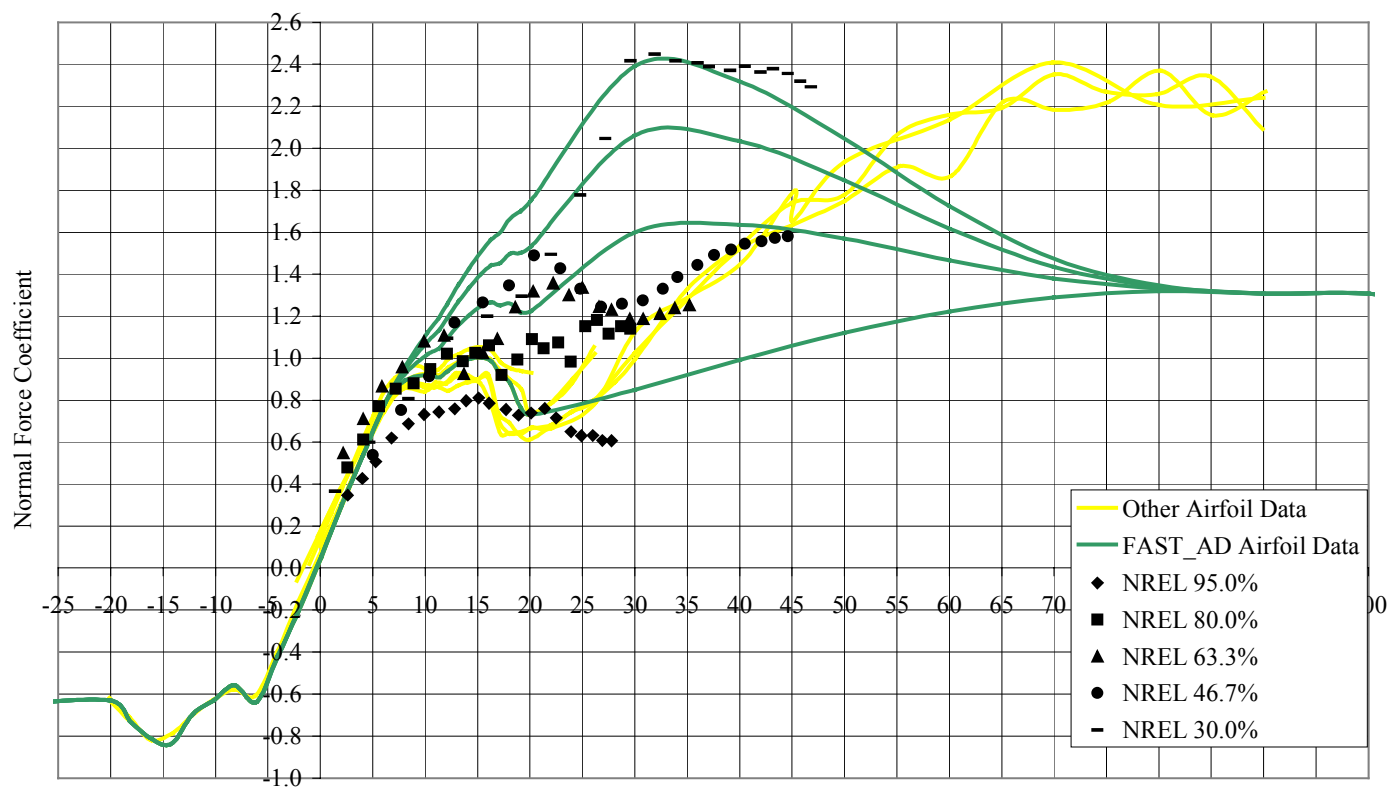

Angle of Attack [deg]

Figure 7.1: NREL S809 dimensionless normal force coefficient airfoil data 


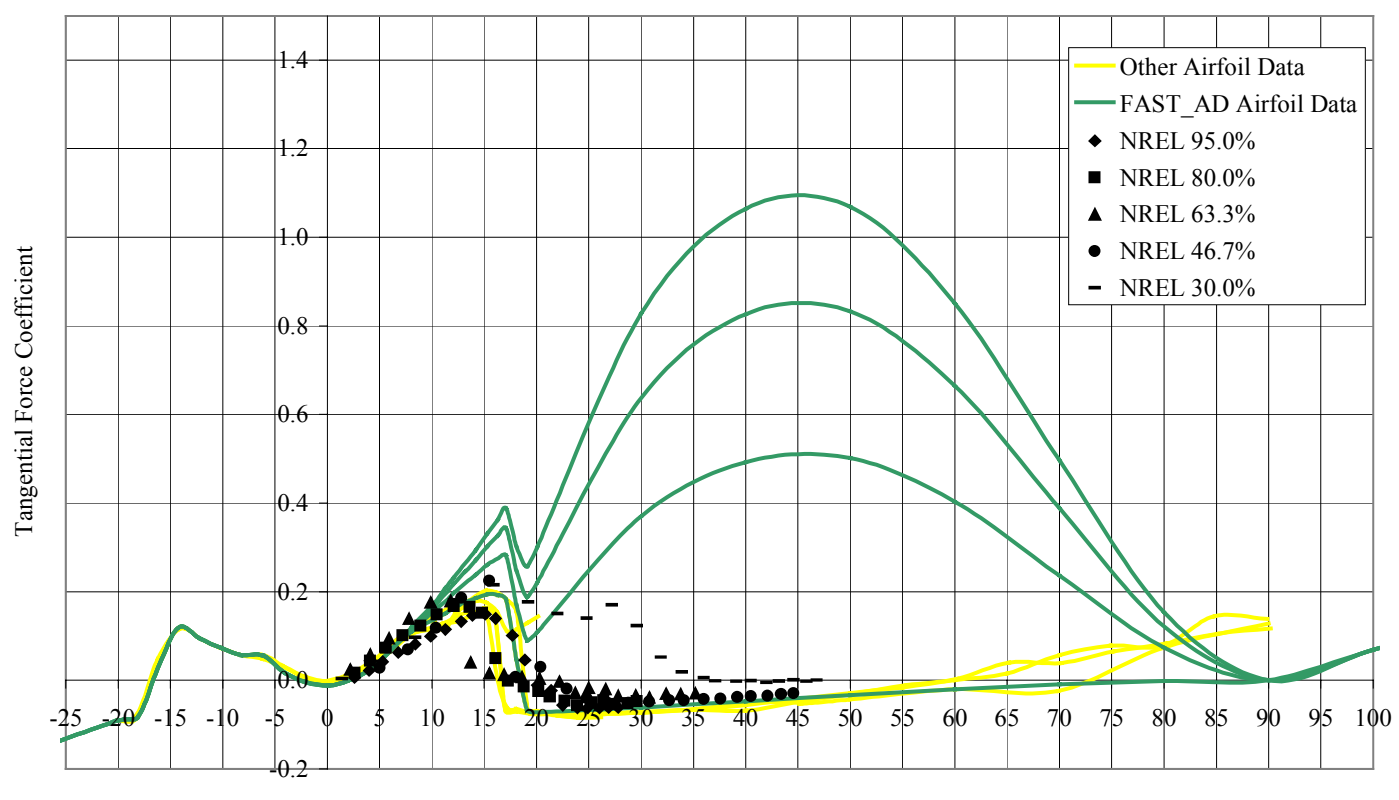

Angle of Attack [deg]

Figure 7.2: NREL S809 dimensionless tangential force coefficient airfoil data

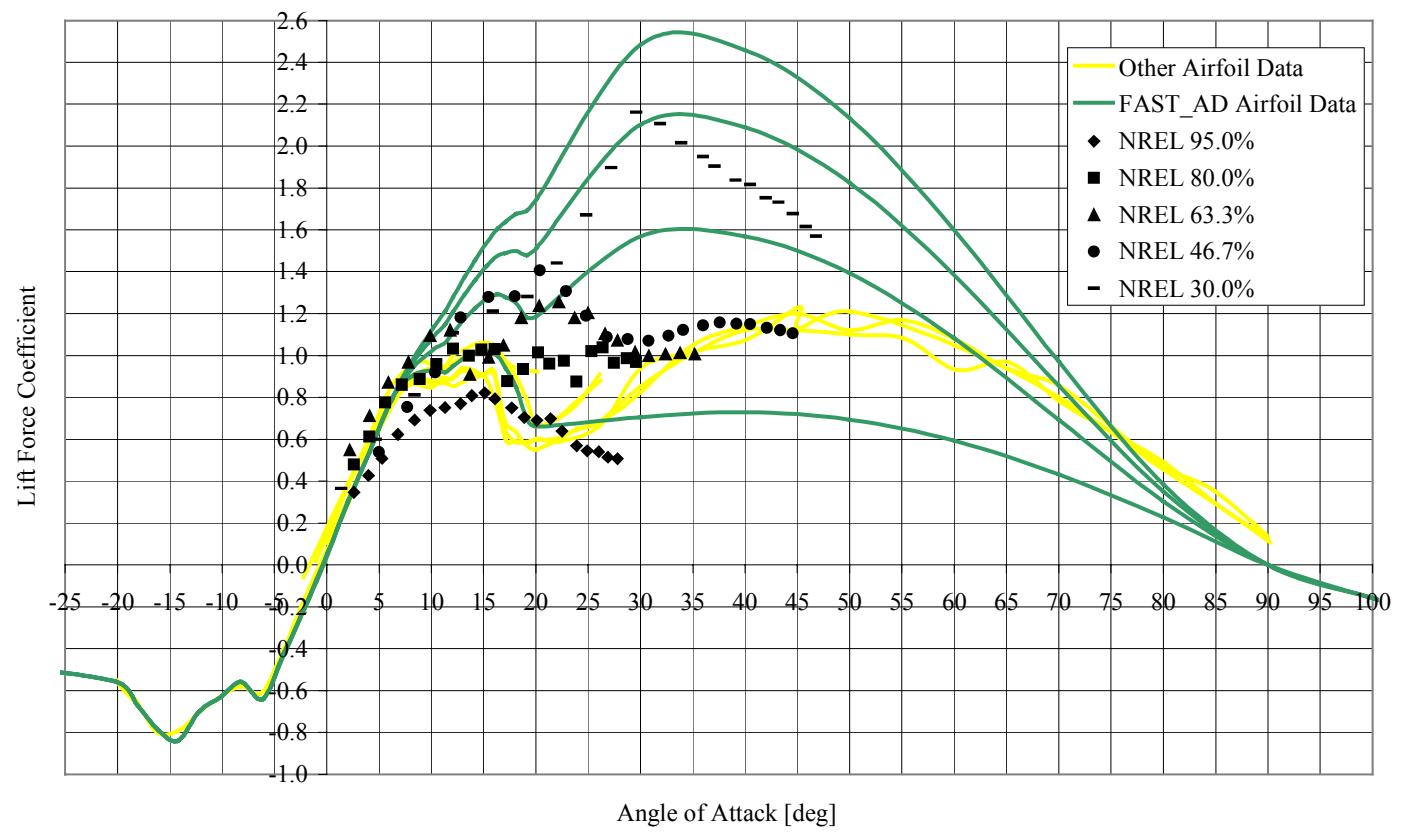

Figure 7.3: NREL S809 dimensionless lift force coefficient airfoil data 


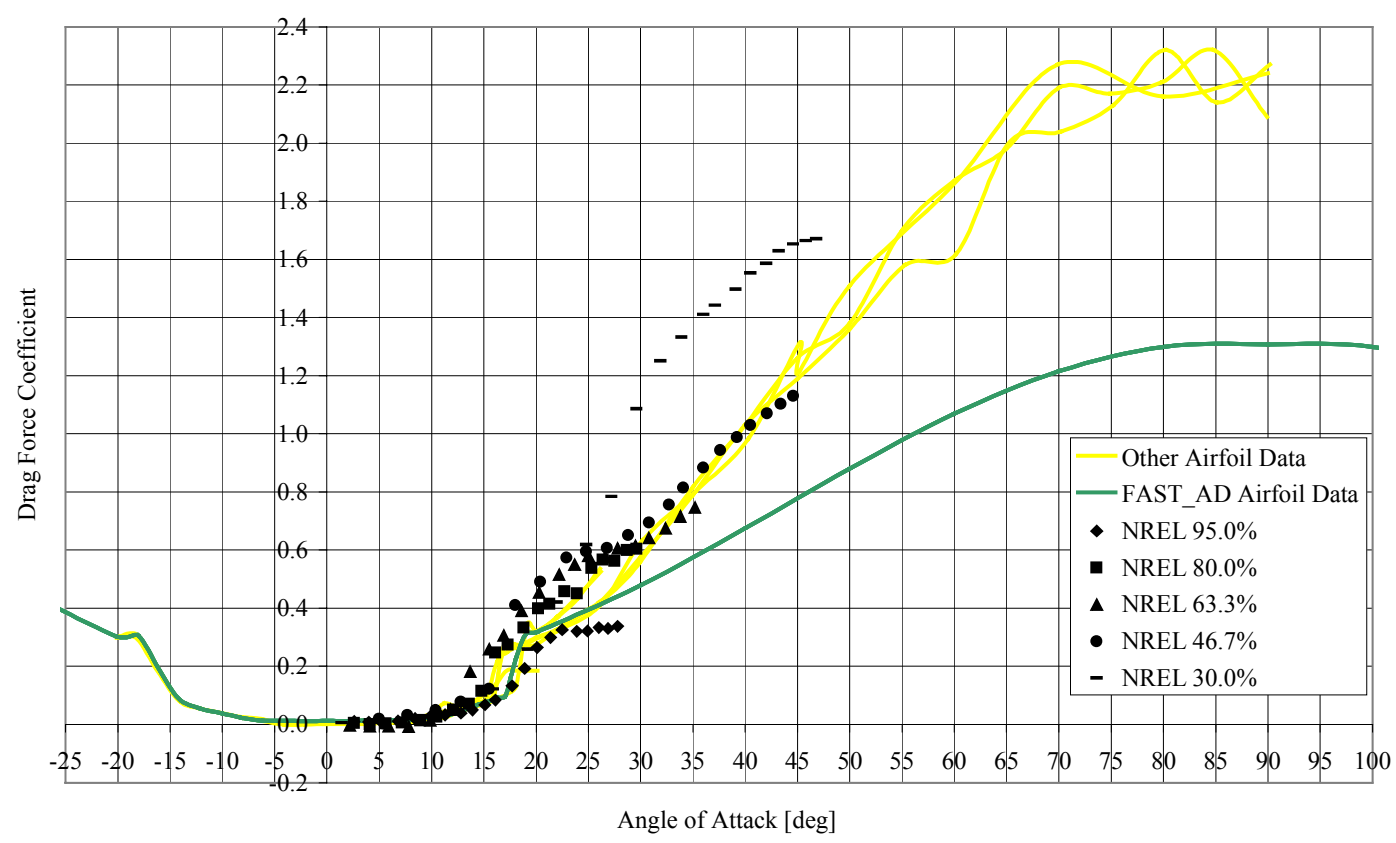

Figure 7.4: NREL S809 dimensionless drag force coefficient airfoil data

In Figs. 7.1-7.4, the curves labeled "FAST_AD Airfoil Data" illustrate some of the different dimensionless force coefficient airfoil data used by FAST_AD at the various blade elements for the Blind Comparison work. The curves labeled "Other Airfoil Data" illustrate the unmodified airfoil data measured in the static, two-dimensional environment of the CSU, OSU, and DUT wind tunnel tests. The dissimilarities between the unmodified and extrapolated two-dimensional airfoil data and the airfoil data measured in the three-dimensional environment of the NASAAmes wind tunnel tests are clearly visible. Therefore, three-dimensional flow effects, such as spanwise pressure gradients, spanwise flow, flow circulation, and vorticity, prohibit the use of unmodified two-dimensional airfoil data at all span stations. This is manifest even in the supposedly attached flow, linear, pre-stall regions! The "NREL **.*\%" data are surprisingly coherent, considering each data point was measured in a separate wind tunnel test. This further underscores the accuracy and repeatability of NASA-Ames wind tunnel tests (see section 5.2).

In the sensitivity study titled "NASAAmesAirfoilData," the five airfoil data tables that reflect the measurements made in the three-dimensional environment of the NASA-Ames wind tunnel tests (given in Tables 7.4-7.8) are used for the blade element airfoil data in FAST_AD. This study demonstrates how accurately the model can pinpoint the correct force coefficient-angle of attack pair at each span station when very accurate aerodynamic properties of the airfoil at each span station are given. Since the BEM curves [Eq. (2.73)] are not dependent on airfoil data, Figs. 6.23 and 6.24 reveal that the exact force coefficient-angle of attack pairs will not be predicted correctly at the 7 and $10 \mathrm{~m} / \mathrm{s}$ wind speeds even before the simulations are run. To be predicted correctly, the BEM curves in these figures would have to pass through their respective "NREL" data points, which they do not, in almost every instance. These figures indicate that the angle of attack will be mispredicted in nearly every situation. Thus, stated another way, the "NASAAmesAirfoilData" study demonstrates the sensitivity of the model to mispredictions in angle of attack. As indicated at the end of Chapter 6, this is especially critical in the attached flow and stall regimes. 
For use in FAST_AD, the three-dimensional environment, NASA-Ames airfoil data tables must be extended across the entire $360^{\circ}$ range of potential angles of attack. To obtain the absent deep stall force coefficient angle of attack relationships, the utility program titled "FoilCheck," which employs the Viterna-Corrigan equations of post stall, is used. As in the Blind Comparison work, an aspect ratio of 11 is used during the application of these equations. Also, since NASA-Ames airfoil data tables are available only at five blade span stations, the data tables are correlated to the most well-suited node. As such, the airfoil data table reflecting the conditions seen at $95 \%$ span is coupled to nodes 19-21. Similarly, the airfoil data table reflecting the conditions seen at $80 \%$ span is coupled to nodes $15-18$, the airfoil data table for $63.33 \%$ span is coupled to nodes $12-14$, the airfoil data table for $46.67 \%$ span is coupled to nodes $8-11$, and the airfoil data table for $30 \%$ span is coupled to nodes $2-7$. The dimensionless coefficient airfoil data for a smooth circular cylinder are again applied at node 1, the innermost node.

As seen in the next section, mispredictions in angle of attack for the "NASAAmesAirfoilData" study do have an impact on the accuracy of predicted aerodynamic loads when compared to experimentally measured loads (see Figs. 7.7-7.16). Predictions of low-speed shaft torque, root flap bending, and root edge bending moments are also inaccurate for this study (see Figs. 7.227.24). This raises the question of whether these moments would be accurately predicted if the aerodynamic loads were predicted accurately at each span station and wind speed. In other words, do the discrepancies between the "NASAAmesAirfoilData" study aerodynamic load predictions and the loads measured during the NASA-Ames wind tunnel tests in Figs. 7.7-7.16 account for the discrepancies in the predicted and measured moments seen in Figs. 7.22-7.24? This is a refinement of question (3) in the introduction to this chapter.

This question is addressed in the sensitivity study entitled "NASAAmesAlpha." To answer the question, the angle of attack misprediction must be eliminated since elimination of aerodynamic load discrepancies is a direct outcome of this action. The inaccuracy in angle of attack predictions for the "NASAAmesAirfoilData" study is characterized in Figs. 7.5 and 7.6. The curves given in Fig. 7.6 represent the difference between the curves given in Fig. 7.5 for each discrete wind speed test case. The curve labeled "Average" represents the mean of these curves. 


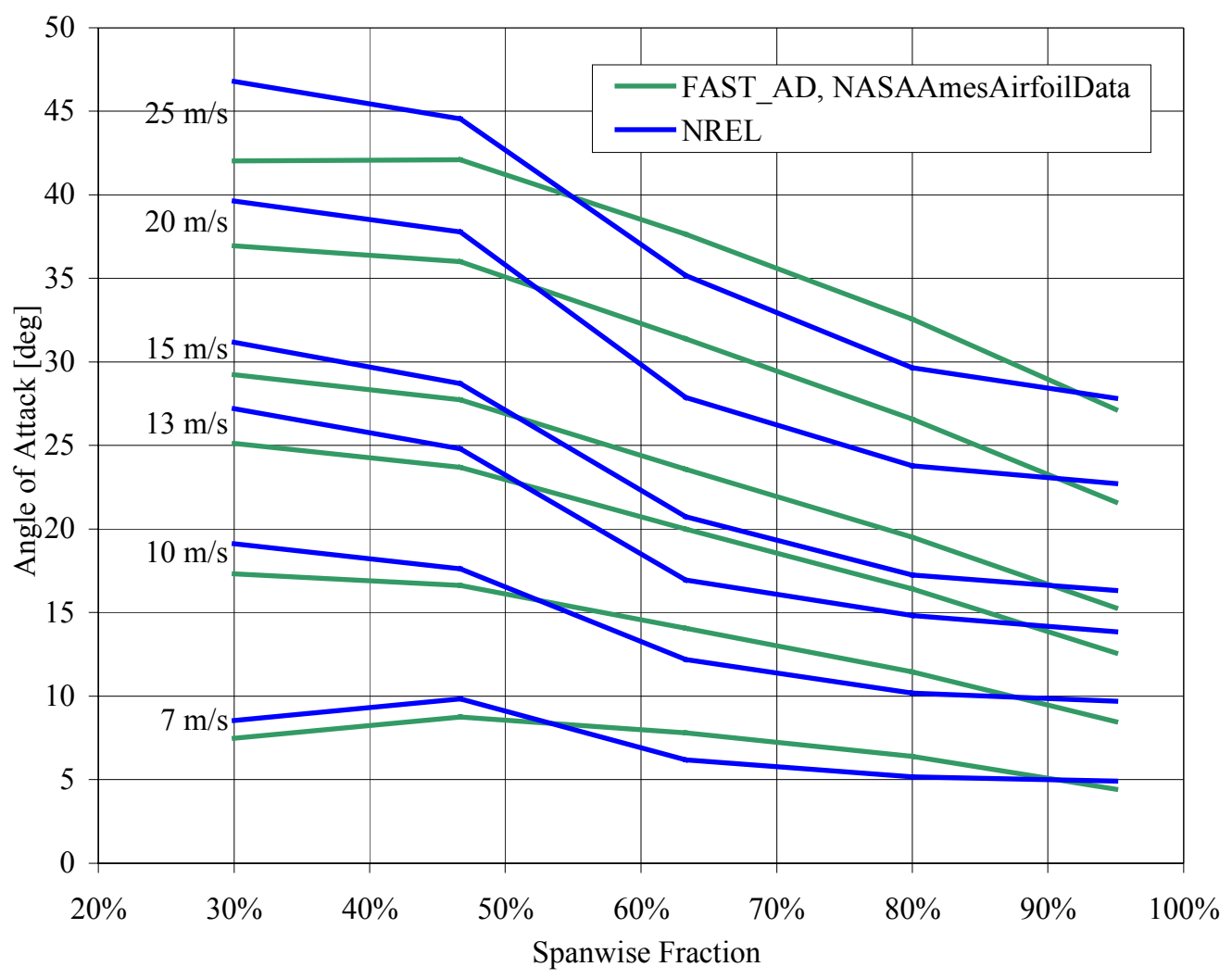

Figure 7.5: “NASAAmesAirfoilData” study angle of attack predictions

The curves in Fig. 7.6 clearly illustrate that the angle of attack is consistently underpredicted inboard, overpredicted midboard, and slightly underpredicted outboard at every wind speed. In the "NASAAmesAlpha" study, the aerodynamic twist distribution of both blades is modified to counteract these mispredictions in angle of attack by decreasing the local aerodynamic twist inboard, increasing it midboard, and decreasing it again outboard. Though slightly arbitrary, the magnitudes of these adjustments are set equal to the average angle of attack mispredictions shown in Fig. 7.6. The average differences are employed instead of the exact differences for each wind speed test case for a number of reasons:

(1) Modifying the aerodynamic twist distribution does not completely eliminate angle of attack prediction inaccuracies.

(2) The angle of attack probes are not precise and the measurements have to be upwash corrected, a process that too is not precise (see section 5.1). The end result is that the angle of attack distributions measured during the NASA-Ames wind tunnel tests are not entirely accurate.

(3) Manual effort is reduced. 


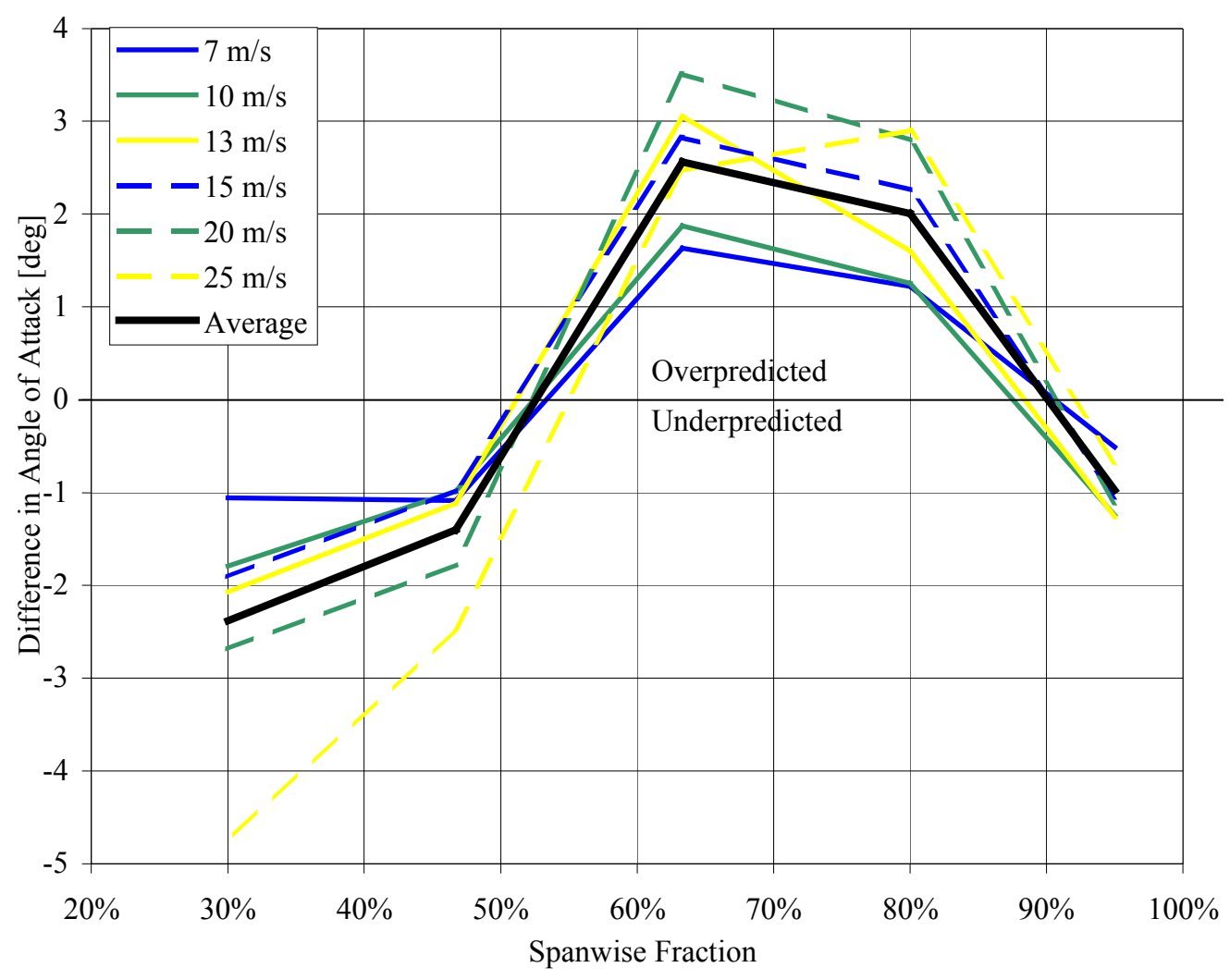

Figure 7.6: "NASAAmesAirfoilData" study angle of attack inaccuracies

Adjusted local twist angles for the 21 blade nodes are listed in Table 7.9. The structural pretwist distribution is altere accordingly. The same airfoil data used in the "NASAAmesAirfoilData" study are used for the "NASAAmesAlpha" study. 
Table 7.9: Nodal Twist Properties for Sensitivity Study "NASAAmesAlpha”

\begin{tabular}{|c|c|c|c|c|c|c|c|}
\hline \multirow[b]{3}{*}{$\begin{array}{l}\text { Node } \\
\text { Number }\end{array}$} & \multicolumn{4}{|c|}{ Node Location } & \multirow{2}{*}{\multicolumn{3}{|c|}{$\begin{array}{l}\text { Local Twist }\left(\theta_{T}\right) \\
\quad d e g\end{array}$}} \\
\hline & \multirow{2}{*}{$\begin{array}{l}\text { Distance } \\
\text { from Rotor } \\
\text { Center } \\
m\end{array}$} & \multirow{2}{*}{$\begin{array}{l}\text { Spanwise } \\
\text { Fraction } \\
(/ 5.029 \mathrm{~m})\end{array}$} & \multirow{2}{*}{\begin{tabular}{|l} 
Distance \\
Along Blade \\
From Root $(r)$ \\
$m$
\end{tabular}} & \multirow{2}{*}{$\begin{array}{l}\text { Radial } \\
\text { Fraction } \\
(r / 4.597 \mathrm{~m})\end{array}$} & & & \\
\hline & & & & & \begin{tabular}{r|} 
Original \\
Values \\
\end{tabular} & Alteration & $\begin{array}{r}\text { Adjusted } \\
\text { Values } \\
\end{array}$ \\
\hline 1 & 0.432 & 0.086 & 0.000 & 0.000 & 0.00 & 0.00 & 0.00 \\
\hline 2 & 0.662 & 0.132 & 0.230 & 0.050 & 0.00 & 0.00 & 0.00 \\
\hline 3 & 0.892 & 0.177 & 0.460 & 0.100 & 3.66 & -1.30 & 2.36 \\
\hline 4 & 1.122 & 0.223 & 0.690 & 0.150 & 12.78 & -2.35 & 10.43 \\
\hline 5 & 1.351 & 0.269 & 0.919 & 0.200 & 17.89 & -2.37 & 15.52 \\
\hline 6 & 1.581 & 0.314 & 1.149 & 0.250 & 13.33 & -2.30 & 11.03 \\
\hline 7 & 1.811 & 0.360 & 1.379 & 0.300 & 10.19 & -2.02 & 8.17 \\
\hline 8 & 2.041 & 0.406 & 1.609 & 0.350 & 7.24 & -1.72 & 5.52 \\
\hline 9 & 2.271 & 0.452 & 1.839 & 0.400 & 5.52 & -1.17 & 4.35 \\
\hline 10 & 2.501 & 0.497 & 2.069 & 0.450 & 3.81 & -0.63 & 3.18 \\
\hline 11 & 2.731 & 0.543 & 2.299 & 0.500 & 2.81 & 0.42 & 3.23 \\
\hline 12 & 2.960 & 0.589 & 2.528 & 0.550 & 1.86 & 1.50 & 3.36 \\
\hline 13 & 3.190 & 0.634 & 2.758 & 0.600 & 1.21 & 1.97 & 3.18 \\
\hline 14 & 3.420 & 0.680 & 2.988 & 0.650 & 0.66 & 2.23 & 2.89 \\
\hline 15 & 3.650 & 0.726 & 3.218 & 0.700 & 0.21 & 2.26 & 2.47 \\
\hline 16 & 3.880 & 0.771 & 3.448 & 0.750 & -0.15 & 2.10 & 1.95 \\
\hline 17 & 4.110 & 0.817 & 3.678 & 0.800 & \begin{tabular}{|c|}
-0.51 \\
\end{tabular} & 1.67 & 1.16 \\
\hline 18 & 4.339 & 0.863 & 3.907 & 0.850 & -0.84 & 0.76 & -0.08 \\
\hline 19 & 4.569 & 0.909 & 4.137 & 0.900 & -1.17 & -0.06 & -1.23 \\
\hline 20 & 4.799 & 0.954 & 4.367 & 0.950 & -1.49 & -0.52 & -2.01 \\
\hline 21 & 5.029 & 1.000 & 4.597 & 1.000 & -1.82 & -0.97 & -2.79 \\
\hline
\end{tabular}

\subsection{Sensitivity Study Results and Discussion}

Average dimensionless normal force coefficients at each pressure tap grouping span station predicted by FAST_AD for each sensitivity study are plotted against wind speed on the abscissa in Figs. 7.7-7.11. Corresponding tangential force coefficients are given in Figs. 7.12-7.16. Local dynamic pressures are given in Figs. 7.17-7.21. Plots of low-speed shaft torque, root flap bending moments, and root edge bending moments are given in Figs. 7.22-7.24. The curves labeled "FAST_AD" represent the data from the simulations performed for the Blind Comparison work, the results of which are described in Chapter 6. Data from the other Blind Comparison participants were omitted from these plots to avoid clutter and confusion. The curves labeled "NREL" represent the data measured during the NASA-Ames wind tunnel tests. 


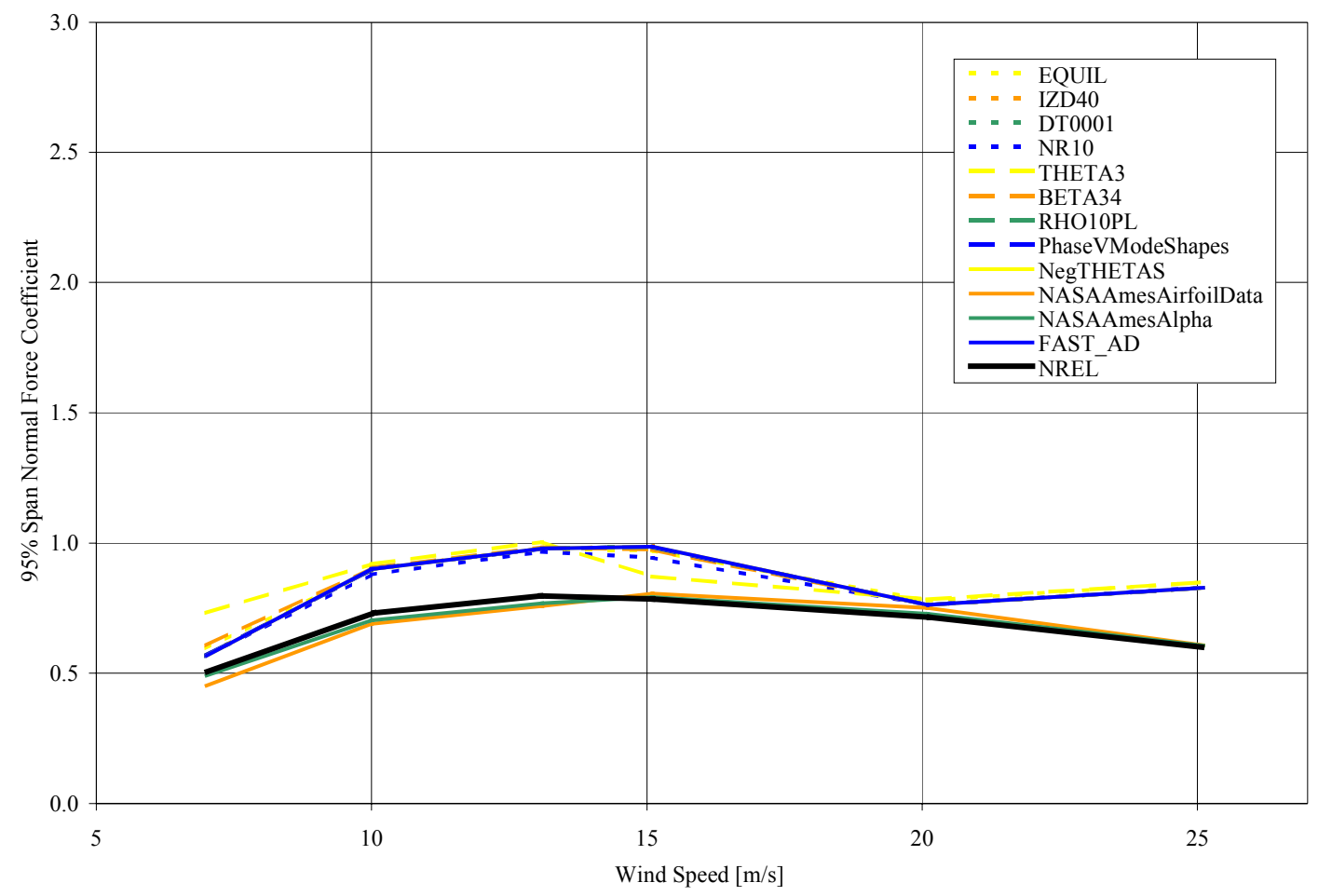

Figure 7.7: 95\% span normal force coefficient, upwind, zero yaw

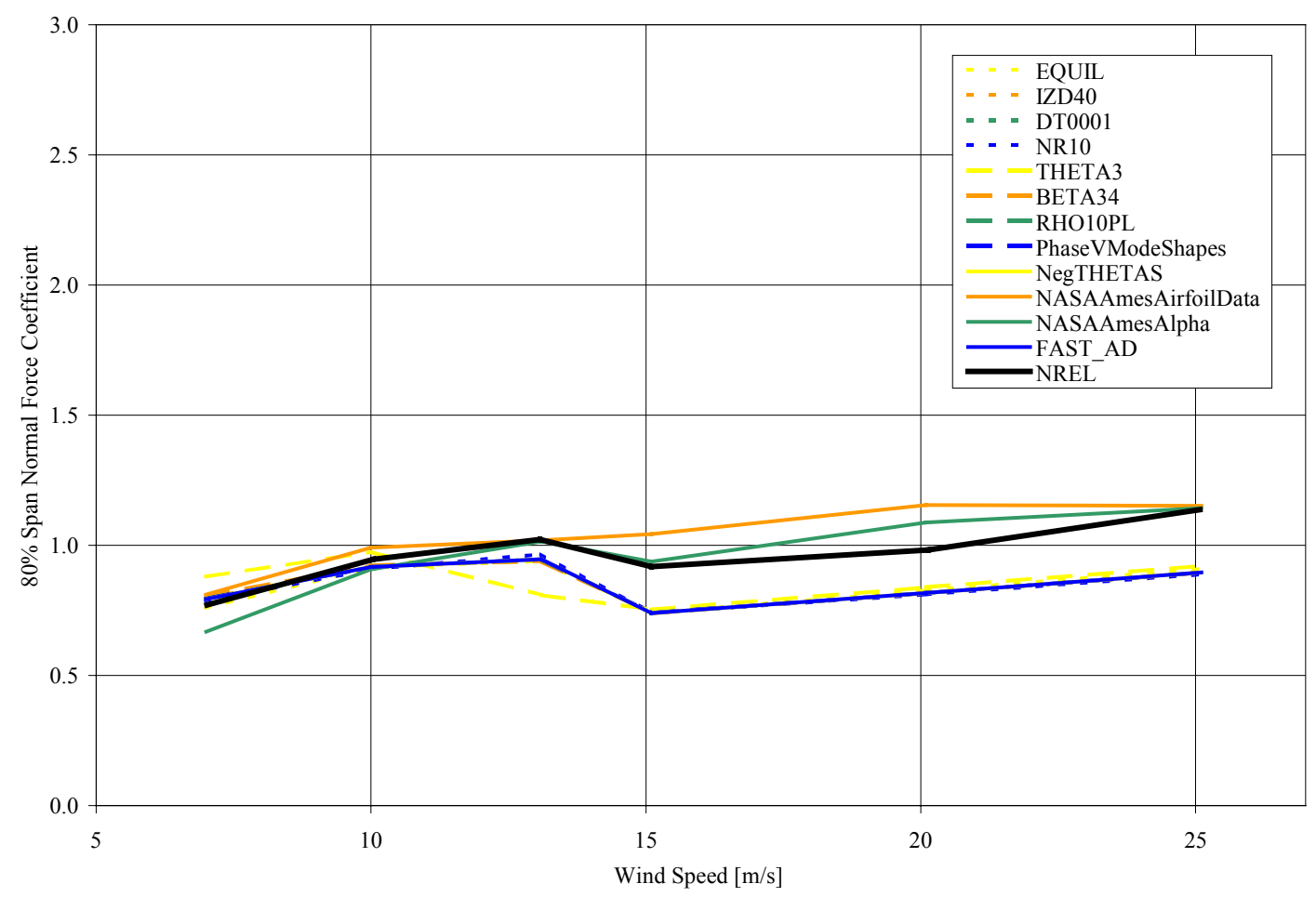

Figure 7.8: $80 \%$ span normal force coefficient, upwind, zero yaw 


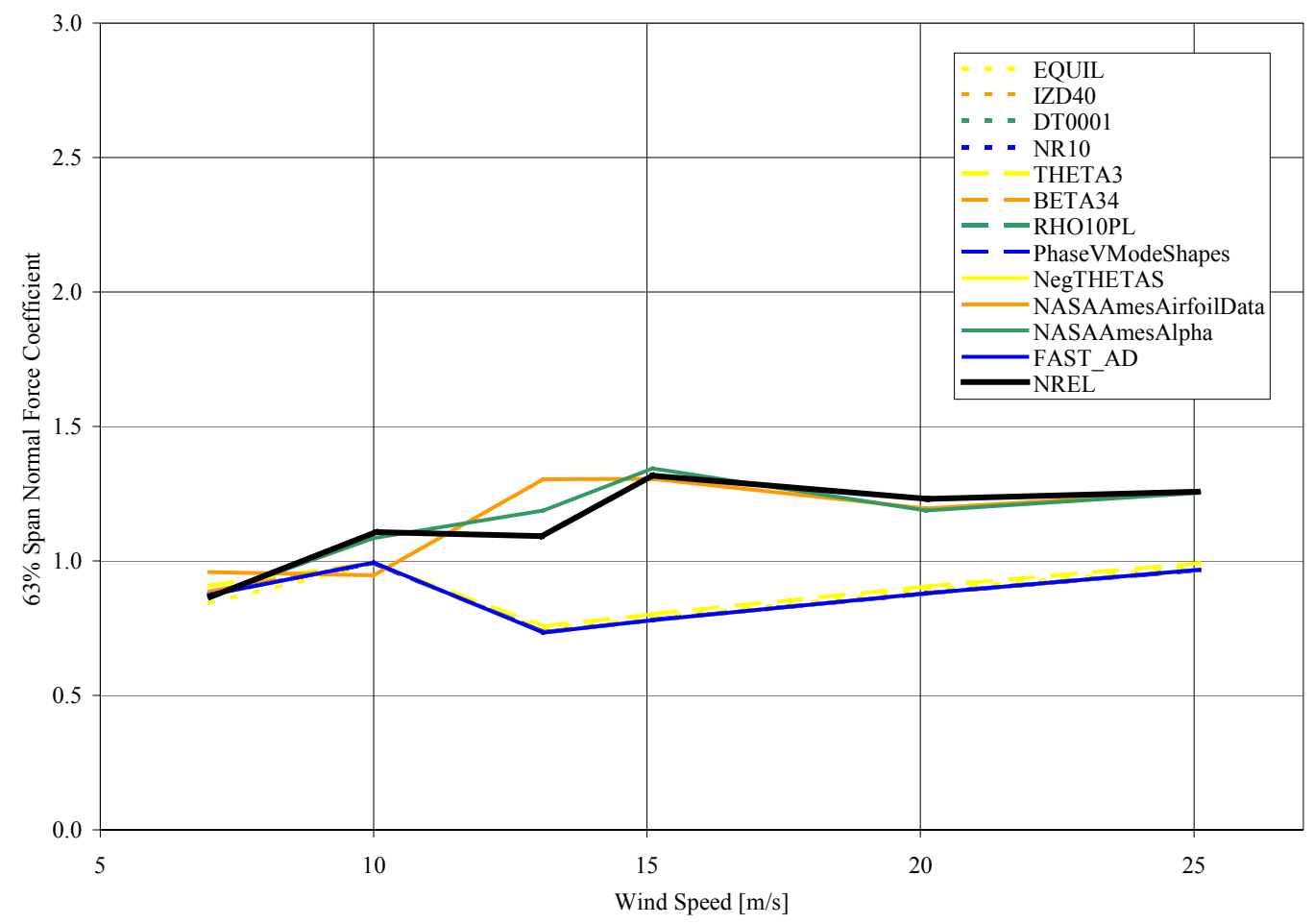

Figure 7.9: 63\% span normal force coefficient, upwind, zero yaw

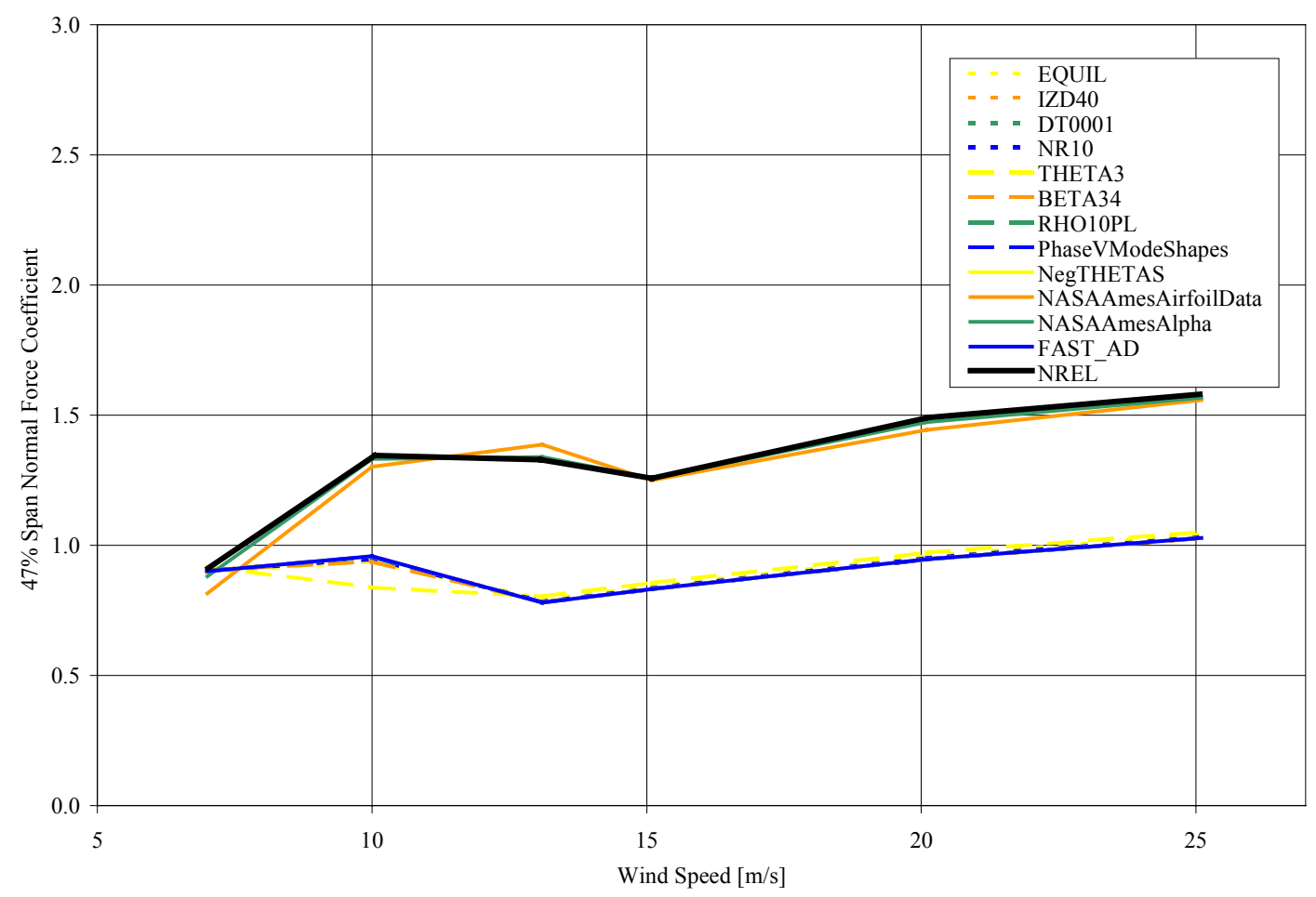

Figure 7.10: $47 \%$ span normal force coefficient, upwind, zero yaw 


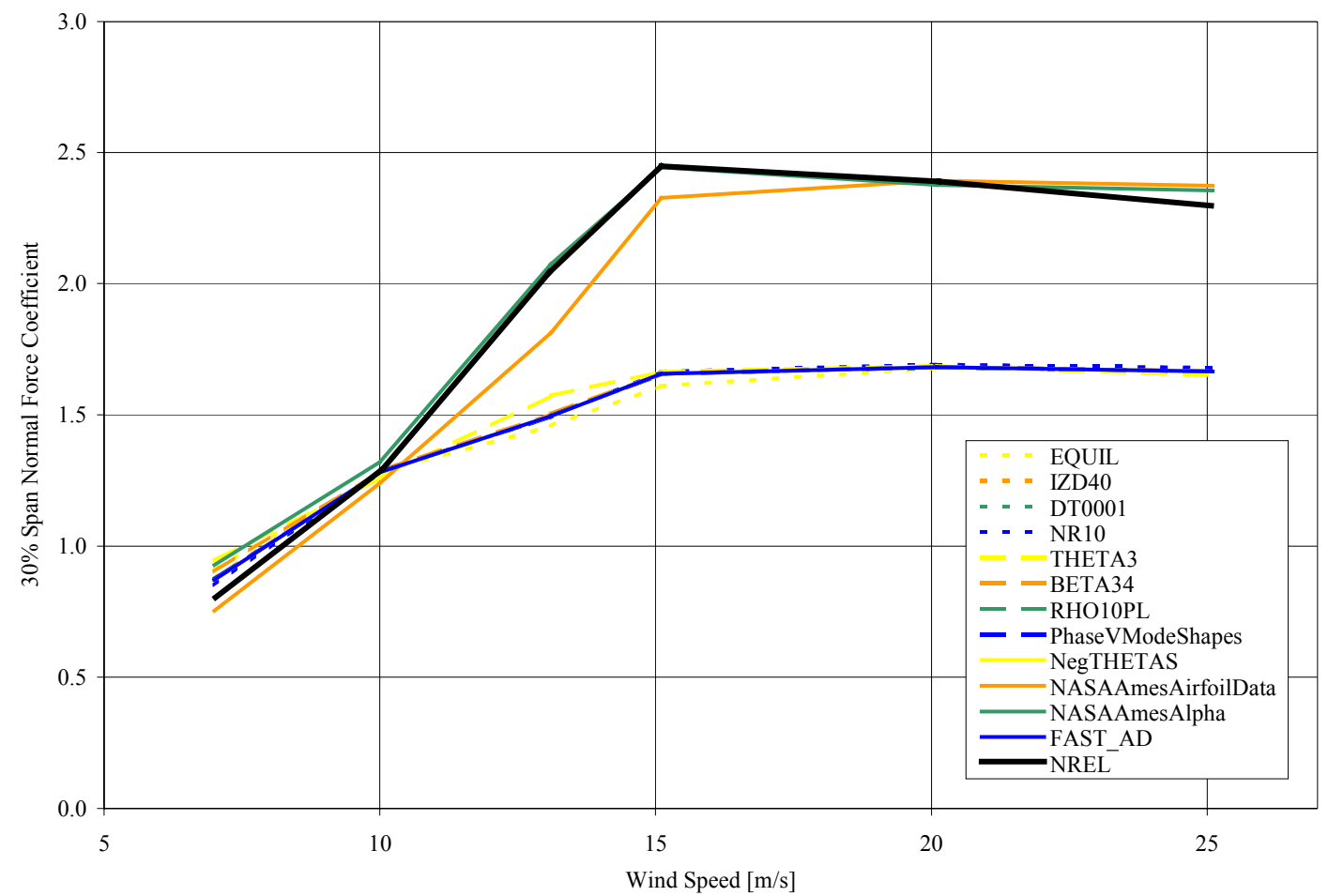

Figure 7.11: $30 \%$ span normal force coefficient, upwind, zero yaw

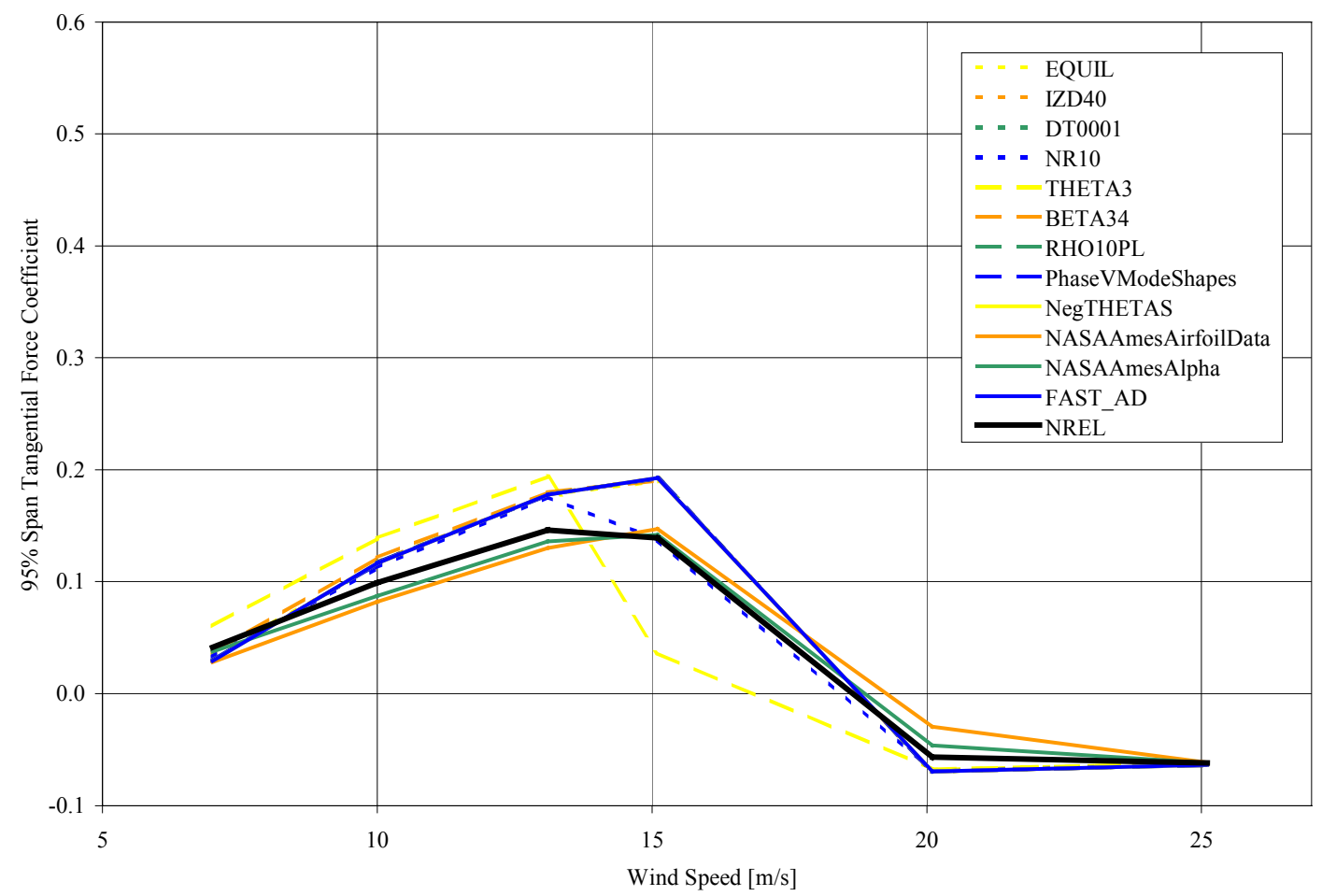

Figure 7.12: $95 \%$ span tangential force coefficient, upwind, zero yaw 


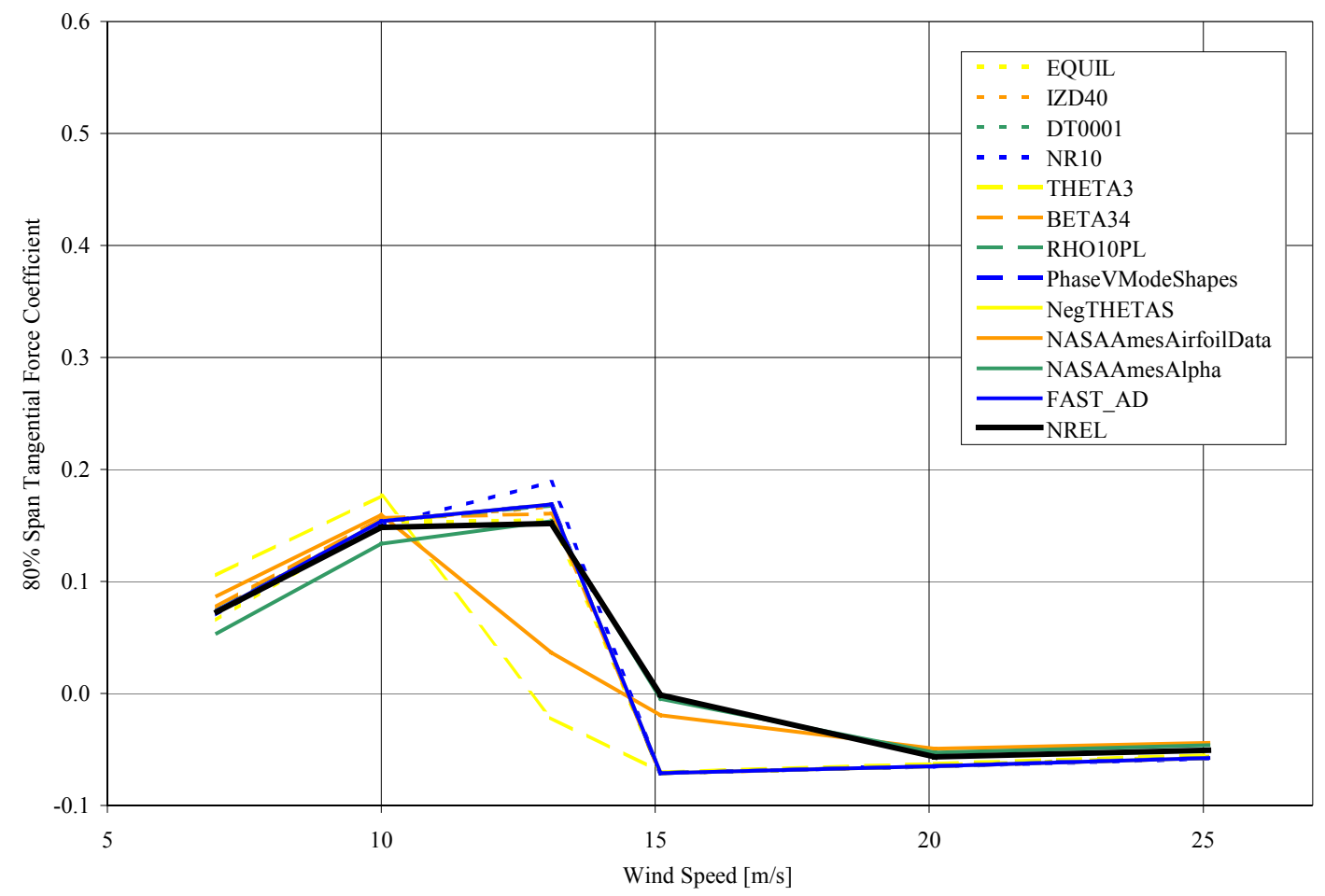

Figure $7.13: 80 \%$ span tangential force coefficient, upwind, zero yaw

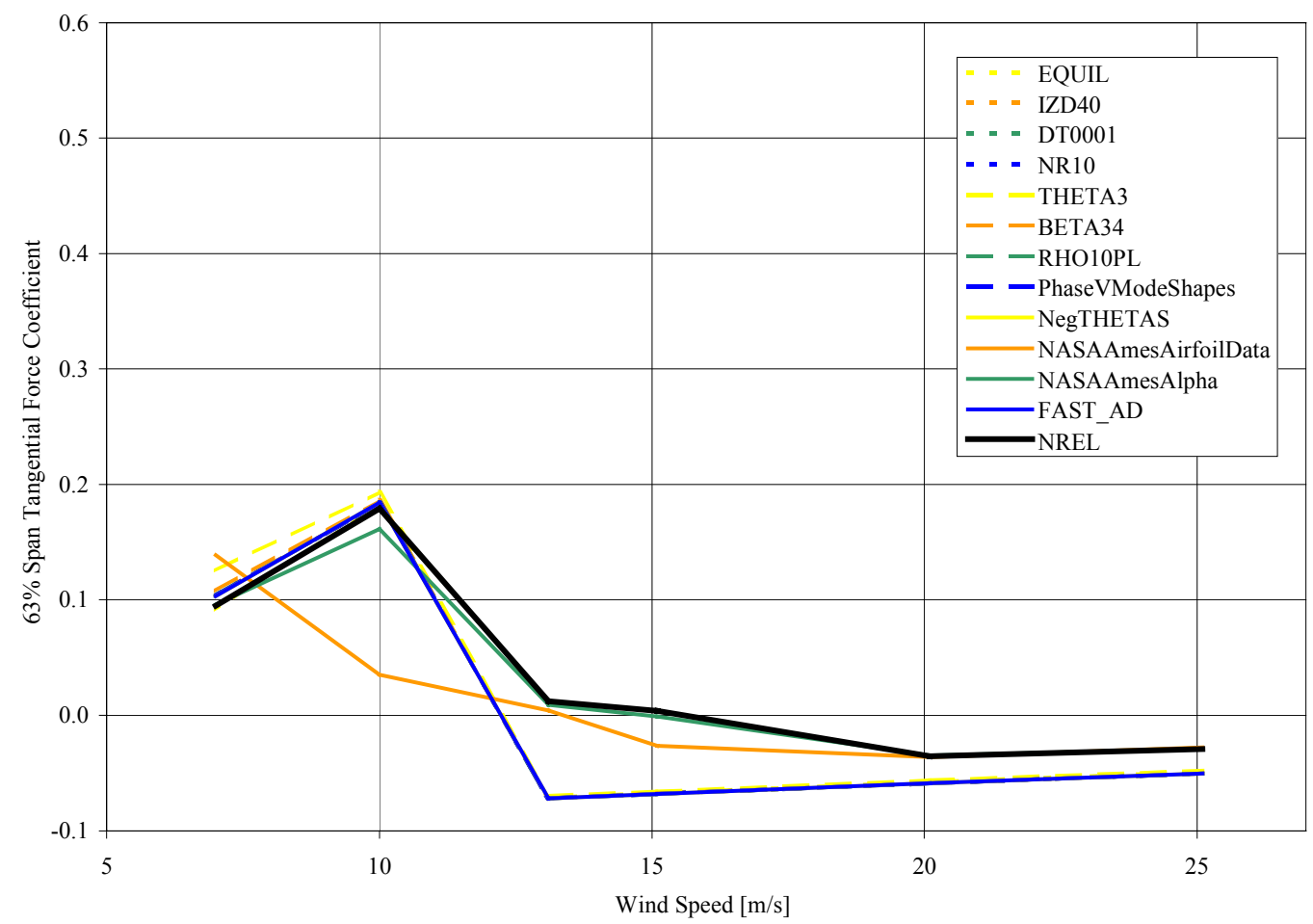

Figure 7.14: $63 \%$ span tangential force coefficient, upwind, zero yaw 


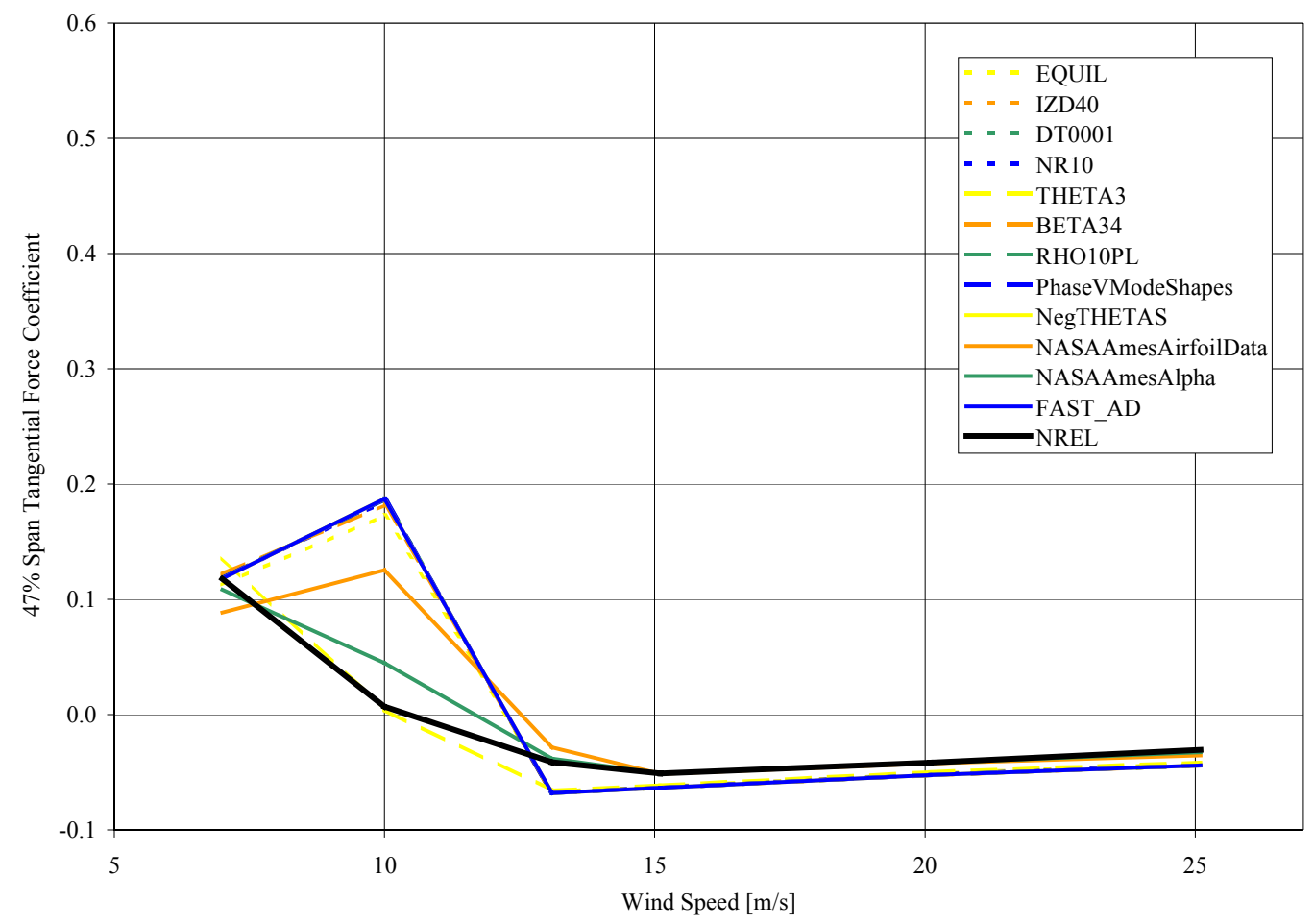

Figure $7.15: 47 \%$ span tangential force coefficient, upwind, zero yaw

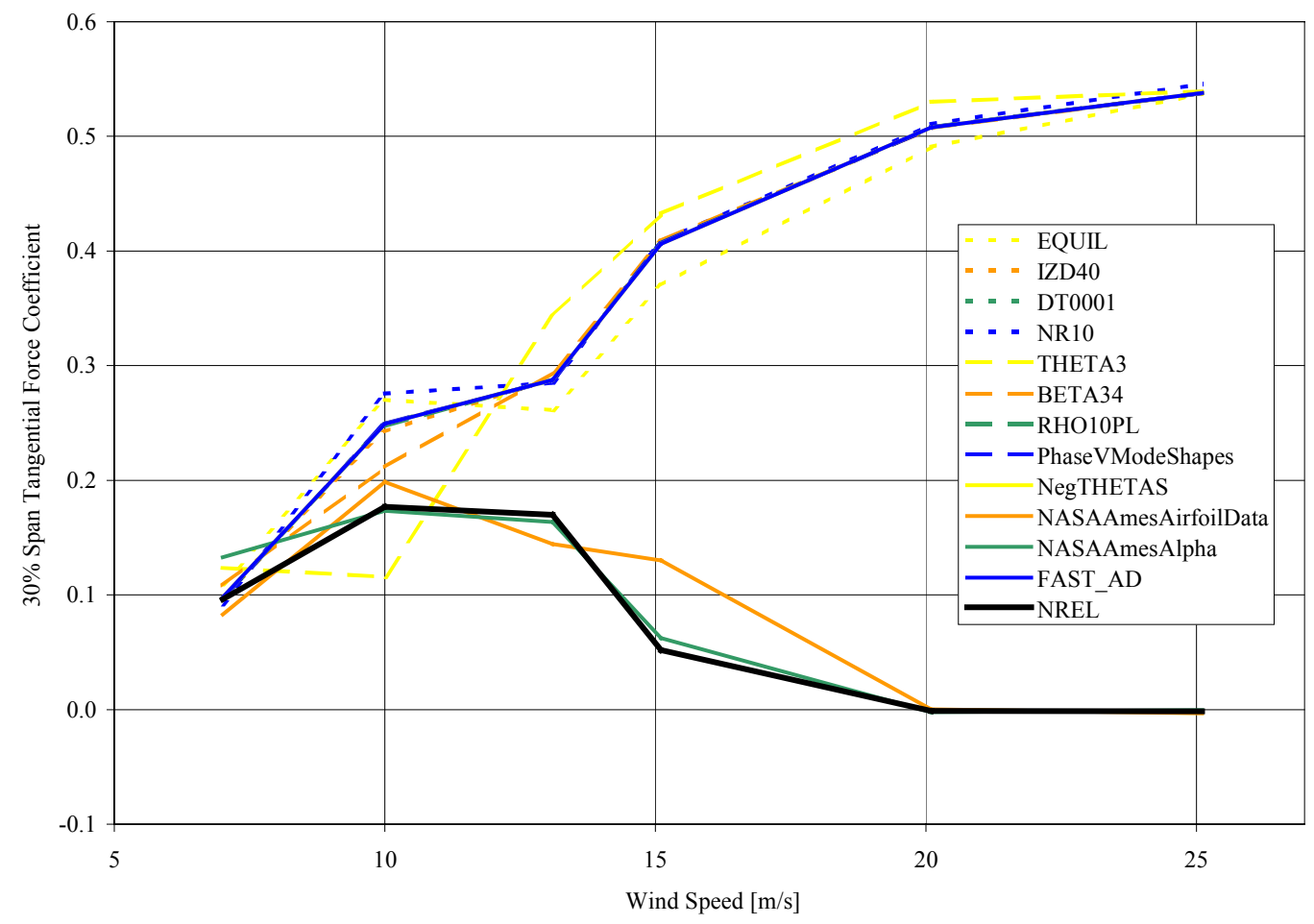

Figure 7.16 : $30 \%$ span tangential force coefficient, upwind, zero yaw 


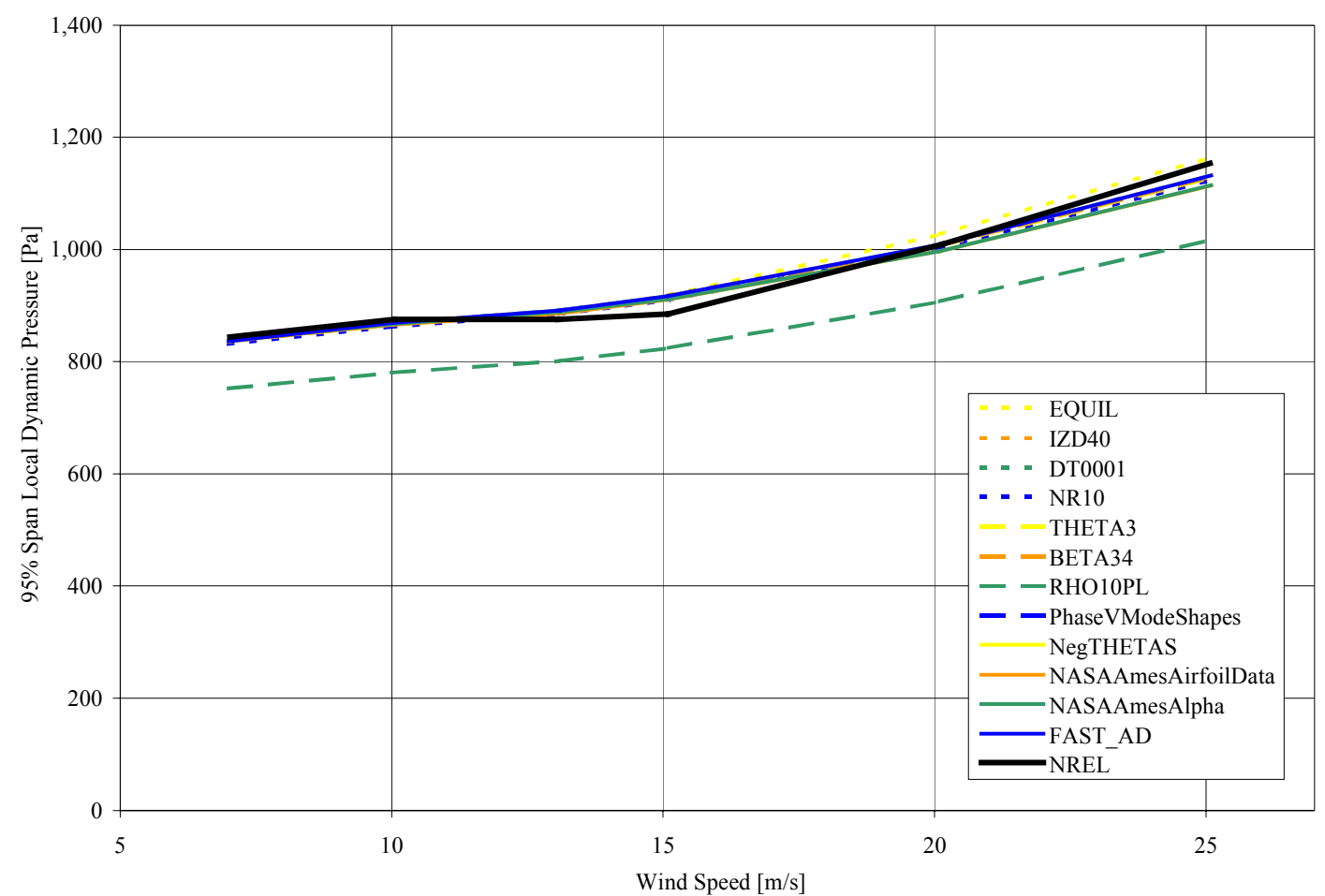

Figure 7.17: 95\% span local dynamic pressure, upwind, zero yaw

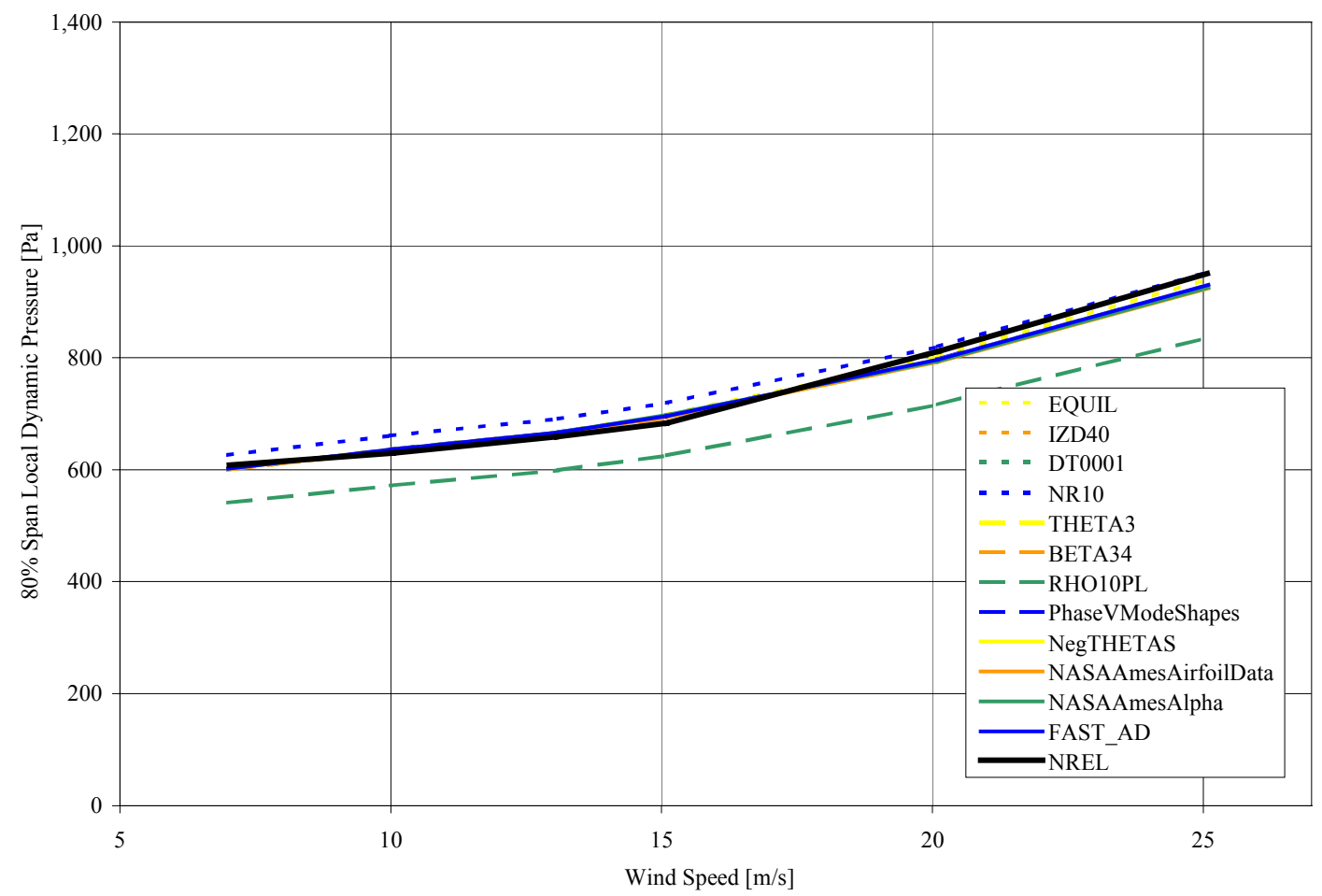

Figure 7.18: $80 \%$ span local dynamic pressure, upwind, zero yaw 


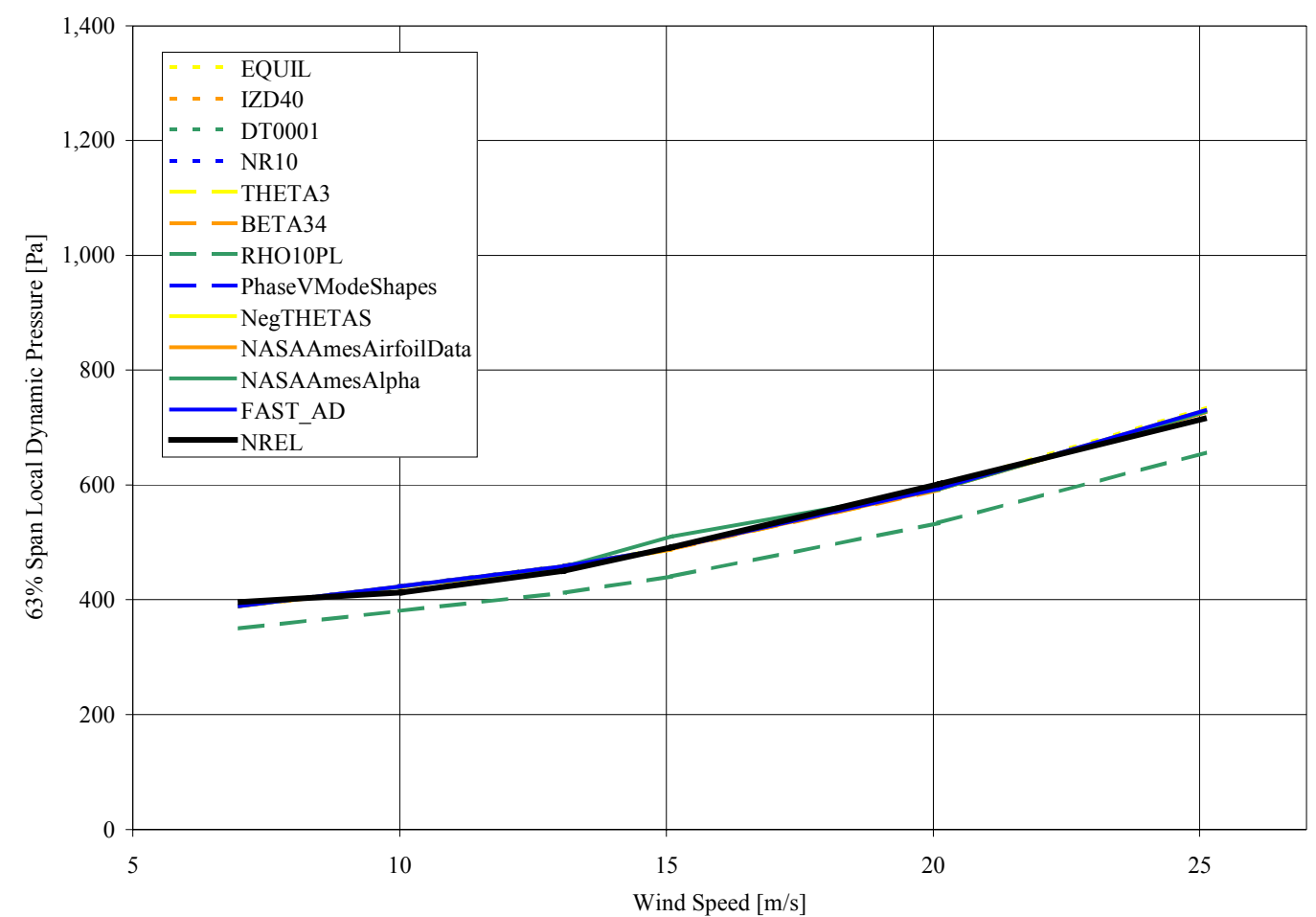

Figure 7.19: 63\% span local dynamic pressure, upwind, zero yaw

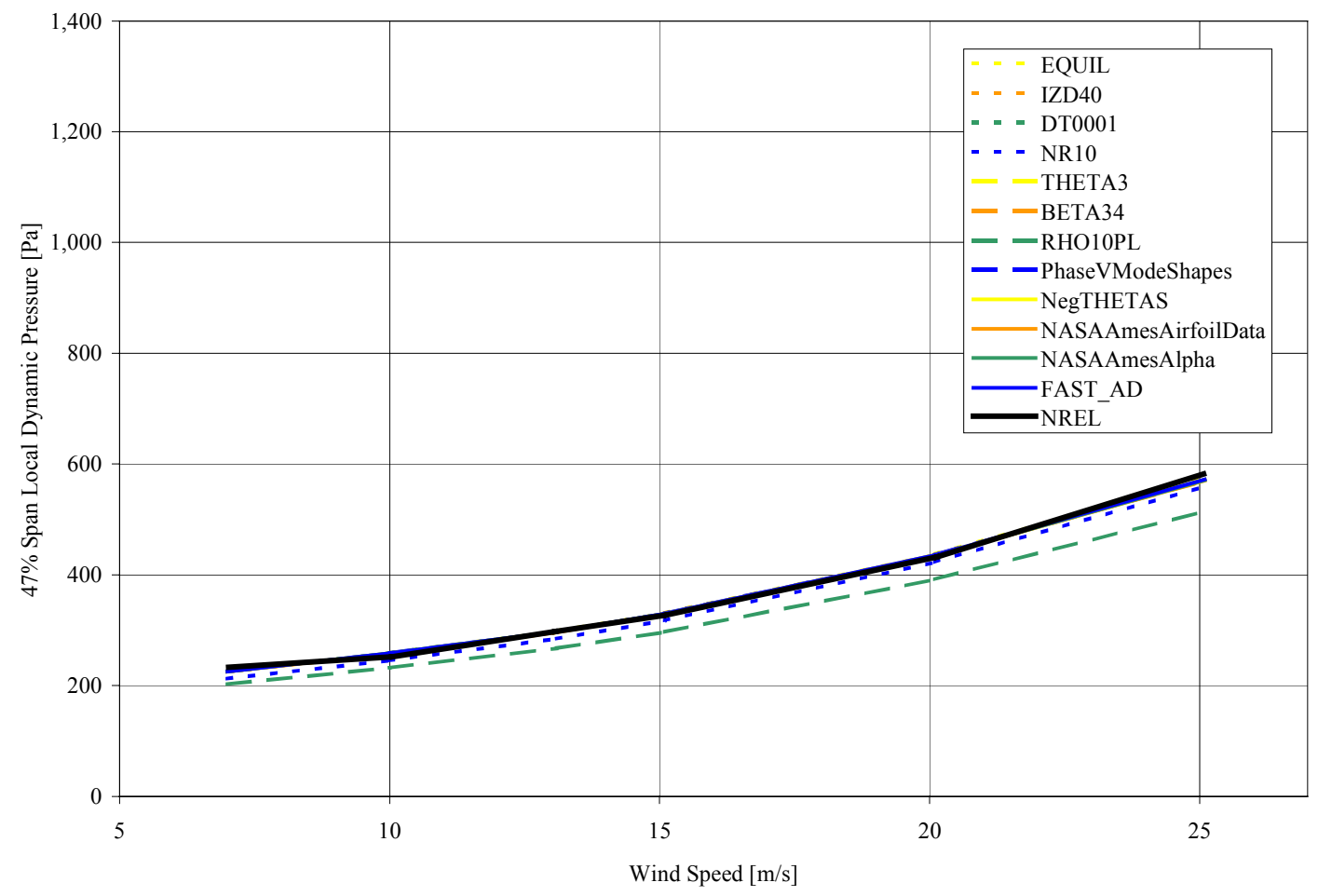

Figure $7.20: 47 \%$ span local dynamic pressure, upwind, zero yaw 


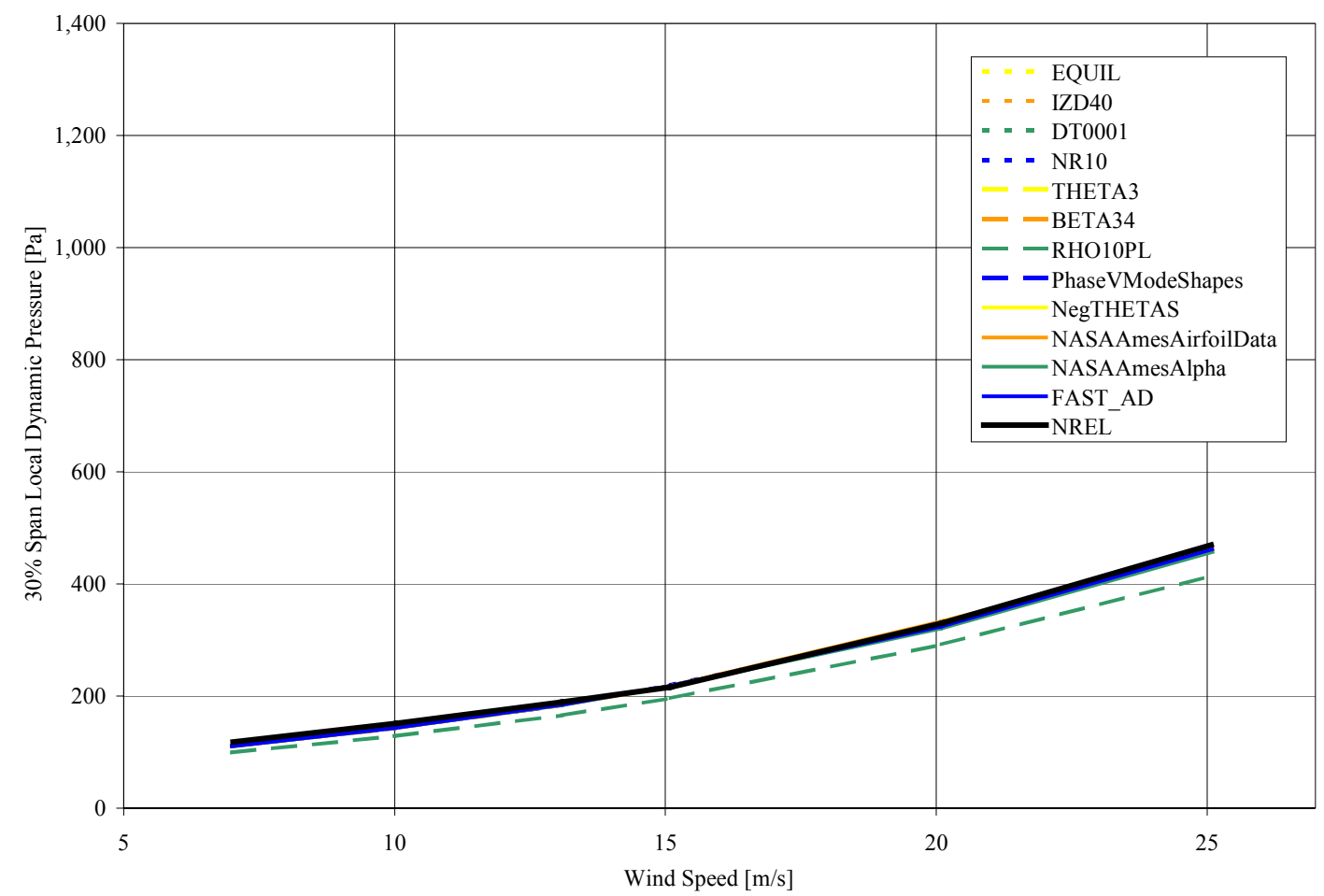

Figure 7.21: $30 \%$ span local dynamic pressure, upwind, zero yaw

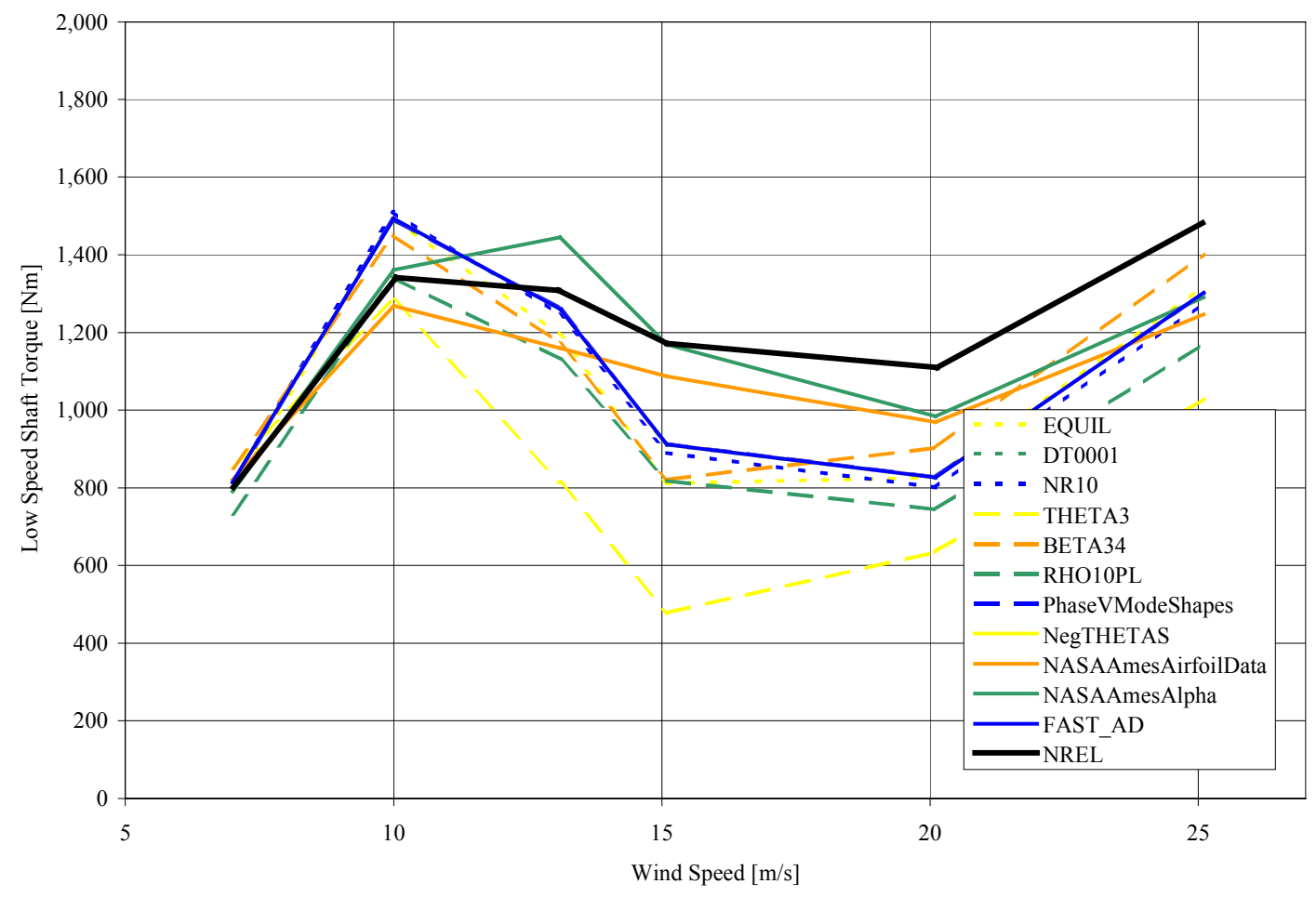

Figure 7.22: Low-speed shaft torque, upwind, zero yaw 


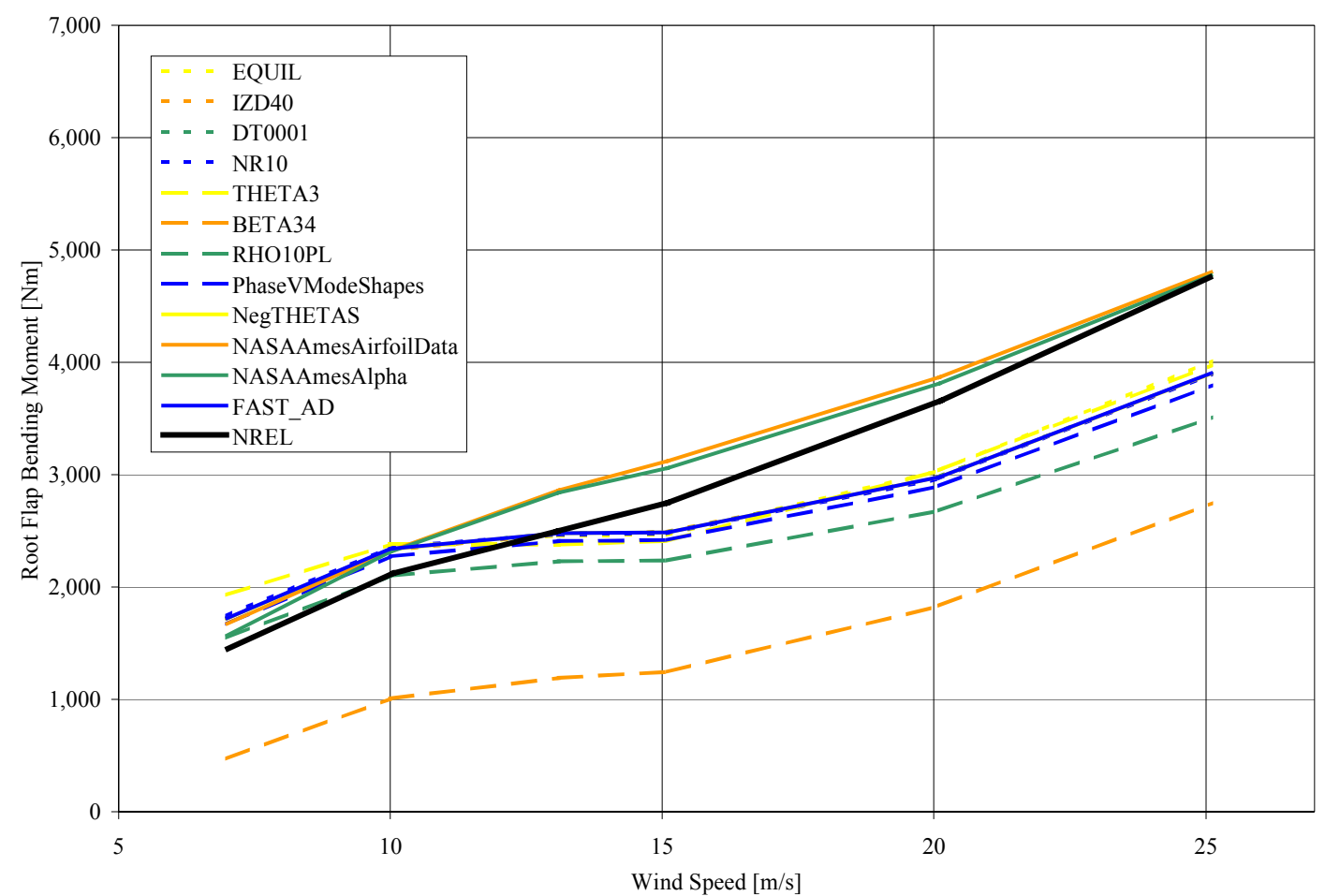

Figure 7.23: Root flap bending moment, upwind, zero yaw

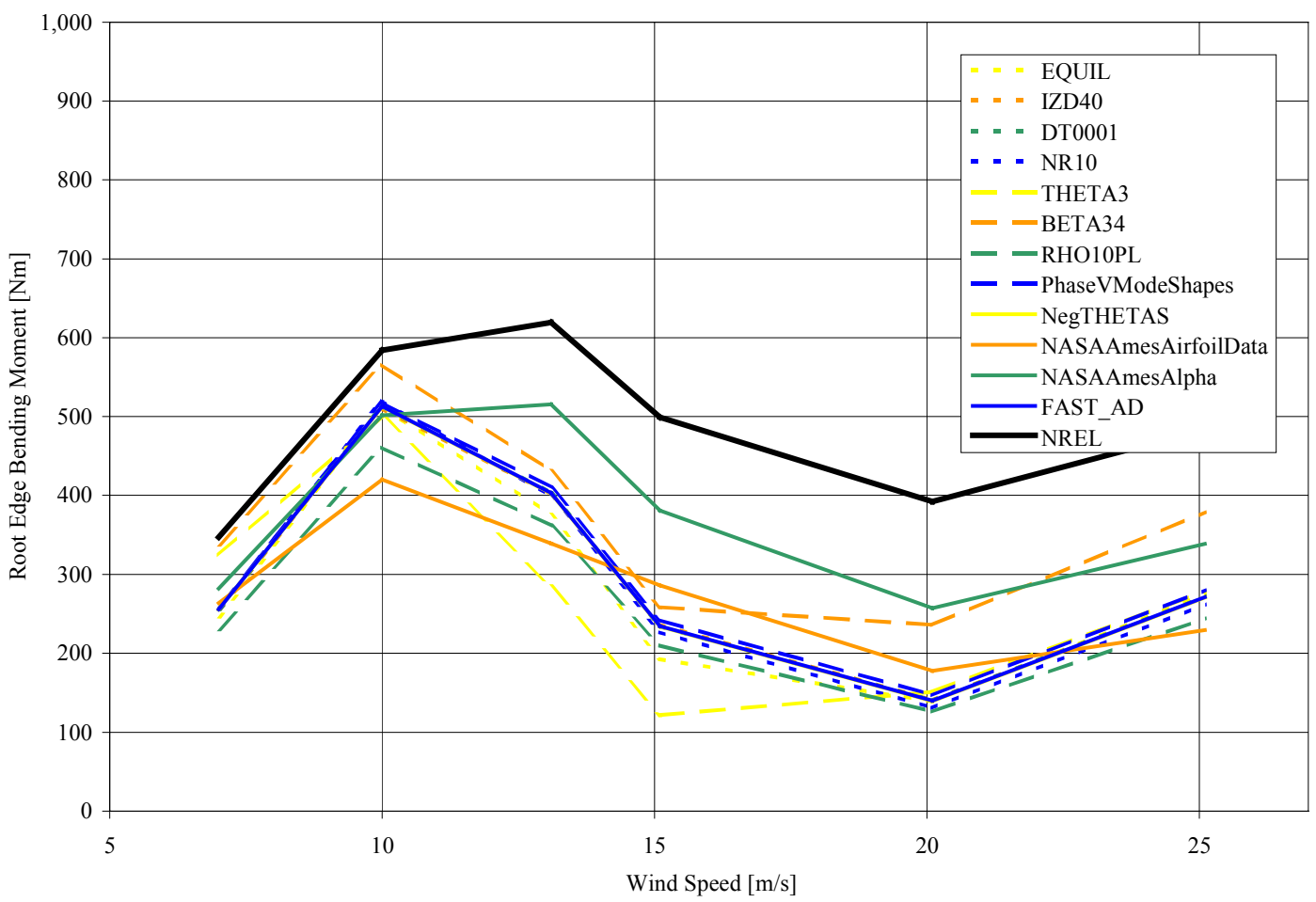

Figure 7.24: Root edge bending moment, upwind, zero yaw

Though many of the data series from the sensitivity study simulations appear to be absent from these plots, all these "invisible" curves lie directly under the curves labeled "FAST_AD." For 
example, in the "EQUIL" study, the predicted normal and tangential force coefficients at 30\% span are slightly offset from the original FAST_AD predictions for the Blind Comparison work (represented by the "FAST_AD" data series). There are also slight dissimilarities between the "EQUIL" and "FAST_AD" predictions of the low-speed shaft torque, root flap and edge bending moments, and local dynamic pressure at $95 \%$ span. However, the predictions made during the "EQUIL" and "FAST_AD" exercises of the remaining force coefficients and local dynamic pressures are nearly identical, causing the associated data series for the "EQUIL" study to coincide with the data series labeled "FAST_AD." Thus, many of these "EQUIL" curves appear invisible. Similar explanations account for the remaining "invisible" data series.

The similarity between the load predictions made via the classic BEM model ("EQUIL" study) and dynamic inflow model ("FAST_AD" study) establishes that the two theories are nearly equivalent for zero yaw error test cases. Addressing question (1) in the introduction to this chapter, we conclude that this choice of aerodynamic parameters did not contribute to any of the load prediction discrepancies among the various modelers and modeling tools in the Blind Comparison exercise for the upwind configuration, zero yaw error test cases. Furthermore, the choice of whether to employ the standard BEM model in place of the dynamic inflow model is not critical when modeling operational states where dynamic inflow effects are negligible [addressing question (2)].

For the six test cases analyzed in this sensitivity study, not once is it evident that selecting generator models (induction in "FAST_AD" or fixed speed in "IZD40") influenced the load and performance predictions. When modeling with an induction generator, rotor speed deviates by no more than $0.1 \mathrm{rpm}$ for the zero yaw error test cases (these data are not presented here). This deviation jumps up to several rpm for the nonzero yaw error test cases. Referring to question (1), the application of different generator models should not be a cause of load prediction discrepancies for the zero yaw error test cases.

There is also no visible difference between the load predictions when a time increment of $0.001 \mathrm{~s}$ (study "DT001") is used in place of $0.002 \mathrm{~s}$. An azimuth step of $1^{\circ}$, from which the original time step of $0.002 \mathrm{~s}$ was derived, appears to be appropriately, if not conservatively, selected. The time increment is thus another parameter that did not cause any performance prediction discrepancies seen in the Blind Comparison study, unless of course, a Blind Comparison participant chose a foolishly large increment.

The load predictions made during the "NR10" sensitivity study are surprisingly similar to predictions made with twice as many blade elements during the original "FAST_AD" Blind Comparison work. Discrepancies between the load predictions made by these two studies are minimal whether one is considering local force coefficient values, local dynamic pressures, or bending moments. This implies that the number of blade elements chosen by the Blind Comparison participants played an insignificant role in the discrepancies among their load predictions if they used linear interpolations to pinpoint aerodynamic loads at the correct pressure tap span stations.

Using linear interpolations to pinpoint aerodynamic loads at span stations between two adjacent nodes is equivalent to assuming that force coefficient-angle of attack relationships are linear 
between any two points where these curves intersect the BEM curves [Eq. (2.73)]. The results of the "NR10" study demonstrate that linear interpolation is an appropriate technique since the load predictions obtained with 20 blade elements are nearly identical to those made with 10.

Load predictions made during the "THETA3" study vary considerably from those made during the original "FAST_AD" Blind Comparison work. This is particularly evident for the aerodynamic force coefficients at the outboard span stations ( $80 \%$ and $95 \%$ ), the low-speed shaft torque, and the root edge bending moment at all wind speeds. Naturally, this parameter is critical for accurate load and performance predictions [regarding question (2)]. Since several Blind Comparison participants probably used $3^{\circ}$ instead of $4.815^{\circ}$ for the blade collective pitch angle relative to zero aerodynamic twist (see section 7.1), many of the load prediction discrepancies evident in the Blind Comparison exercise may result directly from this error.

Since the orientation of a blade about its central axis is critical to the resulting component loads (demonstrated by the "THETA3" study), the absence of any blade torsional motion modeling in FAST_AD may be a contributing factor in some of the load prediction inaccuracies. Conversely, data from the NASA-Ames wind tunnel tests (not presented here) show that tip pitch angles range by no more than $0.35^{\circ}$ throughout even the most extreme test conditions (high wind speed, large yaw error test cases). Consequentially, the inability to model blade torsional motion likely produced a negligible effect on the load predictions. This will probably not be the case when modeling more flexible blades, however (see Chapter 4).

There are very minor differences between aerodynamic force coefficient predictions made during the "BETA34" study and the "FAST_AD" simulations. The local dynamic pressure predictions for these two studies are nearly identical. However, the two studies have substantially different predictions of the torque and bending moments. The root flap bending moment predictions from the "BETA34" study are considerably lower than the original predictions (see Fig. 7.23). The shaft torque and bending moment output channels are highly sensitive to flapping angle perturbations; local aerodynamic loads are not. This reality is a bit puzzling. Indeed, the root flap bending moment should decrease when downwind preconing is prescribed, since centrifugal loads tend to offset thrust loads in this situation. However, the same conditions are not true for root edge bending moments or low-speed shaft torque. There is no mechanism for a small perturbation in blade coning angle to increase root edge bending moments and significantly affect low-speed shaft torque predictions. This leads to the suspicion that the method FAST_AD uses to integrate aerodynamic loads to back out bending moments may be erroneous. This suspicion is fortified in discussions at the end of this chapter.

The results of the "RHO10PL" study are not surprising. As implied in Eq. (2.73) by the absence of the fluid density $(\rho)$, computation of the operational aerodynamic force coefficients is independent of the fluid density. This is demonstrated in the results of the "RHO10PL" study; predictions of the dimensionless aerodynamic force coefficients obtained via the "RHO10PL" and "FAST_AD" simulations are identical. Also, the actual aerodynamic forces, characterized in predictions of the local dynamic pressures, low-speed shaft torque, and root flap and edge bending moments, are all scaled down proportionally with the air density. This is not surprising, since all these loads are linearly proportional to the air density [see, for example, Eq. (2.50)]. Thus, if one underpredicts air density, one will underpredict component loads by the same 
fraction. Knowledge of the environment in which a turbine is to be placed is critical in the wind turbine design and selection process.

The "PhaseVModeShapes" sensitivity study shows that the variation in blade structural properties and associated mode shapes has no effect on the predictions of local aerodynamic force coefficients and dynamic pressures. Also, these variations have only a small effect on the predictions of low-speed shaft torque and root flap and edge bending moments, key items of information when considering hub loads and blade life. Question (1) indicates the various approximations and assumptions Blind Comparison participants made with regard to the specification of blade structural properties had little influence on their load prediction discrepancies. This assertion most likely applies only since the blades of the UAE turbine are quite stiff. Measurements taken during the NASA-Ames wind tunnel tests show that actual flap angles are no larger than $0.05^{\circ}$ (these data are not presented here). Blade structural properties and mode shapes may have a greater impact on the component loads of more flexible machines.

A comparison of the "THETA3," "BETA34," "RHO10PL," and "PhaseVModeShapes" sensitivity studies shows that the parameters most directly associated with the aerodynamics computations are notably more critical than structural parameters when developing models to consider loads and performance. Factors such as mode shapes and other distributed blade structural parameters have a negligible effect on the aerodynamics and only a minor effect on the low-speed shaft torque and root flap and edge bending moments (at least on stiff turbines).

The results of the "NegTHETAS" study are unexpected. Since the change in blade structural properties and associated mode shapes has some effect on the resulting torque and moment predictions, reversing the sign of the structural pretwist distribution angles would likely affect them as well - especially since the twist distribution has a large variation (a range of approximately $22^{\circ}$ ). However, this is not the case. Just like the "DT001" study, there is absolutely no difference between the load predictions derived from the "NegTHETAS" study and those derived from the original Blind Comparison work ("FAST_AD"). This could mean that the code error uncovered in footnote 9 of Chapter 3 is inconsequential for stiff rotor blades. Or there may be some code feature in FAST_AD that takes the magnitudes instead of the actual values of the structural pretwist angles during processing. The incorrect sign of the structural pretwist angles likely has important consequences on the dynamical outcomes of the simulations. As in the "PhaseVModeShapes" study, this will probably have a greater impact on the component loads of more flexible machines.

Compared to measurements taken in the NASA-Ames wind tunnel, aerodynamic force coefficients are more accurately predicted in the "NASAAmesAirfoilData" study than they are in the original "FAST_AD" work. They are more but not entirely accurate. Prediction of aerodynamic force coefficients is highly sensitive to mispredictions in angle of attack, especially in the attached flow and stall regions. For example, when using the NASA-Ames airfoil table data, FAST_AD overpredicts the angle of attack at 63\% span at every wind speed (see Figs. 7.5 and 7.6). At a wind speed of $7 \mathrm{~m} / \mathrm{s}$, this overprediction is about $1.6^{\circ}$. Figure 7.1 demonstrates that the flow around the $63 \%$ span station is attached at this wind speed since the symbol 
associated with the $63 \%$ span, $7 \mathrm{~m} / \mathrm{s}$ wind speed measurement recording lies on the linear portion of the force coefficient curve ${ }^{31}$. Starting at this symbol, an overprediction in angle of attack of about $1.6^{\mathrm{o}}$ would cause the normal force coefficient to be overpredicted by about 0.08 . Indeed, this is exactly what is seen in Fig. 7.9. Fig. 7.1 also reveals that the $63 \%$ span radial location experiences stall sometime between a wind speed of 10 and $11 \mathrm{~m} / \mathrm{s}$. At a wind speed of $10 \mathrm{~m} / \mathrm{s}$, FAST_AD overpredicts the angle of attack by about $1.9^{\circ}$ when using the NASA-Ames airfoil table data (see Fig. 7.5 or 7.6.). As shown in Fig. 7.1, an overprediction in angle of attack of this magnitude around stall will cause the normal force coefficient to be underpredicted by about 0.2. This is seen in Fig. 7.9. Similarly, the overprediction in angle of attack at this wind speed and span station directly results in the underprediction in the tangential force coefficient (see Fig. 7.14), which is easily verified by examining the events around stall (see Fig. 7.2).

This process can be used to explain the discrepancies between the predicted and measured aerodynamic force coefficients at every span station and wind speed. Aerodynamic load predictions are more accurate at high wind speeds and several other instances since the slopes of the force coefficient-angle of attack relationships are small in these situations, and thus a slight misprediction in angle of attack will not lead to any significant misprediction of the aerodynamic force coefficient.

The inability to pinpoint the correct force coefficient-angle of attack pairs when using accurate airfoil data that take into account three-dimensional flow effect considerations, or the inability to accurate predict angle of attack in general, underscores a fundamental flaw in the classic BEM aerodynamics models. Since design tools such as FAST_AD will only significantly help to improve wind turbine designs if component loads are predicted accurately, refining or redeveloping efficient rotary wing aerodynamics models is essential. Addressing question (4), this work will have the greatest impact on wind turbine design improvement and wind-generated electricity cost reduction. When component loads are accurately predicted, safety factors may be relaxed and turbine designs can be optimized. The fact that the angle of attack mispredictions are consistent at every wind speed (see Fig. 7.5 or 7.6.) makes the feasibility of this task promising. Indeed, without modifications to any aerodynamics models, the aerodynamic load predictions made by the "NASAAmesAirfoilData" study are the most accurate that this theory can achieve. Without theory modification or the ability to use more accurate airfoil data that take three-dimensional flow into consideration, a better prediction of experimentally measured loads is impossible.

Since the aerodynamic loads predicted by the "NASAAmesAirfoilData" study are more accurate than those predicted during the "FAST_AD" study, when using airfoil data to predict aerodynamic loads on wind turbines, measuring airfoil characteristics on a rotating blade may be wiser than measuring them on a static airfoil and applying correction algorithms to account for three-dimensional flow effects. Unfortunately, the cost of adequate tests is prohibitive in the

\footnotetext{
${ }^{31}$ The data points in this figure associated with the smallest angle of attacks correspond to the data measured during the smallest wind speed test cases. Thus, the leftmost symbol for each span station data series corresponds to the 5 $\mathrm{m} / \mathrm{s}$ wind speed test case. The next symbol to the right corresponds to the $6 \mathrm{~m} / \mathrm{s}$ wind speed test case and the next symbol to the right of that corresponds to the $7 \mathrm{~m} / \mathrm{s}$ wind speed test case. This process can repeated for all 21 wind speed tests, so the rightmost symbol for each span station data series corresponds to the $25 \mathrm{~m} / \mathrm{s}$ wind speed test case.
} 
design and analysis environment. The cost to perform the NASA-Ames wind tunnel tests is feasible only in the research environment. Furthermore, the physics of flow are not well enough understood to know what similitude (scaling) parameters are necessary if rotating blade data are to be scaled to blades of a different size. Certainly testing full-size rotating blades is impractical, since most utility-scale blades cannot fit in even the world's largest wind tunnels.

A comparison of the "DT001," "NR10," and "NASAAmesAirfoilData" sensitivity studies reveals that using accurate airfoil table data is much more important than using more blade elements or using smaller time steps to integrate aerodynamic and elastic forces. This is important because significant computer-time savings can be achieved by using fewer elements and larger time steps. However, when modeling blades that are considerably more flexible, the use of more blade elements and smaller time steps will likely become more important.

As stated in section 7.1, the "NASAAmesAlpha" study is conducted to determine whether the predicted and measured aerodynamic load discrepancies account for the discrepancies between the measured low-speed shaft torque and root flap and edge bending moments and the predicted values obtained from the "NASAAmesAirfoilData" study. The "NASAAmesAlpha" study shows clearly that modifying the aerodynamic twist distribution eliminated the predicted and measured aerodynamic load discrepancies almost entirely. However, the low-speed shaft torque and root flap and edge bending moments are still mispredicted in this study! These inaccuracies are difficult to explain.

One possible explanation is that the inaccuracies in torque and bending moments may result from the absence of skin friction drag effects in the aerodynamic force coefficient data obtained from the NASA-Ames wind tunnel tests and used in the "NASAAmesAirfoilData" and "NASAAmesAlpha" studies. Since the aerodynamic force coefficients are measured using pressure transducers, which measure only the pressure drag, viscous stress effects are neglected in the measurements of the aerodynamic force coefficients obtained from the NASA-Ames wind tunnel tests. If the viscous stress contribution to the drag force could somehow be quantified, measurements of the tangential force coefficients would, in essence, decrease [an increase in drag force is a decrease in tangential force as seen in Eq. (2.42)]. Equation (2.57) predicts that a decrease in tangential force will lead to an increase in thrust force and associated root flap bending moment. Equation (2.58) predicts that a decrease in tangential force will lead to a decrease in low-speed shaft torque, or equivalently root edge bending moment. Unfortunately, these are backward to what is needed to shift the predicted torque and moment curves to the curves representing the experimentally measured loads. This thought process reveals that the inaccuracies in predicting torque and bending moments do not result from the lack of skin friction drag in the aerodynamic force coefficient data.

One possible explanation for the inaccuracy in torque and bending moment predictions obtained from the "NASAAmesAlpha" study is that aerodynamic load data are known to be accurately predicted at only five radial locations on the blade. Some of this inaccuracy may be attributable to potential mispredictions in aerodynamic loads at other span locations. This is unlikely to be the only contributing factor, however. 
Instead, errors and limitations in the structural models and load routines probably form the crucial explanation. The belief that there are errors in the structural models and load routines is reinforced by the computations given in Table 7.10. This table compares actual predicted and measured low-speed shaft torque values (LSSTQ) with "estimates" of low-speed shaft torque based on the vector sum of the root flap and edge bending moments (B3RFB and B3REB respectively). Since the root bending moments are measured fairly near to the rotor axis, the value of $L S S T Q$ can be estimated from the values of $B 3 R F B$ and $B 3 R E B$ via the following vectorial relationship:

$$
L S S T Q \approx 2\left[B 3 R E B \cos \left(3.0^{\circ}\right)+B 3 R F B \sin \left(3.0^{\circ}\right)\right]
$$

The "2" appears in Eq. (7.1) since moments from two blades give rise to the overall shaft torque. Application of this equation is demonstrated for the "NREL," "FAST_AD," and "NASAAmesAlpha" data in Table 7.10.

Table 7.10: Demonstration of Torque and Bending Moment Relationship Flaws

\begin{tabular}{|c|c|c|c|c|c|c|c|}
\hline Data Series & $\begin{array}{c}\text { Wind } \\
\text { Speed } \\
\mathrm{m} / \mathrm{s}\end{array}$ & $\begin{array}{r}B 3 R E B \\
N m\end{array}$ & $\begin{array}{r}B 3 R F B \\
N m\end{array}$ & $\begin{array}{r}*\left[B 3 R E B^{*} \cos (3)\right. \\
+B 3 R F B * \sin (3)] \\
N m\end{array}$ & $\begin{array}{r}\text { Actual } \\
L S S T Q \\
N m\end{array}$ & $\begin{array}{r}\text { Mismatch } \\
\text { Error }\end{array}$ & \begin{tabular}{r|} 
Average \\
Mismatch \\
Error
\end{tabular} \\
\hline \multirow{6}{*}{ "NREL" } & 7 & 347 & 1,453 & 845 & 801 & $5.5 \%$ & \multirow{6}{*}{$6.2 \%$} \\
\hline & 10 & 584 & 2,121 & 1,388 & 1,341 & $3.5 \%$ & \\
\hline & 13 & 619 & 2,497 & 1,498 & 1,309 & $14.4 \%$ & \\
\hline & 15 & 499 & 2,750 & 1,284 & 1,172 & $9.6 \%$ & \\
\hline & 20 & 392 & 3,658 & 1,166 & 1,110 & $5.0 \%$ & \\
\hline & 25 & 484 & 4,757 & 1,465 & 1,482 & $-1.1 \%$ & \\
\hline \multirow{6}{*}{ "FAST_AD" } & 7 & 256 & 1,721 & 691 & 815 & $-15.2 \%$ & \multirow{6}{*}{$-20.2 \%$} \\
\hline & 10 & 513 & 2,339 & 1,269 & 1,492 & $-14.9 \%$ & \\
\hline & 13 & 403 & 2,480 & 1,064 & 1,260 & $-15.6 \%$ & \\
\hline & 15 & 234 & 2,487 & 728 & 912 & $-20.2 \%$ & \\
\hline & 20 & 140 & 2,980 & 592 & 827 & $-28.4 \%$ & \\
\hline & 25 & 271 & 3,901 & 950 & 1,302 & $-27.0 \%$ & \\
\hline \multirow{6}{*}{ "NASAAmesAlpha" } & 7 & 282 & 1,571 & 728 & 791 & $-8.0 \%$ & \multirow{6}{*}{$-8.1 \%$} \\
\hline & 10 & 502 & 2,314 & 1,244 & 1,362 & $-8.6 \%$ & \\
\hline & 13 & 516 & 2,840 & 1,327 & 1,444 & $-8.1 \%$ & \\
\hline & 15 & 381 & 3,060 & 1,080 & 1,169 & $-7.6 \%$ & \\
\hline & 20 & 257 & 3,811 & 912 & 985 & $-7.4 \%$ & \\
\hline & 25 & 339 & 4,773 & 1,176 & 1,290 & $-8.9 \%$ & \\
\hline
\end{tabular}

With the NASA-Ames test data ("NREL"), the "estimated" low-speed shaft torque (column 5) overpredicts the actual measured low-speed shaft torque (column 6 ) by about $6.2 \%$ on average. In contrast, the "estimated" low-speed shaft torque using Eq. (7.1) underpredicts the value of the low-speed shaft torque obtained using FAST_AD by about $10 \%-20 \%$ on average. Intuitively speaking, an "estimated" low-speed shaft torque would underpredict the actual low-speed shaft torque, since the bending moments at the rotor that combine to form the low-speed shaft torque should be smaller at the hub radius than at the rotor axis. Why "estimated" low-speed shaft torque overpredicts actual measured low speed shaft torque with the experimental test data is unclear. Nevertheless, the results demonstrate that the relationships between the low-speed shaft torque and root bending moments are not properly modeled in FAST_AD. 
Exact identification of the errors that cause these relationship flaws is difficult without thoroughly examining the code. Code designers of FAST_AD suspect that there are errors in the loads-analysis routines. This theory is not documented here. Additional structural modeling errors that can contribute to the inaccuracies in torque and bending moment predictions include the errors uncovered in Chapter 3 and described in Chapter 4.

Limitations in the structural models of FAST_AD that can contribute to the inaccuracies in torque and bending moment predictions include the inability to model pitching moments and radial aerodynamic forces (both are also discussed in Chapters 3 and 4), since both these additional blade aerodynamic loads contribute fundamentally to the stress levels at the blade root. Radial aerodynamic loads can contribute to blade root bending moments if the loads or blade deflections are substantial. Pitching moments contribute to the deflected blade shape, which is inherently coupled with the ensuing aerodynamic loads and resulting torque and bending moments (see the earlier discussion of the "THETA3" sensitivity study).

These flaws must be pinpointed and corrected for accurate hub loading, rotor torque, and overall performance predictions. Nevertheless, the results of the sensitivity study demonstrate that until the aerodynamics models are refined or redeveloped, eradicating these flaws is less critical. Our limited understanding of the three-dimensional flow environment and the flow physics involved overwhelms most of these errors and model limitations. 


\section{Conclusions}

Researchers and designers use design codes such as FAST_AD to efficiently, safely, and costeffectively design and analyze wind energy systems. After developing aerodynamic and structural models of the UAE research wind turbine in FAST_AD, simulations are run by exciting the model with the same conditions seen in the NASA-Ames wind tunnel for several UAE test cases. To facilitate validation of FAST_AD, the load predictions are compared with those made by other similar modeling tools and with experimental measurements taken on the actual UAE wind turbine during the NASA-Ames wind tunnel tests. The experimental test data acquired from the NASA-Ames wind tunnel tests represent the finest, most accurate set of wind turbine aerodynamic and induced flow field data available. There are load magnitude discrepancies - inconsistencies in predicted and measured aerodynamic force coefficients, rotor shaft torque, and out-of-plane bending moments at the blade root-between the modeling tool predictions and experimental measurements over a range of operating conditions. .

Inconsistencies in input file parameters - variations in aerodynamic force coefficient-angle of attack characteristics, blade node positions for data recording and analysis, and pitch angle convention-explain a noteworthy fraction of the load prediction discrepancies of the various modeling tools. Furthermore, other input file parameter inconsistencies such as variations in the time step, the number of blade elements, and the distributed blade structural properties contributed little to the load prediction discrepancies. Other reasons for load prediction discrepancies include modeler error and code bugs.

Discrepancies between modeling tool load predictions and physically measured load values highlight weaknesses in tip loss, stall delay, post stall, and other frequently used aerodynamic models. A comparison of force coefficient-angle of attack relationships measured in the threedimensional environment of the NASA-Ames wind tunnel at each pressure tap grouping span station reveals that three-dimensional flow effects prohibit the use of unmodified twodimensional airfoil data at all span stations, not only the inboard stations as originally thought. These effects include spanwise pressure gradients, spanwise flow, flow circulation, and vorticity. Moreover, the inability to pinpoint angle of attack values, even when using accurate airfoil data that take into account three-dimensional flow behavior, is a fundamental fault in the classic BEM aerodynamic models.

Demonstrating mispredictions in low-speed shaft torque and root flap and edge bending moments even when mispredictions in aerodynamic forces are eliminated at all span stations uncovers flaws and limitations in the structural models employed by FAST_AD and suggests means of model and code improvement. These flaws and limitations include an improper definition of the blade structural pretwist angle and the inability to model (1) radial aerodynamic forces acting on the blades, (2) blade pitching moments, and (3) properly understanding the relationship between the low-speed shaft torque and root bending moments.

These flaws must be pinpointed and corrected for accurate hub loading, rotor torque, and overall performance predictions. This work should be completed before FAST_AD validation concludes. Nevertheless, the results demonstrate that until the aerodynamics models are refined or redeveloped, eradicating these flaws is less critical. Our limited understanding of the three- 
dimensional flow environment and the flow physics involved overwhelms most of these errors and model limitations. Aerodynamic models need to more accurately incorporate threedimensional flow effects and predict angle of attack distributions. Refining or redeveloping efficient rotor wing aerodynamics models is essential if design tools, such as FAST_AD, are to accurately predict component loads and significantly reduce wind-generated electricity costs. When component loads are accurately predicted, safety factors may be relaxed and turbine designs can be optimized without the fear of detrimental component failure. The consistency of the angle of attack mispredictions at every wind speed makes the feasibility of this task promising. 


\section{References}

Buhl, M.L. WT_Perf User's Guide, National Renewable Energy Laboratory, 2000.

Du, Z. and Selig, M.S. "A 3-D Stall-Delay Model for Horizontal Axis Wind Turbine Performance Prediction," Proceedings of the 1998 ASME Wind Energy Symposium, Reno, NV: pp. 9-19, 1998.

Eggleston, D.M. and Stoddard, F.S. Wind Turbine Engineering Design, Van Nostrand Rheinhold, New York, 1987.

Hand, M.M. NASA-Ames Wind Tunnel Test Home Page. Golden, CO: National Renewable Energy Laboratory (accessed November 2000-September 2001),

$<$ http://wind2.nrel.gov/amestest/>.

Hand, M.M.; Simms, D.A.; Fingersh, L.J.; Jager, D.W.; Cotrell, J.R.; Schreck, S.J.; Larwood, S.M. Unsteady Aerodynamics Experiment Phase VI: Wind Tunnel Test Configurations and Available Data Campaigns, NREL/TP-500-29955; Golden, CO: National Renewable Energy Laboratory, 2001.

Hansen, A.C. and Laino, D.J. User's Guide to the Wind Turbine Dynamics Computer Programs YawDyn and AeroDyn for ADAMS ${ }^{\circledR}$, University of Utah, 1998.

Kane, T.R. and Levinson, D.A. Dynamics: Theory and Applications, McGraw-Hill Inc., New York, 1985.

Leishman, J.G. and Beddoes, T.S. "A Semi-Empirical Model for Dynamic Stall," Journal of the American Helicopter Society, Volume 34(3): pp. 3-17, 1989.

McGowan, J. and Manwell, J. Wind Energy Engineering Fundamentals Course: Course Notes - Part 1, University of Massachusetts, Amherst, MA: Renewable Energy Research Laboratory, 2000.

Simms, D.A., Hand, M.M., Fingersh, L.J., and Jager, D.W. Unsteady Aerodynamics Experiment Phases II-IV Test Configurations and Available Data Campaigns, NREL/TP-500-25950;

Golden, CO: National Renewable Energy Laboratory, 1999.

Simms, D.A., Schreck, S.J., Hand, M.M., and Fingersh, L.J. NREL Unsteady Aerodynamics Experiment in the NASA-Ames Wind Tunnel: A Comparison of Predictions to Measurements, NREL/TP-500-29494; Golden, CO: National Renewable Energy Laboratory, 2001.

Suzuki, A. and Hansen, A.C. "Dynamic Inflow Model for YawDyn," Proceedings of the 1998 AWEA Windpower Conference, Bakersfield, CA: pp. 233-240, 1998.

Thomson, W.T. and Dahleh, M.D. Theory of Vibrations with Applications, $5^{\text {th }}$ Edition, PrenticeHall, Inc., New Jersey, 1998. 
Viterna, L.A. and Corrigan, R.D. "Fixed Pitch Rotor Performance of Large Horizontal Axis Wind Turbines," Proceedings, Workshop on Large Horizontal Axis Wind Turbines, NASA CP2230, DOE Publication CONF-810752, NASA Lewis Research Center, Cleveland, OH: pp. 69$85,1981$.

Wilson, R.E., Lissaman, P.B.S., and Walker, S.N. Aerodynamic Performance of Wind Turbines, Oregon State University, 1976.

Wilson, R.E., Walker, S.N., and Heh, P. Technical and User's Manual for the FAST_AD Advanced Dynamics Code, Oregon State University, 1999. 


\section{Appendix I. FAST_AD Input File for Test Case S0700000}

S0700000.inp

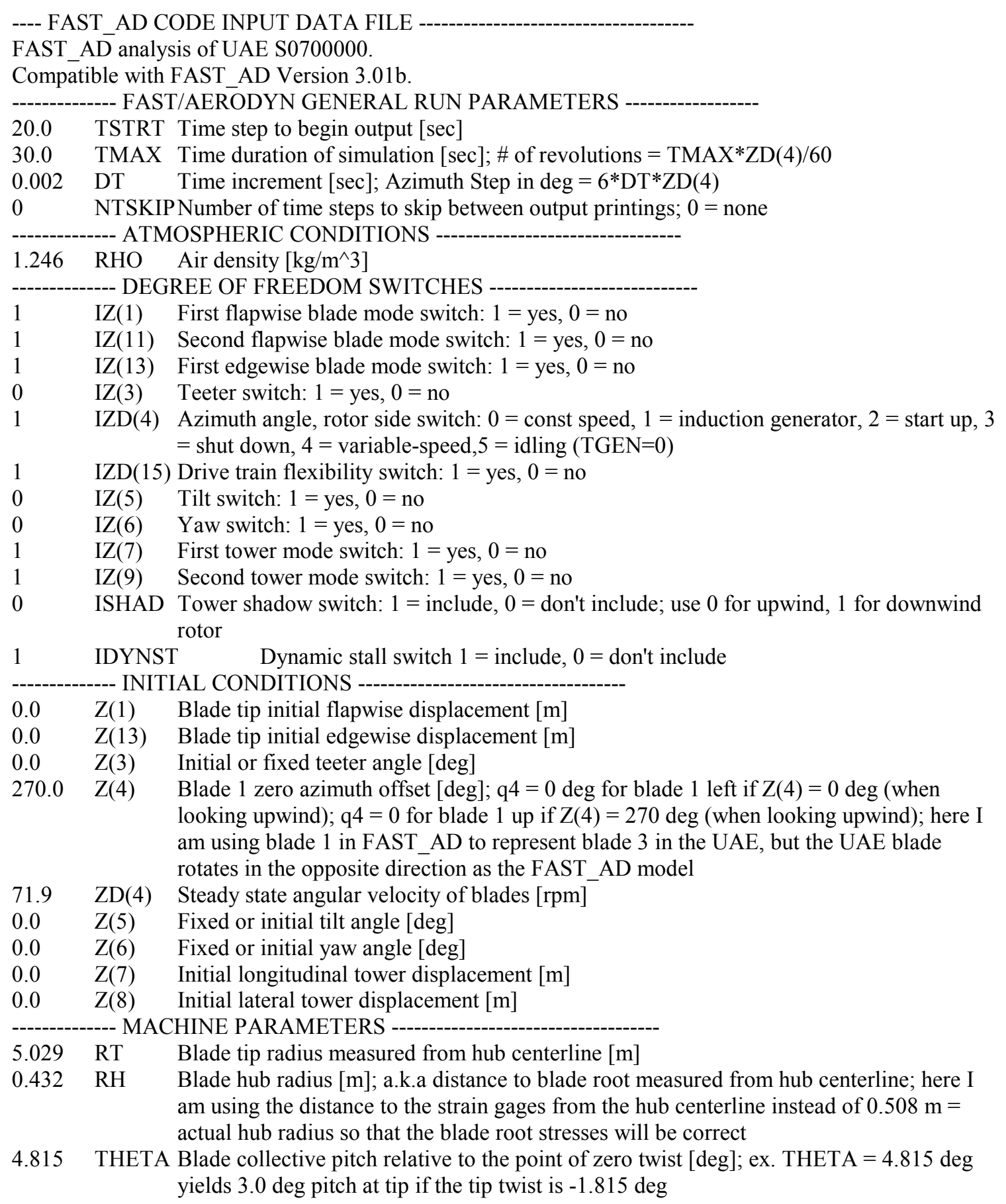




\begin{tabular}{|c|c|c|}
\hline 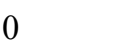 & PITCHMODE & Pitch control mode switch: $0=$ fixed pitch, $1=$ power control, $2=$ speed control \\
\hline 0.0 & STRTPTCH & Time to start pitch control [sec] \\
\hline 0.0 & \multicolumn{2}{|c|}{$\begin{array}{l}\text { RRGAP Location of end gap between blade and partial-span aileron control device in fraction of } \\
\text { tip radius [dimensionless]; use } 0.0 \text { if entire blade pitches }\end{array}$} \\
\hline 0.0 & \multicolumn{2}{|c|}{ RLU Undersling length = distance from teeter pin to blade axis intersection $[\mathrm{m}]$; this is used in } \\
\hline 0.0 & \multicolumn{2}{|c|}{ RLUM Distance from teeter pin to hub center of mass $[\mathrm{m}]$} \\
\hline-1.401 & \multicolumn{2}{|c|}{ DN Distance from yaw axis to teeter pin $[\mathrm{m}] ; \mathrm{DN}$ is $<0$ for an upwind rotor } \\
\hline 0.0 & \multicolumn{2}{|c|}{ DNM1 Distance to nacelle center of mass from yaw axis in $\mathrm{c} 1$ direction (along lo } \\
\hline 0.0 & \multicolumn{2}{|l|}{ DNM2 } \\
\hline 0.0 & \multicolumn{2}{|c|}{ SHAFTMOMLENGTH Dis } \\
\hline 12.192 & \multicolumn{2}{|c|}{$\mathrm{HH} \quad$ Hub height above ground level $[\mathrm{m}]$} \\
\hline & \multirow{2}{*}{\multicolumn{2}{|c|}{$\begin{array}{l}\text { HS Rigid base height of tower above ground level [m] } \\
\text { TWRHTOFFSETDistance from actual tower-top (yaw bearing) to rotor axis (hub height) [m] }\end{array}$}} \\
\hline 0.692 & & \\
\hline 0.0 & DELTA3 & $\begin{array}{l}\text { Delta } 3 \text { angle }[\mathrm{deg}] \text {; orients the teeter hinge so that it is no longer perpendicular } \\
\text { to the unconed blade axis }\end{array}$ \\
\hline 0.0 & BETA(1) & Blade 1 coning angle $[\mathrm{deg}]$ \\
\hline 0.0 & BETA(2) & Blade 2 coning angle [deg] \\
\hline & \multicolumn{2}{|c|}{--- MASS AND INERTIA -- } \\
\hline 1712.0 & XMNAC & $\begin{array}{l}\text { Nacelle lumped mass }[\mathrm{kg}] \text {; here I am using the UAE Nacelle }+ \text { hub }+ \text { boom }+ \\
\text { pitch shaft mass here and am setting XMHUB }=0\end{array}$ \\
\hline 0.0 & \multicolumn{2}{|c|}{ XMHUB $\quad$ Mass of hub $[\mathrm{kg}]$} \\
\hline 0.0 & \multicolumn{2}{|c|}{ TIPM(1)Mass of tip brake, blade $1[\mathrm{~kg}]$} \\
\hline 0.0 & \multicolumn{2}{|c|}{ TIPM(2)Mass of tip brake, blade $2[\mathrm{~kg}]$} \\
\hline 0.0 & HTINER & $\begin{array}{l}\text { Inertia of nacelle about tilt axis }\left[\mathrm{kg} \mathrm{m}^{\wedge} 2\right] \text {; this data is unavailable for the UAE } \\
\text { but shouldn't matter since } \mathrm{IZ}(5)=0 \text { (no tilt DOF) }\end{array}$ \\
\hline 161.5 & HSINER & $\begin{array}{l}\text { Inertia of generator (entire equivalent power train) }\left[\mathrm{kg} \mathrm{m}^{\wedge} 2\right] \text {; here I used an } \\
\text { average value from the } 144-179 \text { range }\end{array}$ \\
\hline 3789.0 & HYINER & $\begin{array}{l}\text { Inertia of nacelle about yaw axis }\left[\mathrm{kg} \mathrm{m}^{\wedge} 2\right] ; \text { here } \mathrm{I} \text { am using the UAE Nacelle }+ \\
\text { hub }+ \text { boom inertia here and am setting HINER }=0\end{array}$ \\
\hline 0.0 & \multicolumn{2}{|c|}{ HINER Inertia of hub about teeter axis [kg m^2] } \\
\hline & \multirow{2}{*}{\multicolumn{2}{|c|}{ 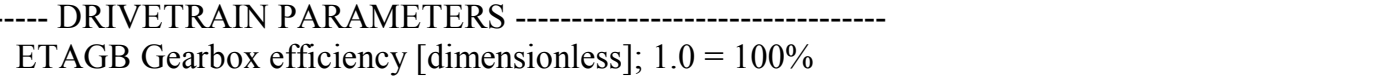 }} \\
\hline 1.0 & & \\
\hline 0.78 & ETAGEN & $\begin{array}{l}\text { Generator efficiency [dimensionless]; } 1.0=100 \% \text {; here I included the combined } \\
\text { gearbox and generator efficiency }\end{array}$ \\
\hline 72.82 & OMEGR & Rated speed for induction generator on low speed shaft side [rpm] \\
\hline 71.63 & \multicolumn{2}{|c|}{$\begin{array}{l}\text { OMEG0 Reference s } \\
{[\text { rpm] }}\end{array}$} \\
\hline 32.7539 & 9 CINGEN & Induction generator cons \\
\hline 25.13 & Gearbo & $x$ ratio [Dimensionless] \\
\hline 0.0 & Fixed 1 & ss constant $[\mathrm{N} \mathrm{m}]$ \\
\hline 0.0 & Variab & loss constant $[\mathrm{N} \mathrm{m}]$ \\
\hline 115.0 & QBRAKE & Mechanical brake torque value $[\mathrm{N} \mathrm{m}]$ \\
\hline 88.9574 & QMOTOR & Motor start-up torque (generator side) $[\mathrm{N} \mathrm{m}]$ \\
\hline $1.99 \mathrm{E} 5$ & $\begin{array}{l}\text { ZKDRV Equiv } \\
\text { high-s }\end{array}$ & $\begin{array}{l}\text { ent drive train torsional spring }[\mathrm{N} \mathrm{m} / \mathrm{rad}] \text {; includes low-speed shaft, gearbox, and } \\
\text { eed shaft }\end{array}$ \\
\hline 767.138 & $\begin{array}{l}\text { CDRV Equiva } \\
\text { gearbo }\end{array}$ & $\begin{array}{l}\text { ent drive train torsional damper }[\mathrm{N} \mathrm{m} /(\mathrm{rad} / \mathrm{sec})] \text {; includes low-speed shaft, } \\
\text {, and high-speed shaft }\end{array}$ \\
\hline 9999.9 & TGOFF Time t & turn off generator for braking [sec] \\
\hline 9999.9 & TBRAKON & Time to turn on brake [sec] \\
\hline 9999.9 & TBRAKFUL & Time at which brake reaches full torque [sec] \\
\hline 9999.9 & TIPBRAK1 & Time to apply tip brake at shut-down process [sec] \\
\hline 9999.9 & TIPBRAK2 & Time to reach the full $\mathrm{Cd}$ values of tip brake [sec] \\
\hline
\end{tabular}




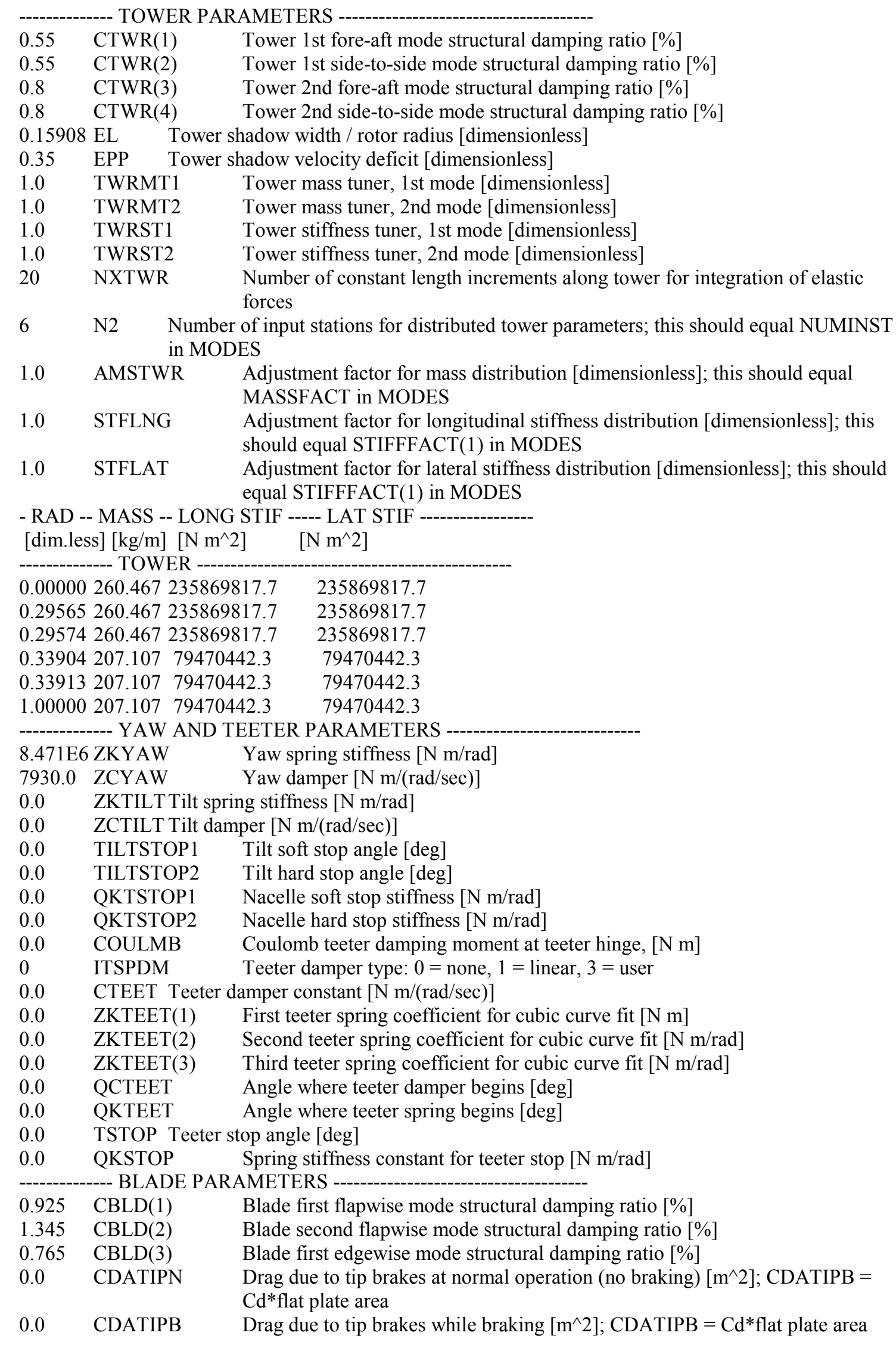




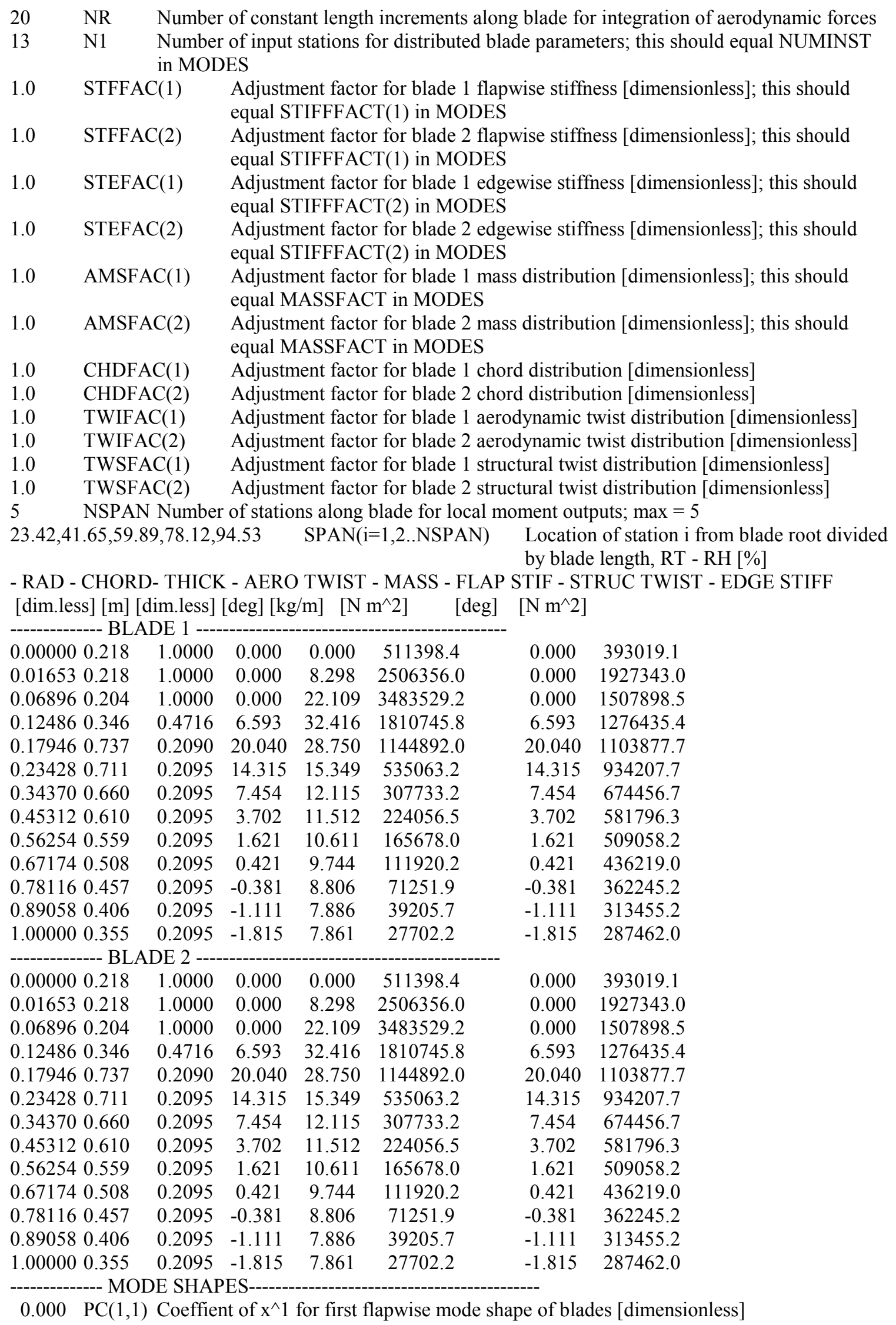




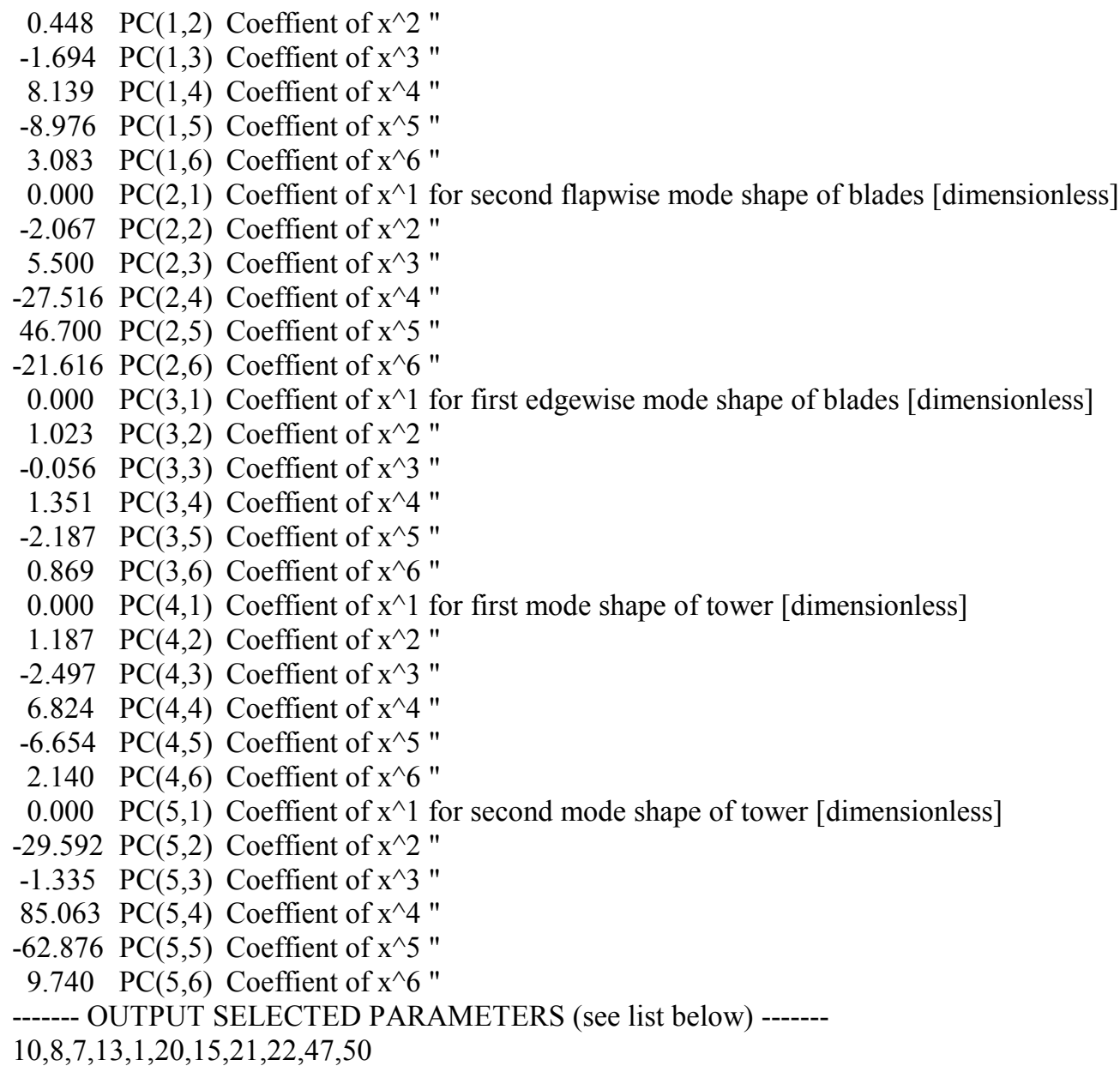

SWIRL Induction Factor Model: NONE, WAKE, SWIRL

0.005 ATOLER Tolerance for induction factor convergence; use 0.005

DYNIN Dynamic inflow model: DYNIN, EQUILibrium

$\mathrm{HH} \quad$ Wind data file type: $\mathrm{HH}=$ hub height, $\mathrm{FF}=$ full field S0700000.WND

8 NUMFOIL Number of different airfoil tables that will be read .. AirfoilDatalcylinder.dat

.. AirfoilData $\backslash$ S809_CLN_129.DAT

...AirfoilData $\backslash$ S809_CLN_185.DAT

..AirfoilData $\backslash$ S809_CLN_242.DAT

..AirfoilData $\$$ S809_CLN_298.DAT

..AirfoilData $\backslash$ S809 CLN 354.DAT

..AirfoilData $\$$ S809_CLN_410.DAT

..AirfoilDatalS809_CLN_Outboard.DAT

1 NOPRINT $\quad$ NFOIL; PRINT or NOPRINT; NFOIL = Airfoil ID number for each element

2 NOPRINT

$3 \quad$ NOPRINT

4 NOPRINT

4 PRINT

5 PRINT

6 NOPRINT

7 NOPRINT 


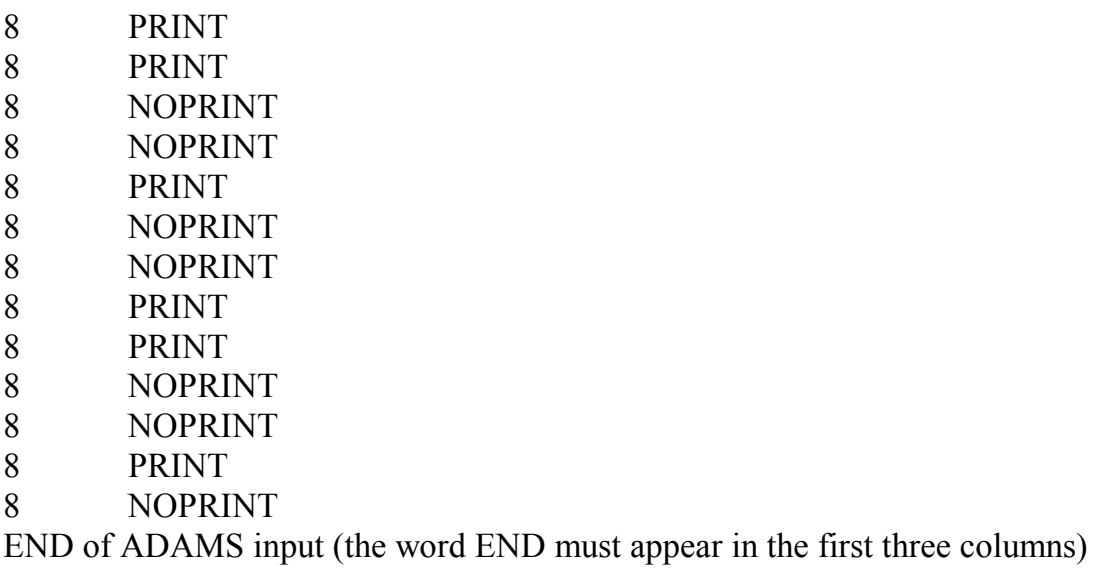




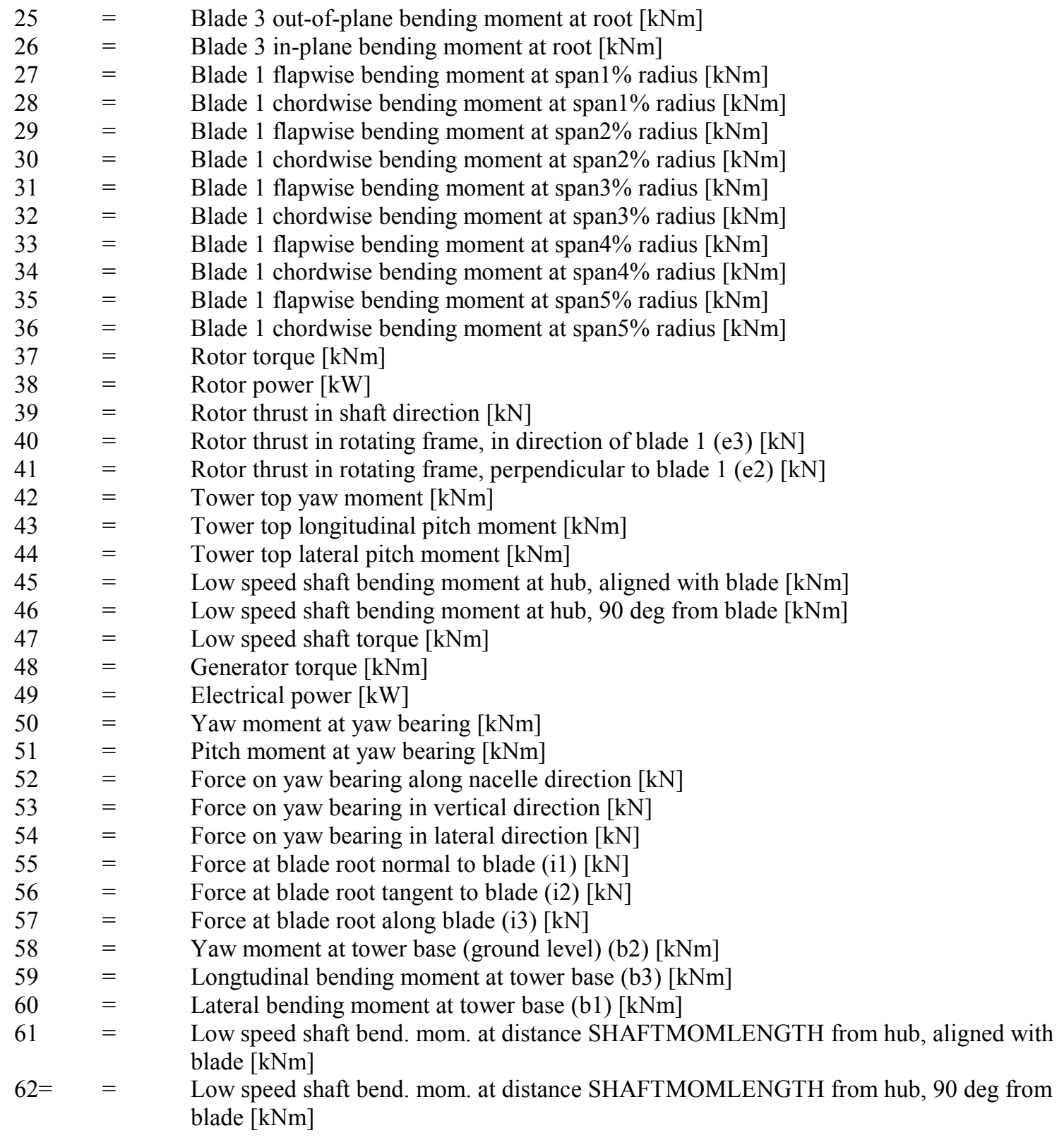




\title{
Appendix II. Modes Input Files for use in FAST_AD
}

\author{
UAE_TOWER.inp \\ False ISBLADE $\quad$ Blade/tower switch: True = Blade, False $=$ Tower \\ 0.0 OMEGA Steady-state angular velocity of rotor [rpm]; ignored for towers \\ 0.0 PITCH Blade collective pitch relative to the point of zero structural twist [deg]; ignored for \\ towers \\ 11.5 TOTLEN Total length of the beam [m]; RT for blades, HH - TWRHTOFFSET for towers \\ 0.0 RIGLEN Rigid length of the beam [m]; RH for blades, HS for towers \\ 1832.0 ENDMASS Lumped mass at the end of the beam [kg]; tip brake for blades, nacelle + rotor \\ mass for towers \\ $5 \quad \mathrm{~N} \quad$ Number of modes shapes to compute $=$ number of polynomial coefficients to use \\ $2 \quad$ NP Order of first polymomial coefficient (i.e. $\mathrm{x}^{\wedge} \mathrm{NP}$ ) \\ 6 NUMINST Number of input stations for distributed parameters \\ 1.0 MASSFACT Adjustment factor for mass distribution [dimensionless] \\ 1.0 STIFFFACT(1) Adjustment factor for out-of-plane (flapwise) stiffness distribution \\ [dimensionless] \\ 1.0 STIFFFACT(2) Adjustment factor for in-plane (edgewise) stiffness distribution [dimensionless]; \\ ignored for towers (use STIFFFACT(1))
}

0.00000260 .467235869817 .7

0.29565260 .467235869817 .7

0.29574260 .467235869817 .7

$0.33904207 .107 \quad 79470442.3$

$0.33913207 .107 \quad 79470442.3$

$1.00000207 .107 \quad 79470442.3$

---- COMMENTS (The remainder of this file is not used) ----

\section{NOTES FOR BLADES:}

If you intend to export results from Modes to FAST_AD, you must set both the structural twist and pitch to zero. FAST_AD needs the mode shapes in the local principal reference system. It will convert the shapes to in-plane and out-of-plane coordinates itself.

For the lines of distributed parameters, the first column is the fractional distance along the flexible portion of the blade. It must go from 0.0 to 1.0 . The second and third columns are the structural twist [deg] and lineal density $[\mathrm{kg} / \mathrm{m}]$. The third and fourth columns are the flatwise and edgewise stiffnesses [ $\left.\mathrm{N} \mathrm{m}^{\wedge} 2\right]$.

NOTE FOR TOWERS:

For the lines of distributed parameters, the first column is the fractional distance along the flexible portion of the tower. It must go from 0.0 to 1.0. The second column is the lineal density $[\mathrm{kg} / \mathrm{m}]$ and the third column is the stiffnesses [ $\left.\mathrm{N} \mathrm{m}^{\wedge} 2\right]$. 


\begin{tabular}{|c|c|c|c|c|}
\hline$\overline{T r u e}$ & \multirow{2}{*}{\multicolumn{2}{|c|}{$\begin{array}{l}\text { ISBLADE } \\
\text { OMEGA }\end{array}$}} & \multicolumn{2}{|c|}{ Blade/tower switch: True $=$ Blade, False $=$ Tower } \\
\hline 72.0 & & & Steady-stat & ular velocity of rotor [rpm]; ignored for towers \\
\hline 0.0 & \multicolumn{4}{|c|}{$\begin{array}{l}\text { PITCH Blade collective pitch relative to the point of zero structural twist [deg]; ignored for } \\
\text { towers }\end{array}$} \\
\hline 5.029 & \multicolumn{2}{|c|}{ TOTLEN } & \multicolumn{2}{|c|}{ Total length of the beam [m]; RT for blades, HH - TWRHTOFFSET for towers } \\
\hline 0.432 & \multicolumn{2}{|c|}{ RIGLEN } & \multicolumn{2}{|c|}{ Rigid length of the beam [m]; RH for blades, HS for towers } \\
\hline 0.0 & \multicolumn{2}{|c|}{ ENDMASS } & \multicolumn{2}{|c|}{$\begin{array}{l}\text { Lumped mass at the end of the beam }[\mathrm{kg}] \text {; tip brake for blades, nacelle }+ \text { rotor } \\
\text { mass for towers }\end{array}$} \\
\hline 5 & $\mathrm{~N}$ & \multicolumn{3}{|c|}{ Number of modes shapes to compute $=$ number of polynomial coefficients to use } \\
\hline 2 & \multicolumn{4}{|c|}{$\mathrm{NP} \quad$ Order of first polymomial coefficient (i.e. $x^{\wedge} \mathrm{NP}$ ) } \\
\hline 13 & \multicolumn{2}{|c|}{ NUMINST } & \multicolumn{2}{|c|}{ Number of input stations for distributed parameters } \\
\hline 1.0 & \multicolumn{2}{|c|}{ MASSFACT } & \multicolumn{2}{|c|}{ Adjustment factor for mass distribution [dimensionless] } \\
\hline 1.0 & \multicolumn{2}{|c|}{ STIFFFACT(1) } & \multicolumn{2}{|c|}{$\begin{array}{l}\text { Adjustment factor for out-of-plane (flapwise) stiffness distribution } \\
\text { [dimensionless] }\end{array}$} \\
\hline 1.0 & \multicolumn{2}{|c|}{ STIFFFACT(2) } & \multicolumn{2}{|c|}{$\begin{array}{l}\text { Adjustment factor for in-plane (edgewise) stiffness distribution [dimensionless]; } \\
\text { ignored for towers (use STIFFFACT(1)) }\end{array}$} \\
\hline 0.00000 & 0.0 & 0.000 & 511398.4 & ( \\
\hline 0.01653 & 0.0 & 8.298 & 2506356.0 & 1927343.0 \\
\hline 0.06896 & & 22.109 & 3483529.2 & 1507898.5 \\
\hline 0.12486 & & 32.416 & 1810745.8 & 1276435.4 \\
\hline 0.17946 & 0.0 & 28.750 & 1144892.0 & 1103877.7 \\
\hline 0.23428 & 0.0 & 15.349 & 535063.2 & 934207.7 \\
\hline 0.34370 & & 12.115 & 307733.2 & 674456.7 \\
\hline 0.45312 & & 11.512 & 224056.5 & 581796.3 \\
\hline 0.56254 & 0.0 & 10.611 & 165678.0 & 509058.2 \\
\hline 0.67174 & & 9.744 & 111920.2 & 436219.0 \\
\hline 0.78116 & 0.0 & 8.806 & 71251.9 & 362245.2 \\
\hline 0.89058 & & 7.886 & 39205.7 & 313455.2 \\
\hline 1.00000 & 0.0 & 7.861 & 27702.2 & 287462.0 \\
\hline \multicolumn{5}{|c|}{--- COMMENTS (The remainder of this file is not used) ---- } \\
\hline \multicolumn{5}{|c|}{$\begin{array}{l}\text { NOTES FOR BLADES: } \\
\text { If you intend to export results from Modes to FAST_AD, you must } \\
\text { set both the structural twist and pitch to zero. FAST_AD needs the mode } \\
\text { shapes in the local principal reference system. It will convert the } \\
\text { shapes to in-plane and out-of-plane coordinates itself. }\end{array}$} \\
\hline \multicolumn{5}{|c|}{$\begin{array}{l}\text { For the lines of distributed parameters, the first column is the } \\
\text { fractional distance along the flexible portion of the blade. It must go } \\
\text { from } 0.0 \text { to } 1.0 \text {. The second and third columns are the structural twist }[\mathrm{deg}] \\
\text { and lineal density }[\mathrm{kg} / \mathrm{m}] \text {. The third and fourth columns are the } \\
\text { flatwise and edgewise stiffnesses }\left[\mathrm{N} \mathrm{m}^{\wedge} 2\right] \text {. }\end{array}$} \\
\hline \multicolumn{5}{|c|}{$\begin{array}{l}\text { NOTE FOR TOWERS: } \\
\text { For the lines of distributed parameters, the first column is the fractional } \\
\text { distance along the flexible portion of the tower. It must go from } 0.0 \text { to } 1.0 \text {. } \\
\text { The second column is the lineal density }[\mathrm{kg} / \mathrm{m}] \text { and the third column is the } \\
\text { stiffnesses }\left[\mathrm{N} \mathrm{m}^{\wedge} 2\right] \text {. }\end{array}$} \\
\hline
\end{tabular}




\section{Appendix III. Wind Data File for Test Case S0700000}

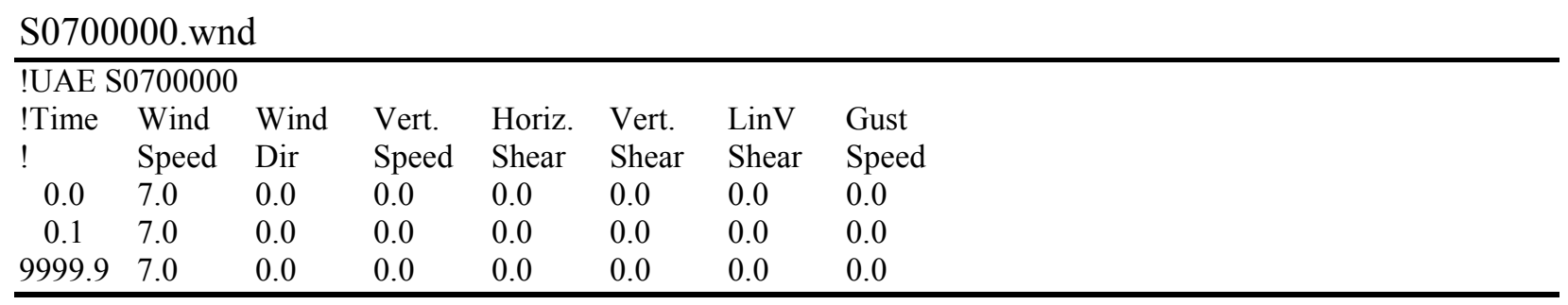




\title{
Appendix IV. Airfoil Data Table Files of the S809 Airfoil
}

\author{
cylinder.dat \\ Title or comment line number 1 \\ Title or comment line number 2 \\ 1 Number of airfoil tables in this file \\ .00 Table ID parameter \\ 150.00 Stall angle (deg) \\ .00 No longer used, enter zero \\ .00 No longer used, enter zero \\ .00 No longer used, enter zero \\ .00 Zero lift angle of attack (deg) \\ $.00000 \quad$ Cn slope for zero lift (dimensionless) \\ $.0000 \quad \mathrm{Cn}$ at stall value for positive angle of attack \\ $-8000 \quad \mathrm{Cn}$ at stall value for negative angle of attack \\ $.0000 \quad$ Angle of attack for minimum CD (deg) \\ $.3000 \quad$ Minimum $C D$ value \\ $\begin{array}{lll}-180.00 & .000 & .3000\end{array}$ \\ $\begin{array}{lll}.00 & .000 \quad .3000\end{array}$ \\ $180.00 \quad .000 \quad .3000$ \\ S809_CLN_129.dat \\ S809 Airfoil, OSU data at $\mathrm{Re}=.75$ Million, Clean roughness \\ Adjusted for Post Stall at $\mathrm{r} / \mathrm{R}=.129 * *$ \\ $1 \quad$ Number of airfoil tables in this file \\ .00 Table ID parameter \\ 30.00 Stall angle (deg)** \\ .00 No longer used, enter zero \\ .00 No longer used, enter zero \\ .00 No longer used, enter zero \\ -.38 Zero lift angle of attack (deg) \\ 7.12499 Cn slope for zero lift (dimensionless) \\ $3.50 \mathrm{Cn}$ at stall value for positive angle of attack** \\ $-.8000 \quad \mathrm{Cn}$ at stall value for negative angle of attack \\ $2.0000 \quad$ Angle of attack for minimum CD (deg) \\ $.0116 \quad$ Minimum $C D$ value \\ $\begin{array}{llll}-180.00 & .000 & .1748 & .0000\end{array}$ \\ $\begin{array}{llll}-170.00 & .230 & .2116 & .4000\end{array}$ \\ $\begin{array}{llll}-160.00 & .460 & .3172 & .1018\end{array}$ \\ $\begin{array}{llll}-150.00 & .494 & .4784 & .1333\end{array}$ \\ $\begin{array}{llll}-140.00 & .510 & .6743 & .1727\end{array}$ \\ $\begin{array}{llll}-130.00 & .486 & .8799 & .2132\end{array}$ \\ $\begin{array}{llll}-120.00 & .415 & 1.0684 & .2498\end{array}$ \\ $\begin{array}{llll}-110.00 & .302 & 1.2148 & .2779\end{array}$ \\ $\begin{array}{llll}-100.00 & .159 & 1.2989 & .2933\end{array}$ \\ $\begin{array}{llll}-90.00 & .000 & 1.3080 & .2936\end{array}$ \\ $\begin{array}{llll}-80.00 & -.159 & 1.2989 & .2933\end{array}$ \\ $\begin{array}{llll}-70.00 & -.302 & 1.2148 & .2779\end{array}$
}




\begin{tabular}{llll}
-60.00 & -.415 & 1.0684 & .2498 \\
-50.00 & -.486 & .8799 & .2132 \\
-40.00 & -.510 & .6743 & .1727 \\
-30.00 & -.494 & .4784 & .1333 \\
-20.10 & -.560 & .3027 & .0612 \\
-18.10 & -.670 & .3069 & .0904 \\
-16.10 & -.790 & .1928 & .0293 \\
-14.20 & -.840 & .0898 & -.0090 \\
-12.20 & -.700 & .0553 & -.0045 \\
-10.10 & -.630 & .0390 & -.0044 \\
-8.20 & -.560 & .0233 & -.0051 \\
-6.10 & -.640 & .0131 &. .0018 \\
-4.1 & -0.429 & 0.0134 & -.0216 \\
-2.1 & -0.202 & 0.0119 & -.0282 \\
0.1 & 0.059 & 0.0122 & -.0346 \\
2 & 0.294 & 0.0116 & -.0405 \\
4.1 & 0.539 & 0.0144 & -.0455 \\
6.2 & 0.789 & 0.0146 & -.0507 \\
8.1 & 0.970 & 0.0162 & -.0404 \\
10.2 & 1.134 & 0.0274 & -.0321 \\
11.3 & 1.210 & 0.0303 & -.0281 \\
12.1 & 1.279 & 0.0369 & -.0284 \\
13.2 & 1.375 & 0.0509 & -.0322 \\
14.2 & 1.455 & 0.0648 & -.0361 \\
15.3 & 1.539 & 0.0776 & -.0363 \\
16.3 & 1.604 & 0.0917 & -.0393 \\
17.1 & 1.639 & 0.0994 & -.0398 \\
18.1 & 1.676 & 0.2306 & -.0983 \\
19.1 & 1.691 & 0.3142 & -.1242 \\
20.1 & 1.748 & 0.3186 & -.1155 \\
30 & 2.484 & 0.4784 & -.2459 \\
40 & 2.459 & 0.6743 & -.2813 \\
50 & 2.133 & 0.8799 & -.3134 \\
60 & 1.599 & 1.0684 & -.3388 \\
70 & 0.971 & 1.2148 & -.3557 \\
80 & 0.385 & 1.2989 & -.3630 \\
90 & 0.000 & 1.3080 & -.3604 \\
100.00 & -.159 & 1.2989 & -.3600 \\
110.00 & -.302 & 1.2148 & -.3446 \\
120.00 & -.415 & 1.0684 & -.3166 \\
130.00 & -.486 & .8799 & -.2800 \\
140.00 & -.510 & .6743 & -.2394 \\
150.00 & -.494 & .4784 & -.2001 \\
160.00 & -.460 & .3172 & -.1685 \\
170.00 & -.230 & .2116 & -.5000 \\
180.00 & .000 & .1748 & .0000 \\
\hline & & &
\end{tabular}

\section{S809 CLN 189.dat}

S809 Airfoil, OSU data at $\mathrm{Re}=.75$ Million, Clean roughness

Adjusted for Post Stall at $\mathrm{r} / \mathrm{R}=.185^{* *}$

1 Number of airfoil tables in this file

.00 Table ID parameter

30.00 Stall angle (deg)**

.00 No longer used, enter zero

.00 No longer used, enter zero 


\begin{tabular}{|c|c|c|c|}
\hline \multirow{2}{*}{\multicolumn{4}{|c|}{$\begin{aligned} .00 & \text { No longer used, enter zero } \\
-.38 & \text { Zero lift angle of attack (deg) }\end{aligned}$}} \\
\hline & & & \\
\hline \multicolumn{2}{|c|}{7.12499} & \multicolumn{2}{|c|}{ Cn slope for zero lift (dimensionless) } \\
\hline \multicolumn{4}{|c|}{ 3.50 $\mathrm{Cn}$ at stall value for positive angle of attack** } \\
\hline-.8000 & & $\mathrm{Cn}$ at sta & 11 value for negative angle of attack \\
\hline 2.0000 & & Angle of & attack for minimum CD (deg) \\
\hline .0116 & & Minimu & $\mathrm{n}$ CD value \\
\hline-180.00 & 0.000 & .1748 & .0000 \\
\hline-170.00 & .230 & .2116 & .4000 \\
\hline-160.00 & 0.460 & .3172 & .1018 \\
\hline-150.00 & 0. .494 & .4784 & .1333 \\
\hline-140.00 & .510 & .6743 & 1727 \\
\hline-130.00 & 0. .486 & .8799 & .2132 \\
\hline-120.00 & 0. .415 & 1.0684 & 2498 \\
\hline-110.00 & 0. .302 & 1.2148 & .2779 \\
\hline-100.00 & 0. 159 & 1.2989 & 2933 \\
\hline-90.00 & .000 & 1.3080 & .2936 \\
\hline-80.00 & -.159 & 1.2989 & .2933 \\
\hline-70.00 & -.302 & 1.2148 & .2779 \\
\hline-60.00 & -.415 & 1.0684 & .2498 \\
\hline-50.00 & -.486 & .8799 & . 2132 \\
\hline-40.00 & -.510 & .6743 & .1727 \\
\hline-30.00 & -.494 & .4784 & .1333 \\
\hline-20.10 & -.560 & .3027 & .0612 \\
\hline-18.10 & -.670 & .3069 & .0904 \\
\hline-16.10 & -.790 & .1928 & .0293 \\
\hline-14.20 & -.840 & .0898 & -.0090 \\
\hline-12.20 & -.700 & .0553 & -.0045 \\
\hline-10.10 & -.630 & .0390 & -.0044 \\
\hline-8.20 & -.560 & .0233 & -.0051 \\
\hline-6.10 & -.640 & .0131 & .0018 \\
\hline-4.1 & -0.427 & 0.0134 & -.0216 \\
\hline-2.1 & -0.204 & 0.0119 & -.0282 \\
\hline 0.1 & 0.057 & 0.0122 & -.0346 \\
\hline 2 & 0.295 & 0.0116 & -.0405 \\
\hline 4.1 & 0.540 & 0.0144 & -.0455 \\
\hline 6.2 & 0.789 & 0.0146 & -.0507 \\
\hline 8.1 & 0.955 & 0.0162 & -.0404 \\
\hline 10.2 & 1.090 & 0.0274 & -.0321 \\
\hline 11.3 & 1.148 & 0.0303 & -.0281 \\
\hline 12.1 & 1.209 & 0.0369 & -.0284 \\
\hline 13.2 & 1.293 & 0.0509 & -.0322 \\
\hline 14.2 & 1.360 & 0.0648 & -.0361 \\
\hline 15.3 & 1.428 & 0.0776 & -.0363 \\
\hline 16.3 & 1.475 & 0.0917 & -.0393 \\
\hline 17.1 & 1.489 & 0.0994 & -.0398 \\
\hline 18.1 & 1.500 & 0.2306 & -.0983 \\
\hline 19.1 & 1.479 & 0.3142 & -.1242 \\
\hline 20.1 & 1.515 & 0.3186 & -.1155 \\
\hline 30 & 2.103 & 0.4784 & -.2459 \\
\hline 40 & 2.089 & 0.6743 & -.2813 \\
\hline 50 & 1.825 & 0.8799 & -.3134 \\
\hline 60 & 1.384 & 1.0684 & -.3388 \\
\hline 70 & 0.856 & 1.2148 & -.3557 \\
\hline 80 & 0.352 & 1.2989 & -.3630 \\
\hline 90 & 0.000 & 1.3080 & -.3604 \\
\hline
\end{tabular}




\begin{tabular}{llll}
100.00 & -.159 & 1.2989 & -.3600 \\
110.00 & -.302 & 1.2148 & -.3446 \\
120.00 & -.415 & 1.0684 & -.3166 \\
130.00 & -.486 & .8799 & -.2800 \\
140.00 & -.510 & .6743 & -.2394 \\
150.00 & -.494 & .4784 & -.2001 \\
160.00 & -.460 & .3172 & -.1685 \\
170.00 & -.230 & .2116 & -.5000 \\
180.00 & .000 & .1748 & .0000 \\
\hline
\end{tabular}

\section{S809_CLN_242.dat}

S809 Airfoil, OSU data at $\mathrm{Re}=.75$ Million, Clean roughness

Adjusted for Post Stall at $\mathrm{r} / \mathrm{R}=.242 * *$

1 Number of airfoil tables in this file

.00 Table ID parameter

17.10 Stall angle (deg)**

.00 No longer used, enter zero

.00 No longer used, enter zero

.00 No longer used, enter zero

-.38 Zero lift angle of attack (deg)

7.12499 Cn slope for zero lift (dimensionless)

$2.10 \mathrm{Cn}$ at stall value for positive angle of attack**

$-8000 \quad \mathrm{Cn}$ at stall value for negative angle of attack

$2.0000 \quad$ Angle of attack for minimum CD (deg)

$.0116 \quad$ Minimum $C D$ value

$\begin{array}{llll}-180.00 & .000 & .1748 \quad .0000\end{array}$

$\begin{array}{llll}-170.00 & .230 & .2116 \quad .4000\end{array}$

$\begin{array}{llll}-160.00 & .460 & .3172 & .1018\end{array}$

$\begin{array}{llll}-150.00 & .494 & .4784 & .1333\end{array}$

$\begin{array}{llll}-140.00 & .510 & .6743 & .1727\end{array}$

$\begin{array}{llll}-130.00 & .486 & .8799 & .2132\end{array}$

$\begin{array}{llll}-120.00 & .415 & 1.0684 & .2498\end{array}$

$\begin{array}{llll}-110.00 & .302 & 1.2148 & .2779\end{array}$

$\begin{array}{llll}-100.00 & .159 & 1.2989 & .2933\end{array}$

$\begin{array}{llll}-90.00 & .000 & 1.3080 & .2936\end{array}$

$\begin{array}{llll}-80.00 & -.159 & 1.2989 & .2933\end{array}$

$\begin{array}{llll}-70.00 & -.302 & 1.2148 & .2779\end{array}$

$\begin{array}{llll}-60.00 & -.415 & 1.0684 & .2498\end{array}$

$\begin{array}{llll}-50.00 & -.486 & .8799 & .2132\end{array}$

$\begin{array}{llll}-40.00 & -.510 & .6743 & .1727\end{array}$

$\begin{array}{llll}-30.00 & -.494 & .4784 & .1333\end{array}$

$\begin{array}{llll}-20.10 & -.560 & .3027 & .0612\end{array}$

$\begin{array}{llll}-18.10 & -.670 & .3069 & .0904\end{array}$

$\begin{array}{llll}-16.10 & -.790 & .1928 & .0293\end{array}$

$\begin{array}{lllll}-14.20 & -.840 & .0898 & -.0090\end{array}$

$\begin{array}{llll}-12.20 & -.700 & .0553 & -.0045\end{array}$

$\begin{array}{lllll}-10.10 & -.630 & .0390 & -.0044\end{array}$

$\begin{array}{llll}-8.20 & -.560 & .0233 & -.0051\end{array}$

$\begin{array}{llll}-6.10 & -.640 & .0131 & .0018\end{array}$

$\begin{array}{llll}-4.1 & -0.425 & 0.0134 & -.0216\end{array}$

$\begin{array}{llll}-2.1 & -0.205 & 0.0119 & -.0282\end{array}$

$\begin{array}{llll}0.1 & 0.055 & 0.0122 & -.0346\end{array}$

$\begin{array}{llll}2 & 0.296 & 0.0116 & -.0405\end{array}$

$\begin{array}{llll}4.1 & 0.540 & 0.0144 & -.0455\end{array}$

$\begin{array}{lllll}6.2 & 0.789 & 0.0146 & -.0507\end{array}$ 


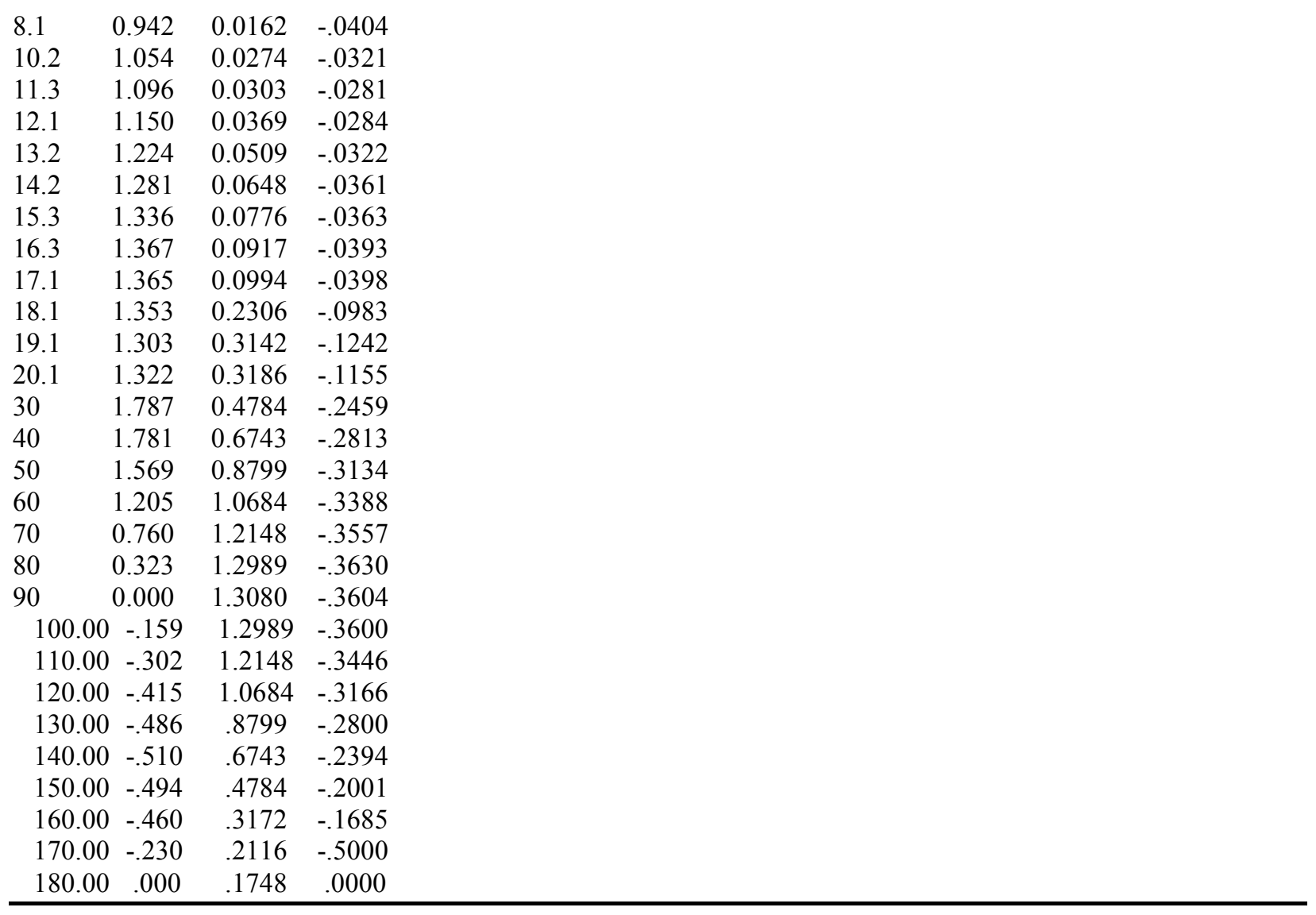

S809_CLN_298.dat

S809 Airfoil, OSU data at $\mathrm{Re}=.75$ Million, Clean roughness

Adjusted for Post Stall at $\mathrm{r} / \mathrm{R}=.298 * *$

1 Number of airfoil tables in this file

.00 Table ID parameter

16.30 Stall angle (deg)**

.00 No longer used, enter zero

.00 No longer used, enter zero

.00 No longer used, enter zero

-.38 Zero lift angle of attack (deg)

7.12499 Cn slope for zero lift (dimensionless)

$2.00 \mathrm{Cn}$ at stall value for positive angle of attack**

-.8000 Cn at stall value for negative angle of attack

$2.0000 \quad$ Angle of attack for minimum CD (deg)

.0116 Minimum CD value

$\begin{array}{llll}-180.00 & .000 & .1748 & .0000\end{array}$

$\begin{array}{llll}-170.00 & .230 & .2116 \quad .4000\end{array}$

$\begin{array}{llll}-160.00 & .460 & .3172 & .1018\end{array}$

$\begin{array}{llll}-150.00 & .494 & .4784 & .1333\end{array}$

$\begin{array}{llll}-140.00 & .510 & .6743 & .1727\end{array}$

$\begin{array}{llll}-130.00 & .486 & .8799 & .2132\end{array}$

$\begin{array}{llll}-120.00 & .415 & 1.0684 & .2498\end{array}$

$\begin{array}{llll}-110.00 & .302 & 1.2148 & .2779\end{array}$

$\begin{array}{llll}-100.00 & .159 & 1.2989 & .2933\end{array}$

$\begin{array}{llll}-90.00 & .000 & 1.3080 & .2936\end{array}$

$\begin{array}{llll}-80.00 & -.159 & 1.2989 & .2933\end{array}$ 


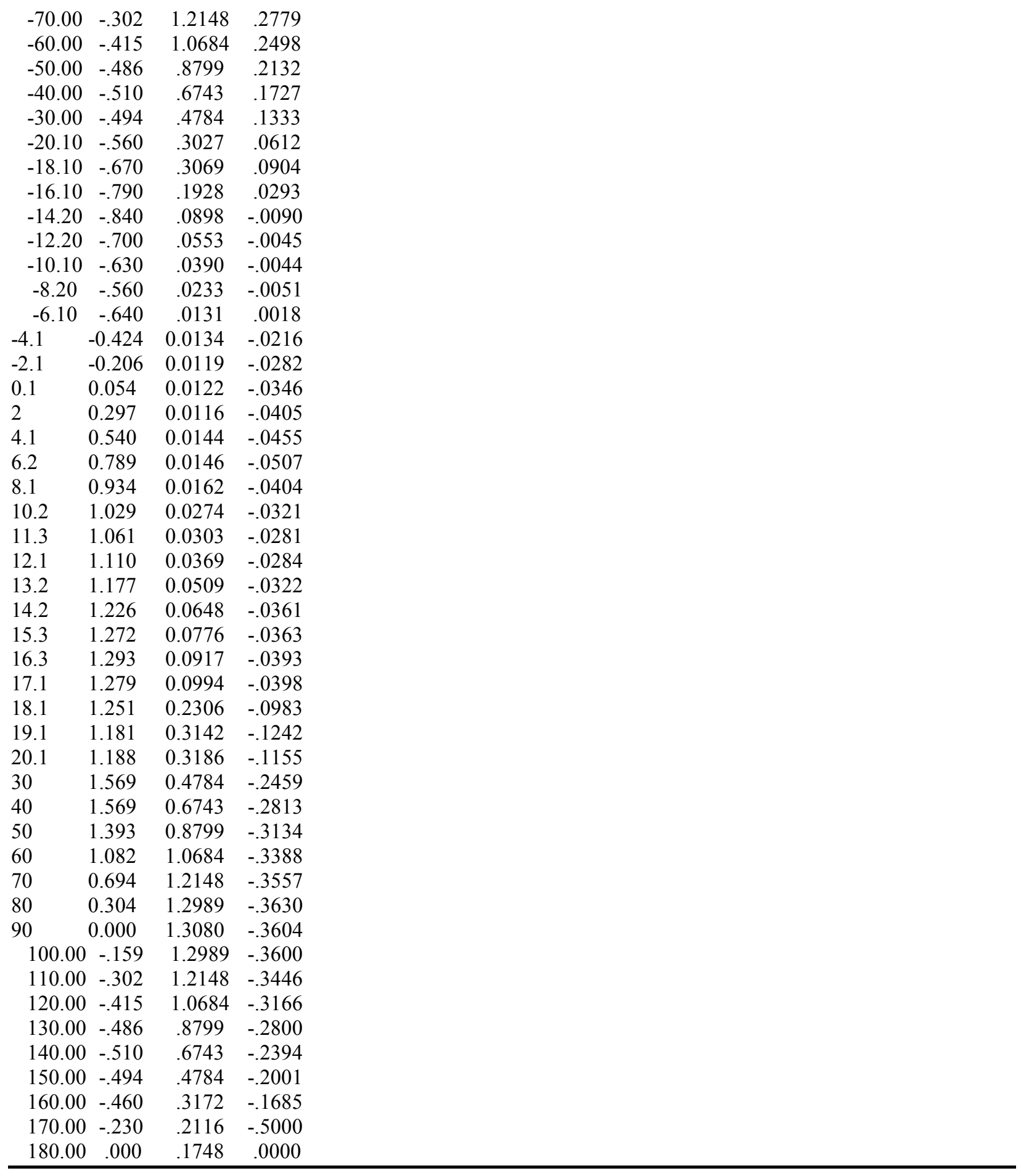

S809_CLN_354.dat

S809 Airfoil, OSU data at $\mathrm{Re}=.75$ Million, Clean roughness

Adjusted for Post Stall at $\mathrm{r} / \mathrm{R}=.354 * *$

1 Number of airfoil tables in this file

.00 Table ID parameter

16.30 Stall angle (deg)**

.00 No longer used, enter zero 


\begin{tabular}{|c|c|c|c|}
\hline .00 & \multicolumn{3}{|c|}{ No longer used, enter zero } \\
\hline .00 & \multicolumn{3}{|c|}{ No longer used, enter zero } \\
\hline \multirow{2}{*}{\multicolumn{3}{|c|}{$\begin{array}{l}-.38 \text { Zero lift angle of attack (deg) } \\
7.12499 \quad \text { Cn slope for zero lift (dimensionless) }\end{array}$}} & \\
\hline & & Cn slope & \\
\hline \multicolumn{4}{|c|}{$2.00 \mathrm{Cn}$ at stall value for positive angle of attack ${ }^{* *}$} \\
\hline \multicolumn{2}{|c|}{-.8000} & \multicolumn{2}{|c|}{$\mathrm{Cn}$ at stall value for negative angle of attack } \\
\hline \multicolumn{2}{|c|}{2.0000} & \multicolumn{2}{|c|}{ Angle of attack for minimum CD (deg) } \\
\hline \multicolumn{2}{|c|}{.0116} & \multicolumn{2}{|c|}{ Minimum $C D$ value } \\
\hline-180.00 & .000 & .1748 & .0000 \\
\hline-170.00 & .230 & .2116 & .4000 \\
\hline-160.00 & .460 & .3172 & .1018 \\
\hline-150.00 & .494 & .4784 & .1333 \\
\hline-140.00 & .510 & .6743 & .1727 \\
\hline-130.00 & .486 & .8799 & .2132 \\
\hline-120.00 & .415 & 1.0684 & .2498 \\
\hline-110.00 & .302 & 1.2148 & .2779 \\
\hline-100.00 & .159 & 1.2989 & .2933 \\
\hline-90.00 & .000 & 1.3080 & .2936 \\
\hline-80.00 & -.159 & 1.2989 & .2933 \\
\hline-70.00 & -.302 & 1.2148 & .2779 \\
\hline-60.00 & -.415 & 1.0684 & .2498 \\
\hline-50.00 & -.486 & .8799 & .2132 \\
\hline-40.00 & -.510 & .6743 & .1727 \\
\hline-30.00 & -.494 & .4784 & .1333 \\
\hline-20.10 & -.560 & .3027 & .0612 \\
\hline-18.10 & -.670 & .3069 & .0904 \\
\hline-16.10 & -.790 & .1928 & .0293 \\
\hline-14.20 & -.840 & .0898 & -.0090 \\
\hline-12.20 & -.700 & .0553 & -.0045 \\
\hline-10.10 & -.630 & .0390 & -.0044 \\
\hline-8.20 & -.560 & .0233 & -.0051 \\
\hline-6.10 & -.640 & .0131 & .0018 \\
\hline-4.1 & -0.423 & 0.0134 & -.0216 \\
\hline-2.1 & -0.207 & 0.0119 & -.0282 \\
\hline 0.1 & 0.053 & 0.0122 & -.0346 \\
\hline 2 & 0.298 & 0.0116 & -.0405 \\
\hline 4.1 & 0.540 & 0.0144 & -.0455 \\
\hline 6.2 & 0.790 & 0.0146 & -.0507 \\
\hline 8.1 & 0.927 & 0.0162 & -.0404 \\
\hline 10.2 & 1.008 & 0.0274 & -.0321 \\
\hline 11.3 & 1.032 & 0.0303 & -.0281 \\
\hline 12.1 & 1.077 & 0.0369 & -.0284 \\
\hline 13.2 & 1.138 & 0.0509 & -.0322 \\
\hline 14.2 & 1.182 & 0.0648 & -.0361 \\
\hline 15.3 & 1.220 & 0.0776 & -.0363 \\
\hline 16.3 & 1.233 & 0.0917 & -.0393 \\
\hline 17.1 & 1.209 & 0.0994 & -.0398 \\
\hline 18.1 & 1.168 & 0.2306 & -.0983 \\
\hline 19.1 & 1.082 & 0.3142 & -.1242 \\
\hline 20.1 & 1.079 & 0.3186 & -.1155 \\
\hline 30 & 1.390 & 0.4784 & -.2459 \\
\hline 40 & 1.395 & 0.6743 & -.2813 \\
\hline 50 & 1.248 & 0.8799 & -.3134 \\
\hline 60 & 0.981 & 1.0684 & -.3388 \\
\hline 70 & 0.640 & 1.2148 & -.3557 \\
\hline 80 & 0.288 & 1.2989 & -.3630 \\
\hline
\end{tabular}




\begin{tabular}{llll}
90 & 0.000 & 1.3080 & -.3604 \\
100.00 & -.159 & 1.2989 & -.3600 \\
110.00 & -.302 & 1.2148 & -.3446 \\
120.00 & -.415 & 1.0684 & -.3166 \\
130.00 & -.486 & .8799 & -.2800 \\
140.00 & -.510 & .6743 & -.2394 \\
150.00 & -.494 & .4784 & -.2001 \\
160.00 & -.460 & .3172 & -.1685 \\
170.00 & -.230 & .2116 & -.5000 \\
180.00 & .000 & .1748 & .0000 \\
\hline
\end{tabular}

\section{S809_CLN_410.dat}

S809 Airfoil, OSU data at $\mathrm{Re}=.75$ Million, Clean roughness

Adjusted for Post Stall at $\mathrm{r} / \mathrm{R}=.41 * *$

1 Number of airfoil tables in this file

.00 Table ID parameter

15.30 Stall angle (deg)**

.00 No longer used, enter zero

.00 No longer used, enter zero

.00 No longer used, enter zero

-.38 Zero lift angle of attack (deg)

7.12499 Cn slope for zero lift (dimensionless)

$1.90 \mathrm{Cn}$ at stall value for positive angle of attack**

$-.8000 \quad \mathrm{Cn}$ at stall value for negative angle of attack

$2.0000 \quad$ Angle of attack for minimum CD (deg)

$.0116 \quad$ Minimum $C D$ value

$\begin{array}{llll}-180.00 & .000 & .1748 & .0000\end{array}$

$\begin{array}{llll}-170.00 & .230 & .2116 & .4000\end{array}$

$\begin{array}{llll}-160.00 & .460 & .3172 & .1018\end{array}$

$\begin{array}{llll}-150.00 & .494 & .4784 & .1333\end{array}$

$\begin{array}{llll}-140.00 & .510 & .6743 & .1727\end{array}$

$\begin{array}{llll}-130.00 & .486 & .8799 & .2132\end{array}$

$\begin{array}{llll}-120.00 & .415 & 1.0684 & .2498\end{array}$

$\begin{array}{llll}-110.00 & .302 & 1.2148 & .2779\end{array}$

$\begin{array}{llll}-100.00 & .159 & 1.2989 & .2933\end{array}$

$\begin{array}{llll}-90.00 & .000 & 1.3080 & .2936\end{array}$

$\begin{array}{llll}-80.00 & -.159 & 1.2989 & .2933\end{array}$

$\begin{array}{llll}-70.00 & -.302 & 1.2148 & .2779\end{array}$

$\begin{array}{llll}-60.00 & -.415 & 1.0684 & .2498\end{array}$

$\begin{array}{llll}-50.00 & -.486 & .8799 & .2132\end{array}$

$\begin{array}{llll}-40.00 & -.510 & .6743 & .1727\end{array}$

$\begin{array}{llll}-30.00 & -.494 & .4784 & .1333\end{array}$

$\begin{array}{llll}-20.10 & -.560 & .3027 & .0612\end{array}$

$\begin{array}{llll}-18.10 & -.670 & .3069 & .0904\end{array}$

$\begin{array}{llll}-16.10 & -.790 & .1928 & .0293\end{array}$

$\begin{array}{llll}-14.20 & -.840 & .0898 & -.0090\end{array}$

$\begin{array}{llll}-12.20 & -.700 & .0553 & -.0045\end{array}$

$\begin{array}{llll}-10.10 & -.630 & .0390 & -.0044\end{array}$

$\begin{array}{llll}-8.20 & -.560 & .0233 & -.0051\end{array}$

$\begin{array}{llll}-6.10 & -.640 & .0131 & .0018\end{array}$

$\begin{array}{llll}-4.1 & -0.423 & 0.0134 & -.0216\end{array}$

$\begin{array}{lllll}-2.1 & -0.208 & 0.0119 & -.0282\end{array}$

$\begin{array}{llll}0.1 & 0.053 & 0.0122 & -.0346\end{array}$

$\begin{array}{lllll}2 & 0.298 & 0.0116 & -.0405\end{array}$

$\begin{array}{llll}4.1 & 0.540 & 0.0144 & -.0455\end{array}$ 


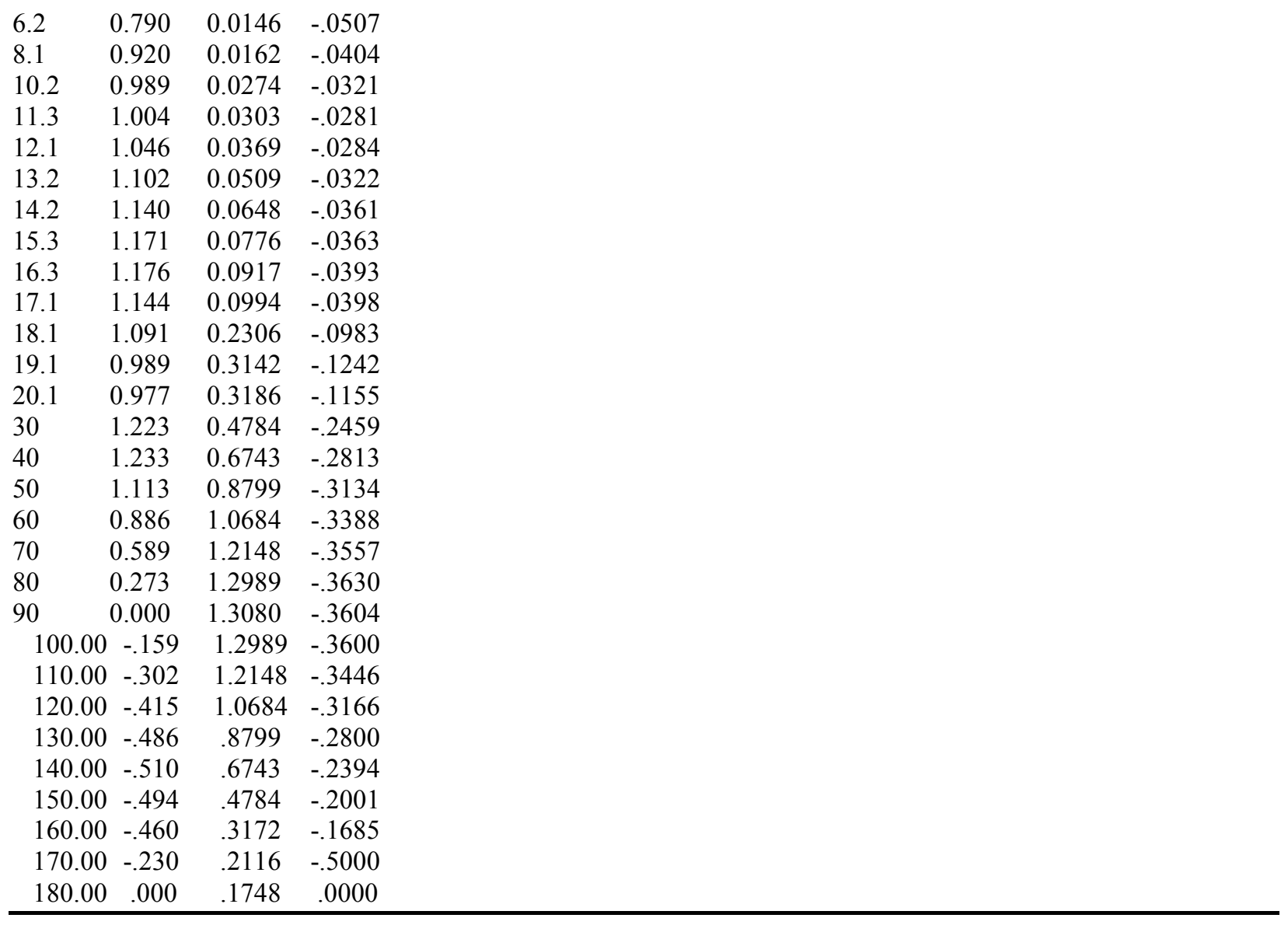

\section{S809_CLN_Outboard.dat}

S809 Airfoil, OSU data at $\mathrm{Re}=.75$ Million, Clean roughness

NREL/TP-442-7817 Appendix B, Viterna used aspect ratio=11

1 Number of airfoil tables in this file

.00 Table ID parameter

15.30 Stall angle (deg)

.00 No longer used, enter zero

.00 No longer used, enter zero

.00 No longer used, enter zero

-.38 Zero lift angle of attack (deg)

7.12499 Cn slope for zero lift (dimensionless)

$1.9408 \quad \mathrm{Cn}$ at stall value for positive angle of attack

-.8000 Cn at stall value for negative angle of attack

$2.0000 \quad$ Angle of attack for minimum CD (deg)

$.0116 \quad$ Minimum CD value

$\begin{array}{llll}-180.00 & .000 & .1748 \quad .0000\end{array}$

$\begin{array}{llll}-170.00 & .230 & .2116 & .4000\end{array}$

$\begin{array}{llll}-160.00 & .460 & .3172 & .1018\end{array}$

$\begin{array}{llll}-150.00 & .494 & .4784 & .1333\end{array}$

$\begin{array}{llll}-140.00 & .510 & .6743 & .1727\end{array}$

$\begin{array}{llll}-130.00 & .486 & .8799 & .2132\end{array}$

$\begin{array}{llll}-120.00 & .415 & 1.0684 & .2498\end{array}$

$\begin{array}{llll}-110.00 & .302 & 1.2148 & .2779\end{array}$

$\begin{array}{llll}-100.00 & .159 & 1.2989 & .2933\end{array}$

$\begin{array}{llll}-90.00 & .000 & 1.3080 & .2936\end{array}$ 


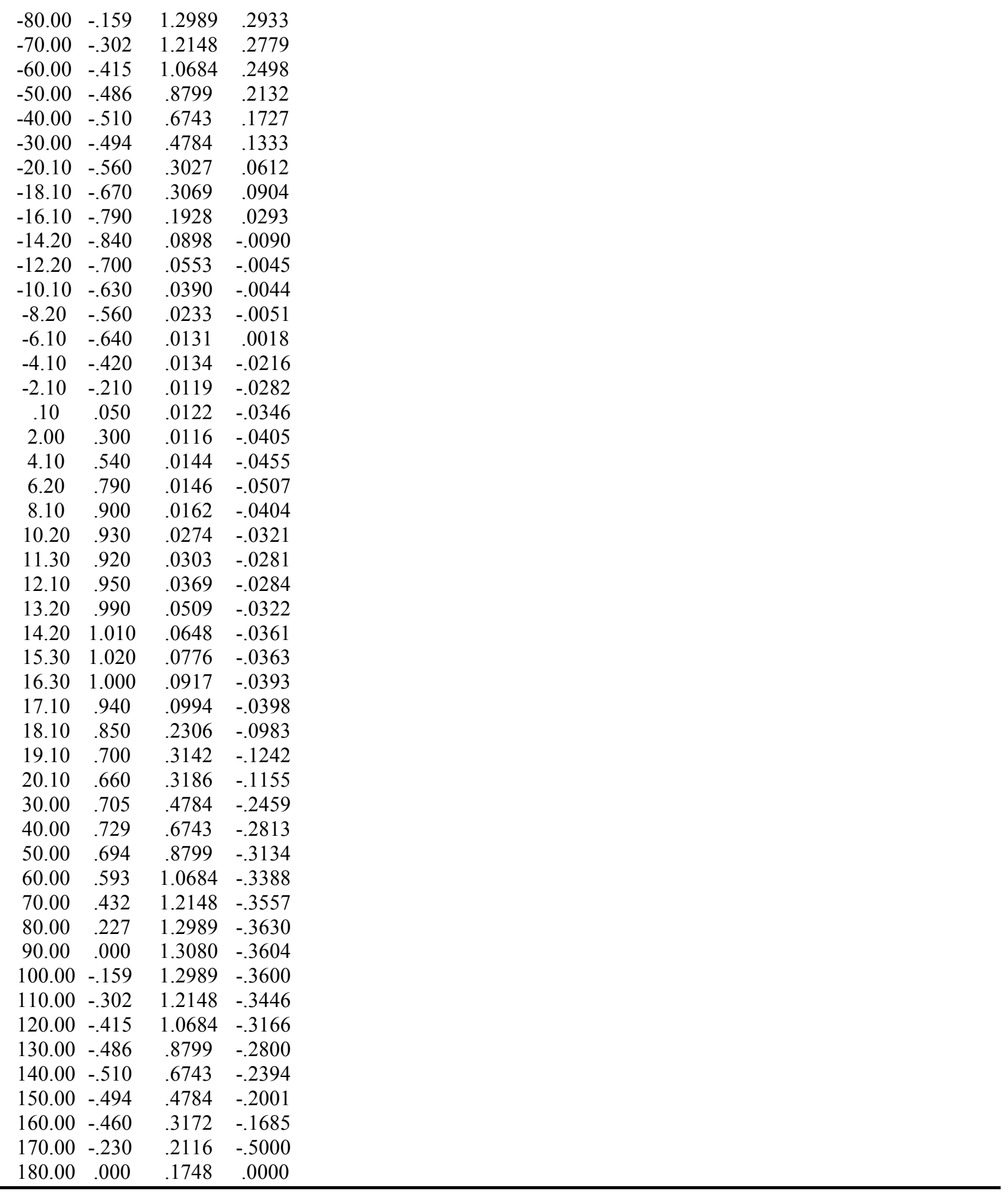




\section{Appendix V. Crunch Data File for Test Case $\mathbf{S 0 7 0 0 0 0 0}$}

S0700000.cru

Input file for analysis of .all files created using FAST_AD. The .all files are a concatenation of the .out and felement.plt data files. Compatible with Crunch Version 2.60.

$0 \quad$ Row number that contains channel titles; enter 0 if no titles are available or if titles are specified below

$0 \quad$ Row number that contains channel units; enter 0 if no units are available or if units are specified below

$5 \quad$ First row number of data

$0 \quad$ Number of data records to be read from file; enter 0 to automatically determine which rows to read

0.0,0.0 Start and end times of data records to be read; enter 0's to use all the data records in the file

False Output statistics?

False Output modified data?

True Create tab-delimited output?

"F11.4" Numerical-output format specifier; see manual for limitations

False Output aggregate analysis files? enter False for separate analysis files for each input file

"Aggregate" Root name of the aggregate files, if aggregates were specified above

$72 \quad$ Number of channels in each input file

$72 \quad$ Number of channels to use in analysis

Format for column info is: Col_Title(10 char max), Units(10 char max), Orig_Col_\#, Scale factor, Offset

"Time" $\quad$ "(sec)" $001 \quad 1.0 \quad 0.0$

"Yaw" "(deg)" $002 \quad 1.0 \quad 0.0$

"HH_WDir" "(deg)" $003 \quad 1.0 \quad 0.0$

"HH_WSpd" "(m/s)" $004 \quad 1.0 \quad 0.0$

"RtrĀzm" " "(deg)" $005 \quad 1.0 \quad 0.0$

"Bld1POut" $\quad$ "(cm)" $006 \quad 1.0 \quad 0.0$

"Bl1Pitch" "(deg)" $007 \quad 1.0 \quad 0.0$

"RotRPM" "(RPM)"008 $1.0 \quad 0.0$

"M1OuRt" $\quad$ "(kNm)" $009 \quad 1.0 \quad 0.0$

"M1InRt" " $\quad$ "(kNm)" $010 \quad 1.0 \quad 0.0$

"ShafTorq" "(kNm)" $011 \quad 1.0 \quad 0.0$

"BearYawM" "(kNm)" $012 \quad 1.0 \quad 0.0$

"Time" "(sec)" $013 \quad 1.0 \quad 0.0$

"VX21" "(m/s)" $014 \quad 1.0 \quad 0.0$

"VY21" $\quad$ "(m/s)" $015 \quad 1.0 \quad 0.0$

"VZ21" "(m/s)" $016 \quad 1.0 \quad 0.0$

$\begin{array}{llll}\text { "Alpha05" } \quad \text { "(deg)" } & 017 & 1.0 & 0.0\end{array}$

"CLift05" " (- ) $018 \quad 1.0 \quad 0.0$

"CDrag05" $\quad$ " ( - )" $019 \quad 1.0 \quad 0.0$

"AxInd05" $\quad$ "(- ) $020 \quad 1.0 \quad 0.0$

"TanInd05" $\quad$ "( - )" $021 \quad 1.0 \quad 0.0$

$\begin{array}{lllll}\text { "ForcN05" } \quad \text { "(N)" } & 022 & 1.0 & 0.0\end{array}$

$\begin{array}{llll}\text { "ForcT05" } \quad \text { "(N)" } & 023 & 1.0 & 0.0\end{array}$

"Alpha06" "(deg)" $024 \quad 1.0 \quad 0.0$

"CLift06" " ( - ) 0250.0 


\begin{tabular}{|c|c|c|c|c|}
\hline "CDrag06" & "( - ()" & 026 & 1.0 & 0.0 \\
\hline "AxInd06" & "( - ()" & 027 & 1.0 & 0.0 \\
\hline "TanInd06" & "( - ( )" & 028 & 1.0 & 0.0 \\
\hline "ForcN06" & "(N)" & 029 & 1.0 & 0.0 \\
\hline "ForcT06" & "(N)" & 030 & 1.0 & 0.0 \\
\hline "Alpha09" & "(deg)" & 031 & 1.0 & 0.0 \\
\hline "CLift09" & "( - ( ) & 032 & 1.0 & 0.0 \\
\hline "CDrag09" & "( - ( ) & 033 & 1.0 & 0.0 \\
\hline "AxInd09" & "( - ( )" & 034 & 1.0 & 0.0 \\
\hline "TanInd09" & "( - ( )" & 035 & 1.0 & 0.0 \\
\hline "ForcN09" & "(N)" & 036 & 1.0 & 0.0 \\
\hline "ForcT09" & "(N)" & 037 & 1.0 & 0.0 \\
\hline "Alpha10" & "(deg)" & 038 & 1.0 & 0.0 \\
\hline "CLift10" & "( - )" & 039 & 1.0 & 0.0 \\
\hline "CDrag10" & "( - ( )" & 040 & 1.0 & 0.0 \\
\hline "AxInd10" & ") - ( ) & 041 & 1.0 & 0.0 \\
\hline "TanInd10" & "( - ()" & 042 & 1.0 & 0.0 \\
\hline "ForcN10" & "(N)" & 043 & 1.0 & 0.0 \\
\hline "ForcT10" & "(N)" & 044 & 1.0 & 0.0 \\
\hline "Alpha13" & "(deg)" & 045 & 1.0 & 0.0 \\
\hline "CLift13" & "( - )" & 046 & 1.0 & 0.0 \\
\hline "CDrag13" & "( - ( )" & 047 & 1.0 & 0.0 \\
\hline "AxInd13" & "( - ()" & 048 & 1.0 & 0.0 \\
\hline "TanInd13" & "( - ( )" & 049 & 1.0 & 0.0 \\
\hline "ForcN13" & "(N)" & 050 & 1.0 & 0.0 \\
\hline "ForcT13" & "(N)" & 051 & 1.0 & 0.0 \\
\hline "Alpha16" & "(deg)" & 052 & 1.0 & 0.0 \\
\hline "CLift16" & ") - ( ) & 053 & 1.0 & 0.0 \\
\hline "CDrag16" & "( - ( )" & 054 & 1.0 & 0.0 \\
\hline "AxInd16" & "( - ( )" & 055 & 1.0 & 0.0 \\
\hline "TanInd16" & "( - ()" & 056 & 1.0 & 0.0 \\
\hline "ForcN16" & "(N)" & 057 & 1.0 & 0.0 \\
\hline "ForcT16" & "(N)" & 058 & 1.0 & 0.0 \\
\hline "Alpha17" & "(deg)" & 059 & 1.0 & 0.0 \\
\hline "CLift17" & ") - ( ) & 060 & 1.0 & 0.0 \\
\hline "CDrag17" & ") ( - ( ) & 061 & 1.0 & 0.0 \\
\hline "AxInd17" & "( - ( )" & 062 & 1.0 & 0.0 \\
\hline "TanInd17" & "( - ( )" & 063 & 1.0 & 0.0 \\
\hline "ForcN17" & "(N)" & 064 & 1.0 & 0.0 \\
\hline "ForcT17" & "(N)" & 065 & 1.0 & 0.0 \\
\hline "Alpha20" & "(deg)" & 066 & 1.0 & 0.0 \\
\hline "CLift20" & "( - ()" & 067 & 1.0 & 0.0 \\
\hline "CDrag20" & "( - ( ) & 068 & 1.0 & 0.0 \\
\hline "AxInd20" & ") - ( )" & 069 & 1.0 & 0.0 \\
\hline "TanInd20" & "( - ( )" & 070 & 1.0 & 0.0 \\
\hline "ForcN20" & "(N)" & 071 & 1.0 & 0.0 \\
\hline "ForcT20" & "(N)" & 072 & 1.0 & 0.0 \\
\hline
\end{tabular}

$0 \quad$ Number of output channels to be modified by the IIR filter; next 4 lines ignored if 0

$0 \quad$ Filter type: $1=$ LowPass, $2=$ HighPass, $3=$ BandPass

$0.0 \quad$ Low cutoff frequency $[\mathrm{Hz}]$; ignored for low-pass filters

$0.0 \quad$ High cutoff frequency $[\mathrm{Hz}]$; ignored for high-pass filters

91 Number of new, calculated channels to be generated; next line ignored if 0

Format for column info is: Col_Title(10 char max), Units(10 char max), Equation. Put each field in double quotes. 


\begin{tabular}{|c|c|c|c|}
\hline "YAW" & "[deg]" & "(C002-C003)" & 073 \\
\hline "VTUN" & "[m/s]" & "C004" & 074 \\
\hline "B3AZI" & "[deg]" & "C005" & 075 \\
\hline "B3FLAP" & "[deg]" & "((180.0/PI)*atan(C006/(100.0*5.029)))" & 076 \\
\hline "B3РITCH" & "[deg]" & "(C007 - 1.815)" & 077 \\
\hline "RPM" & "[RPM]' & "'C008" & 078 \\
\hline "B3RFP" & "[Nm]" & $"((\mathrm{C} 009 * \cos (\mathrm{C} 077 * \mathrm{PI} / 180.0)+\mathrm{C} 010 * \sin (\mathrm{C} 077 * \mathrm{PI} / 180.0)) * 1000.0) "$ & 079 \\
\hline "B3REP" & "[Nm]" & $"((-\mathrm{C} 009 * \sin (\mathrm{C} 077 * \mathrm{PI} / 180.0)+\mathrm{C} 010 * \cos (\mathrm{C} 077 * \mathrm{PI} / 180.0)) * 1000.0) "$ & 080 \\
\hline "LSSTQ" & "[Nm]" & "(C011*1000.0)" & 081 \\
\hline "NAYM" & "[Nm]" & "(-C012*1000.0)" & 082 \\
\hline "CN05" & "[ - ]" & "(C018* $\cos (\mathrm{C} 017 * \mathrm{PI} / 180.0)+\mathrm{C} 019 * \sin (\mathrm{C} 017 * \mathrm{PI} / 180.0)) "$ & 083 \\
\hline "CT05" & "[ - ]" & 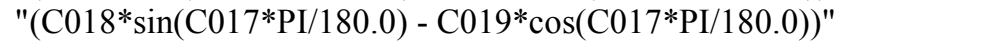 & 084 \\
\hline "N05" & "[N/m]" & $\begin{array}{l}\text { "((C022* } \cos ((4.815+17.89) * \mathrm{PI} / 180.0)+\mathrm{C} 023 * \sin ((4.815+ \\
17.89) * \mathrm{PI} / 180.0)) / 0.22985) "\end{array}$ & 085 \\
\hline "T05" & "[N/m]" & $\begin{array}{l}"((-\mathrm{C} 022 * \sin ((4.815+17.89) * \mathrm{PI} / 180.0)+\mathrm{C} 023 * \cos ((4.815+ \\
17.89) * \mathrm{PI} / 180.0)) / 0.22985) "\end{array}$ & 086 \\
\hline "QNORM05_N" & "[Pa]" & $"(\mathrm{C} 085 /(\mathrm{C} 083 * 0.727)) "$ & 087 \\
\hline "QNORM05-T" & "[Pa]" & "(C086/(C084*0.727))" & 088 \\
\hline "QNORM05" & "[Pa]" & "((C087+ C088)/2.0)" & 089 \\
\hline "CN06" & "[ - ]" & $"(\mathrm{C} 025 * \cos (\mathrm{C} 024 * \mathrm{PI} / 180.0)+\mathrm{C} 026 * \sin (\mathrm{C} 024 * \mathrm{PI} / 180.0)) "$ & 090 \\
\hline "СТ06" & "[ - ]" & $"(\mathrm{C} 025 * \sin (\mathrm{C} 024 * \mathrm{PI} / 180.0)-\mathrm{C} 026 * \cos (\mathrm{C} 024 * \mathrm{PI} / 180.0)) "$ & 091 \\
\hline "N06" & "[N/m]" & $\begin{array}{l}"((\mathrm{C} 029 * \cos ((4.815+13.33) * \mathrm{PI} / 180.0)+\mathrm{C} 030 * \sin ((4.815+ \\
13.33) * \mathrm{PI} / 180.0)) / 0.22985) "\end{array}$ & 092 \\
\hline "T06" & "[N/m]" & $\begin{array}{l}\text { "((-C029* } \sin ((4.815+13.33) * \mathrm{PI} / 180.0)+\mathrm{C} 030 * \cos ((4.815+ \\
13.33) * \mathrm{PI} / 180.0)) / 0.22985) "\end{array}$ & 093 \\
\hline "QNORM06_N" & "[Pa]" & "(C092/(C090*0.704))" & 094 \\
\hline "QNORM06_T" & "[Pa]" & "(C093/(C091*0.704))" & 095 \\
\hline "QNORM06" & "[Pa]" & "((C094 + C095)/2.0)" & 096 \\
\hline "CN30" & "[ - ]" & $"((1.0-0.68431) * \mathrm{C} 083+0.68431 * \mathrm{C} 090) "$ & 097 \\
\hline "СТ30" & "[ - ]" & $"((1.0-0.68431) * \mathrm{C} 084+0.68431 * \mathrm{C} 091) "$ & 098 \\
\hline "N30" & "[N/m]" & "((1.0 - 0.68431)*C $085+0.68431 * \mathrm{C} 092) "$ & 099 \\
\hline "Т30" & "[N/m]" & $"((1.0-0.68431) * \mathrm{C} 086+0.68431 * \mathrm{C} 093) "$ & 100 \\
\hline "QNORM30" & "[Pa]" & $"((1.0-0.68431) * \mathrm{C} 089+0.68431 * \mathrm{C} 096) "$ & 101 \\
\hline "CM30" & "[ - ]" & "0" & 102 \\
\hline "M30" & "[N]" & "0" & 103 \\
\hline "CN09" & "[ - ]" & $"(\mathrm{C} 032 * \cos (\mathrm{C} 031 * \mathrm{PI} / 180.0)+\mathrm{C} 033 * \sin (\mathrm{C} 031 * \mathrm{PI} / 180.0)) "$ & 104 \\
\hline "СТ09" & "[ - ]" & 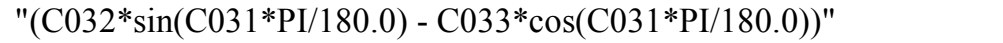 & 105 \\
\hline "N09" & "[N/m]" & $\begin{array}{l}"((\mathrm{C} 036 * \cos ((4.815+5.52) * \mathrm{PI} / 180.0)+\mathrm{C} 037 * \sin ((4.815+ \\
\left.\left.\left.5.52)^{*} \mathrm{PI} / 180.0\right)\right) / 0.22985\right) "\end{array}$ & 106 \\
\hline "T09" & "[N/m]" & $\begin{array}{l}"((-\mathrm{C} 036 * \sin ((4.815+5.52) * \mathrm{PI} / 180.0)+\mathrm{C} 037 * \cos ((4.815+ \\
5.52) * \mathrm{PI} / 180.0)) / 0.22985) "\end{array}$ & 107 \\
\hline "QNORM09_N" & "[Pa]" & "(C106/(C104*0.634))" & 108 \\
\hline "QNORM09_T" & "[Pa]" & "(C107/(C105*0.634))" & 109 \\
\hline "QNORM09" & "[Pa]" & "((C108 + C109)/2.0)" & 110 \\
\hline "CN10" & "[ - ]" & $"(\mathrm{C} 039 * \cos (\mathrm{C} 038 * \mathrm{PI} / 180.0)+\mathrm{C} 040 * \sin (\mathrm{C} 038 * \mathrm{PI} / 180.0)) "$ & 111 \\
\hline "CT10" & "[ - ]" & 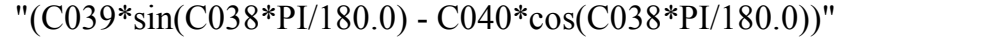 & 112 \\
\hline "N10" & "[N/m]" & $\begin{array}{l}\text { "((C043* } \cos ((4.815+3.81) * \mathrm{PI} / 180.0)+\mathrm{C} 044 * \sin ((4.815+ \\
\left.\left.\left.3.81)^{*} \mathrm{PI} / 180.0\right)\right) / 0.22985\right) "\end{array}$ & 113 \\
\hline "T10" & "[N/m]" & $\begin{array}{l}\text { "((-C043* } \sin ((4.815+3.81) * \mathrm{PI} / 180.0)+\mathrm{C} 044 * \cos ((4.815+ \\
3.81) * \mathrm{PI} / 180.0)) / 0.22985) "\end{array}$ & 114 \\
\hline "QNORM10_N" & "[Pa]" & "(C113/(C111*0.611))" & 115 \\
\hline "QNORM10_T" & "[Pa]" & "(C114/(C112*0.611))" & 116 \\
\hline "QNORM10" & "[Pa]" & "((C115 + C116)/2.0)" & 117 \\
\hline "CN47" & "[ - ]" & $"((1.0-0.33093) * \mathrm{C} 104+0.33093 * \mathrm{C} 111) "$ & 118 \\
\hline "CT47" & "[ - ]" & $"((1.0-0.33093) * \mathrm{C} 105+0.33093 * \mathrm{C} 112) "$ & 119 \\
\hline "N47" & "[N/m]" & "((1.0 - 0.33093)*C106 + 0.33093*C113)" & 120 \\
\hline
\end{tabular}




\begin{tabular}{|c|c|c|c|}
\hline "T47" & "[N/m]" & $"((1.0-0.33093) * \mathrm{C} 107+0.33093 * \mathrm{C} 114) "$ & 121 \\
\hline "QNORM47" & "[Pa]" & $"((1.0-0.33093) * \mathrm{C} 110+0.33093 * \mathrm{C} 117) "$ & 122 \\
\hline "CM47" & "[ - ]" & "0" & 123 \\
\hline "M47" & "[N]" & "0" & 124 \\
\hline "CN63" & "[ - ]" & "(C046* $\cos (\mathrm{C} 045 * \mathrm{PI} / 180.0)+\mathrm{C} 047 * \sin (\mathrm{C} 045 * \mathrm{PI} / 180.0)) "$ & 125 \\
\hline "CT63" & "[ - ]" & 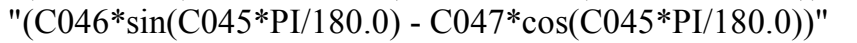 & 126 \\
\hline "N63" & "[N/m]" & $\begin{array}{l}\text { "((C050* } \cos ((4.815+1.21) * \mathrm{PI} / 180.0)+\mathrm{C} 051 * \sin ((4.815+ \\
\left.\left.\left.1.21)^{*} \mathrm{PI} / 180.0\right)\right) / 0.22985\right) "\end{array}$ & 127 \\
\hline "T63" & "[N/m]" & $\begin{array}{l}\text { "((-C050* } \sin ((4.815+1.21) * \mathrm{PI} / 180.0)+\mathrm{C} 051 * \cos ((4.815+ \\
\left.\left.\left.1.21)^{*} \mathrm{PI} / 180.0\right)\right) / 0.22985\right) "\end{array}$ & 128 \\
\hline "QNORM63_N" & "[Pa]" & "(C127/(C125*0.542))" & 129 \\
\hline "QNORM63_T" & "[Pa]" & "(C128/(C126*0.542))" & 130 \\
\hline "QNORM63" & "[Pa]" & "((C129+ C130)/2.0)" & 131 \\
\hline "CM63" & "[ - ]" & "0" & 132 \\
\hline "M63" & "[N]" & "0" & 133 \\
\hline "CN16" & "[ - ]" & "(C053* $\cos (\mathrm{C} 052 * \mathrm{PI} / 180.0)+\mathrm{C} 054 * \sin (\mathrm{C} 052 * \mathrm{PI} / 180.0)) "$ & 134 \\
\hline "CT16" & "[ - ]" & 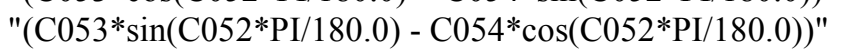 & 135 \\
\hline "N16" & "[N/m]" & $\begin{array}{l}\text { "((C057* } \cos ((4.815-0.15) * \mathrm{PI} / 180.0)+\mathrm{C} 058 * \sin ((4.815- \\
0.15) * \mathrm{PI} / 180.0)) / 0.22985) "\end{array}$ & 136 \\
\hline "T16" & "[N/m]" & $\begin{array}{l}\text { "((-C057* } \sin ((4.815-0.15) * \mathrm{PI} / 180.0)+\mathrm{C} 058 * \cos ((4.815- \\
0.15) * \mathrm{PI} / 180.0)) / 0.22985) "\end{array}$ & 137 \\
\hline "QNORM16 N" & "[Pa]" & "(C136/(C134*0.472))" & 138 \\
\hline "QNORM16-"T" & "[Pa]" & "(C137/(C135*0.472))" & 139 \\
\hline "QNORM16" & "[Pa]" & "((C138 + C139)/2.0)" & 140 \\
\hline "CN17" & "[ - ]" & "(C060* $\cos (\mathrm{C} 059 * \mathrm{PI} / 180.0)+\mathrm{C} 061 * \sin (\mathrm{C} 059 * \mathrm{PI} / 180.0)) "$ & 141 \\
\hline "CT17" & "[ - ]" & 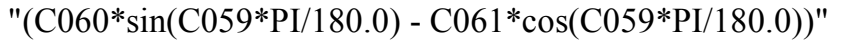 & 142 \\
\hline "N17" & "[N/m]" & 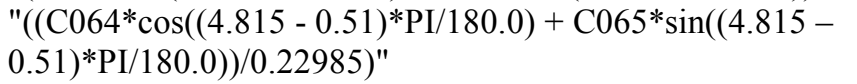 & 143 \\
\hline "T17" & "[N/m]" & $\begin{array}{l}\text { "((-C064* } \sin ((4.815-0.51) * \mathrm{PI} / 180.0)+\mathrm{C} 065 * \cos ((4.815- \\
0.51) * \mathrm{PI} / 180.0)) / 0.22985) "\end{array}$ & 144 \\
\hline "QNORM17 N" & "[Pa]" & "(C143/(C141*0.448))" & 145 \\
\hline "QNORM17-T" & "[Pa]" & "(C144/(C142*0.448))" & 146 \\
\hline "QNORM17" & "[Pa]" & "((C145+C146)/2.0)" & 147 \\
\hline "CN80" & "[ - ]" & $"((1.0-0.62407) * \mathrm{C} 134+0.62407 * \mathrm{C} 141) "$ & 148 \\
\hline "СТ80" & "[ - ]" & $"((1.0-0.62407) * \mathrm{C} 135+0.62407 * \mathrm{C} 142) "$ & 149 \\
\hline "N80" & "[N/m]" & $"((1.0-0.62407) * \mathrm{C} 136+0.62407 * \mathrm{C} 143) "$ & 150 \\
\hline "T80" & "[N/m]" & $"((1.0-0.62407) * \mathrm{C} 137+0.62407 * \mathrm{C} 144) "$ & 151 \\
\hline "QNORM80" & "[Pa]" & $"((1.0-0.62407) * \mathrm{C} 140+0.62407 * \mathrm{C} 147) "$ & 152 \\
\hline "CM80" & "[ - ]" & "0" & 153 \\
\hline "M80" & "[N]" & "0" & 154 \\
\hline "CN95" & "[ - ]" & "(C067* $\left.\cos (\mathrm{C} 066 * \mathrm{PI} / 180.0)+\mathrm{C} 068 * \sin \left(\mathrm{C} 066^{*} \mathrm{PI} / 180.0\right)\right) "$ & 155 \\
\hline "СТ95" & "[ - ]" & "(C067* $\sin (\mathrm{C} 066 * \mathrm{PI} / 180.0)-\mathrm{C} 068 * \cos (\mathrm{C} 066 * \mathrm{PI} / 180.0)) "$ & 156 \\
\hline "N95" & "[N/m]" & $\begin{array}{l}"((\mathrm{C} 071 * \cos ((4.815-1.49) * \mathrm{PI} / 180.0)+\mathrm{C} 072 * \sin ((4.815- \\
1.49) * \mathrm{PI} / 180.0)) / 0.22985) "\end{array}$ & 157 \\
\hline "T95" & "[N/m]" & $\begin{array}{l}\text { "((-C071* } \sin ((4.815-1.49) * \mathrm{PI} / 180.0)+\mathrm{C} 072 * \cos ((4.815- \\
1.49) * \mathrm{PI} / 180.0)) / 0.22985) "\end{array}$ & 158 \\
\hline "QNORM95_N" & "[Pa]" & "(C157/(C155*0.378))" & 159 \\
\hline "QNORM95_T" & "[Pa]" & "(C158/(C156*0.378))" & 160 \\
\hline "QNORM95" & "[Pa]" & "((C159+ C160)/2.0)" & 161 \\
\hline "CM95" & "[ - ]" & "0" & 162 \\
\hline "M95" & "[N]" & "0" & 163 \\
\hline
\end{tabular}

$0 \quad$ Number of channels to have moving averages generated for them; next line ignored if 0 Format for moving-average info is: Col_Title(10 char max), channel \#, averaging period

001 Channel number of time 
$0 \quad$ Number of channel pair(s) to have load roses generated for them; next line ignored if 0

Format for column info is: Rose_Title( 8 char max), 0 degree load, 90 degree load, \# sectors

45 Number of channels to be azimuth averaged; next four lines ignored if 0

$073,074,075,076,077,078,079,080,081,082,101,122,131,152,161,097,098,102,118,119,123,125,126,132,148,149,15$

$3,155,156,162,099,100,103,120,121,124,127,128,133,150,151,154,157,158,163$

$360 \quad$ Number of azimuth bins $=$ number of equally spaced sectors

005 Channel number of azimuth angle

True Output azimuth averages to a file?

$0 \quad$ Number of channel pairs that will have their crosstalk removed; next line ignored if 0

Format for crosstalk info is: OutCol \#1, OutCol \#2, XT(1,1), XT(1,2), XT(2,1), XT(2,2).

$0 \quad$ Number of channels to be modified by the peak finder; next line ignored if 0

0

$0 \quad$ Number of channels that will have probability density functions (PDFs) generated for them; next 2 lines ignored if 0

$20 \quad$ Number of bins used

Format for column info is: Column \#, Minimum, Maximum. If Min=Max=0, autocalculate them.

$0 \quad$ Number of channels that will have rainflow cycle counts generated for them; next 6 lines ignored if 0

1 Rainflow counting period [sec]

False Normalize rainflow cycle counts by bin width?

True For bins with zero counts, output a space if we are using tab-delimited output?

10 Number of rainflow range bins; enter 0 to output the actual cycles instead of binned cycles

$1 \quad$ Number of rainflow means bins; enter 1 to output ranges only

Format for column info is: Column \#, Max Range, Min Mean, Max Mean.

$0 \quad$ Number of groups of parameters that will have their extreme events recorded; next line ignored if 0

Format for column info is: Group_Title(100 char max), \#ExtCols, ColList(\#ExtCols long), \#InfCols(may be 0), ColList(\#InfCols long)

$0 \quad$ Number of channels will have statistics put in separate summary files; next line ignored if 0

0

$0 \quad$ Number of channels that will have their statistics extrapolated; next line ignored if 0

Format for statistics info is: Col_\#, Hours_to_extrapolate_to, Quantile desired

$1 \quad$ Number of input files to read

S0700000.all 


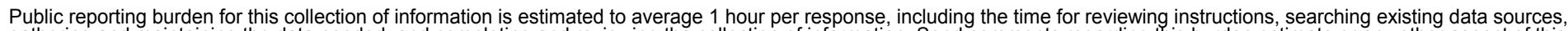

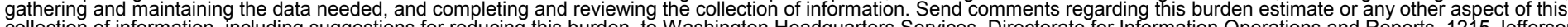
ervices, Directorate for Information Operations and Reports, 1215 Jefferson Davis Highway, Suite 1204, Arlington, VA 22202-4302, and to the Office of Management and Budget, Paperwork Reduction Project (0704-0188), Washington, DC 20503.

\begin{tabular}{|l|l|l} 
1. AGENCY USE ONLY (Leave blank) & $\begin{array}{c}\text { 2. REPORT DATE } \\
\text { December 2003 }\end{array}$ & $\begin{array}{c}\text { 3. REPORT TYPE AND DATES COVERED } \\
\text { Technical Report }\end{array}$ \\
\hline
\end{tabular}

\section{TITLE AND SUBTITLE}

Modeling of the UAE Wind Turbine for Refinement of FAST_AD

6. $\operatorname{AUTHOR}(\mathrm{S})$

J.M. Jonkman

7. PERFORMING ORGANIZATION NAME(S) AND ADDRESS(ES)

National Renewable Energy Laboratory

1617 Cole Blvd.

Golden, CO 80401-3393

5. FUNDING NUMBERS

WER3.2010

9. SPONSORING/MONITORING AGENCY NAME(S) AND ADDRESS(ES

10. SPONSORING/MONITORING AGENCY REPORT NUMBER

11. SUPPLEMENTARY NOTES

12a. DISTRIBUTION/AVAILABILITY STATEMENT

National Technical Information Service

U.S. Department of Commerce

5285 Port Royal Road

Springfield, VA 22161
8. PERFORMING ORGANIZATION REPORT NUMBER

NREL/TP-500-34755

13. ABSTRACT (Maximum 200 words)

The Unsteady Aerodynamics Experiment (UAE) research wind turbine was modeled both aerodynamically and structurally in the FAST_AD wind turbine design code, and its response to wind inflows was simulated for a sample of test cases. A study was condūcted to determine why wind turbine load magnitude discrepancies-inconsistencies in aerodynamic force coefficients, rotor shaft torque, and out-of-plane bending moments at the blade root across a range of operating conditionsexist between load predictions made by FAST_AD and other modeling tools and measured loads taken from the actual UAE wind turbine during the NASA-Ames wind tunnel tests. The acquired experimental test data represent the finest, most accurate set of wind turbine aerodynamic and induced flow field data available today. A sample of the FAST_AD model input parameters most critical to the aerodynamics computations was also systematically perturbed to determine their effect on load and performance predictions. Attention was focused on the simpler upwind rotor configuration, zero yaw error test cases. Inconsistencies in input file parameters, such as aerodynamic performance characteristics, explain a noteworthy fraction of the load prediction discrepancies of the various modeling tools.

14. SUBJECT TERMS

wind energy; wind turbine; FAST_AD; modeling tools; design codes; UAE; unsteady aerodynamics experiment

16. PRICE CODE

17. SECURITY CLASSIFICATION OF REPORT

Unclassified
18. SECURITY CLASSIFICATION OF THIS PAGE Unclassified
19. SECURITY CLASSIFICATION OF ABSTRACT

Unclassified
20. LIMITATION OF ABSTRACT

UL 